\title{
"Was that my misunderstanding?" Managing miscommunication and problematic talk at work
}

\author{
by
}

\section{Maria Stubbe}

\author{
A thesis \\ submitted to Victoria University of Wellington \\ in fulfilment of the requirements for the degree of \\ Doctor of Philosophy in Linguistics
}

Victoria University of Wellington March 2010 



\section{Table of contents}

Page

Acknowledgements vi

Abstract vii

\section{Introduction}

The problem with workplace communication $\quad \mathrm{p} 1$

1.1 Rationale for the study $\quad \mathrm{p} 1$

1.2 Background p 3

1.3 Objectives and scope $\quad$ p 9

2 Literature review (a)

Theoretical perspectives on workplace discourse p 13

2.1 Introduction $\quad \mathrm{p} 13$

2.2 Theoretical frameworks $\quad$ p 14

2.2.1 Epistemological positions $\quad \mathrm{p} 15$

2.2.2 Definitions of discourse p 17

2.3 Methodologies p 19

2.3.1 Conversation Analysis $\quad$ p 19

2.3.2 Pragmatics and politeness theory p 21

2.3.3 Sociolinguistics p 23

2.3.4 Social psychology p 25

2.3.5 Critical discourse theory p 26

2.3.6 Organisational theory p 29

2.4 Summary and discussion p 34 
$3 \quad$ Literature review (b)

Miscommunication and problematic talk p 37

3.1 Introduction $\quad$ p 37

3.2 Existing models of miscommunicative talk p 38

3.2.1 Some problems of definition $\quad \mathrm{p} 38$

3.2.2 An "integrative" model? p 44

3.3 Miscommunication in the workplace p 53

3.3.1 Interpersonal and intergroup miscommunication $\quad \mathrm{p} 55$

3.3.2 Competing and conflicting discourses $\quad$ p 61

3.3.3 Miscommunication in complex systems p 64

3.4 Summary and discussion $\quad$ p 68

3.5 Research Questions $\quad$ p 73

\section{Methodology}

Analytic approach and research design $\quad \mathrm{p} 75$

4.1 Introduction $\quad \mathrm{p} 75$

4.2 Analytic approach $\quad$ p 76

4.2.1 Methodological framework $\quad \mathrm{p} 76$

4.2.2 Analytic focus and tools $\quad \mathrm{p} 78$

4.3 The original LWP data collection methodology p 81

4.3.1 Designing the methodology p 81

4.3.2 Basic components of the participatory model p 83

4.3.3 Original data collection and projects $\quad \mathrm{p} 85$

4.3.4 Strengths and limitations $\quad$ p 86

4.4 Action research with a factory team: the adapted methodology $\mathrm{p} 89$

4.4.1 Developing the case study methodology p 90

4.4.2 Data collection p 91

4.5 The data: from office to production line $\quad$ p 94 
5 Developing a working model

Page

What exactly is miscommunication anyway? p 97

5.1 Introduction $\quad$ p 97

5.2 Analytic framework p 101

5.2.1 Issues of practical relevance p 101

5.2.2 Analytic process and dimensions p 106

5.3 Illustrative data analysis $\quad \mathrm{p} 118$

5.3.1 Case 1: 'Was that my misunderstanding?' p 119

5.3.2 Case 2: 'A subject dear to our hearts' p 125

5.3.3 Case 3: 'Power plays' p 130

5.4 Refining the analytic approach p 136

6 (Not) 'getting the message across'?

Problematic talk on the factory floor p 139

6.1 Introduction p 139

6.2 Ethnographic overview: the Power Rangers p 140

6.2.1 The team and its working environment p 140

6.2.2 Communication challenges p 143

6.2.3 Sources of miscommunication p 145

6.3 The Power Rangers team: a discursive community of practice p 148

6.3.1 Engaging in tasks p 152

6.3.2 Maintaining relationships p 155

6.3.3 Displaying group membership p 157

$\begin{array}{ll}6.4 \text { Conclusion } & \text { p } 167\end{array}$ 
7 Managing the contradictions

Page

The discursive strategies of the team leader p 169

$\begin{array}{ll}7.1 \text { Introduction } & \text { p } 169\end{array}$

7.2 Ethnographic overview: the TCO p 171

7.2.1 Personal profile and role p 171

7.2.2 Management style and strategies $\quad \mathrm{p} 172$

7.3 Discursive management of a problematic incident p 174

7.4 Applying a critical lens $\quad$ p 181

7.4.1 Power, identity and miscommunication p 181

7.4.2 Managing the contradictions $\quad \mathrm{p} 185$

7.5 Conclusion p 203

$8 \quad$ Problem or paradox?

The elephant in the room p 205

8.1 Introduction p 205

8.2 Summary of key findings p 208

8.3 Implications and applications $\quad$ p 211

8.4 Concluding remarks p 214

$\begin{array}{ll}\text { Appendix } & \mathrm{p} 217\end{array}$

1 Transcription conventions $\quad$ p 217

2 Research documentation p 218

References $\quad$ p 221 


\section{Tables and Figures}

Page

Table 1.1 Research objectives p 10

$\begin{array}{lll}\text { Table 2.1 } & \text { Research questions } 73\end{array}$

Figure 4.1 The action research cycle p 84

Table 4.1 LWP Data Sets: Number of recorded interactions $\quad$ p 94

Table 5.1 Analytic framework p 109

Table 5.2: $\quad$ Case 2 interaction structure p 126

$\begin{array}{lll}\text { Table 6.1 Recurring issues } & \text { p } 147\end{array}$

Table 7.1 Types of power relationship p 184

Table 7.2 Identity positions constructed by the TCO p 187 


\section{Acknowledgments}

I gratefully acknowledge the following people and organisations for their invaluable support and assistance with this doctoral project:

Most especially, the members of the 'Power Rangers' team, the management of Unilever Australasia, and the many other individuals and organisations who participated in the Language in the Workplace Project between 1996 and 2003, for their generosity in contributing their time, their data and their insights to this study. This project would not have been possible without their active and enthusiastic participation in the research.

My supervisors Derek Wallace and Paul Nation. The completion of this thesis owes much to their practical wisdom and unfailing quiet reassurance at several critical junctures that it remained both a possible and a worthwhile project.

My previous colleagues and associates from the Language in the Workplace Project team, including especially Janet Holmes, Bernadette Vine, Deborah Jones, Pascal Brown, Chris Lane, Megan Ingle, Tina Chiles, George Major, Louise Burns, Meredith Marra, Nicola Daly and all the many research assistants who have contributed over the years. This thesis project grew out of this wider programme of research into workplace communication and as such has built on the combined efforts and teamwork of many others.

Fellow members and associates of my current research team, the Applied Research on Communication in Health (ARCH) Group, in particular Tony Dowell, Lindsay Macdonald, Kevin Dew and Rachel Tester, as well as my other colleagues in the Department of Primary Health Care and General Practice at the University of Otago, Wellington. The thesis has benefitted greatly from the free exchange and crossfertilisation of ideas in this multidisciplinary setting, and the unstinting practical and moral support provided to me in the closing stages of the research and writing was particularly helpful.

The School of Linguistics and Applied Language Studies, Victoria University of Wellington and the Wellington School of Medicine and Health Sciences, Otago University for between them providing my academic base and resources throughout the course of this project.

The Foundation for Research, Science and Technology and Victoria University of Wellington for funding support between 1996 and 2003.

The Victoria University of Wellington Alumni Association for the award of a Postgraduate Scholarship in 2003.

Finally, my deepest thanks must go to my wonderful family and friends who have so patiently tolerated my preoccupation with this work over many years, and most especially during the final six months. I could not have completed this thesis without your continued and generous encouragement and support. 


\section{Abstract}

Where workplace communication is ineffective or problematic there will often be negative outcomes for individuals, teams or organisations as a whole. This thesis examines these issues using data from a variety of New Zealand workplaces, including most importantly an in depth case study of problematic communication in a multicultural factory team. The thesis provides an illustrative analysis of the communication issues that typically arise in these workplaces and the discursive strategies used to manage miscommunication and problematic talk, as well as exploring some of the analytic and theoretical issues which emerge when we attempt to identify instances of miscommunication and diagnose how they came about. The practical implications for workplaces are also discussed.

After evaluating previous approaches, the author proposes a comprehensive working model for analysing miscommunication or problematic discourse in workplace interaction which is based on a flexible multi-layered theoretical and methodological framework. The analytic approach taken is to apply the tools of sociolinguistic discourse analysis to data from actual interactions along with associated ethnographic information, in conjunction with a critical analysis of organisational communication practices and processes as seen from a community of practice perspective. A multi-dimensional intertextual approach such as this allows analysis of miscommunication and problematic talk at a number of different levels in order to relate what is happening sequentially and 'on-line' during particular interactions or sequences of interaction to factors such as social identity, group membership, team culture and other aspects of the wider communicative and socio-cultural context. 



\title{
1 Introduction The problem with workplace communication
}

\author{
Resolving a possible misunderstanding: \\ Jan: $\quad$ what- what happened to Marama? + was- \\ I presumed that Marama was going \\ was that MY misunderstanding? + \\ Heke: [drawls] $\mathrm{OH}$ \\ Jan: $\quad$ to the $+/ /$ ministry| \\ Heke: $\quad$ Iministry $\quad$ I I presumed she was going as well
}

\subsection{Rationale for the study}

Miscommunication is generally regarded as an 'occupational hazard' by people working in organisations. From a sociolinguistic perspective this is hardly surprising given the complexity of language and interpersonal communication in any social context. Moreover, in workplace settings, there is often a greater risk than elsewhere that ineffective or problematic communication will have visible and/or costly negative outcomes, for the individuals concerned or for the organisation; even in instances where communicative trouble or mishaps pass unnoticed, they may still resurface to create problems later. The potential consequences range from relatively minor and easily repairable interruptions to the smooth flow of work or communication between colleagues, as in the opening example above, through to more serious disruptions of productivity or workplace relations such as a case described in chapter 6 where a simple failure to clarify a verbal message led to a major production line outage in a factory. Even in cases where problems are avoided, or where a discussion results in constructive resolution of a tricky issue, misunderstandings and differences of opinion will inevitably take time, energy and relational skill to work through.

From an organisational perspective, a high level of individual competence in communication is a routine requirement nowadays for most jobs, and effective communication is identified as a critical factor in the efficient running of 
organisations in much contemporary management writing. At the same time, there is a widespread perception that communication problems and conflicts are endemic within organisations, and that communication practices need to be improved if workplaces are to function smoothly, safely and effectively. Management and legal case studies published in New Zealand and internationally, for instance, frequently refer to problematic dealings with so-called 'difficult' colleagues or clients, and to communication issues arising from linguistic or cultural diversity, from increased information and systems complexity, and from organisational change. Reported areas of concern are wide-ranging and can include aspects of spoken, written and electronic communication (both formal and informal), interpersonal and intergroup relations and conflict, as well as higher-level organisational communication processes and structures.

Precisely what is meant by the terms 'effective communication' or 'miscommunication' is, however, seldom clearly defined or articulated in such contexts. These terms tend to be used somewhat loosely by laypeople and workplace practitioners to gloss a range of issues which often go well beyond the usual scope of purely linguistic or discursive inquiry, even though communication may well be implicated as one factor. Training in various kinds of communication skills, or reviews and audits of communication processes and systems, are common responses to these issues in workplaces. Even where such interventions are appropriately targeted, they are often based on over-simplified assumptions about language and communication and how these operate in a socio-cultural context, rather than being grounded in linguistic, pragmatic or interactional analysis of how people actually communicate in work settings.

Academic perspectives on language and discourse therefore potentially have a great deal to offer anyone with a practical interest in improving the effectiveness of workplace communication. There is a very large body of research literature in this area, reflecting the amount of intellectual effort invested over many years in attempts to describe, theorise and deconstruct concepts relating to miscommunication and other aspects of problematic interaction. However, as detailed in later chapters, miscommunication and problematic discourse are so complex and many-layered that they continue to resist straightforward classification or definition, and certainly there is no unitary approach to studying these phenomena from within the academy. This 
diversity of perspectives and lack of coherence in the existing research base leaves considerable scope for further systematic description and theorisation of problematic communication in workplace settings, as well as raising questions about the "practical relevance" (Roberts 2001) of much of this research for workplace practitioners.

It is my proposition that no single theorisation or methodology can be expected to comprehensively address real world cases of workplace miscommunication and problematic talk in all their complexity, and that it is therefore necessary to apply a multi-layered intertextual approach to the study of these phenomena which incorporates multiple viewpoints, analytic methods and data sources. This thesis will explore the practicality and utility of applying such an approach by undertaking in depth analysis of interactional case studies from New Zealand workplaces in an attempt to identify more precisely where and how problematic communication develops and plays out, and to describe the range of discourse strategies used by participants to manage this 'occupational hazard'.

The next section of this chapter provides background information relevant to the research reported in this thesis, including a brief overview of the wider project which generated the observations that first triggered the particular investigation reported here, and within which it is embedded. The final section sets out the objectives and scope of this research, and outlines the remainder of the thesis.

\subsection{Background}

\section{Language in the Workplace Project}

The investigation reported in this thesis grew out of the Language in the Workplace (LWP) project, a wide-ranging and still ongoing programme of sociolinguistic research into interpersonal communication in New Zealand organisations based at Victoria University of Wellington. ${ }^{1}$ The LWP project first began in 1996 with two very broad goals: (1) to analyse the features of effective interpersonal communication in a variety of New Zealand workplaces from a sociolinguistic

\footnotetext{
${ }^{1}$ See Stubbe (1998a, 2001), Holmes and Stubbe (2003a) and the project website (LWP 2003) for further details about this project. The information about the LWP research provided in section 1.2 of this chapter and in chapter 4 is a summarised version of material from these sources.
} 
perspective; and (2) to explore the practical implications of these findings for New Zealand organisations. I was closely involved with the LWP project from its outset in my roles as Research Fellow and project manager from 1996 to 2003, sharing responsibility for initiating and planning the project and for leading the design and implementation of the data collection methodology, as well as collaborating on subsequent data analyses and maintaining an ongoing dialogue with research participants and end-users. $^{2}$

One of the motivations for initiating the LWP project in 1996 was the observation by the sociolinguists involved that there was a relative dearth of research based on data drawn from real life interactions in workplace contexts, which is after all where people conduct the day-to-day business of organisations. This gap was evident in both the organisational communication and sociolinguistic/discourse analysis literatures internationally, and in New Zealand settings. In the management field at that time, most reported studies, textbooks and training materials on organisational communication, almost without exception, used material derived from indirect sources such as self-report data, interviews with significant personnel, and anecdotal observations. Although there has been some degree of change in the decade since, most notably in some academic research within the field of organisational studies (see chapter 2), this observation still holds true for the majority of commonly used textbooks and training materials dealing with organisational and interpersonal communication (see chapter 2.3.6).

Even in applied linguistics, discourse analysis and related fields, there was little extant research in the mid to late 1990s which focused on how people actually communicate verbally with work colleagues on a daily basis, and how they use language to manage the inevitable tensions between their various professional and social roles. Despite a growing interest in researching workplace interaction at the time the LWP project was first set up (see for example Drew and Heritage 1992a; Roberts, Davies and Jupp 1992; Boden 1994; Tannen 1994, 1999; Sarangi and Slembrouck 1996; Bargiela-Chiappini and Harris 1997a, 1998; Hunston 1998; Sarangi and Roberts 1999b), much of the available linguistically-oriented research on institutional or organisational discourse at that time tended to concentrate on rather specialised 'frontstage' contexts such as classrooms, courtrooms, and doctor-patient

\footnotetext{
${ }^{2}$ Since 2003 I have retained links to the LWP in my capacity as a Research Associate.
} 
interactions (Sarangi and Roberts 1999a), and once again there was virtually nothing from New Zealand settings (see also chapter 3). In addition, the workplace data in most studies typically comprised more formal interactions in relatively static settings such as meetings or interviews. Research drawing on data from offices, where people move around to talk to different colleagues, or from factories, where many jobs entail continuous movement and considerable machinery noise, was (and remains) relatively rare. $^{3}$

The research data analysed and presented as part of the present study were collected and archived between 1996 and 2003 as part of the LWP corpus of workplace interactions. By 2003, this corpus comprised approximately 1500 interactions recorded in a wide range of New Zealand workplaces, including government departments, factories, small businesses, semi-public or non-government organisations (NGOs), and private commercial organisations. The interactions recorded include business talk and social talk, informal talk and meetings of many different sizes and kinds, with participants from a wide range of different levels in the workplace hierarchy. For all workplaces, varying types and amounts of ethnographic information were also gathered to assist with interpreting the interactional data. In some cases, more systematic structured observations of work patterns were undertaken before recording commenced. Wherever relevant, written documentation was also collected to provide background for the spoken communication, including agendas and minutes of meetings, reports, notices, manuals, production documents, and so on.

As well as providing a unique collection of naturalistic data, the methodology used to collect the LWP data corpus was also ground-breaking and innovative in a number of other respects. Of particular relevance here, the participatory methodology was designed to give informants the maximum possible control over data collection, and regular opportunities for dialogue between researchers and workplace participants were also built in to the research process, sometimes as an ethnographic component of the fieldwork, sometimes framed explicitly as action research. The interactional

\footnotetext{
${ }^{3}$ Two important exceptions to this general observation include a large study of spoken discourse in multicultural workplaces in Melbourne, Australia (Clyne 1994; Neill 1996), and an anthropological linguistic study of interactions between US and Japanese workers in a Texan car factory (Sunaoshi 1999, 2005). The existing literature is reviewed more fully in chapter 3 .
} 
and ethnographic data in this corpus thus provide a very rich and appropriate baseline resource for investigating miscommunication and problematic talk in New Zealand workplace settings. Chapter 4 will provide a more detailed description of the data sets from which the case studies in this thesis are drawn and the field methodologies employed in their collection.

Feedback obtained from organisations recruited as research partners in the first year of the project led to the articulation of two further, more specific and applied objectives for the LWP project. These became an ongoing focus of the wider research programme, and are also closely linked to the research reported in this thesis. The first of these more specific objectives was to identify and diagnose possible causes of miscommunication in verbal interaction in New Zealand workplaces, particularly in relation to gender and cultural issues (e.g. Stubbe 1997a, 1998b; Holmes, Stubbe and Vine 1999; Stubbe 2000a; Holmes and Stubbe 2003b 2003c; Holmes and Marra 2004). The second objective was to develop a set of communication evaluation and development resources based on the findings of the LWP research to help individuals and teams in workplaces critically reflect on their own practices within an action learning framework in order to improve the effectiveness of their communication (e.g. Stubbe and Marra 1999; Holmes et al 2000; Stubbe 2000b, 2002; Stubbe and Brown 2002; Jones and Stubbe 2004; de Bres 2009).

These analytic themes also represent two major strands of my personal research platform in the fields of applied sociolinguistics and interaction studies since 1996. In addition to the references already cited, this work has included a number of studies focussing on aspects of problematic talk in organisational settings undertaken as part of the LWP programme (e.g. Stubbe 1998c, 1998d, 1999a, 2000c; Stubbe and Holmes 2000; Stubbe, Holmes et al 2001; Daly et al 2003; Stubbe, Lane et al 2003), as well as in other institutional contexts including healthcare and call centre interactions (e.g. Stubbe 1997b; Stubbe, Dew et al 2006; Dew, Stubbe et al 2007; Dowell, Macdonald et al 2007; Dew, Dowell et al 2008; Dew, Plumridge et al 2008; Stubbe, Dowell et al 2008; Stubbe and Dew 2009; Stubbe, Dew et al 2009; Weatherall and Stubbe 2009; Dew, Stubbe et al 2010, Stubbe, Dew et al fc). This previous work has been drawn on and referenced as applicable in this thesis. 


\section{Motivation for the present research}

In the early stages of the research partnership between participants and LWP analysts, an intriguing mismatch became apparent between the common sense lay perceptions and experiences reported by the workplace participants and what the researchers thought they were seeing (or not) in the interactional data. This was the initial puzzle that triggered the particular line of inquiry reported in this thesis

During the initial briefings, follow-up interviews and workshops which were conducted as part of the first phase of the LWP Project (see chapter 4), participants regularly reported that communication problems of various kinds were widespread and of ongoing concern, affecting relationships between colleagues in the same teams or in different sections of an organisation, between management and staff, between staff and external contacts, or between different gender and ethnic groups. They generally attributed these to instances of miscommunication or to differences in communication style, and thus sought information and advice from the research team about ways they might identify and address these perceived problems as a means of improving productivity and workplace relations.

However, obvious or at least readily identifiable instances of miscommunication appeared to be far less common in the actual interactional data than these informant reports led the researchers to expect. In fact, the initial analyses conducted by the project team, supported by participants' self-assessments of the same interactions, indicated that on the whole people appeared to do a remarkably good job of communicating effectively with their colleagues on a day-to day basis, as measured by the achievement of their stated and implicit transactional goals, and by the maintenance of smooth working relationships during particular interactions or sets of interactions. What a close analysis of the discourse did reveal, however, was the skilful use of a variety of discourse strategies which functioned to avoid, minimise or repair potential miscommunication or other tricky interactional issues in everyday workplace interactions. It was clear from the ethnographic evidence and feedback sessions in workplaces that for most people this occurred largely below the level of consciousness. Nevertheless, the interactional evidence showed clearly that people routinely used a range of direct and indirect strategies, including humour, small talk and a range of politeness strategies, to balance their relational identities and instrumental goals in particular contexts such as meetings, working sessions, or 
trying to get others to do things. Interactants could thus be observed to engage in a constant and delicate discursive balancing act as they attempted to get tasks done while at the same time managing their relationships with their co-workers (Holmes and Stubbe 2003a). This interplay between the imperatives of sometimes conflicting transactional and relational goals was especially foregrounded in those cases of miscommunication and problematic talk that were clearly identifiable in the data, and it was these "interactional dilemmas" (cf. Gill and Maynard 2006; Dowell et al 2007:347) that often made such interactions problematic in the first place.

This apparent mismatch between the detailed micro-level analyses of individual interactions and the anecdotal evidence of widespread communication issues reported by participants themselves became the starting point for the trialling of a reflective action learning model for communication evaluation and development (CED) in the participating workplaces. This model is predicated on the assumption that developing an increased awareness of the underlying discourse processes and structures in their interactions and having access to a set of tools to hone their observational and reflective skills would assist people in workplaces to manage their day-to-day communication more effectively. (See Jones and Stubbe 2004 and chapter 5 for a fuller discussion of the CED model and how this collaborative process evolved).

However, as this iterative research process continued, it became increasingly apparent that the researchers' and workplace participants' differing perceptions regarding the prevalence, nature and provenance of miscommunication and problematic talk were symptomatic of a more fundamental issue than a simple and arguably predictable discrepancy between lay and 'expert' understandings of these phenomena. Instead, they appeared, at least in part, to be artefacts or blind spots of the data collection methods and analytic frameworks being used by the researchers. Despite the richness of the early LWP data set by comparison with other studies, the original research design thus had some important limitations which seemed to be preventing the researchers from gaining a full appreciation of the complexities involved in miscommunication and problematic talk. This point will be discussed more fully in chapter 4 .

Firstly, there was a capture problem in that the interaction data collected in a given site represented only a tiny proportion of the talk that actually occurred in a given 
workplace during the data collection period, and usually consisted of a collection of fairly randomly selected one-off recordings. These data snapshots were seldom linked by topic, and typically included a wide range of interactants, settings and activity types. Given the decontextualised, time limited and partial nature of each sample, in hindsight therefore, it is unsurprising that this initial phase of data collection did not capture as many overt or readily identifiable instances of miscommunication as might have been expected.

Secondly, as elaborated in chapters 3 and 5, there was also an identification problem arising from the sheer complexity of the phenomena in question, and the fact that the data actually collected often did not contain sufficient observable or identifiable traces to allow analysts to recognise an interaction as being in some way part of a problematic communication sequence. For instance, it is logically possible to identify potential problems at a number of distinct but interrelated levels. From the analysts' perspective, in some cases it was quite clear from the discourse context that a misunderstanding of some sort had occurred. However, we could just as easily fail to recognise more subtle instances of (potential) miscommunication unless these were pointed out by our informants. As organisational outsiders, the analysts clearly could not expect to have access to a great deal of contextual and historical information available to participants from their situated experience of a complex fabric of related interactions, documents and other types of shared institutional knowledge.

\subsection{Objectives and scope}

The observations above led directly to the proposition to be explored in this thesis, namely, that to comprehensively address real world cases of workplace miscommunication and problematic talk in all their complexity requires a pluralistic multi-layered intertextual approach which incorporates multiple viewpoints, analytic methods and data sources. This study will explore this proposition by means of an in depth analysis of interactional case studies from New Zealand workplaces. This analysis has three key objectives as summarised in Table 1 below, with primarily theoretical, methodological and practical (applied) relevance respectively. 


\section{Objective 1 (Theoretical):}

To systematically explore and problematise a set of relevant theoretical approaches to the concepts of miscommunication and problematic talk via empirical investigation of an existing corpus of naturalistic workplace data.

\section{Objective 2 (Methodological):}

To build the specifications for a methodological and analytic framework which is sufficiently spacious and robust to account for the intertextual and situated nature of miscommunication and problematic talk observed in this data set.

\section{Objective 3 (Practical/Applied):}

To identify and describe the range of communication problems that typically arise in these New Zealand workplace contexts, and the strategies used by the people in these workplaces to maximise the effectiveness of their communication and to prevent or repair miscommunication and problematic talk.

This thesis will consider the ways in which instances of miscommunication and problematic talk in New Zealand workplaces are instantiated in selected cases drawn from the Language in the Workplace corpus collected between 1996 and 2003. The study is positioned within a multidisciplinary space; as such it is based broadly within the disciplinary framework of interactional sociolinguistics, but also draws on concepts and analytic tools from a range of related styles of discourse and interaction analysis, with additional links to applied linguistics and organisational communication.

The analytic focus is on the problematic aspects of everyday or 'backstage' spoken discourse between co-workers in New Zealand workplaces, as opposed to public or 'frontstage' institutional or organisational settings such as professional-client interactions. The primary data examined therefore comprises naturally occurring talk-in-interaction collected as part of routine workplace activities, supplemented by a variety of ethnographic data, including information based on observations, interviews, workshops and other forms of dialogue with participants, and written or electronic texts related to the interactions analysed. 
The remainder of the thesis is structured as follows:

The next two chapters review the relevant literature pertaining to miscommunication and problematic talk in the workplace.

Chapter 2: Literature review (a). Theoretical perspectives on workplace discourse summarises the main theoretical and methodological frameworks which have been applied to the study of spoken discourse more generally in institutional and organisational settings.

Chapter 3: Literature review (b). Miscommunication and problematic talk provides an overview of how the concepts of miscommunication and problematic talk are defined and understood in the existing literature, and a selective summary of previous research into miscommunication and problematic talk relating specifically to workplace settings. This is followed by a discussion of the gaps and opportunities identified in the existing research which led to the formulation of the specific research questions set out at the end of chapter 3.

Chapter 4: Methodology. Analytic approach and research design describes, explains and justifies the methodological approach taken in this study, and provides details of the research design, including a description of the data set and the data collection and analytic methods used.

Chapter 5: Developing a working model. What exactly is miscommunication anyway? sets out the theoretical and practical principles underpinning the development of a working model of miscommunication and problematic talk, and outlines a proposed integrated analytic framework. Analyses of three exemplar cases from the baseline data set serve to illustrate and evaluate the utility of this heuristic framework in relation to the description of a range of different types of miscommunication and problematic talk identified in the original LWP project corpus, and the strategies used by workplace participants for managing these.

Chapters 6 and 7 present an in depth longitudinal case study of communication and miscommunication in a factory team, using data collected specifically for this purpose in a later development of the LWP methodology. These chapters further tease out the theoretical and methodological issues canvassed in earlier chapters, but also address more substantively the third objective of the research, by presenting a “thick description" (Sarangi and Roberts 1999a) of this particular team's 
communication patterns within a community of practice framework, thus extending the analytic framework presented in chapter 5. This case study is divided into two parts.

Chapter 6: (Not) 'getting the message across'? Problematic talk on the factory floor provides an illustrative analysis of the team's communication practices and the issues that arise in the course of a typical shift, as well as examining the discursive strategies used by team members to optimise the effectiveness of their communication.

Chapter 7: Managing the contradictions. The discursive strategies of the team leader focuses on the intertextual and interdiscursive strategies used by the team coordinator for managing miscommunication and problematic talk in this setting and for optimising the team's communicative effectiveness, and adds a further critical dimension to the analysis.

Chapter 8: Problem or paradox? The elephant in the room, as the concluding chapter draws together the threads of the arguments and evidence put forward in the earlier chapters, evaluates the theoretical, methodological and practical implications of the findings, and outlines possible directions for future research. 


\title{
2 Literature review (a) Theoretical perspectives on workplace discourse
}

\begin{abstract}
A group of blind men heard that a strange animal, called an elephant, had been brought to the town, but none of them were aware of its shape and form. Out of curiosity, they said: "We must inspect and know it by touch, of which we are capable". So, they sought it out, and when they found it they groped about it. ... (T) he first person, whose hand landed on the trunk, said, "This being is like a drain pipe". For another one whose hand reached its ear, it seemed like a kind of fan. ... (A)nother person, whose hand was upon its leg, said, "I perceive the shape of the elephant to be like a pillar". And ... the one who placed his hand upon its back said, "Indeed, this elephant is like a throne". Now, each of these presented a true aspect when he related what he had gained from experiencing the elephant. None of them had strayed from (its) true description ... yet they fell short of fathoming (its) true appearance ...
\end{abstract}

Jainist Parable of the Blind Men and the Elephant (Hughes, 2005: 590-1)

\subsection{Introduction}

The wider language, communication and social sciences literature includes a large volume of published research relating in some way to workplace discourse and/or to miscommunication and problematic talk, which is potentially relevant in some way to the research topic being addressed here. However, this body of work encompasses such a diversity of perspectives, research domains and settings that, taken as a whole, it can only be characterised as being a rather fragmented and non-cohesive field of inquiry. As the literature review in this and the following chapter will demonstrate, the range of theoretical frameworks and methodological approaches available has made it possible for researchers to 'thin slice' and fruitfully investigate many specific aspects of communication and miscommunication through different lenses and to do so from a number of angles, both in the workplace and in a variety of other settings. Nevertheless, if the objective is, as here, to explore situated examples of this complex phenomenon in a holistic way, we are left with a problem somewhat akin to the blind men's exploration of the elephant in the Jainist parable quoted above. Namely, no single body of work or theorisation can, by itself, provide a sufficiently comprehensive account of miscommunication and problematic talk at work to 
satisfactorily address the multi-faceted real-world and theoretical problems set out in the previous chapter, yet there are important and valuable insights to be gained by considering a multiplicity of viewpoints.

A comprehensive review of all the potentially relevant literature here is obviously beyond the scope of this thesis, and given the size and diversity of the field, it would in fact be impossible to provide this in any reasonably sized work. However, although it is inevitably highly selective, a fairly wide-ranging overview is provided here and in chapter 3 to lay out the theoretical and empirical foundations for the present study. This chapter will background the theoretical perspectives and methodologies most commonly used by previous researchers to frame and analyse spoken discourse in workplace settings. Chapter 3 will focus more specifically on miscommunication and problematic talk: how these have been defined and conceptualised, and the kinds of findings generated by these approaches in workplace settings.

\subsection{Theoretical frameworks}

Workplace communication has been researched within various theoretical paradigms, drawing on different disciplinary frameworks and epistemological stances, and including both theoretical and applied perspectives. Relevant bodies of work are located in the humanities and social sciences (including various branches of linguistics, pragmatics, discourse analysis, communication studies, sociology, anthropology and social psychology), in organisational studies (including management, organisational communication, organisational psychology, genre and activity theory, systems safety and quality assurance), in the fields of vocational and professional education, as well as in various interdisciplinary spaces. The methodological approaches within this field of inquiry are equally varied, ranging widely from 'micro' perspectives such as the fine-grained analysis of natural interaction, through various observational or ethnographic methodologies, to more 'macro' or 'big-picture' explorations of communicative systems and meta-discourses within the realms of social and organisational theory. This chapter provides an overview of the main epistemological and theoretical perspectives within which 
research on workplace communication has typically been framed and the key methodological approaches derived from these.

\subsubsection{Epistemological positions}

Most language and communication research is grounded in one of two broad epistemologies or philosophical 'ways of knowing'. The objectivist/realist position assumes the existence of an objective and universal reality or truth which is knowable and able to be directly experienced. The constructionist/intersubjectivist stance on the other hand holds to the notion that ideas and concepts are not universal, and that 'truth' or 'reality' reside in multiple perspectives constructed from people's mutual experience or interactions. These epistemologies in turn align broadly with three main sets of theories or models of communication: (i) structural, functional and cognitive/behavioural theories (ii) interactionist and intersubjectivist theories, and (iii) critical theories which combine elements of both perspectives (Littlejohn 2001).

Structural, functional and cognitive/behavioural theories tend to be associated with positivist and essentialist perspectives based on the objectivist notion that "meaningful reality exists as such apart from the operation of any consciousness" (Crotty 1998:8); communication and human meaning are thus seen as largely determined by existing social structures and/or individual cognitive structures. Approaches fitting broadly within these frameworks include variational sociolinguistics, the ethnography of communication, intergroup or cultural difference theories, skills-based communicative competence models, speech act theory, Gricean pragmatics and politeness theory. Traditionally these approaches have also typically been associated with linear transmission ('sender-receiver') or psychological ('encoding-decoding') models of communication. Such models assume that words or other signs transparently and unproblematically represent the concepts, feelings or intentions referred to by a speaker according to a pre-determined set of rules or implicatures (Trenholm 1999, 2010), and tend to focus on hearer-based interpretation and speaker-based (re)presentation of meanings. ${ }^{1}$ As Littlejohn (2001) points out, a

\footnotetext{
${ }^{1}$ It should be noted, however, that over the past decade in particular there has increasingly been a 'discursive turn' within some of these theoretical domains, namely, a movement towards postmodernist and interactionist perspectives and away from positivist epistemologies.
} 
fundamental point of difference for this set of theories is that thinking is seen to precede its articulation in language, rather than taking place in or through language.

Post-modernist intersubjectivist and interactional theories question the idea of social or linguistic categories as given, and instead take a more relativist, social constructionist view of reality as "determined not by empirical observation but by the categories (linguistic and conceptual) we possess to define it" (Unger 1989:2). There is an emphasis on the dynamic aspects of interaction, and the constantly changing and developing nature of social identities, social categories and group boundaries (Weedon 1987; Butler 1990). Examples of these approaches include conversation analysis, interactional sociolinguistics, communication accommodation theory, and post-structural social identity models. Social constructionist approaches to interpersonal communication assume that the unfolding sequential structures in an interaction and participants' shared knowledge provide the means by which participants jointly and dynamically construct a particular social order and come to a mutual interpretation of what is going on. Hence in these theories, social structures and meanings are situated in, built, reproduced, and transformed via social interaction and communication, which are framed as being instrumental in the creation of our social worlds.

Critical theories, such as the various brands of critical discourse analysis, discursive psychology and feminist post-structural discourse analysis, can be seen to draw on both objectivist and constructivist perspectives, but all are characterised by their meta-analytic interest in agency and power relations in a given communicative context. Critical theorists are usually sceptical of claims or appearances of equality and consensuality in interactions, and these theories tend to have an ideological focus on deconstructing the ways in which hegemonic power is achieved and/or resisted via dominant and subversive discourses. Within this paradigm, discourse is described and explained as "an instrument of control as well as communication" (Kress and Hodge 1979); as such, discourses are never neutral, and social structure is seen as existing, not outside discourse, but within it as "shared knowledge" (Berger and Luckman 1966, cited in Candlin 1987). 


\subsubsection{Definitions of discourse}

In its broadest sense, discourse can be defined as any aspect of language in use, and may include both spoken and written language. Perhaps unsurprisingly therefore, there is a breathtakingly wide array of analytic approaches to the study of discourse based in many different disciplines (Stubbe et al 2003:351). These include the various models referred to above, in addition to others such as social semiotics and systemic-functional linguistics, proxemics, multimodal discourse analysis, and various flavours of rhetorical, stylistic, semantic and narrative analysis. Exactly what is meant by the term 'discourse' in the literature therefore clearly depends on the writer's disciplinary and theoretical orientation and their focus of inquiry.

As used by linguistic discourse analysts and pragmaticists, interactional sociolinguists, ethnographers, and ethnomethodologists (i.e. researchers who engage in micro-analysis of various kinds), the term 'discourse' usually refers to a single spoken interaction or written text in a specific social context, or to a particular activity type, genre or unit of interaction such as conversations or meetings. Critical discourse analysts and others working within a postmodernist social theory framework (i.e. researchers who work at a macro-analytic level) apply a more complex definition; even where their main focus remains on the linguistic analysis of empirical texts in context, which is by no means always the case, they understand discourse(s) as a multi-layered form of action (van Dijk 1990:164). ${ }^{2}$ These approaches draw heavily on the more abstract Foucauldian view of discursive practice and orders of discourse, in which language and society partially constitute one another (Grimshaw 1981; Foucault 1982). In this framework the term 'discourse' refers to a subset of or the totality of interactions in a given domain such as medical, media or other institutional discourse. Such discourses are marked by intertextuality and interdiscursivity (Fairclough 1993), and are always related to other discourses, both historically and synchronically (Fairclough and Wodak 1996).

However, despite sometimes quite fundamental differences in perspective, all the broadly discursive approaches to workplace communication considered here share two basic assumptions: firstly, that discourse is in some way interdependent with

\footnotetext{
${ }^{2}$ These two broad 'levels' of discourse are sometimes characterised as discourse with a small ' $d$ ', and Discourse(s) with a large 'D', reflecting their concern with micro- and macro-level analysis respectively (see Weatherall et al in press; 2.4 below).
} 
social life, and that its analysis therefore of necessity intersects with meanings, activities and systems outside of itself; and secondly, that discourse should be viewed as a system in its own right, a socially and culturally organised way of speaking or writing, by means of which particular functions are realised or constructed (Schiffrin 1994:31).

There are also many areas of overlap and cross-fertilisation between different approaches, and in practice, the boundaries between them can be fuzzy as discourse analysts and communication scientists often take an eclectic approach, applying elements of one or more models or disciplinary frameworks, as relevant to their particular research objectives. As Schiffrin (1994:419) points out, researchers from one tradition often draw quite explicitly on the insights and methods of other traditions, resulting in rich interactions such as those between conversation analysis and the ethnography of communication (e.g. Moerman 1988; Gumperz 1982a, 1982b), and between pragmatics and conversation analysis (e.g. Levinson 1983, 2005). Interactional sociolinguistics (IS) and feminist post-structuralist discourse analysis (FPDA) are two cases where such a cross-fertilisation of approaches has become formalised into a theoretical and methodological approach in its own right (see Gumperz 1999; Stubbe et al 2003; Weatherall et al in press, for more detailed discussion of these points).

The remainder of this section summarises the key features of the main approaches to discourse and communication in workplace settings which have been considered and drawn on in the course of the present study, including broadly linguistic, social science, critical and organisational paradigms. Because my interest here is specifically on methodologies commonly used to research spoken interaction or communication in workplace contexts, this overview does not include detailed reference to many other frameworks which are beyond the scope of the present study, including those focusing exclusively on written or electronic and nonsynchronous communication, and models based in cognitive psychology, neuropsychological and psychoanalytic theories, social semiotics and rhetoric.

Each of the specific approaches selected for further explication offers a distinctive perspective on social interaction and a different analytical framework for examining patterns of discourse and communication in workplaces. At one level each approach can be distinguished by the way in which it relies on the epistemological stances and 
broad conceptualisations of communication outlined above. However, here the different approaches are grouped primarily on the basis of their analytic focus and the methodologies typically adopted in each case under six umbrella headings: conversation analysis, pragmatics, sociolinguistics, discursive social psychology, critical discourse theory and organisational/ communication studies. These groupings are presented in an order approximating their position along a continuum from micro-level to macro-level analysis of discourse and its context(s).

\subsection{Methodologies}

\subsubsection{Conversation Analysis}

Conversation analysis (CA) has its roots in sociology, specifically ethnomethodology, although it is increasingly regarded as a separate discipline in its own right with connections to linguistics, sociology, anthropology and psychology. Practitioners of CA see talk-in-interaction as an inherently social process which is "deeply involved in the production and maintenance of social institutions of all kinds" (Hutchby and Wooffitt 1998:37). CA is the epitome of a social constructionist model of communication as joint activity (Sacks 1984), and focuses on how a wide range of actions and activities, such as conversational openings and closings, (non)alignment, agreement and disagreement, medical diagnoses, narratives and so on, are interactionally achieved.

CA is "very circumspect about premature theorising and formalisation" (Stubbe et al 2003:356), and also "provides a strict empirical framework for analysing in detail the way participants jointly construct the interaction, and at the same time constitute the context, including participants' identities, utterance by utterance" (Stubbe et al 2003:376). Like pragmaticists (see 2.3.2 below), conversation analysts' primary interest is in the actions performed by talk rather than the linguistic forms or structures in their own right, but in CA, these actions are seen as co-constructed, with each utterance understood as a step in a joint activity. This is in contrast with the approach taken in pragmatics which typically analyses one utterance at a time, and attributes certain intentions to speakers on the basis of their use of a particular discourse strategy. 
The hallmark of CA is its methodological approach, which is grounded in extremely intensive and rigorous micro-analysis of naturalistic data obtained by recording and transcribing naturally-occurring interactions in very close detail (Ten Have 2007). Unlike sociolinguistic and ethnographic approaches, CA does not seek to take account of contextual information external to the interaction itself unless participants demonstrably orient to it in some way - essentially the interaction is the context. CA focuses on sequential structures in conversation, which are seen to provide the means by which participants co-construct a particular social order and come to a shared interpretation of what is going on in an interaction as it unfolds turn by turn.

Within conversation analysis, talk-in-interaction is seen to be highly structured, for instance in terms of rules for turn-taking, and preference organisation. or constraints on the ways sequences can unfold. However, these rules and preferences are seen to operate below the level of conscious awareness, are always locally occasioned, and “... are not invariant descriptive rules in the linguistic sense... but rather normative and interpretative" (Stubbe et al 2003:355); as such, they provide a reference point for participants to treat actions as unremarkable or accountably deviant and thus requiring interactional repair or justification.

The CA enterprise is essentially aimed at uncovering these rules and structures via empirical analysis, and since the first studies in this tradition were published in the late 1960s and early 1970s (e.g. Sacks 1967; Schegloff 1968, 1972; Sacks 1972a, 1972b; Sacks et al 1974), a large body of work comprising studies of many specific aspects of interaction has been built up into a well-integrated theoretical model of talk-in-interaction. From the late 1970s an applied version of CA (e.g. Drew and Heritage 1992a; Heritage and Maynard 2006) has developed in parallel with what Ten Have (2007) terms the more "pure" theoretically-oriented studies. These applied CA studies focus particularly on institutional interactions with the aim of demonstrating how institutions are "talked into being" (Heritage 1984:290). This strand of CA research has proved to be very productive indeed over recent years, as seen for example in the extensive literature on CA in clinical encounters (e.g. Heritage and Maynard 2006; Pilnick et al 2010). The concepts and techniques developed within CA have also influenced discourse analysts working within other paradigms such as pragmatics, interactional sociolinguistics, discursive psychology, feminist post-structural discourse analysis and even organisational communication; 
in these cases elements of the CA approach, in particular its analytic tools, are often used in conjunction with other forms of ethnographic and contextual analysis.

\subsubsection{Pragmatics and politeness theory}

Pragmatics, or "the science of the unsaid" (Levinson 2005:433), was originally based in the philosophy of language. It is a socially oriented form of discourse analysis, concerned at the broadest level with "meaning in use or meaning in context"; in other words, with the illocutionary force of utterances as opposed to their abstract or literal meaning (Thomas 1995:1-2). A number of different models exist within pragmatics, including speech act theory, functional linguistics, conversational implicature and various theories of politeness (see below). However, all share a basic linguistic orientation to discourse as a structured level of language which, like grammar, semantics and phonology, is assumed to be "based for the most part on quite regular and abstract principles" (Levinson 1983:53) which inform the context-dependent negotiation of meaning by speakers and hearers.

Pragmatics has traditionally fitted into the rubric of structural/functional/cognitivist theories of language and communication, in that it is assumed that the meaning of an utterance in a given context can be interpreted in the light of a set of general conversational maxims, or principles relating to assumptions of 'cooperativeness' and 'relevance' (e.g. Grice 1975, 1981; Leech 1983). These universal rules form the basis for very specific inferences by hearers about the intended meanings of utterances. Pragmatics as a field is currently less unitary in this regard than it once was (see also note 1 above and the discussion of recent trends in Politeness Theory below). Nonetheless, pragmaticists of various ilks still concern themselves primarily with the processes of self-presentation, interpretation and inference which people use strategically to maintain discourse and to make it understandable and relevant at a very localised level of interaction (Roberts et al 1992:72). It is assumed that there is no one-to-one correspondence between linguistic form and function, and communicators are seen to operate strategically, with utterances open to multiple interpretations depending upon the presuppositions and goals of the participants (see also 3.2.2).

Politeness Theory has been a major preoccupation of pragmatics for the last 25 years (Thomas 1995:149). Classic models of politeness link a focus on speaker/hearer 
intention and interpretation to a sociological and anthropological concern with how interaction relates to social structures and culturally relative norms; "strategic message construction" is thus seen as "the key locus between language and society" (Brown and Levinson 1978). There are a number of specific models within the politeness theory paradigm involving "face-management" (Brown and Levinson 1987), "conversational maxims" (Leech 1983), and a "conversational contract" approach (Fraser 1990). The study of conflict and argumentation (e.g. Grimshaw 1990; Stein 1997) and misunderstandings (e.g. Bazzanella 1999; Dascal 1999; Weigand 1999; House et al 2003) in conversational discourse is another important strand of pragmatics research.

Functionalist pragmatics and politeness theories have been especially influential within sociolinguistics, for instance in the study of gendered language use, and to an extent also in communication studies, and have been widely applied to practice in the areas of second language learning, intercultural communication and organisational communication. Intercultural pragmatics in particular has provided a productive framework for research into language teaching and communication in multicultural workplace and institutional contexts (e.g. Clyne 1994; Neil 1996; Willing 1997; Moss and Roberts 2005; see 2.3.3 below), while politeness theory has been widely applied to studies of status and power relations in organisations (e.g. Morand 1996a, 1996b; Holmes and Stubbe 2003a; Mullany 2006; Schnurr et al 2007).

However, the utterance level focus of traditional pragmatics models, their reliance on the analysis of idealised and elicited examples, and their emphasis on building predictive descriptive models have increasingly been challenged as more language and communication researchers turn to post-structuralist paradigms. There is a trend currently well underway for many pragmatics researchers to adopt a social constructionist epistemology based on the analysis of naturally-occurring talk-ininteraction, thus acknowledging the situated co-constructed nature of utterances within a discursive sequence (Stubbe 2009). Developments in politeness theory (e.g. Eelen 2001; Mills, 2002; Locher and Watts, 2005; Haugh, 2007;), and language and gender research (e.g. Bergvall et al 1996; Holmes and Meyerhoff, 2003; Coates 2004; Weatherall et al in press) over the past 15 years provide two particular instances of this substantive epistemological shift. 


\subsubsection{Sociolinguistics}

Within sociolinguistics, the two approaches most commonly applied to the study of workplace discourse are the ethnography of communication and interactional sociolinguistics. A third approach which has also gained influence as sociolinguistics has moved away from its positivist roots and towards more situated interactionist research models is the community of practice model.

\section{Ethnography of communication}

The ethnography of communication is a functional approach to discourse first developed by sociolinguist Dell Hymes (1974). It is based in anthropology and linguistics, and seeks to analyse localised patterns of communication as part of cultural knowledge and behaviour, placing particular emphasis on how and for what purposes language is used in particular contexts. This approach recognises both the diversity and cultural relativity of communicative practices which exist in human societies, and the fact that these are an integral part of what members of a particular (sub)-culture know and do. A central construct is the notion of communicative competence which comprises the knowledge speakers need to communicate appropriately within a particular speech community, and the skills they need to make use of it (Saville-Troike 2003:2). Since the 1980s an extensive literature on workplace communication has emerged from this tradition with its roots in the study of socially and culturally bounded groups (Sarangi and Roberts 1999a:26), and this approach remains influential within anthropological linguistics, sociolinguistics and communication studies more generally. Observational and other ethnographic techniques are traditionally the main tools used, rather than direct analysis of spoken discourse.

\section{Interactional sociolinguistics}

Linguistic anthropologist John Gumperz is the most influential proponent of interactional sociolinguistics, which has similar origins in anthropology, sociology and sociolinguistics to the ethnography of communication, and shares its concern with the interaction of culture, society and language (Gumperz and Hymes 1972). The notion of cultural relativity is also of central importance in this approach, which has been further enriched by the concept of framing first developed by sociologist Erving Goffman (1974). However, interactional sociolinguistics focuses much more explicitly on the social and linguistic meanings created during actual interaction, and 
in this way also draws on some of the principles and analytic tools of conversation analysis and pragmatics. Participants are observed to make inferences about one another's communicative intentions and goals based on a wide array of verbal and non-verbal contextualisation cues that form part of cultural repertoires for signalling meaning, and which can be discovered only through the analysis of actual utterances (Schiffrin 1994:12).

In this way interactional sociolinguists attempt to "bridge the gap" between top-down theoretical approaches which privilege "macro-societal conditions" in accounting for communicative practices (see below), and those such as conversation analysis which provide a "bottom-up" social constructivist account by focussing on spoken interaction as "the real world site where societal and interactive forces merge" (Gumperz 1999:453-4). Analysts working within this paradigm have in the past been interested mainly in the critical analysis of (problematic) discourse processes in "strategic" research sites characterised by status and power inequalities between the participants (Roberts et al 1992). Archetypal examples include studies of intercultural interaction (e.g. Gumperz 1982a, 1982b) male-female communication (e.g. Kendall and Tannen 1997; Tannen 1999), (inter-ethnic) meetings and interviews in organisational contexts (e.g. Drew and Heritage 1992a; Roberts and Sayers 1987; Mumby and Clair 1997), as well as research in other domains such as medical interactions (e.g. Moss and Roberts 2005; Hamilton and Britten 2006; Dew, Dowell et al 2008) and general workplace interaction (e.g. Hunston 1998; Holmes and Stubbe 2003a).

\section{Communities of practice}

The discourse-oriented communities of practice model which has developed within sociolinguistics fits broadly within the paradigm of discursive social identity theory (e.g. Weedon 1987; Butler 1990; see 2.3 .5 below), and has been adapted from organizational theories of "situated learning" (Lave and Wenger 1991; Wenger 1998, 2000; see also 2.3.6 below), in the first instance as a way of accommodating a social constructionist turn in work on language and gender previously based in variational sociolinguistics, socio-cultural linguistics and pragmatics (e.g. Eckert and McConnell-Ginet 1992, 2003; Holmes and Meyerhoff 1999). Within this context, a community of practice is characterised as "an aggregate of people who come together around mutual engagement in an endeavor ... defined simultaneously by its 
membership and by the practice in which that membership engages" (Eckert and McConnell-Ginet 1992:464). These practices might be "(w)ays of doing things, ways of talking, beliefs, values, power relations ... (that) emerge in the course of this mutual endeavor" (ibid:464). The community of practice model offers a sophisticated conceptual framework which, when combined with ethnographic and interactional methodologies, accommodates a situated focus on naturally occurring social groups and their discourse. As such, it has the potential to provide important insights into the way people interact within organisations. Further explication of this model is provided as part of the case study analysis in chapter 6 below.

\subsubsection{Social psychology}

Communication Accommodation Theory and Discursive Psychology are two approaches from the domain of social psychology which have been especially influential in the study of communication in workplace settings, sometimes in the case of the latter, in conjunction with other analytic frameworks such as conversation analysis, narrative analysis or critical discourse analysis.

\section{Communication Accommodation Theory}

Communication Accommodation Theory (CAT) has grown out of the work of social psychologist Howard Giles and his associates on language and intergroup behaviour (e.g. Giles and Coupland 1991; Gallois and Giles 1998; Williams 1999). This approach to discourse analysis is primarily concerned with establishing the critical role of language in maintaining and developing group identity, and in the social evaluation of speakers. CAT is a socio-psychological model, which encourages the analyst to take account of the broader societal context within which an institution or organisation is operating, as well as the more detailed social context of a particular speech event. It thus provides a means of exploring the influence of the type of organisation on the verbal interaction patterns of particular groups, as well as a framework for analysing in detail the effects of the particular contributions made by individuals in a specific interaction. As a social constructionist approach, CAT explores the ways in which participants construct and maintain their own and others' professional identity within an organisational context, for example, through congruent verbal behaviour, as well as the ways in which they sometimes challenge and undermine those constructions (e.g. Watson and Gallois 1999; Gardner and Jones 1999; Ladegaard 2009). 


\section{Discursive Psychology}

Discursive Psychology is also situated within social psychology, but, unlike CAT, this approach directs an explicitly critical and post-structural analytic lens onto the practices and resources used to justify and rationalise social behaviour. Within this framework, spoken and written texts are used to investigate psychological phenomena such as identity, attitudes and prejudice (e.g. Wetherell and Potter 1992; Edwards 2004; Edwards and Potter 2005), in particular the ways in which discourses, interpretative repertoires and practical ideologies maintain and reproduce social inequalities (Stubbe et al 2003). Discursive psychologists draw to varying extents on aspects of pragmatics, ethnomethodology and conversation analysis and postfeminist critical theory (e.g. Potter and Hepburn 2003; Wilkinson and Kitzinger 2006; Weatherall et al in press), but issues like the degree to which context can defensibly be included in analysis of interaction (Schegloff 1997; Wetherell 1998) and the role of cognitive processes (Potter 2005:743), remain as areas of debate and differentiation.

Within discursive psychology, language and communication are seen as "the site of the social" rather than as the target of analysis in their own right (Stubbe et al 2003:379), and the main emphasis is therefore on discovering patterns of language use in aspects of discourse such as recurring themes and argument structures in order to deconstruct stances, biases and dilemmas of stake. The focus is often, though not always, on texts at a more macro-level, and much use is made of elicited interview data, as opposed to the focus on naturalistic data in the other approaches discussed thus far. As noted by Hepburn and Wiggins (2005a), discursive psychology is increasingly being applied in institutional settings (e.g. Wetherell et al 1987; Riley 2002; Potter and Hepburn 2003; Kitzinger 2005a; Auburn 2005; Hepburn and Wiggins 2005b). This latter work builds on conversation analytic studies of institutional interactions (see also 2.3.1 above), but with a focus on some quite specialised settings and highlighting the "psychological business" that is characteristic of institutions such as therapy, education, focus groups and court cases (Potter 2005:745).

\subsubsection{Critical discourse theory}

The asymmetrical power and intergroup relations which are an unavoidable aspect of workplace interaction provide a fruitful territory for critical discourse approaches. 
Two of these, critical discourse analysis and social identity theory which have been especially influential, are briefly considered here.

\section{Critical discourse analysis}

The European tradition of critical discourse analysis (CDA) is based on the work of Continental theorists such as Habermas and Foucault. It focuses explicitly on exploring how power and ideology are manifested in discourse, and on the linguistic aspects of social and cultural processes and structures (e.g. Fairclough 1992a, 1992b, 1995; Wodak 1996; Weiss and Wodak 2003). As in other forms of critical discourse theory, discourse is seen as a form of social practice through which hegemonic power and dominance structures are (re)produced, and its practitioners often have an “overtly political agenda” (Kress 1990:85) with roots in Marxist philosophy.

Power is always a relevant dimension in any CDA work: it is assumed that "people habitually enact, reproduce and sometimes resist institutional power relationships in the ways they talk and write" (Holmes and Stubbe 2003a:100). Power in organisations manifests itself in interpersonal interactions, as well as in hierarchies and in access to specific information and discourses (Wodak 1996:12), and can take a number of different forms. For instance, Dwyer (1990) identifies the following four types of power which all allow someone to influence or control the behaviour of others within an organisation: a) "authoritative power", based on a "person's position or role within an organisation" (374); b) "expertise power", based on an individual's particular skills and strengths; c) "reward power", when a person or group has power over others because they have control over resources that others want; and d) "coercive power", when person uses "force, emotional or physical ... to keep others in line" (375). More powerful individuals may adopt "oppressive" (direct or overt) and/or "repressive" (indirect or covert) strategies to gain compliance or cooperation from others (Pateman 1980). All these types of power involve "coercive power" (Fairclough $(1989,1995)$ in situations where one individual or group has "power over" others (Ng and Bradac 1993; Yeatman 1994). By contrast, "consent power" (Fairclough 1989), or "power to" (Ng and Bradac 1993; Yeatman 1994), is nonhierarchical in nature and includes sub-categories such as "consultative power" and "empowerment" (Dwyer 1993). The operation of power itself is often masked by the way a particular discourse is constructed (see further discussion of this point in 3.3.2). As Fairclough points out, the most effective wielding of power is often not 
openly forceful, but instead gains the collusion of those who are subject to it (1992b:50). Repressive discourse is one example of this, where the language forms used are superficially friendly and polite, but the underlying message is a manipulative one, intent on getting the addressee(s) to conform or agree to do something which they do not necessarily wish to do.

By contrast with CA and other micro-analytic approaches, the wider context is vitally important in critical discourse analysis. Fairclough suggests that any discursive event "is seen as being simultaneously a piece of text, an instance of discursive practice, and an instance of social practice" (1992a:4). In the field of workplace communication research, adopting this "three dimensional framework" means the analysis of communication and discursive practices within institutions cannot be separated from a contextual analysis of the institutions themselves (Candlin 1987; Mumby and Clair 1997), and implies a need for "intertextual" and "interdiscursive" analysis (Bakhtin 1986; Fairclough 1993, 1995).

\section{Discourse and social identity theory}

Theories of discourse and social identity intersect with CDA, and likewise operate within a critical social constructionist framework, viewing language as a dynamic process, "a set of strategies for negotiating the social landscape" (Crawford 1995:17). Language is viewed as the site of the cultural production of social identity: subjectivity is thus discursively constituted (Weedon 1987; Butler 1990). In other words, each person's identity is continually constructed (ethnicised, gendered, professionalised and so on), within the social, economic and political discourse to which they are exposed (Weedon 1987:21). In the words of Iedema and Scheeres:

... (f)or discourse analysts, this means that we are engaging with language not as objective and finite instrument, but as socio-political and strategic means to enacting and (re)negotiating identity and positioning. As workers shift from doing to talking work, the work of discourse analysts is shifting from examining 'language in a practical context', to engaging with enactments and management of workplace self, manifested through language as well as other semiotic modes. (Iedema and Scheeres 2003:335)

According to this approach then, people operate within subject positions created and sustained by the use of language. Speakers are regarded as constantly "doing" identity work, and the different ways in which people behave are accounted for by the 
sociocultural contexts in which they operate. Within this paradigm, identity, in all its aspects, is not regarded as given, but rather, is problematised and examined as a social construction with discourse playing a crucial role in this process.

\subsubsection{Organisational theory}

Organisational theory provides a contrasting perspective on workplace (mis)communication and discourse to the other theoretical and methodological paradigms discussed so far. Relevant areas of inquiry briefly canvassed in this section include both traditional and more recent discursive approaches to organisational communication, systemic approaches (in particular systems and human factors approaches to error, safety and quality assurance), and genre and activity theories.

\section{Organisational communication}

Organisational communication is an interdisciplinary field with its primary roots in organisational behaviour, management and communication studies, but it also draws on a wide range of approaches in the social sciences and humanities (Jones and Stubbe 2004). Putnam (2000:225) identifies "the reflective, the constitutive, and the equivalent positions" as three contrasting perspectives commonly found in organisational discourse analysis. In the traditional "reflective" approach, organisations are seen as "containers" where language represents and reflects organisational structures and processes. This view is increasingly being rejected according to Putnam, because it "trivialises language use, reifies the organisation, and pays little attention to the dynamics of organising" (ibid). The "reflective" approach underlies the instrumental, skills-based models of interpersonal communication in workplaces traditionally seen in this discipline, as well as in communication studies more generally (e.g. Adler et al 1996; Stewart et al 1997; Verderber et al 2009).

In recent years however, there has been a post-structuralist discursive turn in this field (e.g. Alvesson and Karreman 2000), as in many other communication-related and social disciplines. Whilst versions of the traditional positivist approach remain present in the literatures of management, organisational behaviour and communication studies, nowadays the field of organisational communication is more centrally defined by the proposition that communication is organisation (e.g. Taylor and Lerner 1996; Taylor et al 2001). Organisational theorists are thus increasingly 
likely to work within a social constructionist paradigm taking either a "constitutive" position, where language and organisations are considered to be in a dynamic interaction, co-producing one another (e.g. Pearce 1994), or an "equivalency" position where "organising becomes communication through the intersection of discourse and text" (Putnam 2000:225).

Researchers in this discipline rely mainly on more macro-level observational and reported data by comparison with the focus on analysing localised and naturallyoccurring interaction and intergroup dynamics in many of the broadly linguistic approaches discussed above. Nevertheless, the grounding of organisational communication research in the wider perspective of organisational studies makes it a particularly useful framework for looking at organisational issues in communication terms, not just at communication in workplaces (Jones and Stubbe 2004:193). This allows workplace practitioners and discourse analysts alike to see communication as more than just a set of personal skills or deficits (see also 3.2.2), and to move away from the notion of prescribed communication competencies towards a more flexible and complex approach to workplace communication and learning (Antonacopoulou and FitzGerald 1996; Musson and Cohen 1999).

There is also a growing trend for language and communication research in specific institutional and organisational domains but from different research traditions to be drawn together in productive cross-fertilisations which yield new theoretical insights and new avenues for applying these to professional practice. Examples include: health communication and discourse analytic research (Sarangi 2005); organisational communication and discourse analysis (Putnam 2000; Putnam and Fairhurst 2001); organisational communication and interactional sociolinguistics (Jones 2002; Jones and Stubbe 2004); health communication and conversation analysis (Drew et al 2001; Heritage 2007); organisational communication and conversation analysis (Tulin 1997).

\section{Systemic approaches}

There is a long tradition of applying systems models of various kinds to the study of organisations and organisational communication; such approaches are commonly applied, for instance, to process analysis and the tracking of networks and channels of information flow within organisations. Of particular relevance to the present study is the influence of different brands of systems theory on the study of 'human factors', 
including communication, in the ontogenesis of organisational error or adverse events. Arguably one of the most important developments within the field of organisational studies in the last two to three decades has been the development of a "whole systems" or ecological approach inspired by the work of Gregory Bateson amongst others (Hawkins 2004). Systemic approaches take an organisation-wide perspective on institutional processes, including different aspects of communication, in which particular and/or localised phenomena are seen in the context of the system as an integrated whole (e.g. Argyris and Schon 1995; Gatenby and Jones 1995; Sligo and Bathurst 2005). In the words of management theorist Peter Senge:

... (t)he discipline of systems thinking lies in a shift of mind: seeing interrelationships rather than linear cause and effect chains; and seeing processes of change rather than snapshots. (Senge 1990, cited in Hawkins 2004:417)

Such models set out to examine the interrelationships between people, the tools they use and the environments in which they work with the overall aim of improving systems and processes (Weinger et al 1998).

A more recent development in the systemic approach is the emergence of complexity theory as a framework for studying organisations and organisational communication, as part of a wider "complexity turn" in the social and cultural sciences (Urry 2005). Complex systems are, by definition, open, non-linear, dynamic, emergent and selforganising, changing over time as a function of interaction among their components and with the environment (Richardson 2005; Urry 2005; Cameron and LarsenFreeman 2007; Sturmberg and Cilliers 2009). Within this paradigm, an organisation or work team may be seen as a complex adaptive system - "an intricate, complicated network of independent agents that are so interconnected as to form a unity or organic whole with the capacity to adjust to changing circumstances and to anticipate future events" (Wheelan and Williams 2003:445). Specific characteristics such as patterns of communication are seen as emergent properties of such complex systems. Indeed, the argument has also been put forward in a recent book which relates complexity theory to the practice of applied linguistics, that language-in-use can itself productively be viewed as a complex adaptive system (Cameron and LarsenFreeman 2007; Larsen-Freeman and Cameron 2008).

Complexity theory is also being applied in research into specific aspects of organisational communication such as information systems and teamwork. Thus 
Heyligen (2008) discusses the science of self-organising systems and complexity theory as a "powerful new perspective" for understanding how virtual information systems work, while groups and teams have also been characterised as complex adaptive systems (e.g. Wheelan and Williams 2003; Gilstrap 2005). Interprofessional team communication is described as "a shared and distributed work activity ... that may be constructed through intentionally collaborative practice or (as) an emerging property of a complex adaptive system" (Bleakley 2006:305). The application of complexity science to interprofessional teamwork, communication and training in medical settings is also a strongly emerging theme in the current patient safety literature (e.g. McKeon et al 2006; Evans 2007; Woods 2007). Other researchers have also commented on the usefulness of understanding the medical consultation itself and/or patients' ongoing relationship with their own doctor (e.g. Innes et al 2005; Sturmberg and Cilliers 2009), and/or interactions with the health system throughout an episode of care (Stubbe, Dew et al 2009), as other examples of complex adaptive systems, due to the inherently uncertain and non-linear trajectories of such interactions.

\section{Genre and activity theories}

Activity- and genre-based theories represent another distinct and important set of approaches to the study of discourse in organisational settings (e.g. Freedman and Medway 1994; Bargiela-Chiappini and Harris 1997b; Christie and Martin 2000; Bazerman and Russell 2003) and in this context are closely related to the systemic thinking perspective outlined above. In activity theory, the meanings of human artefacts, which include written, spoken and electronic texts of various kinds, can only be understood in relation to and within the context of the activities that give rise to them (Bazerman and Russell 2003:1). Whilst traditionally focussing primarily on the description of institutionalised written texts, genre analysis has become more multi-faceted (Orlikowski and Yates 1994; Bhatia 2002), and is also sometimes applied to spoken genres, and to the interactions between sets of written, electronic and spoken genres and various mediating technologies in a range of organisational and institutional settings (e.g. Smart 2003; Schryer et al 2003; Schryer et al 2007). Within this paradigm, the different genres used in organisations are seen as a type of "socio-rhetorical action" (Smart 2003:14) and typically function together in sets. These sets of genres can in their turn be viewed as one part of a distributed "activity 
system" oriented towards accomplishing communally defined goals (Cole and Engestrom 1993; Engestrom et al 2003). Taken together, these genre sets form a "discursive fabric of temporally and functionally linked written texts and spoken performances" (Smart 2003:22).

Drawing on theories of "situated learning" (Lave and Wenger 1991; Wenger and Snyder 2000), Smart (2003) defines an organisational activity system as "people collaborating - over time - within an organizational community of practice - in goaldirected activity - using culturally constructed tools to think and act with" (16). Here the term 'community of practice' refers to any group of people participating in an (organisational) activity system, which is a somewhat broader definition of this concept than that proposed either by Lave and Wenger, by sociolinguists such as Eckert and McConnell-Ginet (see 2.3.3 above), or by some other discourse analysts working in the area of professional discourses (e.g. Sarangi 2002).

The concept of the "genre system" or "genre network" (Bazerman 1994) relies on the Bakhtinian notion of intertextuality (Bakhtin 1981, 1986; Swales 2004). This concept is key to the interest of genre-based theories in the interactivity of different communicative episodes and overlapping activity systems within a particular community of practice (Bazerman 1994; Timmermans and Berg 2003). Because the focus is on "interrelated systems that connect the past to the future" (Schryer et al 2003:70), ethnographic, phenomenological and interpretive methodologies are the mainstays of organisational genre research. The aim is to explore the discursive and conceptual "lived reality" of organisational members to produce a "thick description" of their communicative practices (Geertz 1973, 1983; Sarangi and Roberts 1999a). For example, such an analysis might explore how a genre system allows for the discursive management of activities such as troubleshooting a workplace problem or negotiating roles and identities, and/or the ways in which those in control of the system can manipulate, deliberately or simply out of routine practice, what information is selected, what continues to be attended to (or is dropped) and how information and relationships get (re)interpreted over time. 


\subsection{Summary and discussion}

As we have seen, existing research in the domain of workplace communication draws on a wide range of epistemological and methodological positions, both theoretical and applied, and is often located within an explicitly interdisciplinary space. This chapter has provided something of a whistle-stop tour of the main theoretical frameworks and methodological approaches commonly applied to the analysis of spoken discourse in workplace settings by researchers from different disciplines, including linguistics, sociolinguistics, psychology, sociology and organisational studies. The six broad analytic paradigms considered and briefly reviewed here were conversation analysis, pragmatics and politeness theory, sociolinguistics (including the ethnography of communication, interactional sociolinguistics and communities of practice), social psychology (including communication accommodation theory and discursive psychology), critical discourse theory (including CDA and social identity theory) and organisational theory (including organisational communication, systemic approaches and genre/activity theories).

The analytic focus of these approaches covers a similarly broad spectrum. Discourse analysis methodologies can be roughly classified into two major types (see also 2.2.2 and note 2). These reflect the researchers' primary interest in either the 'micro' or the 'macro' levels of analysis respectively, and also reflect the degree to which their analyses account for (or are embedded within) the local and/or wider interactional and social context. In workplace communication research, this often translates into a distinction between studies which focus primarily on the negotiation of meaning and communicative processes in task-oriented interpersonal or intergroup interactions in specific settings, and those which focus on the contextualisation of talk in widerranging social and power relationships or organisational systems.

Styles of discourse analysis with a small 'd' (e.g. conversation analysis, pragmatics, interactional sociolinguistics) have as their primary concern the fine-grained detail of single interactions, and tend to be predominantly descriptive in nature, although they can and often do include critical and/or applied perspectives as well. Discourse analysis with a big ' $\mathrm{D}$ ' looks at the big picture, often taking an explicitly ideological or critical perspective on the interdiscursive construction and maintenance of institutional orders (e.g. CDA), or else a wider systems or organisational perspective. 
A further distinction can be drawn between approaches where language or discourse in a particular workplace setting is itself the primary target of analysis, and approaches where the analytic focus is primarily on a practical or organisational issue of some kind, for example a logistical, relational or intergroup problem, which may be mediated by communication, but is distinct from it.

A number of researchers working in the field of workplace/institutional communication take a third way by integrating close textual analysis with a more macro-level and/or critical discursive approach in an attempt to "explain why communicative behaviour varies according to the specific structural conditions in which it takes place" (Wodak 1996:7). This applies particularly to certain work based in interactional sociolinguistics (see for example: Gumperz 1992, 1999; Drew and Heritage 1992a; Roberts et al 1992; Sarangi and Roberts 1999a; Holmes and Stubbe 2003a; Roberts and Sarangi 2005; Dew, Plumridge et al 2008); in applied conversation analysis (e.g. West and Frankel 1991; Kitzinger 2005b; Stivers and Majid 2007; Heritage 2008); and within a critical theory/organisational paradigm (e.g. Fairclough 1989, 1995; Wodak 1996; Mumby and Clair 1997; Swales 2004; Rouveyrol et al 2005; Baxter 2008).

The models and analytic approaches which have been summarised in this chapter are those principally drawn on in the discussion and analysis of miscommunication and problematic talk which follows in subsequent chapters. The next chapter turns to a more specific consideration of the research literature which deals with miscommunication and problematic talk at work. This will include a summary and discussion of relevant definitions and models of miscommunicative talk in the existing literature, followed by an overview of applied research which has focussed on these issues in a range of workplace settings and at different levels of analysis. 


\title{
3 Literature review (b) Miscommunication and problematic talk
}

\begin{abstract}
Language is deceptive; and though English is subtle it also allows a clever person - one alert to the ambiguities of English - to play tricks with mock precision and to combine vagueness with politeness. English is perfect for diplomats and lovers.
\end{abstract}

Paul Theroux, The London Embassy

\subsection{Introduction}

Miscommunication has been called "an interesting and slippery concept" (Coupland, Wiemann and Giles 1991:11). This chapter provides an overview of the various ways this concept has been defined and understood in the existing literature and the kinds of findings generated using these models. The main focus is on research into problematic spoken discourse in institutional and organisational settings, with selective reference to several significant bodies of research in which problematic communication is an important theme, but where the primary focus and/or domain of inquiry is distinct from and/or beyond the scope of the central concerns of this thesis. This latter category includes work in the wider fields of intercultural communication, language and gender, organisational communication, and human/systems error research.

There is a long history of research on miscommunication and 'problems of understanding' in spoken language. The relevant literature spans several decades, is located within various theoretical paradigms and disciplinary domains, and focuses more or less widely or narrowly on different aspects, levels and social contexts of communication. This diversity of perspectives and research traditions poses a considerable challenge to any researcher who is interested in forming an integrated perspective on these phenomena, as pointed out in the seminal volume Miscommunication and Problematic Talk (Coupland, Giles and Wiemann 1991). In the editorial introduction to that volume, Coupland, Wiemann and Giles present a preliminary typology in the form of an "integrative model of levels of analysis of 
'miscommunication"” (1991:16), as a first step towards achieving a more integrated perspective (see 3.2.2 below). But almost two decades on, we still lack the comprehensive theoretical model called for by Coupland, Wiemann and Giles. Arguably the main reason for this continued lack of coherence in the research literature is the fact that problematic communication is such a ubiquitous, multifaceted and highly contingent phenomenon that it continues to resist straightforward classification or definition.

The next section (3.2) reviews the key conceptualisations of miscommunication and problematic talk as they are defined and understood in the existing linguistics, discourse studies and organisational communication literatures. This is followed in section 3.3 by a selective overview of previous research into miscommunication and problematic talk relating specifically to workplace settings. The final section summarises the gaps and limitations identified in the existing research as pertaining to the aims of the present research, and signals some potential avenues for more adequately addressing the theoretical and methodological issues identified, before setting out the specific research questions which will be the focus of the remaining chapters.

\subsection{Existing models of miscommunicative talk}

Previous research on miscommunicative talk incorporates a number of different perspectives on what counts as miscommunication (i.e. the definitional criteria and categories used), and why, how and where it occurs (i.e. the ways in which its underlying causes are modelled). The discussion below takes as its starting point the heuristic template or "integrative model" proposed by Coupland, Wiemann and Giles (1991) which is based on a "structural, layered organization of perspectives on miscommunication" grouped according to their underlying analytic goals and assumptions (12).

\subsubsection{Some problems of definition}

The first issue that needs to be dealt with in any conceptual model is the delineation of the phenomenon of interest. The meaning of 'miscommunication' is often taken to be self-evident, but a review of the existing literature makes it very clear that defining precisely what 'counts' as miscommunication or problematic discourse is no 
straightforward task, and many related analytic issues of identification, classification and interpretation remain unresolved. ${ }^{1}$ This is seen most obviously in the difficulties that arise when we try to deconstruct exactly what the terminology used by different researchers actually refers to, and what 'models' of communication are being invoked.

In the first instance, as Coupland, Wiemann and Giles (1991) observe, the use of the word 'miscommunication' itself is highly problematic: it is often applied very loosely to any interactional problem that arises (see also chapter 1), or, conversely, quite narrowly to very localised processes of 'misunderstanding', regardless of the degree of 'severity' of the outcome, as in much of the pragmatics literature. Both usages fail to capture the full diversity and complexity of the phenomena we are interested in here, as will be amply be demonstrated below and in chapters 5 to 7 . Coupland, Wiemann and Giles (1991) themselves use the umbrella phrase "'miscommunication" and problematic talk" (also adopted in this thesis) to encompass in abstract terms the full range of phenomena they wish to consider. In their review of the extant literature in 1991 they talk of miscommunication in this broader sense as being "operationalised" (7), or "surfacing" as "misunderstandings" (4), "mismatches" (5), "miscommunicative sequences" (6) "communication failure" or "inadequacy" (8), "breakdown" (9) or "misalignment" (10), and comment that "(i)n fact, it is rarely possible to operationalize miscommunication purely at the propositional level, except perhaps in circumstances ... in which relationships and affect are not a primary issue" (7) .

Other researchers have similarly recognised the analytic importance and usefulness of drawing a distinction in the terminology they use between the overall phenomenon of 'miscommunication' and individual instances. Localised occurrences have been labelled variously as "misunderstandings" (e.g. Bazzanella and Damiano 1999; Tzanne 2000), “mismatches" (e.g. Morgan 2008), “interactional "trouble” (e.g. Tracy 1997) or "dilemmas" (e.g. Heritage and Sefi 1992; Gill and Maynard 2006) and "miscommunication events" (Linell 1995), amongst others. On the other hand, in much pragmatics writing on this topic, the distinctions are made at a much finer level of detail. For example, Dascal (1999) positions misunderstandings as taking place at

\footnotetext{
${ }^{1}$ These issues of definition will be more fully explored as part of the illustrative analyses to be presented in chapter 5 .
} 
the level of a single turn or exchange, while miscommunication is defined as a misunderstanding that persists for several turns (Dascal 1999), or as an unresolved breakdown in communication caused by sustained misunderstanding across a longer interactional sequence (Weigand 1999).

The term 'problematic talk' is also used in different ways: sometimes as a superordinate or umbrella term, or alongside other descriptors such as miscommunication, misunderstanding or communication breakdown as a way of drawing a particular distinction. As we have seen, Coupland, Wiemann and Giles (1991) use this term in conjunction with 'miscommunication' to encompass the phenomenon as a whole. Aronsson (1991) in the same volume defines miscommunication as talk which leaves unintentional discrepancies between participants in their interpretation or understanding of what has been said or done. Linell (1995) suggests 'miscommunication' should be categorised as a subset of problematic talk, and follows Aronsson (1991) in excluding deliberate discrepancies such as lies, deliberate equivocation or misleading, evasiveness or strategic silence. This latter definition of miscommunication is located very much at the local interactional level, and would include "non-deliberate unclarity of expression, misrepresentations, mishearings, misunderstandings, misconstruals of others' utterances, misquotes, misattributions (of intentions to interlocutors), talk at crosspurposes, mismatches of interactional and topical coordination etc." (177).

This diverse set of descriptive terms reflects a similar diversity in conceptualisations of what the phenomenon of miscommunication actually is, and in the methodological frameworks within which it has been researched. One consequence is that similar terms are often applied to quite different kinds of phenomena at different levels of analysis, or conversely, different terms may be used by different researchers to refer to the same or similar phenomena. At the same time, the criteria for identifying or classifying something as an 'instance' of miscommunication are also rather inconsistent (see below). Such ambiguities and lack of consistency in the literature are confusing in their own right, but these difficulties are themselves diagnostic of the 'slippery' nature of the underlying concept of miscommunication itself.

Most fundamentally, terms like 'miscommunication', 'communication breakdown' and 'communication failure' beg the question of what is 'good' and what is 'bad' by automatically framing 'miscommunication' as a deficiency that should (and can) be 
avoided or repaired. Coupland, Wiemann and Giles (1991) make the case that all communication is intrinsically an imperfect process: “... language use and communication are in fact pervasively and intrinsically flawed, partial and problematic", and that communication is thus "to that extent ... itself miscommunicative" (1991:3). They point out that even in discourse contexts where we can assume the existence of mutual goodwill and a desire to achieve effective communication on the part of participants, some degree of 'slippage' is almost inevitable (1991:11). Because meaning resides in the interaction of linguistic form and social context, utterances are in themselves intrinsically indeterminate and open to multiple interpretations depending upon the presuppositions and goals of participants (van Dijk 1987; Linell 1995) and/or on how their meanings are coconstructed as an interaction unfolds (ten Have 2007). In other words, "language does not 'convey' meaning; it simply helps to produce it" (Lee 1992:191). Because “(m)iscommunication cohabits with communication in dialogue" (Linell 1995:184), it should thus be seen as a normal and ubiquitous part of human interaction, rather than as an abnormal deviation from it (Sperber and Wilson 1985; Coupland, Wiemann and Giles 1991; Fraser 1993; Dascal 1999; Anolli et al 2002; Bou-Franch 2002).

Secondly, we cannot necessarily assume that interlocutors intend to be clear, or that they do in fact have shared goals. In many cases, a particular interaction, or a more extended discourse sequence, will be characterised by multiple and/or divergent goals. Apparently problematic talk can also fulfil positive social functions. For instance, ambiguity, indirectness or the selective imparting of information may better accomplish the relational or political ends of one or more parties than completely clear and honest communication would (Eisenberg and Phillips 1991:248-9), or it may function either deliberately or unintentionally as a face saving device (Tzanne 2000:223). Linell (1995:185) suggests that some episodes of misunderstanding may even "increase the depth of understanding in ways that, without them, would be difficult to come by." Apparent or even actual 'miscommunication' then, can promote desirable outcomes, and it is to be expected that people will often be indirect or even "sceptical, crafty and less than veracious" (Giles and Wiemann 1987). Conversely, clear, concise and 'honest' communication may also cause difficulties, in organisations as in private interactions, as often as it is the solution to them (e.g. 
Redding 1972), and in any case does not often reflect the reality of everyday workplace interaction (Holmes and Stubbe 2003a).

To further complicate matters, (as also observed in chapter 1), talk can be "deceptively adequate"; that is, it may appear superficially to be unproblematic, whilst some degree of miscommunication in fact exists at a deeper level (Coupland, Wiemann and Giles 1991:7), thus creating "an illusion of understanding" which may or may not ever surface or be resolved (Roberts 2009:113), or which may be "treated" without being resolved by way of a "pseudo-solution" (Linell 1995:189). In short, deciding whether a given instance of communication should be assessed as 'effective' or 'problematic' is highly context-dependent and also likely to vary according to the perspective from which it is viewed (Carney 1993). Being maximally clear and unambiguous in interpersonal interactions may be 'good communication' at one level, but it may equally be 'miscommunicative' at a number of others.

If we accept these premises, then it is clear by extension that miscommunication and problematic talk are not in binary opposition to or a deviation from some norm of 'effective' or 'unproblematic' communication - however these constructs might be defined in their turn. Rather, as several researchers in this area have remarked, miscommunicative and problematic talk are part and parcel of the rich fabric or continuum of human language and interaction (cf. Sperber and Wilson 1985; McTear and King 1991; Anolli et al 2002), whether this takes place at work or anywhere else. As so neatly identified in the quotation from Theroux at the opening of this chapter, there is an inherent paradox at work here: namely, the richness and complexity of language makes it a powerful strategic tool for social and 'political' interaction, but this very fluidity can also make communication problematic, and it certainly renders miscommunication a very difficult concept to define and operationalise analytically.

Difficult as it is to define precisely what constitutes miscommunication and problematic talk, this represents only one part of the task at hand. Identifying precisely where these phenomena are located when they do occur, on what basis they should be recognised as such, and classifying them according to why and how they come about are equally complex and problematic issues. The answers to these kinds of questions are ultimately a product of the focus of inquiry in a given case and of the epistemological paradigm within which it is framed. In other words, which 
dimensions of miscommunication are being investigated at what analytic level, and what basic model of communication (see chapter 2) is being assumed?

Depending on the underlying conceptualisation, miscommunication and problematic talk will be identified at different levels of discourse. At the level of pragmatics or talk-in-interaction, miscommunication is seen to occur at either or both the referential/propositional and affective/social/relational levels of language use. It may be viewed as a product of speaker-based mis(re)presentations or hearer-based misunderstandings (Bell 1991), or as one result of interactional 'trouble' with the coconstructed negotiation of meaning (e.g. Jefferson 1984, 1988; Schegloff 1987; Tracy 1997). Alternatively or as well, problematic talk may be seen to arise from systematic socio-cultural differences or patterns (e.g. Gumperz 1979). It may be characterised as a localised, discrete event embedded in a linear process (e.g. Tzanne 2000), as a "global" miscommunication "characteristic of a whole interaction or a phase of its discourse" (Linell 1995:190), or as part of a more complex set of interactional dialectics embedded in the intertextual or "discursive fabric" of institutions and other social structures (e.g. Smart 2003). In some models, miscommunication arises out of (and/or helps to construct and maintain) inherently problematic and largely invisible aspects of social or institutional structure (e.g. McTear and King 1991; Wodak 1996).

In addition, there are matters of 'agency', perspective, awareness/recognition and repair or outcome to consider, and there is little consistency in the literature as to whether a particular phenomenon should be considered as an instance of miscommunication or not depending on how variable factors such as these are interpreted. First of all, miscommunication may be viewed as being accidental or unintentional, the product of imperfect processes or a lack of individual competency (cf. Aronsson 1991; Linell 1995, as discussed above), or it may be considered to entail a degree of intentionality or even a "moral dimension" (Coupland, Wiemann and Giles 1991:10). Secondly, as already noted above, something that is miscommunicative from one point of view may also represent a perfectly desirable outcome from another perspective. Finally, participants might be fully aware of an actual or potential problem and will successfully avoid or repair it, but it is equally possible for a misunderstanding to pass unnoticed by the people involved, even if it is observable by others, or if its consequences become apparent subsequently (Linell 
1995). As will be discussed further in chapter 5, it is far from clear where we should draw the line analytically in each of these cases.

Unsurprisingly therefore, analysts from different traditions routinely disagree on what counts as evidence that an 'instance' of miscommunication has occurred, and thus what counts as miscommunication in the first place. Conversation analysts for example consider that "analysts should not discuss stretches of talk as involving misunderstandings unless participants themselves have treated them as such", as evidenced by an observable orientation or action in the interaction itself (Schegloff 1984; Bilmes 1992). As we saw in chapter 2, researchers from other traditions who identify miscommunication in different terms will admit different kinds of contextual information or interpretive frameworks to the analysis. The ways in which these constructs are conceptualised and defined inevitably determines what kinds of data are examined, which 'instances' of miscommunication are identified and how these are categorised and analysed. At the very least it needs to be recognised that miscommunication and problematic talk are 'relative' and not 'absolute' concepts which inevitably makes them something of a moving target from a theoretical and analytic perspective.

\subsubsection{An "integrative" model?}

Given the complexity of the phenomenon of miscommunication, and the diverse and sometimes cross-cutting nature of existing conceptualisations, the development of a more integrated framework would indeed seem to be useful. To date, the "integrative model of levels of analysis of 'miscommunication'" proposed by Coupland, Wiemann and Giles (1991:13), is still the best attempt on offer to tease out some of these complexities. However, it must be noted that this model came with the caveat that it should be seen as "a preliminary template against which researchers ... may locate their own perspectives on miscommunication, and consider others"; as such, it was intended to serve a primarily heuristic function, rather than claiming to be a comprehensive explanatory model in its own right (16). Although this template therefore has a number of limitations and does not in itself provide a comprehensive theoretical model (see below), it nonetheless provides a useful starting point for mapping out the analytic landscape in this area in greater detail. 
Coupland, Wiemann and Giles' (1991) "integrative model" is organised into six "levels" which represent a spectrum of 'problem' categories or characteristics of miscommunication. These levels are primarily intended to reflect the basic assumptions made in different (sets of) research paradigms about where miscommunication is located, along a proposed continuum of progressively 'deeper' analyses and 'weightiness' or social significance. At the same time, the template also attempts to capture differences between the levels in 'repairability', and how aware or otherwise participants or analysts are likely to be that a problem exists. The main characteristics attributed to miscommunication by Coupland, Wiemann and Giles, and my own summary of and commentary on the main theoretical approaches to miscommunication at each of the six levels are briefly summarised below. ${ }^{2}$

Levels 1-4: Localised disruptions, deficiencies and goal conflict

The first level in this model is based on the premise discussed above that all communication is to some extent intrinsically imperfect or problematic; although miscommunication can technically be said to occur at this level as part of an ongoing negotiation of meaning, in most cases it would not be labelled as such, and the issue of repair therefore does not arise. At level two, communicative efficacy starts to be affected by usually quite minor 'mishaps' of understanding or deviations from an assumed 'norm' which may or may not be recognised as miscommunicative and hence repairable by participants (see also chapter 2.3.2). This is the level at which most pragmatics studies of 'misunderstanding' are positioned, where in the "standard case" (Weigand 1999) most misunderstandings are detected in the immediately following turn and successfully repaired by the third or fourth turn (Bazzanella and Damiano 1999). Level three is associated with deficit or competency models, often based in communication studies and applied linguistics (see also chapter 2.3.6), which assume miscommunication arises from individual differences or gaps in social or communicative skills, personal styles or attitudes that in workplace settings can be 'fixed' via training or the implementation of protocols and assessment tools (cf. Antonacopoulou and Fitzgerald 1996; Tourish and O'Hargie 2000; Reason 2000; Jones 2002).

\footnotetext{
${ }^{2}$ Levels 1-4 in the original model have been subsumed under a single heading for my purposes here, and the descriptors for the levels are my own paraphrases of the original wordings provided by Coupland, Wiemann and Giles (1991).
} 
Models categorised at levels one to three are all predicated on the assumption that miscommunication is a largely unintentional by-product of some kind of inherent deficiency in the channel, process or 'system' of communication, or the result of a knowledge or 'competency' deficit in one or more of the individuals involved. At these levels, miscommunication may be analysed as failed understanding or transmission of information, or as a failure to achieve desired outcomes in terms of quite specific and localised interactional goals (Eisenberg and Phillips 1991). These goals may include the achievement of task-related or relational outcomes (Coupland, Wiemann and Giles 1991:14). In other words, communication problems might occur because the propositional content of a message or discourse sequence is unintentionally misrepresented or misunderstood, and/or because the relationship between speaker and hearer is disrupted in some way (Bell 1991; Dascal 1999). Problems at these levels can include non-communication (where information is not passed on at all, or has gaps), inaccuracy, lack of clarity, distortion, misreporting or misattribution.

Linear sender-receiver (or transmission) models of communication would typically fit at these levels, as do, in part, some other psychological models discussed in chapter 2 such as those based in classical pragmatics and sociolinguistics (e.g. Grimshaw 1980; Tannen 1981; Bavelas 1985; Perkins 1998; Dascal 1999), and many of the mainstream communication studies and communication error models (e.g. Stewart 1997; Reason 2000; Verderber et al 2009; Trenholm 2010) discussed in 2.3.6. Models of miscommunication located at these levels can be useful in clearly defined or routinised contexts where the main source of the problem lies in the articulation or transmission of referential or information content and/or where there are minor and clearly identifiable 'normative' infelicities at the pragmatic level. In practice however, the largely descriptivist accounts provided by such models often fail to account adequately for contextual or systemic factors, or for the dynamic interactional dimensions of more complex instances of miscommunication and problematic talk (see also 3.3 and chapter 5 below).

At level four, "strategic use of communication enters into the analysis" (Coupland, Wiemann and Giles 1991:14). Here miscommunication is seen to arise from a failure to negotiate a "working consensus" or to achieve interactional alignment (as well as or instead of the issues noted above), and may include a greater or lesser degree of 
intentionality. An underlying assumption of models positioned in this category is that interactants normally orient to multiple, simultaneous (and sometimes conflicting) goals. Interactions therefore are assumed never to take place purely at the propositional or instrumental level, and may simultaneously include affective, relational and identity management dimensions (cf. Coates 1987; Stubbe and Holmes 1995; Heritage 2006). This set of constructs underlies much research relating to face work, indirectness, vagueness and ambiguity in language use, and is often invoked to explain why what appears on the surface to be the most economical or efficient communication strategy (e.g. being direct and explicit, or following a strict protocol) may not always be the most effective one and/or what 'members' can be observed to do in actual practice (e.g. Holmes and Stubbe 2003a; Dew, Stubbe et al 2010). Relevant frameworks here include pragmatics and politeness theory (e.g. Brown and Levinson 1978, 1987; see also 2.3.2), and interactional sociolinguistics, conversation analysis and ethnomethodological approaches (e.g. Gumperz 1982a, 1982b; Bilmes 1992; Harris 2003; Boden 1994; see also 2.3.1 and 2.3.3).

Communication breakdowns at this level may also be analysed as a misreading of another's intentions or attitudes, often as a result of conflicting "metamessages" or incorrect inferencing based on divergent expectations, implicit assumptions or attitudes (e.g. Tannen 1990; Bing 1997). As Erving Goffman has argued, the set of assumptions associated with a particular event or situation acts as a filter or lens by means of which we sift our general stock of knowledge in order to retrieve what we need to know for the purposes of a given encounter (Gumperz 1992:307). The working assumption in framing theory then is that people structure experience and interpret the meaning of utterances through different "interpretive frames" (Bateson 1972; Goffman 1974), and that where these conflict, miscommunication is likely to occur. Framing theory (e.g. Goffman 1974; Tracy and Coupland 1990; Gumperz 1992; Tannen 1993a) can therefore fit at this level as well as at level five (see below).

Models positioned at level four provide some very powerful tools and theoretical concepts for the analysis of miscommunication and problematic talk, especially where a critical lens is also added to the analysis. As we will see in the next section, researchers using such approaches have generated a wealth of useful and interesting findings which are often directly relevant and applicable to communication issues in 
workplace settings. However, as discussed in the previous chapter, even though aspects of the immediate context and socio-cultural frames are usually taken into account, analysis of miscommunicative talk using these kinds of approaches does not generally extend beyond the boundaries of a single interaction. Because much workplace communication is strongly intertextual and consists of related episodes in an ongoing dialogue rather than a series of independent interactions, this represents a significant limitation on the analysis of miscommunication in this setting (see chapters 4 and 5). Some fundamental theoretical differences can also make existing research using these different approaches difficult to reconcile (cf. Stubbe et al 2003). In particular there are differing epistemological stances on whether conversational goals and frames are considered to precede their articulation in language, or to be co-constructed in the course of an interaction, and with regard to the place of contextual information and sequentiality in the analysis of localised misunderstandings.

\section{Level 5: Differing group norms and discursive practices}

Level five relates to miscommunication arising primarily from differences in intergroup or cultural norms. It is usually assumed that this kind of miscommunication is unintentional and 'repairable' (or at least preventable) by means of learning, though here, unlike in the 'deficit models' mentioned above, it is learning about social processes rather than skills acquisition that is the focus. In cross-cultural contexts, it has been well-documented that members of different groups often "talk past each other" (Metge and Kinloch 1984:10); this is attributed to differences in their respective communicative styles, which in turn arise out of differing cultural and linguistic norms and practices (e.g. Gumperz et al 1979; Gudykunst et al 1996; Scollon and Wong-Scollon 2001; House and Rehbein 2004). Where a person interprets another's (linguistic) behaviour purely by means of their own cultural norms and expectations, they may interpret meanings that were never intended by their interlocutor. Conversely, they may fail to perceive signals that are present (Roberts et al 1992:208; Tzanne 2000). The results can range from irritation or confusion through to complete misunderstanding, but because participants interpret what is being said through different interpretive frames (see above), the possibility of different meanings is likely to go unrecognised, and may be incorrectly ascribed instead to interpersonal or intergroup differences in attitude, to personal characteristics or intentions, and/or to power or role asymmetries. This can easily 
lead to the establishment or reinforcement of negative attitudes, and thus to further miscommunication in a kind of vicious cycle (Stubbe 1998c).

Different group patterns or modes of interaction (i.e. communicative styles) have been reported to cause information loss and/or to help create negative attitudes towards members of another group in other contexts too, such as the different professional groupings or 'sub-cultures' within organisations (e.g. Kreitner and Kinicki 1992; Wilson 2000; Tietze et al 2003). Language and gender research is another example of a field where the 'two cultures' approach has been applied (e.g. Maltz and Borker 1982; Tannen 1993b, 1999; Coates 1993, 2004; Eckert and McConnell-Ginet 2003), though not without some controversy due to a rejection of the essentialist assumptions which often underlie cultural difference models of intergroup miscommunication (e.g. Henley and Kramarae 1991; Freed 1992; Cameron 1995; Stubbe et al 2001).

Once again, research predicated on models located at this level of Coupland, Wiemann and Giles' template has been very productive and often delivers results which are seen to be of immediate practical relevance in multi-cultural workplace settings, particularly when combined with other levels of analysis. For instance, social psychologists point out that it is important to recognise that intergroup tensions generally have their source in particular interpersonal or institutional interactions where group identity has some salience. As Gallois and Giles (1997:5) point out, "any interaction can be characterised along both interpersonal and intergroup dimensions", and even in interactions where the relationship between people is mainly interpersonal, group identities are never completely absent. In many interpersonal contexts it is quite common, for example, for individuals to be regarded as representatives of a group rather than as individuals (Tajfel and Turner 1979). 'Ineffective' communication skills and processes are therefore likely to compound any existing problems between groups, and models which seek to account for difficulties with intergroup communication therefore usually also operate at one or more of levels two to four and six as well.

In cultural difference models it is assumed that effective communication is most likely to occur where interlocutors either share the same vocabulary, interactional style and presuppositions (Roberts et al 1992; Ng and Bradac 1993), or else have a wide enough verbal repertoire (Schick Case 1995; Stubbe et al 2001) and are 
sufficiently flexible in using this, to adjust to the other's style in a process of mutual accommodation (e.g. Gallois and Giles 1998); the converse is true when these conditions are not met. It is usually claimed that communication problems caused by different group norms or world views are at once much more difficult to identify and to resolve than miscommunication arising from ineffective communication processes alone, as they have to do with underlying differences in assumptions and perceptions. Psychological/anthropological models of communication relating to social group membership (e.g. Tajfel 1982) and intercultural communication (e.g. Thomas 1983; Gumperz and Roberts 1991; Gallois and Giles 1998; Scollon and Wong-Scollon 2001; Spencer-Oatey and Jiang 2002) are grouped at this level (see also 2.3.3 and 2.3.4).

\section{Level 6: Competing and conflicting discourses}

The sixth and final level considered within the framework provided by Coupland, Wiemann and Giles (1991) is essentially ideological. Analysis of miscommunication at this level requires meta-analytic and 'critical' approaches because it tends to be associated with hegemonic structures which are largely invisible to participants (e.g. Kress and Hodge 1979; Fairclough 1989, 1992b, 1996; Henley and Kramarae 1991; Wodak 1996; Mumby and Clair 1997; van Dijk 1998; Weiss and Wodak 2003). Miscommunication here relates to social identities, value systems and structural power imbalances, which are especially salient in workplaces. These are seen as one typical site of "competing discourses" (Lee 1992) where meanings are not invariably shared and where relationships and interactions are seldom neutral in terms of power. As Lee (1992:189) points out, one consequence of linguistic indeterminacy at the utterance level is that "the meaning of a particular utterance is often open to negotiation and may be subject to "mystification"'. This may provide language users with a tool for "deleting or stressing particular aspects of reality" ( $\mathrm{Ng}$ and Bradac 1993:144), or with an alternative interpretive frame for understanding intergroup differences, thus encouraging the emergence of stereotypes. It is crucial therefore to analyse power relationships as part of any analysis of problematic institutional discourse (see also 2.3.5).

Analysis of problematic discourse at this level allows researchers to explicitly take account of the points made earlier (see 3.2.1), namely that miscommunication may be a deliberate strategy (where people appear to say one thing while actually saying 
something else), and that the perception of a given interaction or a more extended discourse sequence as 'well-managed' or 'mismanaged' may simply reflect participants' vested interests, where in some cases one or both parties may benefit from a continuing fracture (Candlin 1987:425). For example, critical discourse analysis permits a focus on how people manipulate the forms of language (e.g. vocabulary, or discourse structure and style) or selectively withhold information in order to mark a boundary between groups in order to 'do power', or analysis of the ways in which they may contest an issue or deny power to others through the prevention of discourse or the silencing of group members (Jaworski 1997). Discourse processes can thus be analysed as a means of masking or mystifying reality, and/or as a means of exclusion, and as a powerful tool for any individual or group concerned with shaping ideology or gaining power over others, something that has obvious relevance to problematic communication in organisational settings (see

\subsection{2 below).}

\section{Commentary}

The Coupland, Wiemann and Giles (1991) 'integrative levels' model provides a helpful taxonomy of the diverse theoretical perspectives on miscommunication and problematic talk found in the literature at the time it was constructed, and attempts to integrate these into a more unitary framework. This model identifies many important theoretical and analytic dimensions of these concepts, and succeeds in taking us beyond a single-perspective approach by highlighting where the points of convergence and divergence lie between different conceptualisations. It is certainly very useful as a heuristic framework for mapping out the theoretical terrain and for assessing the applicability of particular conceptual and analytic tools for different research purposes. Such a framework also provides an essential starting point for researchers who advocate invoking diverse theories and perspectives to achieve a more comprehensive account of particular instances or types of miscommunication by taking an eclectic "trans-disciplinary approach ... that transgresses traditional disciplinary boundaries" (House 2000). However, the extent to which this "preliminary template" (Coupland, Wiemann and Giles 1991:16) provides a robust basis for a truly "integrative" theoretical model of miscommunication and problematic talk, if this is in fact achievable at all, remains open to question. 
To begin with, it is arguable whether the heuristic framework of six 'high-level' categories or 'levels' represents a true continuum or hierarchy as Coupland, Wiemann and Giles seem to suggest. Firstly, whilst a distinction between more or less "superficial" and "deeper" levels of (mis)communication has some validity as a general theoretical principle, in practice, as we saw above, many existing 'models' or approaches can be analysed as operating at more than one such level at the same time, in recognition of the fact that a single perspective is often not enough on its own "to explain the richness and complexity of understanding and misunderstandings in discourse" (Bou-Franch 2002:19). As we also saw in chapter 2, discourse analysts often 'mix and match' analytic levels and frameworks in any case (more or less reflexively as the case may be) in order to satisfactorily account for what they have observed in their data. In other words, a given analytic model or framework does not necessarily slot neatly into one single position along this notional continuum.

In addition, any categorisation of existing conceptualisations of miscommunication along a uni-dimensional continuum is bound to be problematic, as there is always more than one way to 'slice' any complex phenomenon. For instance, as we saw in chapter 2, the various models in Coupland, Wiemann and Giles' six levels can also be mapped onto a micro - macro analytic continuum. They could also be classified according to whether they fit into a positivist or constructivist paradigm, or take a predominantly descriptive or critical approach. These distinctions all cut across one another in various ways, and as already discussed, different approaches which ostensibly sit at the same level in the Coupland, Wiemann and Giles' model may thus conflict with one another in other ways. The result is that each 'level' or category includes theoretical perspectives with many points of contrast as well as similarity, and as already mentioned, some approaches also span a number of levels at once. It therefore remains to be demonstrated whether such a classification of different approaches is more than just a convenient heuristic framework, or whether it also maps in some way onto a theoretically robust set of analytic categories.

Another limitation of the 'levels model' is that several analytic dimensions which are highly relevant to how miscommunication is conceptualised (see 3.2.1 above) are not integrated into the template in an analytically meaningful way. Some issues, such as 'problem status', degree of awareness, and repairability of miscommunication, are 
identified explicitly but are then simply cross-tabulated with the six 'levels' as variable factors. Others, including important and complex definitional and/or analytic issues such as perspective and agency, the types, causes and outcomes of miscommunication, and issues of context and intertextuality are mentioned but are not explicitly separated out and analysed further, which leaves their relationship with one another and the different levels of analysis unresolved. Whilst this does not necessarily matter for the purposes of a broadly descriptive characterisation of different conceptual frameworks, these factors do need to be thoroughly teased out and accounted for systematically as part of a comprehensive analytic framework.

A final limitation of the Coupland, Wiemann and Giles model (or a missing element, to be more precise), is simply a function of its age and historical context. Namely, recent complex systems approaches to problematic communication (see 2.3.6 and 3.3.3) are simply not considered at all as part of this model, and it is in fact difficult to see where and how this kind of conceptual framework would find a place in an integrative model of this type. Indeed, from a critical complexity theory perspective, it would not be seen as either helpful or possible to attempt to articulate the many different theoretical conceptualisations of miscommunication and problematic talk into a single and coherent 'integrated model' or paradigm (Richardson et al 2000; Grant 2003; Cilliers 2005; Richardson 2005, 2008). From this perspective, a multiparadigm, complex systems approach may therefore provide a more sustainable alternative framework for accommodating the diversity of perspectives and tricky analytic issues articulated in existing conceptualisations of miscommunication and problematic talk, a point which will be more fully elaborated in 3.4 below and in subsequent chapters. First however, a cross section of the most relevant existing research on problematic discourse in workplace settings will be reviewed to illustrate the range of communication issues documented in different domains of inquiry using the analytic models surveyed so far, and the kinds of strategies that have been suggested for 'managing' miscommunication and problematic talk at work.

\subsection{Miscommunication in the workplace}

Research into workplace (mis)communication is often interdisciplinary in nature, and also tends to be located within quite discrete domains of inquiry, each with its own 
largely separate literature and audience. ${ }^{3}$ Sometimes these groupings have a disciplinary basis (e.g. applied linguistics, organisational communication, conversation analysis), while others are based on cross-disciplinary communities of interest (e.g. language and gender studies, intercultural communication, health communication).

As noted in chapter 1, there is also a substantial literature in its own right on institutional discourse in relatively formal 'frontstage' settings such as meetings, job interviews, service encounters, courtrooms, classrooms and medical consultations. Embedded within this wider literature on institutional discourse are significant bodies of work focusing on specialised professional settings or technical aspects of workplace (mis)communication (e.g. second language acquisition, conflict and dispute resolution, communication in technical environments, vocational training, the role of communication in systems error and safety and so forth). Comprehensive coverage of all of these areas is clearly outside the scope of this present review, so the main emphasis here will be on providing a brief overview of existing research that most closely matches the focus of this project on theorising and analysing problems with naturally occurring communication in everyday 'backstage' settings such as offices and factories, and the strategies used to manage these issues, along with some discussion of research conducted in 'frontstage' institutional settings of particular relevance to the following chapters.

Almost every example of authentic discourse has several interrelated layers of meaning; when added to the definitional and methodological issues discussed above, this makes a theoretically well-founded categorisation of 'communication issues' somewhat problematic. However, at a commonsense level the literature on problematic workplace communication can usefully be classified into three broad groupings according to the discursive or 'systemic' level of analysis at which the researchers primarily locate the problems in which they are interested (see also chapter 2).

\footnotetext{
${ }^{3}$ Health communication research provides a classic example of the kinds of silos that often develop. In this case there are two very large areas of academic endeavour, language and communication studies in the healthcare setting, and research on health communication based in the social sciences, clinical and health services literatures, which have only really started to connect with one another relatively recently (Sarangi 2004).
} 
The first grouping to be discussed here includes linguistically or interactionally oriented studies of miscommunication or problematic talk which focus primarily on the micro-level analysis of spoken discourse. This is research which explores interpersonal communication processes and practices, and/or mismatches between the communicative norms and discursive practices of different groups. In such studies, communication is often viewed instrumentally as a tool or process, or as the primary medium for getting work done or managing interpersonal relationships in an organisational context (Drew and Heritage 1992b), and the link between spoken discourse and miscommunication is usually made in relatively concrete and transparent terms.

Studies included in the second group focus on language and discourse as a more abstract means of "doing" or contesting power and status in an organisational setting, and/or on cases where problematic communication is linked to more deep-seated underlying political or ideological issues. Here, discourse is seen as being constitutive of institutions and of social identities and relationships. Language and discourse are thus viewed as the primary mechanisms by which interpersonal and intergroup power relations are constructed, changed and maintained, although this is usually an unconscious and less immediately transparent or concrete process.

The final group of studies discussed in this chapter represents more recent research which takes a genre theory or complex systems approach research to miscommunication and communication-related error in organisational and technical settings. Unlike studies in the first two groups in which language or discourse itself is the main target of analysis, here the primary focus is more often on an organisational problem of some kind, with language or discourse as one of a number of systemic human factors involved in the causation or management of such issues.

\subsubsection{Interpersonal and intergroup miscommunication}

As already discussed, there is a great deal of theoretical and applied research in fields such as pragmatics, politeness theory, conversation analysis (CA) and interactional sociolinguistics which focuses particularly on problems of understanding, conversational goal attainment and/or processes of repair at the micro-level of 
interpersonal communication in various kinds of workplace settings. ${ }^{4}$ The kinds of issues examined in this body of interactional research range from relatively straightforward instances of miscommunication or misunderstanding at the most basic level (e.g. cases where the content or 'message' has not been adequately conveyed from one person to another), through to the management of face needs and competing goals in inherently problematic interactional activities (such as disagreement, criticism, advice-giving, complaints, directives, refusals and conflict talk and so on), or the sequential co-construction and repair of interactional dilemmas and 'trouble' (as in conversation analysis).

\section{'Front stage' institutional talk}

Of particular relevance here is a body of micro-analytic research into talk in institutional or organisational contexts which over the past two to three decades has developed into a field of study in its own right. This work tends to be predominantly descriptive rather than critical in the first instance, and although it often has clear "practical relevance" to workplace practitioners (Roberts and Sarangi 1999; Roberts 2001), the researchers' primary interest is usually in developing a theoretical account of the phenomena observed in a range of mainly formal 'frontstage' settings (as opposed to 'backstage' or everyday talk - see above and chapters 1 and 2). For instance, the groundbreaking edited volume Talk at Work: Interaction in Institutional Settings (Drew and Heritage 1992a) includes chapters on problematic talk in job interviews (Gumperz 1992), dilemmas of advice in health visitor interactions with new mothers (Heritage and Sefi 1992), contested evidence in courtroom crossexamination (Drew 1992) and the rejection of advice in a service encounter (Jefferson 1992). Other important collections include Sarangi and Roberts (1999b), which examines discourse in medical, mediation and management settings, Firth (1995) which includes material on workplace negotiation and problem solving, House, Kasper and Ross (2003) which takes an applied linguistics approach to issues of misunderstanding in "high stakes" events such as job interviews, and Heritage and Maynard (2006) which gathers together a range of conversation analytic work on problematic aspects of medical interactions.

\footnotetext{
${ }^{4}$ Previous research in these fields which is of particular relevance to the present study and the wider LWP research programme (see chapter 4.3) has been thoroughly canvassed in previous publications relating to the work reported in this thesis (e.g. Stubbe 1998b, 1998c; Holmes, Stubbe and Vine 1999; Stubbe et al 2001; Stubbe et al 2003; Holmes and Stubbe 2003a), so is reviewed only briefly here.
} 
This body of work also includes research in more general management and organisational settings which investigates a particular aspect of problematic talk. Examples of relevant studies include recent studies of politeness and misunderstanding in service encounters from a conversation analysis perspective (e.g. Olsina 2002; Harris 2003), 'crosstalk' arising from the interaction of language, gender and/or ethnicity in workplace meetings (e.g. Stubbe 1998b; Holmes and Stubbe 2003b; Mullany 2006; Kell et al 2007) and non-cooperation in university supervisory settings (e.g. Ladegaard 2009). A common thread in this work is the need to deconstruct existing models of language and communication to better account for phenomena such as resistance, disagreement or inter-cultural discomfort. Other studies have focused on more explicitly conflictual talk. For instance Jacobs (2002) uses pragmatic implicature theory to describe three tactics used by dispute mediators to manage disputes while maintaining an appearance of neutrality; namely indirect advocacy, framing of advocacy, and equivocal advocacy, which are functional substitutes for the more potentially face threatening moves of direction, correction, disagreement, and argument. Strategies for coping with the difficulties inherent in open-ended problem solving discussions (e.g. Wagner 1995; SollittMorris 1996; Willing 1992, 1997), or with balancing the competing demands of clarity and politeness (e.g. Willing 1992, 1997; Mackiewicz 2002; Howard 2003) are another common focus of more applied research in organisational settings, particularly in interactions involving non-native speakers who are frequently reported to have difficulty with achieving their transactional and social goals in such contexts. Specialised occupational/professional settings

A related strand of research focuses on problematic aspects of interpersonal communication involving occupational or professional discourse in more specialised institutional settings such as health care or aviation. Such studies often have a strongly applied focus on solving real-world problems which goes beyond 'practical relevance' (cf. Roberts 1997), particularly in relation to professional quality and safety issues (see also section 3.3.3 below). Much of this work is based in interactional sociolinguistics or applied CA, although, as noted in chapter 2, this type of research setting also features in recent work in the fields of discursive psychology (see 2.3.4) and rhetorical genre studies (see 2.3.6 and 3.3.3). 
Health interaction research is a case in point. Of particular relevance here are studies of "interactional dilemmas" (e.g. Gill and Maynard 2006; Dowell et al 2007; Dew, Dowell et al 2008; Dew, Stubbe et al 2010) and interactional 'mismatches' of various kinds (e.g. West 1984; Britten et al 2000; Pilnick 2002; Britten 2004; Cicourel 2004; Stevenson et al 2004; Schryer et al 2007; Morgan 2008) in interactions between health professionals and patients which are potentially highly consequential in terms of health outcomes, the quality and safety of clinical decision making and patient satisfaction. A further example of this type of research in recent years is a series of studies by Celia Roberts and colleagues in London which take a multi-pronged "theme-oriented discourse analysis" approach to miscommunication between doctors and patients from non-native English speaking backgrounds, with the aim of developing training strategies based on observations of good practice and misunderstandings (e.g. Roberts, Sarangi and Moss 2004; Moss and Roberts 2005; Roberts and Sarangi 2005; Roberts et al 2005; Roberts 2009).

Close analysis of interactional sequences in the various studies cited above has shown how such mismatches arise in quite specific ways from the interactional complexity and multiple competing demands typical of such settings. Some researchers have now gone on to experiment with and test quite specific interactional strategies and sequences that can be deployed to address such issues in clinical practice. For instance, Stivers (2005) identifies a type of interactional sequence which usually overcomes patient resistance to doctors' treatment recommendations, thus reducing the incidence of inappropriate antibiotics prescribing, and Heritage et al (2007) demonstrate the difference made by the choice of one word rather than another ('something else' versus 'anything else') to the subsequent meeting (or not) of patients' multiple concerns in medical consultations.

Other applied research has investigated the fine detail of miscommunication in "sociotechnical settings where groups or teams coordinate their talk and non-talk activities to perform tasks and complete goals" (Nevile 2004:ix). Relevant studies include micro-analyses of potentially problematic aspects of flight deck exchanges involving pilots and air traffic controllers (e.g. Cushing 1994; Nevile 2004; Howard 2003, 2008), and video ethnomethodological research which explores the ways in which workers accomplish complex practical activities in and through collaborative 
interaction with tools and technology (e.g. Heath and Luff 2000; Heath and Hindmarsh 2002).

Intergroup communication in 'backstage' settings

The research discussed so far involves studies which focus mainly on relatively formal 'frontstage' interactions in professional or specialised technical settings. In such contexts, talk has a central function as the primary means by which the participants' work is carried out. In factories and other 'blue-collar' or vocational settings talk is usually subsidiary to the practical work activities being undertaken and therefore tends to be more sporadic and intermittent; studies of problematic communication in these settings are far less common. Four exceptions to this general observation include a comparative study of directives in two New Zealand factories (Brown 2000), an anthropological linguistics study of interactions between US and Japanese workers in a Texan car factory (Sunaoshi 1999, 2005), an ethnographic study of workplace literacies in a hotel housekeeping team in Canada (Belfiore et al 2004), and a large study of spoken discourse in multicultural workplaces in Melbourne, Australia (Clyne 1994; Neill 1996). This kind of research tends to have a strongly ethnographic flavour as befits its focus on 'backstage' or everyday informal talk in specific work teams or organisations.

The first of the studies mentioned above (Brown 2000) did not focus specifically on miscommunication as such, but nevertheless revealed the problematic nature of even quite routine interactional business such as giving instructions in the high pressure hierarchical environment of a large factory. Sunaoshi's study $(1999,2005)$ used video ethnography and intensive participant observation to describe and unpack the sources of interpersonal and intercultural conflict and misunderstanding between two groups in a Texan car plant, the local Anglo-American shop floor workers and a group of expatriate Japanese technicians, and to identify successful and unsuccessful communication strategies. Belfiore et al (2004) examined how intercultural issues relating to marginalisation and equity were constructed via localised intertextual discourses and everyday interactions. The two related studies by Clyne (1994) and Neill (1996) focused mainly on the pragmatics of intercultural communication in eight different settings, including four factories and a hotel kitchen, yielding 182 hours of recorded data. Somewhat surprisingly, Clyne (1994) identified a total of only 26 instances of "communication breakdown" in this large data set, evenly split 
between problems with understanding the content, the grammar and intercultural pragmatics. Clyne concludes that this is an encouraging finding which suggests Australian workers have become fairly adept at negotiating the pragmatics of communication in intercultural contexts. However, it is unclear precisely where the 'cut-off' lay for identifying something as an instance of communicative breakdown, so it seems likely that this conclusion may in fact reflect a definitional decision rather than the actual occurrence or not of miscommunicative talk in this data set (see 3.2.1 above).

\section{Intergroup communication problems}

Closely related to the above is another important and longstanding strand of applied research in institutional settings deconstructs the communication problems that can occur between people from different social and/or ethnic groups where unequal power relations exist. Such studies often have an explicit aim of identifying practical strategies to combat intergroup conflict and/or discrimination. Researchers sometimes incorporate an action research component, and typically combine microanalysis of interactions with ethnographic methods within an explicitly critical framework.

One well-known and influential example of this type of study is the Industrial Language Training (ILT) project in the UK, a 15-year programme which provided language training to ethnic minority group workers from non-English speaking backgrounds, as reported in Roberts, Jupp and Davies (1992). The theoretical framework for this programme grew out of the work of interactional sociolinguists like John Gumperz, and took account of the links between language and the social contexts of culture and power, and the assumptions and expectations which individuals project into language use. Roberts et al (1992) turned these insights into practical training activities based on the recording and then joint interpretation of workplace encounters. Other more recent work in this vein includes research into the discourse of high stakes gate keeping encounters (e.g. Roberts 2000; Sarangi and Roberts 2002; Holmes 2007; Kasper and Ross 2007; Kerekes 2007a, 2007b).

In another example, Wodak (1996) describes using "discourse sociolinguistics" to solve problems of intergroup communication in a hospital outpatients ward by combining micro-level discourse analysis with longitudinal ethnographic observations. The research made it possible to 'diagnose' typical "inefficient" 
behaviour patterns and negative intergroup attitudes which could be changed relatively easily once they were brought to the attention of the participants as part of an existing training programme for young doctors. A similar 'insider' methodology was applied to the study of a series of primary school board meetings in Vienna, which resulted in a number of specific recommendations as to how these meetings could be made more genuinely democratic (Wodak 1996).

A more recent study set in the intensive care unit of an Australian hospital (Carroll et al 2008) used "video-reflexive ethnography" to iteratively engage nursing staff and physicians in identifying and problem-solving their own communication difficulties. The researchers undertook in-depth, round-the-clock observations, interviewing, and video filming of ward round practices and showed selected video footage to groups of clinicians in structured feedback sessions. This intensive reflexive process resulted in a number of participant-identified improvements to communication processes and practices such as changes to the morning ward round and planning meetings, an increased presence of intensivists on the ward and a handover sheet to improve the structure of clinical information exchanges.

\subsubsection{Competing and conflicting discourses}

This section provides some examples of critically oriented research which illustrates certain discursive strategies or patterns which have been observed to occur at a 'macro-level' as people in workplaces negotiate or contest institutional power and status relationships (see also 2.3.5 and 3.2.2 above). Certain researchers working within this paradigm have taken a particular interest in how the wider 'institutional order' and the interpersonal and/or intergroup interactions which take place at a more localised level mutually influence one another. The approach taken is to integrate macro-level analysis of organisational communication practices and processes with close analysis of interactional and/or textual data (e.g. Fairclough 1989, 1995; Wodak 1996; Thornborrow 2002) in an attempt to "explain why communicative behaviour varies according to the specific structural conditions in which it takes place" (Wodak 1996:7) and to identify as precisely as possible what the various sources of miscommunication or "disorders of discourse" (Wodak 1996) are in particular contexts. 
Wodak and Fairclough are two well-known researchers who have used critical textual analysis in this way in a range of institutional contexts to examine such things as how the creation and propagation of institutional "myths" and the use of metaphor contributes to the miscommunicative phenomenon of "masking" (Wodak 1996). For example, Wodak (1996:39), describing the Vienna outpatients clinic mentioned earlier, concludes that inefficiency, bad organisation and bad training were disguised by the propagation of myths such as "doctors never have enough time", "doctors are never wrong", or "there is simply no better way of doing things". Wodak comments that such stereotypes become all the more powerful when there is some truth underlying them, however minimal.

Metaphors provide us with "models for thinking about social and physical objects, and for communicating a complex set of attributes in a shorthand that can be readily understood" ( $\mathrm{Ng}$ and Bradac 1993:138), and may include the use of formulaic expressions or 'loaded' words, They are created when features from one domain are applied to another, and as Lakoff and Johnson (1980) argue, they do not function simply as stylistic adornment, but rather, are essential constituents of language and thought, and as such, can act as a symbolic site of contention. Norman Fairclough's research into the "marketization" of institutional discourses (Fairclough 1993) has demonstrated how metaphorical practices may frame the discussion of events within organisations in order to encourage a particular interpretation. as well enabling a more deep-seated ideological manipulation by means of the systematic rewording or metaphorising of a domain or the use of "discourse technologies" (Fairclough 1992b).

These processes are seldom made explicit, and, of particular relevance to the present study, the operation of power itself is also often masked by the way a particular discourse or specific interaction is constructed. (See also the discussion of different types of power in 2.3.5). Whilst such "democratisation" of discourse processes in an institutional context (Fairclough 1992b) may produce more understanding and fewer apparent "disorders of discourse" (Wodak 1996), it can also result in the mystifying or masking of power structures in a more skilful way (Wodak 1996:3). An example of this kind of masking of power asymmetries is seen in a study of an organisational change process in a branch of the Australian police (Gordon and Grant 2000). This study found that although consultation meetings were structured in such a way as to 
encourage participants at all levels of the hierarchy to openly voice their views, and superficially gave the appearance of doing so, deeper analysis of the language they used showed marked sensitivity to the existing structures of power and control within their organisation. This continued to restrict the ways in which the people attending actually interacted and contributed their views.

One group can also gain power over another by excluding them from certain aspects of organisational discourse. One exclusion strategy which has been extensively studied is the use of group-specific language. This strategy is equally available to both powerful and less-powerful groups (as also illustrated in chapters 6 and 7 below). It provides a means of creating social distance between one's own group and an out-group, and may, in the process, also create a gap between the minimum information required to make a reasonable judgement on an issue and the information actually provided (Wodak 1996). In a study by Taylor (1987) of work groups in financial institutions, for instance, terminology which may originally have been no more than a handy set of abbreviations also fulfilled several additional functions such as promoting cohesion within its user-group, helping to maintain "desirable" attitudes among existing members, and instilling such attitudes in new entrants to the group (Taylor 1987).

Another example of discursive exclusion strategies is reported by Hallwright (1992) in a case study of interactions between managers and psychiatrists in a New Zealand regional mental health service. She found that managers were often confronted in meetings with highly technical psychiatric jargon, which clinicians seldom made any effort to explain, and which therefore functioned as a type of code used by those with clinical knowledge to communicate about issues such as service priorities. This effectively excluded managers with non-clinical backgrounds from the discussion. For their part, managers used language that was similarly meaningless to clinicians, using phrases like “...we must offer consumer-centred, accessible, equitable, acceptable cost-effective services..." (ibid:121), which were dismissed as irrelevant "management-speak" by clinicians. Managers, too, often introduced technical terminology (e.g. "nett present value calculations") as a way of justifying decisions "...on the assumption that psychiatrists (would) find it hard to challenge their rationale when they do not understand it" (ibid:121). 


\subsubsection{Miscommunication in complex systems}

The application of dynamic systems and complexity theories in the applied social sciences has been gaining momentum in recent years, and the use of such models as a basis for addressing 'real world' communication and organisational problems is similarly becoming more common. This section reviews two examples of research within an organisational communication paradigm where a 'whole systems' approach is applied to the study of miscommunication in workplace settings. As mentioned previously (see 2.3.6 and 3.2.2 above), this analytic approach is a relatively recent development and was therefore not considered as part of the integrative model put forward by Coupland, Wiemann and Giles in 1991, unlike the transmission and individual competency-based paradigms which were predominant in organisational communication prior to the 'linguistic/discursive turn' in the late 1990s.

Examples of two strands of research that fall into this category are canvassed briefly here to indicate the kinds of insights that can be generated by taking a complex systems perspective on miscommunication and problematic talk in organisational settings. The first example provides an illustration of how the tools of rhetorical genre studies and critical inquiry can be used to unpick the rich fabric of situated interaction in an ethnographic study of 'backstage' professional talk. This is work where the primary analytic focus remains on language and discourse. We will then briefly consider a perspective from outside the domain of discourse-oriented studies, namely recent models of error management in organisations where (mis)communication is viewed as simply one aspect of working in a dynamic complex adaptive system.

\section{Genre and activity systems}

As discussed in 2.3.6, studies of problematic discourse which are based on rhetorical genre and activity theories of organisational communication focus on how different forms of communicative practice interconnect to create frames or systems of shared meaning within a workplace (Tietze et al 2003). In the past such research has concentrated largely on the rhetorical structure and stylistic or linguistic features of written texts. However, as already mentioned in chapter 2, there has been increasing interest in studying the ways in which different written, spoken and electronic genres function intertextually and interact to construct the multimodal and overlapping genre and activity systems found in specific institutional and/or professional settings. 
Of particular relevance to the issues of miscommunication and problematic talk being addressed here are: (i) problems of translation between genres (e.g. Schryer et al 2003; DiMatteo et al 2003; Stubbe and Dew 2009; Stubbe et al fc); (ii) the communicative or interactional dilemmas which may arise from contradictions between different activity systems or roles (e.g. Engestrom 1993; Schryer et al 2003, 2007; Dew, Stubbe et al 2010); and (iii) the "ideological consequences" of genre systems (Schryer et al 2003:91). (The latter has clear area of overlap with the critical discourse research reviewed in 3.3.2 above).

An ethnographic study of the day to day work of medical students in a hospital ward by Schryer et al (2003) provides a useful illustration of how problematic aspects of communication can arise out of the interrelationships between the different written and spoken genre systems in this setting. The students observed in this study engaged in multiple discursive activities: they interviewed patients according to a strict protocol, made phone calls, talked with nursing and medical ward staff, consulted and added to patients' notes and medical records, and made case presentations to groups of peers and senior colleagues. The researchers identified several key points at which miscommunication typically arose as information and generically-based interactional patterns moved back and forth, with the students, between these different parts of the genre network.

Firstly, in the course of summarising and synthesising the patient's story into a set of medical notes or a case presentation, important elements could change or become "lost in the translation" (89) between the genres. More specifically, Schryer et al (2003) observed that "breaks in communication" sometimes occurred as a result of the transformation of the patient's 'lay' language into medical terminology. Sometimes this was simply because students used terminology inaccurately or imprecisely. Equally problematic were cases where a medical term was used inappropriately to gloss the symptoms patients had actually described in their own words, thus incorrectly implying a medical diagnosis based on the student's clinical observations. Another kind of problem could arise in reverse, where the student doctor neglected to "undo the transformation" and return to a more accessible linguistic register on returning to the patient to explain their diagnosis or treatment (90). 
Several aspects of the case presentation genre itself were also observed to be problematic. The case presentation as undertaken by medical students or interns is an example of a canonical professional speech genre in medicine which functions as an intermediate step towards fully-fledged case conferencing between colleagues (Erickson 1999). It involves the construction of a routinised 'medical story' in the presence of a supervising senior colleague. This functions as a means of processing and transferring information about a patient drawn from a range of sources at varying times, while at the same time serving as a 'performance' where the student's mastery of the relevant clinical skills and knowledge can be demonstrated and evaluated.

These interactions were observed to be inherently problematic for the students due to the hybridity and resulting "genre tension" (cf. Lingard et al 2003) between two contradictory activity systems ("peer to peer" and "didactic"). One way this manifested itself was in the way the experienced physicians used sometimes quite extended and aggressive interruptions as a strategy to elicit the expected 'medical story' when students were learning to present cases. As well as causing a degree of interactional discomfort at the time, Schryer et al (2003) suggest that this type of genre modelling could have a negative and more long term influence on the way medical students learn to interact with patients, given a reported tendency for medical interactions to be "marred by interruptions" as doctors attempt to elicit the medical facts $(90)$.

\section{Human error and complex adaptive systems}

The management of human error in the interests of safety, quality and efficiency is a prime consideration for professionals in high stakes' organisational and technical workplace settings. Examples of such settings include power plants, military settings, emergency management, and healthcare settings such as operating theatres, emergency departments and intensive care units. Communication is arguably the 'human factor' that is the most open to direct observation and intervention in such contexts, and a number of studies have investigated the nature and extent of communication problems as they relate to actual or potential 'adverse events' in these and similar contexts. The focus is typically on determining the underlying causal factors of a critical incident and/or on designing systems and processes to maximise safety and prevent error. 
Organisational human factors models originally developed out of psychological and engineering approaches to aviation safety in the mid-twentieth century and are now widely applied to safety and quality frameworks in other 'high stakes' working environments characterised by complexity of human interactions and technology, information ambiguity and time pressure. In traditional human error models, as in traditional approaches to organisational communication more generally, the "person approach" or individual deficit model has long been the dominant approach (Reason 2000; see also 2.3.6 and 3.2.2 above). The emphasis in studies taking this approach thus tends to be on reducing unwanted variability and complexity in an attempt to eliminate "communication failures" (Lingard et al 2004) or the "unsafe acts - errors and procedural violations - of people at the sharp end" (Reason 2000:768).

Commonly cited communication issues in this body of work include 'transmission' errors such as missing or mis-timed information (e.g. Lingard et al 2004; Dunsford 2009), information 'overload' or violations of standard protocols (e.g. Howard 2008), and/or interactional difficulties arising from differences in rank or authority and role or expertise, such as the misreading of instructions, failure to resolve disagreements, or reluctance to directly challenge the actions of other team members (e.g. Cosby and Croskery 2004; Howard 2004; Maxfield et al 2005; Evans 2007). These kinds of issues are seen to have a direct and readily identifiable effect on system processes, leading to a range of practical problems such as inefficiency, interpersonal or intergroup tension, resource wastage and procedural error. Typical interventions might include such things as simplifying and standardising procedures, for example by applying structured protocols (e.g. Marshall et al 2008; Salas et al 2008); or checklists (e.g. Lingard et al 2005; Stahl et al 2009); and/or improving communication and coordination within multidisciplinary teams (e.g. Weeks 2005; Maxfield et al 2005; Evans 2007).

This paradigm is increasingly being challenged by advocates of a 'complex systems' approach, in which problems and errors are seen as inevitable and to be expected (though not individually predictable), as consequences rather than causes, and with their origins in "upstream" systemic factors (Reason 2000:769). A prime example of this paradigm shift can be seen in recent health services research into patient/systems safety and clinical error. The crucial importance of effective communication in health care settings and the potential applicability of human factors models are both 
well-recognised in this setting, and critiques of traditional "person-system" error models and interventions based on protocols and checklists (see above) are offered on two main grounds. Firstly, whilst sharing many characteristics with highly technical settings such as aviation and nuclear power, health systems are at the same time far more complex, dynamic and multi-dimensional. As Woods et al (2007) point out, it is the underlying complexity of a system that contributes most to human performance problems, and proposals to improve systems in fact often add complexity to already complex systems, thus potentially making things worse rather than better: "Ultimately, success and progress occur through monitoring, managing, taming and coping with the changing forms of complexity, and not by mandating simple 'one size fits all' policies" (465). Secondly, it is argued that the "person approach" misattributes the majority of systems and communication errors to individuals, a stance that also aligns with critiques of deterministic and/or deficit models of workplace miscommunication. This kind of approach is said to undermine the resilience of the system by thwarting the development of a reflexive "reporting culture" in which people expect to make errors and are trained to recognise and recover them to make the system as robust as possible in the face of its human and operational hazards, which include the vagaries of communication (Reason 2000; Arford 2005). Studies of 'high reliability' organisations from a complex systems perspective have demonstrated that the successful management of potentially hazardous operations involves anticipating and planning for unexpected events (Rochlin 1999, cited in Woods et al 2007:19). Within this paradigm, effective communication is thus seen as integral to maintaining "a consistent mindset of intelligent wariness" (Reason 2000:770). An obvious corollary of this is that actual and potential 'instances' of miscommunication cannot be dealt with in reductionist terms, but must rather be accounted for as dynamic processes within a complex adaptive system.

\subsection{Summary and discussion}

As indicated in chapter 1, the overarching aim of the present study is to explore the proposition that a flexible conceptual framework, incorporating multiple perspectives, analytic methods and data sources, is required to comprehensively address the complexities of 'real world' cases of miscommunication and problematic 
talk in the workplace. This chapter and the previous one have provided a starting point for this exploration with an overview of the theoretical frameworks and methodological approaches most commonly applied to the study of problematic spoken discourse in workplace settings, together with a review of the main existing theorisations of miscommunication, and selected examples of the kinds of research findings generated using these models. This final section summarises the strengths and limitations of existing approaches and research in relation to the aims of the present study, and briefly reviews the case made by some researchers in recent years for taking a trans-disciplinary and/or multi-paradigm approach to researching workplace miscommunication.

This review of the literature has highlighted the wide array of perspectives and methodological tools potentially available to researchers and practitioners to inform their analysis and management of workplace communication issues, and has demonstrated the richness of the practical and theoretical insights already generated within this large body of multi-disciplinary research. Inevitably, however, there are also gaps in the research, as well as a number of problematic theoretical and methodological issues which remain unresolved within the existing literature. These have already been discussed in some detail above, but they will be briefly recapped and drawn together in summary here.

Firstly, despite the wide-ranging nature of existing research on problematic workplace talk, some aspects and settings have received scant attention, either overall or within particular research domains. Of particular relevance to the present study, there is relatively little interactional research with a specific focus on how miscommunication unfolds in everyday workplace settings. As noted earlier, in the majority of cases, the existing research concentrates on more specialised and clearlybounded institutional settings such as doctor-patient interactions, courtrooms, classrooms, formal negotiations and various kinds of gatekeeping interviews, involving selective micro-analysis of single interactions or collections of similar but otherwise unrelated interactions. Studies based on recordings of naturally occurring (mis)communication in everyday 'backstage' settings such as offices, factories, hospital wards and so on are much rarer, probably because it is so challenging to collect and analyse authentic data of this type, and to interpret it without an in depth understanding of the organisational culture and practices in which it is embedded. 
In addition, whilst many interactional researchers do, to some extent at least, incorporate ethnographic methods and data and/or a critical dimension into their analysis of problematic talk, few studies to date involve any systematic 'tracking' of related interactions and texts, or explicit consideration of how communication issues relate systemically to the wider organisational context. This is an important limitation especially for research into miscommunicative talk, because workplace communication systems are typically characterised by dense and multiplex intertextual links, and workplace conversations themselves can often only be fully understood as connected episodes in an ongoing dialogue.

Limiting the data to single interactions or a series of unconnected interactions, as is the case in much micro-analytic discourse analysis, fails to recognise that in most cases miscommunication is in fact a dynamic process rather than a discrete event which is amenable to static or purely localised analysis. Moreover, dynamic processes are inherently difficult to capture analytically (Coupland 2001:20), and even researchers who are concerned mainly with studying discrete instances of miscommunication at the pragmatic level of localised misunderstandings report that, because readily identifiable instances occur unpredictably and sometimes infrequently, it is difficult to collect such data in any systematic way (Tzanne 2000:16).

Conversely, although research in the organisational or professional research domains often does take a more global or systemic perspective, a common limitation here includes a tendency to focus on communication as a linear flow of information, and to identify features of 'unsuccessful' communication at a rather abstract level. Language-in-use is often largely ignored or given a fairly superficial and mechanistic treatment in this literature, with typically little attention to the ways in which people actually 'miscommunicate' through talk in face-to-face interaction. This often results in a narrow 'skills-deficit' view of problematic talk which does not recognise either the complex reality of how such cases evolve, or the sophisticated interactional resources routinely used by people in workplaces to manage their communicative tasks.

A second set of issues relates to the overall lack of theoretical and methodological coherence in the existing literature, a point which has been well-canvassed throughout this and the preceding chapters. On the one hand, as we have seen, the 
existing literature provides a number of tried and tested tools for examining particular aspects of workplace miscommunication from a variety of different perspectives. These all provide their own unique insights, and collectively have generated a large information resource which can be drawn on by other workplace communication researchers and practitioners, albeit with varying degrees of practical relevance and applicability.

On the other hand, this existing body of research is also characterised by a high degree of diversity in the theoretical and methodological approaches used, and in the objectives and contexts of specific studies and research domains, and these differences can be difficult to reconcile. At the most basic level there are complex issues of definition and interpretation around what even counts as miscommunication or problematic talk in the first place, as elaborated in section 3.2 above. Some research in this area is unashamedly eclectic in nature, in some cases to the point of embracing a "radical heterogeneity" (Coupland et al 2001:xiv) which sometimes leads to a lack of theoretical clarity. However, a great deal of the extant literature is also to be found in disciplinary or methodological silos which exist largely in isolation from one another (see also note 3 ). These factors continue to inhibit the cross-fertilisation of ideas and knowledge at a theoretical level, and also mean the potential practical relevance of the existing research effort has not been fully explored or exploited.

As discussed earlier in this chapter, at one level, this diversity of conceptualisations and approaches simply reflects the complexity and ubiquity of the phenomena of miscommunication and problematic talk themselves. Be that as it may, the applied researcher or workplace practitioner is still left with a difficult dilemma: namely, how and on what basis to select from the plethora of information and tools available in order to gain some kind of meaningful and holistic insight into what is 'going on' in a particular case of problematic communication?

The only possible conclusion to be drawn from a review of the available literature is that no single existing approach can, on its own, provide a fully satisfactory and comprehensive account of miscommunication and problematic talk in the workplace. At the same time, as in the parable of the blind men and the elephant, different perspectives and analytic methods may all have valuable viewpoints and insights to offer, even where they are incommensurable or appear to contradict one another. 
Like Coupland, Wiemann and Giles' in 1991, researchers in this field are therefore increasingly arguing that it is necessary to "invok(e) diverse theories and perspectives to achieve comprehensive explanations ... and (come) up with an eclectic model comprehensive and powerful enough to handle diverse cases of misunderstandings" (House 2000:146). The challenge is to determine whether and how it might be possible to draw some of these different strands together into a coherent framework for analysing workplace miscommunication that is at once theoretically robust and also directly relevant and widely applicable to practice.

No eclectic or portmanteau approach has yet been devised which adequately addresses these requirements, including the preliminary 'integrative model' proposed by Coupland, Wiemann and Giles' in 1991, which is the most comprehensive attempt to date. Some workplace researchers have argued that a pluralistic and situated approach is necessary which will allow us to "capitalize on the complexity of in situ practices rather than reduce it to abstracted models or simplified case studies" (Carroll et al 2007:381). Such a multi-paradigmatic approach would provide a more flexible and expansive explanatory framework to draw on for analysing what we can observe happening in actual workplace interactions, while at the same time allowing for an open-ended, emergent and evolving process of theory construction which tolerates apparent contradictions and uses them productively (cf. Harding 1986).

This suggests that what is in fact needed, for both theoretical and practical purposes, is a flexible theoretical 'toolkit' for analysing and managing workplace (mis)communication in workplace settings which utilises the strengths of multiple perspectives without falling foul of "boundaryless thinking” (Richardson et al 2000). One possible unifying conceptual framework for a pluralistic, multi-paradigm approach of this kind that shows some promise is complexity theory as applied to complex human systems. Complexity theorists take it as read that there will be multiple valid representations of the same complex system. A theoretical framework that is sufficiently flexible to tolerate potentially contradictory approaches is thus seen as far more powerful than a model that attempts to somehow integrate or compress different approaches into a single 'grand theory' which would ultimately be reductionist and insensitive to the nuances of particular situations (Richardson 2008). 
As discussed above, the modelling of organisations as (sets of) complex adaptive systems is just beginning to gain currency within the fields of management and organisational communication, largely in relation to more generic issues of teamwork and error management at this stage. With a few exceptions (e.g. Grant 2003; LarsenFreeman and Cameron 2008), complexity thinking has not as yet been widely applied to the study of language and communication. Its applicability to the study of miscommunication and problematic talk in workplaces is therefore an avenue that remains to be explored further.

\subsection{Research Questions}

As already stated, the overall aim of this thesis project is to explore the proposition that a comprehensive understanding of 'real world' cases of workplace miscommunication and problematic talk requires the flexible application of a robust multi-layered intertextual approach which incorporates multiple theoretical viewpoints, analytic methods and data sources. The literature review in chapters 2 and 3 has confirmed that there is considerable scope for further systematic exploration of the nature of miscommunication and problematic talk in 'backstage' workplace settings in general, and for pursuing this line of inquiry in particular. The following specific research questions will be addressed via the analysis of interactional examples and case studies from New Zealand workplaces to be presented in chapters 5 to 7 . These research questions relate in turn to the key theoretical, methodological and practical (applied) objectives outlined in the introductory chapter.

\section{Table 3.1: Research Questions}

\begin{tabular}{lll}
\hline Research question 1 & $\begin{array}{l}\text { a) What kinds of workplace communication problems can be } \\
\text { observed to occur in the New Zealand case study data? b) } \\
\text { What discursive strategies do these workplace participants } \\
\text { use to prevent, manage and repair miscommunication and } \\
\text { problematic talk in their everyday interactions? }\end{array}$ \\
Research question 2 & $\begin{array}{l}\text { What issues arise when we attempt to identify, classify and } \\
\text { analyse examples of miscommunication and problematic talk } \\
\text { in a corpus of naturalistic workplace talk? }\end{array}$ \\
\hline Research question 3 & $\begin{array}{l}\text { To what extent does a pluralistic multi-layered case study } \\
\text { approach 'add value' to the analysis in terms of } \\
\text { theoretical/methodological rigour and practical applicability? }\end{array}$
\end{tabular}




\title{
4 Methodology Analytic approach and research design
}

\begin{abstract}
A workplace participant reflects on the research process:
It was really interesting when you came and did that feedback session with us ... you know light bulbs go on when you do the explanations ... some of it was not unfamiliar to me but to others I think it really was quite a revelation ... yeah and the step beyond that which is, what tools you actually use ... what you could do to change the situation ... if you can identify that something's going on what strategies could you use to get a good outcome? ... ... but it's made me think well are there ways ... are there things you could learn from this research that could help you to work out what is really effective for your own organisation?
\end{abstract}

\subsection{Introduction}

This chapter explains the methodological framework for the case studies of miscommunication and problematic talk presented in chapters 5 to 7 , and describes the research design and methods used to collect and analyse the data. As stated in chapter 1, the specific focus of inquiry for this research developed out of and alongside various studies undertaken by myself and other team members as part of the Language in the Workplace Project (LWP) at Victoria University of Wellington. This thesis focuses on selected subsets of the corpus of interactional and ethnographic data originally collected and analysed as part of that research programme from 1996 to 2003 . The present study is thus positioned as a discrete project embedded within and further extending the methodological framework and research trajectory of the wider Language in the Workplace Project.

The next section (4.2) sets out the basic methodological framework and analytic strategies and tools which underpin the collection, analysis and interpretation of the data presented in subsequent chapters. Section 4.3 provides a summary overview of the original LWP data collection methodology used to produce the data corpus drawn on for the baseline analysis reported in chapter 5. Section 4.4 documents the 
subsequent development, led by the author, of a substantially modified version of the LWP methodology for an applied study of communication within a factory production team. The data collection methodology for this later study was specifically designed to yield the rich intertextual case study data that is examined in chapters 6 and 7 . This is followed in section 4.5 by a summary of the data subsets selected for analysis, placed in the context of the wider LWP corpus from which they are drawn, together with an explanation of the sampling strategies adopted for the purposes of the present study.

\subsection{Analytic approach}

\subsubsection{Methodological framework}

The research reported in this thesis is positioned within a broadly social constructionist paradigm which frames communication as an interactive, collaborative process of meaning making. The analysis is therefore grounded in two basic assumptions. First, interaction is seen as a dynamic process where meanings, intentions and actions are jointly and progressively negotiated between the individuals involved in a given interaction, and social identities, social categories and group boundaries are constantly changing and developing (Weedon 1987; Unger 1989; Butler 1990). In the words of Frederick Erickson:

...what is apparent when we take a close analytic look at the practice of actual interaction among real persons is that both the persons and the situations in which they interact are never fully determined. They are continually in production, under construction, through the boot-strapping processes of contextualisation, shifts in footing, and adaptation by interlocutors to each other's actions. (Erickson 2001:160)

Secondly, it is assumed that a reasonable understanding of the context of an interactional sequence is crucial for interpreting spoken discourse. At one level this includes the kind of information about the immediate situation that can relatively easily be observed or elicited ethnographically: details about the physical setting and purpose of the interaction, the characteristics of the participants and their role relationships, the different channels of communication involved, and the norms or genre(s) associated with it (cf. Dell Hymes' (1974) sociolinguistic "SPEAKING model" of the "elements of communication"). Thus, the interaction in a meeting between officials in a government department will be observably different to a 
troubleshooting discussion between team members on the factory floor. However, there will also be many subtleties that are not accessible to interpretation by an outsider.

At another level, then, understanding something of the background knowledge and perceptions participants themselves bring to an encounter, and the shared experiences and succinct shorthand used by those who habitually work together, can also be crucial in helping the analyst to unpack the layers of meaning and interpret the significance of workplace talk. Finally, it is also important to consider the social meanings of discourse in an even wider context. Workplace interactions in particular tend to be strongly intertextual in nature, and are embedded in the business and social context of particular work groups, as well as in a wider institutional and sociocultural order.

In order to take adequate account of both the wider context of interactions and their dynamic nature, the analytic methodology used here combines the insights and approaches of a number of different theoretical perspectives within a unifying framework of interactional sociolinguistics. This approach was selected as the foundational one because of its critical focus on the interactional and intertextual accomplishment of communicative activities in the workplace context, as one "real world site where societal and interactive forces merge" (Gumperz 1999:454). As discussed in chapter 2 , interactional sociolinguistics is an unashamedly eclectic approach that explicitly straddles the micro-macro divide in discourse studies (see 2.4) by contextualising fine-grained interactional analysis within a broader ethnographic and cultural context. This, together with the proven track record of this approach in researching problematic talk in "strategic" workplace contexts (Roberts et al 1992; see 2.3.3, 3.3.1), means it provides an obvious and appropriate methodological basis for the present study.

As we have seen in the previous chapter, it is possible to analyse miscommunication and problematic talk at many levels and from a number of different perspectives, each of which highlights different aspects of these phenomena. In addition, the LWP data set is extremely diverse in nature (see 4.5 below), and the specific questions which this study seeks to answer are similarly wide-ranging. A multi-paradigm approach is therefore not only valuable, but almost unavoidable for the purposes of this study. Although this inevitably increases the complexity of the analysis, I would 
argue that comparing or combining different approaches in this instance will yield a much richer and more comprehensive analysis (cf. Silverman 1999; Ulijn et al 2000; Stubbe et al 2003).

Additional perspectives and tools from several of the other methodological approaches reviewed in chapter 2 are therefore also integrated into the data analysis within the broad interactional sociolinguistics framework indicated above. As well as making it possible to tease out the potential contributions and limitations of existing conceptualisations of miscommunication and problematic talk, this will serve in this thesis as a way of exploring the utility of a multi-perspectival approach.

\subsubsection{Analytic focus and tools}

The focus of the analysis in this study is first and foremost on the practices participants can be observed to engage in as they manage the problems and complexities of workplace discourse and relationships, whether in the course of a single interaction or tracked through a series of related interactions. As Silverman (1999) suggests, if we wish to learn more about why institutional discourse operates in the way it does, including the various constraints it imposes on the way people interact within organisations, we first need to establish how "participants locally produce contexts for their interaction" (407) by paying close attention to sequential aspects of that interaction at an appropriate level of detail.

This approach is operationalised here via a method known as "thick description" (after Geertz 1973) of the communicative practices involved in a single communicative strand. A "thick description" includes both "fine-grained linguistic analysis ... up and out to broader ethnographic description and wider political and ideological accounts" (Sarangi and Roberts 1999a:1). This encompasses both microlevel analysis (fine-grained linguistic and sequential analysis of individual interactions) and broader, more macro-level, ethnographic descriptions of the communicative patterns and systems observed in the workplace context. The analytic target therefore, is selected interactional sequences identified as typical sites of actual or potential miscommunication and problematic talk within the wider data set, following an adapted version of the systematic procedure set out by Gumperz in his paper 'On interactional sociolinguistic method (1999:465-6). 
The data was approached in the first instance using an inductive, bottom-up, iterative methodology. This involved an initial period of ethnographic research and scanning of the available recorded data and associated material, followed by close analysis of selected interactions in conjunction with any relevant ethnographic information about the contexts within which these were embedded. A 'trace' in either (or both) of these data sources (see 5.2.2) might provide an initial clue that some kind of communicative 'problem' was (possibly) present, thus guiding the selection of data samples for subsequent more detailed analysis, and decisions about the most appropriate analytic focus and tool(s) in each case.

The purpose in this case was to identify instances of actual or potential miscommunication, and from there to build up as complete a picture as possible of 'what is/was happening here?' and 'why and how did it come about?'. The explicit comparison of different kinds of data and the application of different analytic approaches in this way (i.e. data and methodological triangulation), along with the routine practice of taking findings back for comment to the participants involved (i.e. respondent validation) also served to validate (or on occasion challenge) the analyst's initial interpretations (cf. Silverman 2001; Jones and Bugge 2006).

The fine-grained detail of 'key moments' in selected interactions (or sets of interactions) is examined using analytic tools and concepts drawn from conversation analysis and pragmatics, including discursive models of politeness/face and power. Aspects of the ethnography of communication (e.g. Hymes 1974; Gumperz 1982a, 1982b), theme-oriented discourse analysis (cf. Roberts and Sarangi 2005), and applied community of practice models (e.g. Sarangi 2002; Smart 2003), are also used to explore notions of context (cf. Hak 1999; Sarangi 2004) and "ecological validity" (Cicourel 1992, 2003).

A critical perspective is added to the analysis by exploring how the "interaction order" (Goffman 1974) intersects with institutionally ordered social relationships (Cook-Gumperz 2001:119-120), including those traditionally analysed by sociolinguists and critical theorists in terms of social categories or identities such as gender, ethnicity and status. Finally, some relevant constructs from organisational genre studies (e.g. Bazerman 1994; Swales 2004) and complexity theory (e.g. Reason 2000; Larsen-Freeman and Cameron 2008) are also drawn upon to inform the analysis of the factory case study data in particular. (See also chapters 2 and 3). 
These paradigms enable a sharper analytic focus on the intertextual nature of different (mis)communicative episodes and the complex and dynamic practices and activity systems within which these are embedded.

An integrated approach such as this makes it possible to relate what is happening sequentially and "on-line" during particular interactions to wider issues such as team or organisational culture, group membership and the wider socio-cultural context. As will be demonstrated in chapters 5 to 7 , a deliberate articulation in this way of (sometimes cross-cutting) insights from micro- and macro-level analyses focusing on different aspects of the same data makes it possible to go both 'wide' (thus identifying generalisable themes and patterns), and 'deep' (thus allowing for in depth analysis of critical incidents). This provides an integrated, richer and more nuanced analysis than would be possible by using a single approach or data type. Depending on the evidence available in the data, such an analysis may also allow a dynamic reconstruction or deconstruction of the problematic communication under examination.

The remainder of this chapter provides an account of the unique participatory data collection methodology developed by the author in collaboration with other members of the LWP team in a variety of different workplaces (section 4.3), and its subsequent adaptation for tracking communication in a factory team (section 4.4). ${ }^{1}$ This is followed in section 4.5 by a description of the particular data sets drawn on for this study, and the two stages of analysis reported on in the subsequent chapters. This account draws on a variety of published and unpublished material produced by the author and others, in which different aspects of the LWP methodology are described as it evolved over time, (See in particular Stubbe 1998a, 2000a, 2001; Stubbe and Ingle 1999; Holmes and Stubbe 2003a; Jones and Stubbe 2004).

\footnotetext{
${ }^{1}$ Maria Stubbe and Janet Holmes developed and piloted the original methodology in four government workplaces during 1996 and 1997. Subsequent adaptations for different workplace contexts were undertaken progressively from 1997-2003, including corporate project meetings (Meredith Marra, Janet Holmes, Bernadette Vine) and small business and factory environments (Maria Stubbe, Rose Fillary, Pascal Brown, Janet Holmes).
} 


\subsection{The original LWP data collection methodology}

The workplace is a highly complex and diverse social setting, where the practical demands of the business at hand inevitably take precedence over other more peripheral concerns. Between 1996 and 1998, I co-led the development with Janet Holmes of the original LWP data collection methodology, an innovative participatory approach which was designed to meet the practical and ethical challenges of collecting a large corpus of naturally-occurring spoken data which would provide a representative snapshot of the ways in which people talked in a variety of workplaces in New Zealand (Stubbe 1998a, 2001). This section describes the key features and design principles of the original LWP methodology, and concludes with a brief evaluation of the strengths and limitations of this data collection model for the purposes of studying miscommunication and problematic talk in the workplace.

\subsubsection{Designing the methodology}

As already mentioned in chapter 1, the Language in the Workplace Project (LWP) began in 1996 with two broad goals in mind: (1) to analyse the features of effective interpersonal communication in a variety of workplaces from a sociolinguistic perspective; and (2) to explore the practical implications of these findings for New Zealand organisations. (See Holmes and Stubbe (2003a) for a detailed overview of the project as it evolved from 1996-2003.) In designing the project methodology, the team kept these basic objectives in mind while at the same time weighing up how best to deal with a range of practical issues and constraints which impacted on the research process.

In the first instance it was necessary to devise a practical method of collecting a reasonably representative database comprising high quality, natural interaction data and associated information from everyday workplace contexts. This data set had to be of sufficient size and quality to provide a suitable basis for detailed discourse and pragmatic analysis, and to allow explicit account to be taken of socio-cultural factors such as gender, culture, and relative status and power relationships. However, the process of data collection could not be overly intrusive, both in order to avoid tape shyness and to minimise disruption to the normal flow of work and interaction. The 
research process also aimed to be as open and empowering for participants as possible to avoid any possible exploitation or misrepresentation of informants.

Secondly, the LWP team were committed to undertaking a programme of applied research in collaboration with participating workplace practitioners with the aim of achieving specific and generalisable "real world outcomes" (cf. Roberts and Sarangi 2003). Right from the start of the project, therefore, an ongoing dialogue was established with the individuals and organisations involved, and the research design was based as far as possible on the action research principle of "research on, for and with" participants (Cameron et al 1992:22) within an explicitly "appreciative inquiry" framework (Hammond, 1996; Jones and Stubbe 2004). Appreciative inquiry is an organisational learning approach which involves looking for what is done well, with the aim of finding ways to share strengths with others and develop them further, as distinct from looking for 'problems' and setting out to 'solve' them. Because it was difficult to predict at the beginning exactly what form this collaboration might take in a given organisation, the methodology had to be sufficiently flexible and adaptable to evolve with the project.

Thirdly, the research design had to meet stringent ethical requirements, the most immediate of which were ensuring that genuine informed consent was obtained from everyone who was recorded, and guaranteeing confidentiality to the individual informants and organisations involved in the project. ${ }^{2}$ These are of course quite usual considerations in any research involving human subjects, but they acquire an extra edge in the workplace context, where people are very aware of the need to protect sensitive information, and to preserve their relationships with clients, colleagues and managers. Even though they knew that the content of their interactions would not be the main focus of the research, participants were initially very wary of losing control over any data that could potentially identify and compromise either individuals or the organisations concerned. It was also important to ensure that people did not feel pressurised into giving their consent to be recorded simply because their colleagues or managers had already agreed to be involved in the project.

\footnotetext{
${ }^{2}$ See Appendix 2 for details of the ethical approval process and samples of the research documentation given to research participants.
} 


\subsubsection{Basic components of the participatory model}

The key feature of the original LWP methodology was its non-intrusive participatory approach in which volunteers from a particular team or section of an organisation undertook to collect the data themselves with technical and logistical support from the research team. The basic data collection model moved through four distinct stages: (i) making contact and establishing the research relationship; (ii) collecting ethnographic information and recording talk; (iii) initial processing and analysis of the data; and (iv) providing reflexive feedback. This four-stage iterative process provided a basis for the research team and participants to collaborate in setting the research agenda and to exchange relevant information, and for the people and organisations concerned to subsequently reflect on and develop their own communication practices. (For a full description, see Stubbe 2001; Jones and Stubbe 2004).

During the data collection phase, interactions were recorded as unobtrusively as possible (but always with the explicit knowledge and agreement of all involved). This meant reducing the researchers' direct involvement in the physical collection of data to the minimum possible in a given workplace. Typically, individuals from within the organisation agreed to carry an audio recorder and/or microphone to selectively record samples of their everyday interactions at work over a period ranging from two or three weeks to several months. This approach yielded a large amount of rich natural data, with participants reporting that the recordings were by and large typical of their routine interactions. Demographic and ethnographic data were collected by a variety of means, including initial discussions with managers, workplace observations, briefing and debriefing sessions with volunteers, contextual notes provided by the participants at the time of recording, and follow-up interviews with selected participants involving the reflexive analysis of data extracts.

Another vital component of the participatory approach was giving ongoing opportunities for face-to-face debriefing on issues raised by the participants during and immediately after the recording, and providing early relevant feedback in the form of seminars, reports and practical workshops based on the initial data analyses carried out by the researchers. These sessions targeted the evaluation and development of selected aspects of workplace communication, focussing particularly on areas which had been identified as relevant by participants, such as meeting 
processes, the communication styles of managers, and the relationship between gender or ethnicity and workplace language. During the intensive data analysis phase of the project, we also undertook follow-up interviews with selected informants involving the reflexive analysis of data extracts and summaries. This follow-up functioned as a way of supplementing the contextual information, checking that the analysts' interpretations were on track, trialling some of the ways the project data and our analysis of it might be applied, and providing further opportunities for feedback in both directions. By engaging in a two-way dialogue throughout the research process, leaving control of the recorded material in the hands of informants and guaranteeing anonymity and confidentiality, the research team developed an excellent research relationship with participants.

This methodology also aligns neatly with the action research cycle (Wadsworth 1988), illustrated in Figure 1 below, with successive iterations allowing for the exploration of new research questions and the development of practical applications, tracking back to one of the previous stages as appropriate. ${ }^{3}$

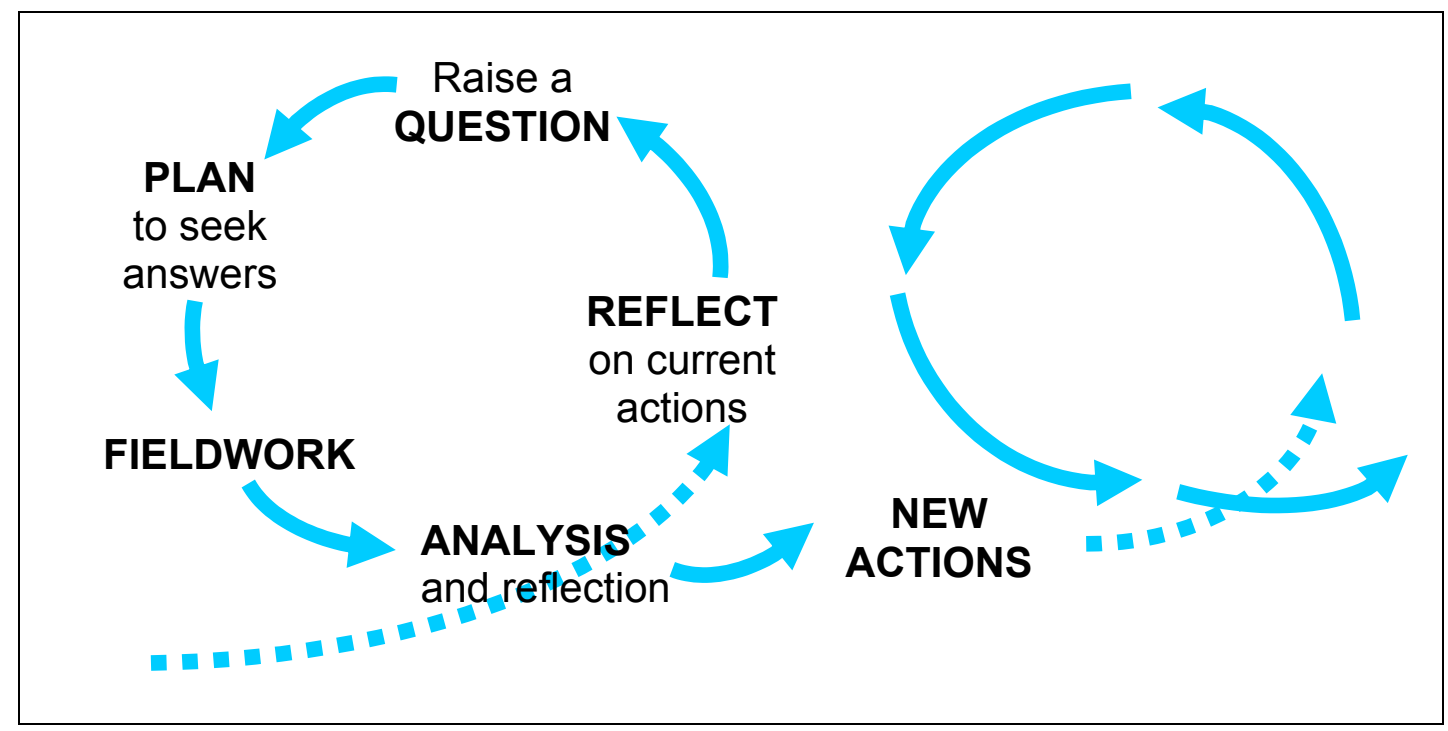

Figure 4.1: The action research cycle $^{4}$

Between 1996 and 1999, the LWP research team progressively developed and finetuned the participatory methodology outlined above in a range of different workplace settings. (See sections 4.3.3 and 4.5 below for details of the data collected).

\footnotetext{
${ }^{3}$ See Jones and Stubbe (2004) for a fuller discussion of this aspect.

${ }^{4}$ Adapted from Wadsworth (1998)
} 


\subsubsection{Original data collection and projects}

The original data collection was undertaken during 1996-1998 in four New Zealand government agencies. In each organisation, a group of workers representing a range of roles and levels within the organisation agreed to identify and record a representative range of their everyday interactions at work, totalling up to four hours over a period of two to three weeks. They were asked to record samples of all the different kinds of interaction they engaged in at work, including social chat and telephone calls, as well as more obvious kinds of work-oriented talk. Some more formal meetings were also recorded on video, with the organisations supplying documents related to the meetings such as agendas, minutes and other relevant background information. Ethnographic data were also collected by means of workplace observations, contextual notes provided by the participants at the time of recording, briefing and debriefing sessions with volunteers, as well as general background information provided by the management of each organisation.

During 1997-98, LWP researchers also worked separately with three large private sector organisations to record a number of regular project team meetings over a period of several months. The basic principles of the methodological design remained the same, although the data collection methods required a degree of adaptation to enable good quality video and audio recordings of these larger, more formal meetings to be collected. ${ }^{5}$

In addition to seminars and workshops in each participating organisation carried out within 6-9 months of the data collection at each site, an action research project was subsequently carried out in 1999 with groups of workers from two of the participating organisations, a government department and a private sector organisation,These groups collaborated with LWP researchers ${ }^{6}$ to trial a communication evaluation and development (CED) model and training toolkit based on reflexive action learning principles. This tool built on the government and corporate workplace communication data collected and analysed from 1996-1998 (see Jones and Stubbe 2004 for a full report on the development of this model. Other relevant information can be found in Stubbe 1997a, 1998b, 1998d, 2002; Jones 1998,

\footnotetext{
${ }^{5}$ This data was not included in the analysis for this project.

${ }^{6}$ The development and trialling of the CED model and tools were led by Maria Stubbe and Deborah Jones, assisted by Bernadette Vine and Meredith Marra.
} 
2001, 2002; Holmes and Stubbe 2003a). The rationale and principles underlying this model as they relate to the current project are discussed further in section 5.2.1 of the next chapter.

From 1997 to 1999, two LWP research associates, Rose Fillary at Eastern Institute of Technology (EIT) in Hawkes Bay and Pascal Brown at UNITEC in Auckland, used a similar methodology to collect audio recordings from shop floor and office workers in a range of small businesses and factories as an evidence base for developing communication training tools for workers in supported employment (at EIT) and vocational English as a second language curses (at UNITEC). These workplaces included a plant nursery, a building recycler, and an early childhood education centre that provided work placements to workers with an intellectual disability (Fillary 1998; Holmes et al 2000), a large hide tanning factory, and a shoe factory (Brown 1998, 2000; Stubbe and Brown 2002).

Unlike the professional office and meeting environments described above where talk is not only integral to the core business of the workplace, but often is the work to a great extent, the work in these business and shopfloor settings was more practically based, and talk was more often transactional - a means to an end. The data collection methodology was modified accordingly to overcome various practical and logistical barriers to the recording of spoken interaction such as noise, safety issues and more unpredictable and sporadic communication patterns; however, it still retained the snapshot sampling approach used previously in the government and corporate office settings. These latter projects also incorporated the development and piloting of vocational and language education tools and resources.

\subsubsection{Strengths and limitations}

The original LWP methodology was used to collect data for a series of related projects between 1996 and 1999 as outlined above (see Holmes and Stubbe 2003a for a full review). This work produced a rich open-ended corpus of authentic workplace data which has continued to be drawn on for both theoretical and applied research. (See for example: Holmes, Stubbe and Vine 1999; Stubbe and Homes 2000; Stubbe et al 2001; Stubbe and Brown 2002; Stubbe 2002; Stubbe et al 2003; Marra 2003; Brown and Lewis 2003; Vine 2004; Daly et al 2004; Holmes and Stubbe 2003b, 2003c; Holmes and Stubbe 2004; Holmes 2006; Vine 2009; Vine et al 2009). 
Over the past ten years the same basic methodological principles have also been adapted and extended to a variety of new settings and topics by members of the original LWP team and affiliated researchers in NZ and internationally. Examples include work in the following areas: healthcare interactions (e.g. Macdonald 2002; Stubbe 2004; Dowell et al 2007; Major and Holmes 2008); vocational language and communication (e.g. Stubbe and Brown 2002; Cooke et al 2007; Fillieatz 2009) organizational knowledge creation (Fletcher 2003); workplace email (Waldvogel 2005); leadership and mentoring (e.g. Holmes and Marra 2004; Chiles 2006, 2007; Chan 2007; Vine et al 2008; Schnurr 2009); technical communication (e.g. Angouri and Harwood 2008); international corporates (e.g. Schnurr 2009; Schnurr and Chan 2009); and telephone complaint lines (Weatherall and Stubbe 2009). This participatory data collection model has thus proved to be extremely productive, as well as robust and adaptable to many different settings.

The original LWP methodological design nevertheless also had certain limitations. In particular, the strategy of handing over the responsibility for recording to participants, and the resulting open ended sampling, inevitably reduced researcher control over what data was actually recorded and the overall composition of the data set, which typically comprised self-selected convenience samples or snapshots of the key informants' everyday communication over a relatively short period of time. As discussed earlier, there were good theoretical and pragmatic justifications for proceeding in this way, including the creation of a wide-ranging and diverse corpus, which can be drawn on for many different purposes.

However, one general consequence is that data sets collected in this way have gaps and inconsistencies, which may prevent or impede analysis of certain communicative phenomena. For instance, in the original LWP projects, participants tended to view anything they defined as 'off-task' talk as being unimportant; as a result, they regularly failed to record instances of social chat or other interactions they considered to be 'uninteresting' or merely routine, and there were also certain categories of interaction that were seldom collected due to practical or ethical constraints. This made it difficult, for example, to do a reliable analysis of certain phenomena such as the distribution of small talk at the beginning and ends of meetings, or to examine certain kinds of routine interaction such as telephone calls or 'corridor chat'. 
A second limitation of the methodology as originally implemented is that it generally produced a number of largely unrelated one-off recordings, with no particular topical framing or links, and often with little ethnographic data or other information to contextualise the interactions for an outside analyst. For many purposes, such constraints are in fact analytically unimportant. Indeed, the varied and wide-ranging nature of the data sets collected as part of the original project arguably made them a more valuable resource for my initial exploratory analyses of miscommunication and problematic talk than a less open-ended data set would have been. As set out in the next chapter, this diversity provided an important springboard for further developing the analytic framework set out in section 4.2 above, and which informs the analysis of the case study data in chapters 5-7.

However, as will be demonstrated in the next chapter, it soon became apparent that it was the exceptions to the general pattern of one-off' recordings that afforded the most important insights with respect to identifying and understanding instances of miscommunication and problematic talk. For example, participants would sometimes record two or more topically linked interactions, which made it possible to a limited degree to follow the action over time and/or with different participants. ${ }^{7}$ Alternatively, they might collect a set of interactions where the same participants were talking to one another across different contexts, thus allowing more systematic intertextual comparisons to be made (e.g. Stubbe 1998b; Stubbe and Holmes 2000; Holmes and Stubbe 2003b). Participants sometimes also volunteered pertinent institutional or contextual information and reflections in post hoc debriefings or feedback meetings, which allowed the research team to (re)interpret recorded interactions with greater accuracy (e.g. Stubbe 1997a, 1998d; Jones and Stubbe 2004).

These were incidental bonuses, fortuitous rather than outcomes deliberately built into the original research methodology. As the initial analysis for the present study proceeded, it became increasingly clear that the somewhat random retrospective data sets produced using the original methodology would be inadequate to meet the more specific objectives of the next stage of analysis, namely to test the utility of the

\footnotetext{
${ }^{7}$ One striking example of this occurred when an informant with an employment-related grievance recorded both a full and frank discussion about it with a friend over lunch, and also a subsequent lengthy meeting with a senior manager about the same issue (see Stubbe et al (2003) for an analysis of this latter interaction).
} 
analytic model developed during the first stage of the analysis (see chapter 5), and to explore in detail how problematic aspects of workplace interaction are managed within a particular workplace setting (see chapters 6 and 7). I concluded that the most efficient and illuminating way to achieve these objectives would be by means of a purpose-designed case study to allow a "thick description" of a set of intertextually linked interactions, as discussed in 4.2 above (cf. Sarangi and Roberts 1999; Stubbe et al 2009). The next section (4.4) explains how the original LWP methodology was adapted to meet the requirements of these more specific research objectives, and also to accommodate the 'talking conditions' and technical constraints encountered in a very different type of workplace setting: a factory production team.

\subsection{Action research with a factory team: the adapted methodology}

The data collection methodology described above was devised principally to meet the practical and ethical challenges of collecting a large corpus of spoken interaction data from a number of government and corporate workplaces during the first stage of the Language in the Workplace Project. This section describes how the original methodology was subsequently adapted by the author to cope with a new set of research objectives and a rather different working environment, thus making it possible to intensively track the communication within a production team in a large factory. ${ }^{8}$

Motivated by information about the first three years of the research as described above, a manager from the Unilever soap products plant in Petone ${ }^{9}$ approached the LWP team with a proposal to collaborate on a piece of action research into the effectiveness of communication in one of the work teams at their factory. The

\footnotetext{
${ }^{8}$ The intensive data collection methodology for this factory project described in 4.4.1.and 4.4.2 below was designed by Maria Stubbe, and implemented with the invaluable support of a research assistant, Megan Ingle. Maria Stubbe had overall responsibility for all aspects of the on-site fieldwork; she recruited the participants, conducted the initial observations and interviews, and visited the research site regularly throughout the data collection period and beyond to collect ethnographic data, liaise and conduct interviews with the participants, and facilitate feedback sessions. Megan Ingle was employed to stay on-site throughout each period of active data collection to manage the technical and logistical aspects of the recording process, to track and debrief the participants being recorded, and to undertake systematic observations. (See Stubbe and Ingle 1999 for a more detailed description).

${ }^{9}$ This company was happy for their association with the LWP research to be made public.
} 
management of actual and potential communication problems in a multicultural selfmanaging shop floor team quickly emerged as an appropriate focus for investigation, given the perceived importance of effective communication in optimising safety, quality, productivity and workplace relations in a very challenging environment. (See chapter 6 for a full description of this team and its working environment). This collaboration provided an excellent opportunity to collect the kinds of intensive interactional and ethnographic data required to meet the specific objectives of the next stage of the miscommunication study (as discussed in 4.3 .4 above). A protocol for a pilot study was agreed upon, and the factory's top-performing production team was recruited to take part in this pilot project and subsequently also in the ongoing doctoral and action research programmes.

\subsubsection{Developing the case study methodology}

The factory project was designed with multiple goals in mind. The team co-ordinator (TCO) was particularly interested in further improving communication within her own team, while the factory's human resources and training managers wanted information that they could use to assist other production teams lift their performance to a similar standard. Members of the LWP team, including myself, aimed to use both the data collected and the action research process itself as a basis for developing a more widely applicable communication evaluation and development model for use in New Zealand workplaces. This would extend work already done in pilot projects in several white-collar workplaces (see above, and also Jones and Stubbe 2004). An additional and more specific objective was to identify and track instances of miscommunication and problematic talk by building greater depth and intertextuality into the data collected than had been the case previously. A longitudinal triangulated case study methodology was developed to achieve this set of related objectives, as described in section 4.2.2 above.

Initial discussions with the TCO and a tour of the very busy and noisy factory floor quickly made it obvious that the technical and logistical aspects of the existing methodology would also require significant modification in order to overcome the challenges inherent in collecting natural interaction data in this environment. For instance, we had to develop new strategies for identifying and collecting a useful and representative sample from a dispersed workforce whose engagement in face-to-face interaction was typically quite limited. We also had to devise appropriate ways of 
physically collecting recordings and essential contextual information about each interaction in an extremely busy and noisy environment where the workers moved around constantly. In addition, we faced some tricky ethical issues around the relationship between the research team, factory management and the participants themselves. These issues included gaining the trust and co-operation of team members, ensuring all recording and note taking was done with informed consent, and satisfying ourselves that this consent was freely given, and not simply out of compliance with management wishes.

A key objective was to retain the participatory and reflexive approach that characterised the existing methodology, and to ensure that individuals still had as much control as possible over whatever data was collected from them. However, the research team clearly had to be much more hands-on in the data collection process than had been the practice previously. It would not be possible to hand over the task of selecting and recording interactions to workplace volunteers as we had done elsewhere, because this sort of activity is simply not compatible with the nature of work in a factory.

Therefore, rather than relying on key informants to record their own self-selected convenience sample over a period of several weeks as previously, a more traditional participant observation strategy was adopted. This involved having an on-site researcher embedded in the team to collect data intensively from selected staff over the course of a complete shift, recording as many spoken interactions as possible in that space of time. Detailed structured observation and the collection of other kinds of related data such as interviews and documents were also undertaken to maximise the likelihood of related topical themes and contextual information emerging to triangulate the data and enrich the analysis.

\subsubsection{Data collection}

Data collection in the form of participant observation, tracking and audio recording ${ }^{10}$ of this team's communication was undertaken over two separate periods. In the first pilot stage, the fieldworker was present at the factory for a rolling three to four hours a

\footnotetext{
${ }^{10}$ Video recording was specifically excluded as an option by the management for reasons of commercial sensitivity, but would in any case have been very intrusive and difficult to manage from a practical perspective with the large VHS cameras which were the most up-todate technology in 1999.
} 
day over successive shifts, in order to obtain samples from each part of a typical day and each day of a 4-day shift period, ${ }^{11}$ and to gather baseline ethnographic data to feed into the collaborative action research project. The second stage of this project, three months later, involved more intensive data collection for six full twelve-hour days. This included recording and observing a complete four-day shift cycle, plus a day at the end of the previous cycle and one at the beginning of the following cycle. The fieldworker remained on-site throughout each recording period to change audiodiscs and batteries, write up interaction notes, obtain ethnographic information, and begin data processing. This meant balancing the practical requirements of data collection (e.g. servicing equipment, recording contextual information) with being as unobtrusive as possible in order to avoid interfering with the team's usual patterns of work and communication. ${ }^{12}$ This multi-component method of data collection produced a very rich, dense and multiplex data set comprising over 800 interactions (approximately 60 hours of recorded speech) from 31 participating factory staff, with excellent potential for data triangulation and intertextual analysis.

Adding to the complexity of the fieldwork was the fact that the production team in question worked in two separate areas of the factory, located physically one above the other and linked by a series of powder delivery chutes. The first was a manufacturing area, where operations were monitored from a computerised control room. Workers constantly moved in and out of this room, and a radio intercom to the factory floor was in regular use. The second area was the packing line where workers moved around only to monitor machinery, and talk tended to be more intermittent, functioning mainly to impart specific information or instructions. Staff from these two areas did not typically interact physically during the course of the day. Different methods of recording and obtaining informed consent were thus required in each of these settings. The team coordinator moved between both of these areas and management offices in a completely separate location, and other workers such as engineers, fitters, quality assurance and stores staff also moved in and out of the area to interact with the team on which we were focussing.

\footnotetext{
${ }^{11}$ The production and packing teams at this factory worked 12 hour shifts on a 'four days on, four days off' roster.

${ }^{12}$ In fact, the fieldworker blended in so well in her role of participant observer that she was eventually offered a job in the factory.
} 
In the control room, the best quality of recording was obtained using a portable digital minidisc recorder attached to a Soundgrabber microphone. For particular individuals in static and less noisy settings such as the morning briefing sessions, the fieldworker set up a minidisc recorder in a waist pouch, together with a high quality omni-directional lapel microphone. In these contexts, agreement to record was obtained on every occasion before the recorders were switched on. For workers who moved about the factory floor, one or two key individuals carried radio microphones for two to three hours at a time on a rotating basis, transmitting to a minidisc recorder in a suitable location monitored by the fieldworker. The latter produced good results, especially in situations with a lot of background noise, and had the added advantage that the person 'wired up' was not constantly reminded that they were being recorded by the need to change discs. In fact, minidiscs provided an extended recording time of 148 minutes, together with a full random access editing capability, which proved invaluable for data processing in a situation where there were often long intervals between interactions. ${ }^{13}$ The changing over of microphones provided a natural point for the fieldworker to debrief participants and gather background information, with people generally reporting that they had quickly forgotten about the fact they were being recorded. As in all previous recording, participants had the right to ask for material to be deleted or to veto the use of any talk they did not want used for analysis, but in practice this very seldom arose.

Because the project undertaken in this factory was framed very explicitly right from the start as action research, feedback to the participants and to other interested parties such as human resources and supported employment personnel was an integral part of the process. The author as the principal researcher from the LWP team, had regular meetings with the team co-ordinator and with training and human resources staff to explore how the results of the research could most usefully contribute to team development programmes at the factory. The team themselves also had a number of opportunities to interact informally with members of the research team and were invited to two formal feedback sessions where they were able to hear samples of the

\footnotetext{
${ }^{13}$ This type of data collection would have been much more difficult without affordable digital audio recording devices - previous LWP projects made use of Sony 'Walkman' cassette recorders which were at that stage the most practical technology.
} 
recordings and discuss how the material might be used. ${ }^{14}$ These discussions were also recorded, and doubled as an additional source of ethnographic data.

\subsection{The data: from office to production line}

By 2003, the full LWP corpus comprised approximately 1500 interactions collected in 14 different workplaces from 420 people of a range of ages, ethnic groups and occupations. These had been recorded in five government and three commercial white-collar organisations, as well as in four small businesses, two factories and a hospital ward (Holmes and Stubbe 2003a:13). This corpus includes social talk recorded in workplaces as well as task-oriented talk, and the length of recorded interactions ranges from short telephone calls and brief interactions of less than a minute to long meetings which last more than four hours. The data samples for the two analytic stages of this doctoral project (the baseline analysis and the factory case study) were drawn from two sets of data from the full LWP corpus as detailed in Table 4.1 below.

TABLE 4.1: LWP Data Sets (Number of recorded interactions)

\begin{tabular}{|c|c|c|c|}
\hline \multicolumn{2}{|c|}{$\begin{array}{c}\text { Stage } 1 \text { Analysis } \\
\text { BASELINE DATA SET }\end{array}$} & \multicolumn{2}{|c|}{$\begin{array}{c}\text { Stage } 2 \text { Analysis } \\
\text { FACTORY DATA SET }\end{array}$} \\
\hline Government 1 & 134 & Packing line & 496 \\
\hline Government 2 & 146 & Manufacturing & 83 \\
\hline Government 3 & 20 & Team coordinator & 226 \\
\hline Government 4 & 33 & Shift briefings & 8 \\
\hline Small business & 90 & Miscellaneous & 23 \\
\hline Totals: & 423 & Totals: & 836 \\
\hline
\end{tabular}

\footnotetext{
${ }^{14}$ Subsequently, members of this team were involved in the filming of a video produced as part of a generic training resource kit (Talk that Works) aimed at the development of effective communication in multicultural factory teams (Stubbe and Brown 2002).
} 


\section{Stage 1: Baseline data set}

The initial exploration of a possible analytic framework for this study was based on a reference sample drawn from a baseline data set of 423 interactions and related data collected as part of the original LWP project from 1996 to 1998. A total of 333 of these recordings came from the policy and advisory units of four government organisations, an environment where talk is integral to the core business of the workplace. These were the first workplaces to be involved in the LWP research, and they were deliberately selected to include one organisation with a high proportion of women and one with a high proportion of Maori workers, in addition to two workplaces with an ethnic and gender balance more closely reflecting the New Zealand norm. ${ }^{15}$ The bulk of this data consists of small, relatively informal workrelated office meetings and discussions involving people from a range of different levels within each organisation. Such interactions fulfilled a wide variety of purposes in these workplaces: to plan, to convey instructions, to seek advice, to check reports, to solve a problem or do a task, to provide feedback, to evaluate proposals, and so on. An additional set of contrasting data, consisting of 90 interactions recorded in several small businesses in Hawkes Bay also formed part of the baseline data set. This baseline data was complemented by a small amount of ethnographic case study data from action research pilots undertaken in one of the government workplaces, and one of the organisations that had participated in the study of corporate meetings. (See section 4.3.3 above).

\section{Stage 2: Factory data set}

The data for the factory case study comes from the two periods of fieldwork described in section 4.4.2 above. In all, 836 useable interactions were recorded at this site, involving 31 participants, 21 of whom were from the production team that was the focus of the study. The total recorded time was approximately 60 hours. The shortest interaction was 8 seconds and the longest was 1 hour, 47 minutes, but the majority clustered around a half minute to two minutes in length. The recordings were collected in three main ways. Over half of these interactions (496) were

\footnotetext{
${ }^{15}$ As at the 2006 Census (Statistics-NZ 2010), the majority of the New Zealand population of 4.3 million (just under 70\%) was of Anglo- or Western European origin (known locally as Pākeha). The indigenous Māori population was the single largest ethnic minority comprising approximately $15 \%$ of the population, followed by Asian (9\%) and Pacific (6.5\%) ethnicities. These percentages are similar to those recorded in the late 1990s when the data collection referred to here took place.
} 
recorded by different team members who wore radio microphones as they worked on the packing line. A much smaller number of interactions (83) was captured on the static recording equipment in the manufacturers control room upstairs, mainly because it was not feasible to record these workers whilst 'on the move' for safety reasons. A further 226 interactions were recorded in various locations by the team coordinator who carried a recorder and lapel microphone right throughout the data collection period, and recorded the majority of the interactions in which she was involved. This latter set of interactions provided the focus for the second part of the case study analysis presented in chapter 7 . The remaining 23 recordings are incidental interactions about workplace issues between a range of different factory staff and the fieldworker in her participant observer role.

\section{Data analysis}

As discussed earlier in section 4.2.2, interactions or sequences of interactions were purposively selected from these data sets for more detailed analysis on the basis of clear interactional and/or ethnographic evidence of miscommunication or problematic talk. Chapter 5 details the first stage of this analysis. Illustrative analyses of a reference sample of critical incidents are used here to evaluate and refine existing conceptual models, and to outline the initial specifications for a multiperspective framework for defining and analysing miscommunication and problematic talk. Stage 2 of the analysis is presented in chapters 6 and 7 where elements of this proposed approach are further elaborated by means of the in-depth examination of case study data from the factory data set. Additional reference will also be made to previously published analyses of related examples as relevant. 


\title{
5 Developing a working model What exactly is miscommunication anyway?
}

\author{
Two colleagues troubleshoot a workflow issue: \\ Jan: I think that's the best thing \\ it hasn't been signed by Philip because he wanted that information included \\ Heke: all right okay [sighs]: oh look: I'm dreadfully sorry about that \\ Jan: oh well just check out to see what happened 'cause there was clearly some \\ miscommunication somewhere
}

\subsection{Introduction}

From a lay perspective, terms such as 'miscommunication' or 'problematic talk' are typically taken at face value and interpreted according to commonly accepted definitions such as "failure to communicate clearly, fully, or accurately" (OEDOnline 2010). However, as we saw in chapter 3, one-dimensional, 'commonsense' definitions like this greatly understate the true nature and complexity of these phenomena. Such lay theorisations are therefore of limited value when workplace practitioners try to make explicit how and why a particular real world interactional problem might have arisen or could be resolved.

This brings us to the question in the title of this chapter. This was posed by a slightly exasperated participant in the course of an action-learning programme, ${ }^{1}$ which involved a structured process of critical reflection on a series of work interactions nominated by himself or others as being problematic for one reason or another. His practical engagement in this dialogue had led him to realise that there was no simple and unambiguous answer to his question. Previously unrecognised issues, dimensions and contributing factors had been identified in the focus interactions, many of which could be interpreted from different perspectives; these additional

\footnotetext{
${ }^{1}$ This was one of the pilot action research programmes involving critical reflection on participants' own workplace communication discussed in the previous chapter, and took place over a period of several weeks. See also further discussion later in this chapter.
} 
layers of complexity also meant there were no 'quick fixes' to the kinds of communication problems he and his colleagues wanted to address.

The outcome of this practical workplace communication exercise interestingly mirrors the heterogeneity of existing theoretical perspectives, and further reinforces the conclusion that miscommunication and problematic talk are indeed very slippery and multi-layered concepts. This example also suggests that many of the same issues that have proved so complex to resolve from a theoretical perspective, are also likely to arise for practitioners in applied contexts when they systematically explore the incidents of miscommunicative talk in which they are involved.

In practice, of course, people seldom consciously analyse their own talk in any detail, especially not at the level of an individual utterance or interaction, and there is a tendency for people to assume the accuracy of commonly articulated lay conceptualisations of miscommunication, and/or to underestimate the amount of interactional troubleshooting they routinely engage in. These lay impressions are not in fact reflective of what actually happens: on the contrary, in the data from this study, participants could be routinely observed to orient to, respond to and work at pre-empting or repairing problematic issues as these unfolded during talk-ininteraction or across related interactions, often operating at a number of different levels, and simultaneously attending to multiple meanings and interpretations.

Careful analysis of everyday workplace talk also frequently shows people taking preemptive action to prevent interactional trouble or communication breakdown, and to manage potentially face-threatening or conflictual situations before they can pose a threat to longer-term working relationships. In most cases of naturally occurring interaction, much of this intricate footwork happens at a largely unconscious level for the people involved as interactions unfold and intersect with one another 'on line' and in 'real time'.

Even the very brief data excerpt reproduced at the beginning of this chapter provides a rich source of evidence in support of this conclusion. In this extract from a much longer interaction, Jan appears to orient unambiguously to the commonsense lay meaning of miscommunication as she seeks to explain a recent workflow problem that has arisen within her department. On closer examination, however, this apparently straightforward example also reveals unexpected layers of complexity. 
This particular exchange comes towards the end of a lengthy meeting between Jan, who is a senior manager, and Heke, ${ }^{2}$ one of the team leaders in her section. The meeting has focussed on a number of misunderstandings and differing work expectations within Heke's team, and has been somewhat tense at times up to this point. In the first line of the excerpt, Jan sums up her instructions for resolving the last of these problems, along with a justification for proceeding in this way. Heke acknowledges Jan's directive in the first part of his succeeding turn with all right okay, but then shifts to a new action, an apology, which shows he has oriented to the second part of Jan's utterance as a complaint. His apology, initiated with a dramatic sigh, is fulsome and, with her point apparently accepted, Jan's response is to start on a pre-closing sequence in which she plays down the seriousness of the problem and makes it clear she is not holding Heke personally responsible by offering the rationale that there was clearly some miscommunication somewhere.

In this excerpt, a specific (mooted) instance of miscommunication is in explicit focus as the underlying cause of the practical work problem that Jan and Heke have just resolved by means of their current discussion. The substantive problem under discussion itself involved a minor lapse in communication - certain essential information had been omitted from a letter, and the CEO, Philip, had therefore declined to sign it off, causing a delay in a project. At the same time, there is evidence of problematic talk of a rather different kind located within the current interaction itself. Jan's criticism of the performance of Heke's team, and hence, by extension, of his effectiveness as their immediate line manager, means there are a number of tricky relational and identity issues at stake here, all of which, potentially at least, need to be carefully managed interactionally to preserve the parties' longer term working relationship, and their own personal positions within the organisation.

For Heke, both the underlying issue of his team's non-performance and Jan's implicit criticism are potentially face-threatening in terms of his professional reputation as a competent team leader. Jan, on the other hand, indexes her own close working relationship with Philip, the CEO, by the way she uses his first name and is able to report directly what he wanted. This, together with her explicit instruction to Heke to check out ... what happened, foregrounds her status as a senior manager. Such

\footnotetext{
${ }^{2}$ As elsewhere, all names used in transcript excerpts in this chapter are pseudonyms. 'Heke' is a Māori name, pronounced in English as 'Hecky'.
} 
explicit indexing of her managerial authority was unusual for $\mathrm{Jan}^{3}{ }^{3}$ who more typically played down status differences with her subordinates; hence this strategy was interactionally salient here (as indicated by Heke's explicitly apologetic response). However, Jan's non-threatening formulation of her directive, mitigated with the hedge just, along with her alternative account of the cause of the problem both work to balance this assertion of status, arguably reinforcing her identity as a firm but fair manager. In just these few lines, then, we can see Jan and Heke negotiating several problematic aspects of workplace talk: the downstream consequences of the omission of information from a letter, the difference in their status, protecting their personal 'face' and their positions within the organisational hierarchy, while at the same time working to maintain an amicable personal and working relationship.

What makes this example especially interesting is the way in which the lay concept of miscommunication is used as a resource for managing these other tricky issues. Attributing the problem to miscommunication in this way provides Jan with a convenient strategy for tabling her expectation that the issue should not recur, whilst at the same time managing a potential relational difficulty. In addition, Jan's vague formulation, some miscommunication somewhere, indexes the difficulty of locating the precise point at which an instance of problematic talk has occurred, and implies that she considers it unimportant to understand the exact nature and provenance of the supposed miscommunication.

This introductory example succinctly illustrates an important point: namely, that at a fundamental level discourse analysts and workplace participants face similar problems in trying to understand a given instance of miscommunication or problematic talk. The first requirement of course is to recognise that an interactional problem has in fact occurred (or has the potential to unfold). The second is to 'analyse' or understand the issue at stake and how it arose, taking account as far as possible of all relevant dimensions or factors. As we have seen, in the case of workplace practitioners, a third task is to select appropriate communicative strategies for dealing with any problems identified within the dynamic and contingent environment of an ongoing interaction, and to align these strategies to the way in

\footnotetext{
${ }^{3}$ Jan was a key informant at this workplace and recorded 14 of her own interactions over a period of about three weeks.
} 
which their interactant responds in turn and to their longer term transactional and relational goals.

The question in the title and the reflective process that gave rise to it provide a logical starting point for the focus in this chapter on developing a working model for analysing miscommunication and problematic talk in workplace discourse. The next section (5.2) sets out a proposed multi-dimensional analytic framework that aims to draw on the perspectives and meet the needs of both researchers and workplace practitioners. Section 5.3 applies this framework to a thick description of three cases of miscommunication and problematic talk drawn from the baseline data set described in the previous chapter. These illustrative analyses have been selected to identify the kinds of communication problems that typically occurred in the workplaces studied, to unpack some of the issues that arise when we attempt to analyse them, and to describe the strategies used by participants to prevent or repair instances of miscommunication and problematic talk. The final section (5.4) briefly evaluates the strengths and limitations of this analytic framework in terms of how well it has accounted for the complexities seen in the cases analysed, and the degree to which a flexible pluralistic and multi-layered approach such as this might add value to existing tools and approaches.

\subsection{Analytic framework}

\subsubsection{Issues of practical relevance}

The review of the literature in chapter 3 highlighted the diversity of existing theoretical perspectives and methodological approaches to miscommunication and problematic talk. It also identified the definitional and analytic issues that are likely to arise as we begin to explore the 'real world' cases that are at the heart of the present study. This review largely addressed my first research objective, which was to explore the most relevant theoretical approaches to analysing these issues in naturalistic workplace data. It also took us some way towards the second, namely, to identify the specifications for a methodological and analytic framework that takes account of the highly complex intertextual and situated nature of miscommunication and problematic talk in such contexts, and we will return to this objective in the second part of this section. However, to have true "practical relevance" (Roberts 
1997; Roberts and Sarangi 1999), such a model needs to go beyond providing a purely theoretical framework for application by researchers, to accommodate a degree of "joint problematisation" (Roberts and Sarangi 1999: 473). In keeping with the third objective of this study, then, it must also provide an integrated framework and set of analytic tools that will allow workplace communication researchers and practitioners to "think practically and look locally" (Eckert and McConnell-Ginet 1992) as they attempt to understand and deal with actual instances of miscommunication and problematic talk.

In my own experience over the years, both as an interactional sociolinguist and as a practicing workplace communication consultant, the depth and breadth of the different perspectives and analytic tools available has provided a rich and eclectic resource on which I have drawn freely and selectively according to the particular research question or practical problem being addressed at the time (and inevitably also on the basis of my own epistemological orientation and areas of expertise). As discussed in chapter 2, this approach is not at all uncommon among discourse analysts and other applied linguists as a way of avoiding a reductionist approach to 'real world' problems. At the same time, the diversity and lack of coherence in existing models and approaches to problematic discourse has at times been a source of intellectual confusion and conflict as I have sought to reconcile the tension between the need to take a theoretically rigorous and clearly focused approach to my work, whilst also wanting to take advantage of the insights afforded by admitting multiple perspectives and complementary, open-ended readings of the same data (cf. Stubbe et al 2003, Weatherall, Stubbe et al in press).

From a practical perspective, the context of the problem at hand is crucial in deciding which aspect of workplace miscommunication it is important for participants or analysts to focus on at a given time, as we saw in the second part of chapter 3. For an airline pilot trainer or crash investigator, for example, the immediate context of the talk and a fairly microscopic approach to how and why any misunderstanding might have arisen is not only appropriate but essential, although at some point, systems factors might also come into focus in such a case. For a manager in an organisation faced with conflict or other dysfunctional patterns of communication, the immediate focus is likely to be at a different level of detail. In such a context, the most useful model is likely to be one that focuses on intergroup relationships and/or on how 
communication becomes distorted (or not) over time or across a distributed genre network or web of interactions.

Of course researchers and workplace participants also operate from rather different perspectives, and have different resources at their disposal. Like consultants, discourse analysts are usually 'outsiders' who can provide a different viewpoint to that of the workplace practitioner and offer insights into patterns of interaction and systems of communication that may be invisible to the workplace practitioners themselves. Analysts may also have access to recorded data or systematic observations which allow more direct analysis than is available to workplace participants who usually rely on informal observations or retrospective analyses based on recall, the reports of others or written summaries. The outside analyst can also potentially add value by deconstructing an interaction at a fairly microscopic level of detail (as above) to reveal the complexity of what is happening turn by turn in a given interaction or other kind of communicative sequence, and can apply a range of more or less sophisticated theoretical models and analytic tools to this task. On the other hand, as insiders, workplace participants have the advantage over outside analysts in terms of their in-depth contextual knowledge and their ability to ground a particular interaction in the wider discursive fabric of the workplace. A research recording generally represents only the tip of the iceberg, a 'snapshot' of one tiny aspect of the complex web of interactions from which it is drawn. This means the most constructive approach is one which draws on both sets of perspectives and sources of data and interpretation.

For these reasons, the analytic framework developed for this study explicitly draws on and complements the communication evaluation and development (CED) model that was originally designed and trialled with workplace participants as part of the LWP project (see Jones 2001, 2002; Jones and Stubbe 2004). Rather it developed in response to requests from research participants for a structured way to incorporate the research process and its findings into their own workplace practices on an ongoing basis.

The CED model operates in the context of participants' own interactional practices, facilitating open-ended cycles of structured observation and action learning. As such it explicitly acknowledges the dynamic and situated nature of workplace communication processes. As briefly discussed in chapter 4 , it is underpinned by the 
principles of reflexive learning, appreciative inquiry and participatory action research (see Fig 4.1) When incorporated into an action research paradigm, this approach also provides both workplace communication researchers and consultants with a unique way of gaining deeper insight into the culture and context of a particular worksite, and this additional form of ethnographic information has been drawn on as relevant to the analyses presented in this and the two subsequent chapters.

The CED model provides a set of straightforward heuristic tools for helping individuals or work teams identify what is actually going on in relevant interactions, checking their perceptions and expectations with those of others, and allowing them to consider what steps they need to take to improve communication from their own perspective. The model is based on the two key elements of an action learning approach, namely, evaluation (developing insights from reflection on past events and observation of current practices) and planning (applying these insights to future actions). There are four steps in the action-reflection process used in the CED model:

1. Identify a problem/development issue to be addressed

2. Analyse what happened in a specific instance of communication involving the relevant issue

3. Reflect on ways in which things might have been done differently

4. Experiment with ways of approaching future interactions differently.

This approach has obvious relevance for analysing communication problems and trying different strategies for resolving these. For example, one manager participating in the pilot used it as a tool to help work out why his staff were consistently misinterpreting the urgency of assigned tasks. Alternatively, post-hoc reflection on the causes of a misunderstanding or on habitual interaction patterns can serve as a valuable first step in defusing tension, as useful preparation for avoiding a repetition of miscommunication, or as a tool for better understanding and addressing a wider inter-group agenda or dysfunction. An example of the latter was provided in an interview by a Māori policy advisor from another organisation who was concerned about the communicative difficulties faced by Māori participants in officials' meetings. He commented on what he had learned from reflecting on recordings collected in his workplace as follows: 
For an indigenous people, to have access to that type of knowledge and skills when we're in those type of forums so that we can actually get our messages across, so they are actually heard, would be a very powerful tool for us, because we are continually misheard, misrepresented, misinterpreted... and from my experience, I just come home absolutely exhausted, I just feel like I've been sucked dry.

Sometimes, using a structured reflection process also allows people to re-evaluate something more positively that they have earlier considered problematic. In another case (discussed later in this chapter), two senior women meeting to complete a particular task initially assessed their meeting as being unproductive and full of irrelevant digressions. On reflection, and after further analysis, they found that almost all the so-called digressions had served a valuable purpose, though not necessarily in relation to the immediate problem they were currently discussing.

The CED model is also useful when applied more holistically to communication within whole teams to analyse systemic patterns and issues in addition to more localised communication problems. For instance, the managers of the factory production team described later in this thesis, worked with the researchers to adapt and apply the model to an evaluation of this team's communication on the shop floor. This was a high-stakes environment where miscommunication was a constant risk (see chapters 6 and 7). The action research model made it possible to identify those elements of the team's discursive practice that contributed positively to their performance, and to build on these practices to create a more resilient and effective communication culture.

The great advantage of this approach is its potential for sensitivity to the complex and specific contextual factors identified throughout this thesis as being crucial in accounting for the dynamics of interaction in general, and miscommunication and problematic talk in particular. Developing the ability to observe, analyse and reflect on the communicative challenges in one's own particular work environment is far more empowering than the traditional pattern of training in workplace communication based on narrow and prescriptive skills or competency-based approaches. However, to be most useful, such an approach ideally needs to incorporate a theoretically robust but user-friendly framework for analysing the issues under consideration. A proposed working model for such a framework is presented next. 


\subsubsection{Analytic process and dimensions}

\section{Underlying theoretical principles}

The analytic framework presented here grew organically out of the insights gained by looking across a large set of workplace interaction data over time as part of an ongoing iterative process. This involved analysing and reflecting on many specific interactional sequences, as well as examining a number of more extended and complex cases which were identified as potential examples of problematic communication within the baseline data set described in chapter 4 (see criteria below). The development of this 'working model' thus followed a conventional datadriven analytic pathway in many respects, but often it was serendipitous observations triggered by dialogue and engagement with the workplace participants themselves which proved to be the most immediately interesting and revealing. These forced me at various points to reconsider previously taken-for-granted concepts and approaches as I grappled with questions such as how best to identify and define miscommunication and problematic talk in the data, what kinds of factors and dimensions needed to be accounted for, and which models and tools I could use myself and/or offer to the research participants to analyse these cases.

The theoretical assumptions and methodological approaches that underpin the development of the analytic framework proposed here have already been thoroughly canvassed in chapter 4 . To recap briefly, the basic paradigm adopted here is a social constructionist one. Communication and miscommunication are assumed to be coconstructed dynamic interactive processes within which social identities and group boundaries are constantly being created and (re)contextualised. Specific interactions and genres thus do not stand alone, but are produced within a network or complex adaptive system characterised by intertextuality and interdiscursivity.

The analytic methodology draws on the insights and approaches of different theoretical and descriptive models of miscommunication within a unifying framework of critical interactional sociolinguistics. Taking this approach accommodates the application of fine-grained interactional analysis within a broader ethnographic and socio-cultural context; it also facilitates a focus on the interactional practices and processes people can be observed to engage in as they negotiate their way through the complexities of workplace discourse and relationships. 
The objective was not to produce a 'grand theory' of miscommunication, but rather, to respond to the call by Coupland, Wiemann and Giles to undertake "further empirical studies and integrative efforts" with the aim of contributing to the ways in which miscommunication "can profitably be understood by interactants and researchers alike" (1991:17). However, designing an integrated heuristic framework for analysing real world instances of miscommunication and problematic talk that is at once theoretically robust and meaningful to the participants themselves presents quite a challenge.

As we have already seen, there is great diversity in the theoretical approaches available for investigating these phenomena, each of which defines them in different ways, and teases out different aspects. It is also possible to analyse such cases at many different levels from the perspective of the participants themselves. This diversity of perspectives reflects the degree of genuine complexity involved in any analysis of miscommunicative discourse. This is a particular challenge for researchers or practitioners who are interested mainly in the situated examination of whole cases or episodes, by contrast with the more abstract 'thin-sliced' deconstruction of very specific aspects that is found in much of the theoretical literature. The underlying rationale for the analytic framework proposed here, therefore, is that it should be sufficiently flexible and spacious to allow the rigorous application of a range of relevant tools and perspectives to the messy, complex reality of actual cases of problematic communication in workplaces.

Table 5.1 provides a summary overview of the working model for identifying and analysing instances of miscommunication and problematic talk that has developed out of the process and principles outlined above. This working model is intended to function as a heuristic framework to assist with teasing out the many possible overlapping dimensions and levels of analysis that need to be considered, not as a rigid prescription for a stepwise analytic process.

The left hand side of the summary table lists six analytic actions which have been arranged in a logical but not necessarily chronological sequence. The bi-directional arrows thus indicate that there may be multiple entry points for the processes of identification and analysis, and are also reflective of the dynamic, iterative and overlapping nature of these analytic actions. 
The right hand side of the table lists six sets of 'analytic dimensions or levels' which correspond to each 'analytic action' on the left (scan, describe, define, locate, diagnose and evaluate). In addition, the rightmost column identifies seven further analytic dimensions which are 'omni-relevant' ${ }^{4}$ in the sense that they have global significance and may enter the analysis at any point.

The remainder of this section briefly defines and explains the scope of each of the main components of this analytic framework, which will be further exemplified as it is applied to the illustrative case studies that follow.

\section{'Candidate episodes'}

Defining precisely what 'counts' as miscommunication or problematic discourse and how the relevant phenomena should be categorised in relation to one another is no straightforward task. Miscommunication and problematic talk are complex, dynamic and multi-faceted processes, not usually static and clearly bounded events. Moreover, because miscommunication is in fact part of the normal give and take of human interaction, it can also be difficult to draw the line between miscommunication and the normal processes of negotiating meaning, especially if the latter involves an element of misalignment or some kind of threat to face such as a disagreement, complaint or directive, rather than an obvious linguistic or pragmatic ambiguity or misunderstanding as such.

In the workplace setting especially, people are constantly engaged in a delicate discursive balancing act as they attempt to get the job done, whilst at the same time managing their relationships with their co-workers, managers and others with whom they interact on a daily basis. It is the tensions between these sometimes conflicting roles and imperatives that often render workplace talk problematic, rather than features of the interactions themselves. Recognising and classifying these phenomena when they do occur, and determining precisely where they are located, are equally complex and problematic issues. As a result, it is not always easy to identify an instance of problematic talk or miscommunication as such, either for the analyst or for participants themselves.

\footnotetext{
${ }^{4}$ This term has been borrowed from the ethnomethodological concept of the "omnirelevance" of certain contextual variables such as gender (Garfinkel 1967:118).
} 
Table 5.1: Analytic framework

\section{Candidate episode of miscommunication or problematic talk}

\section{Analytic dimensions or levels*}

Nature of evidence

\section{Analytic action}

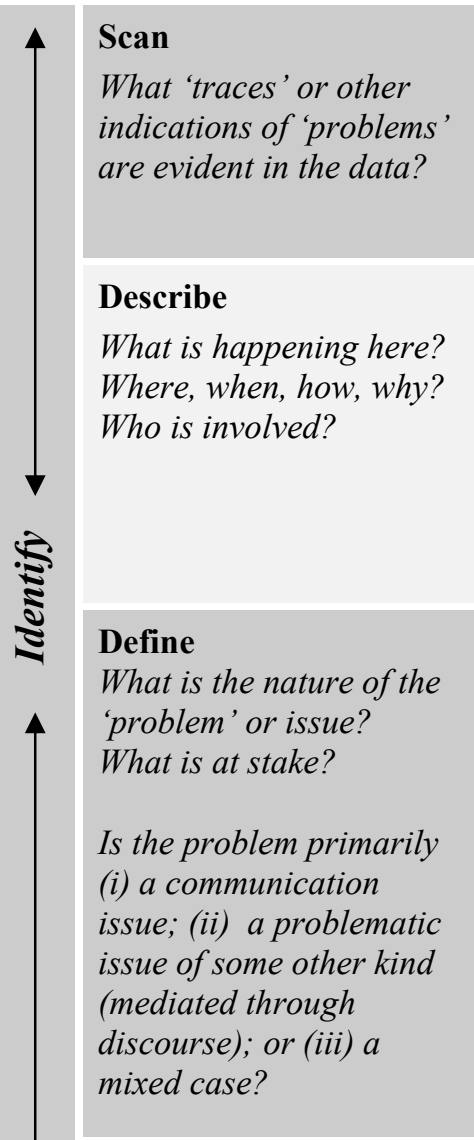

\section{Locate}

Where does the observed episode 'fit (in time and sequentially)? Does it have defined boundaries, and what is its extent?

\section{Diagnose}

What is the underlying cause or origin of the problem?

Is it possible to identify a source or trigger?

\section{Evaluate}

What are the likely outcomes (actual or potential), and how consequential are they? What strategies have been/could be used (if any) to mitigate and/or resolve the matter?
Interactional: observable issue or 'trouble' (microanalytic features, immediate context)

Intertextual: 'thread' to or from linked interactions Ethnographic: clue(s) from historical or background information (observations, reports, documents)

'Situation': setting, time, context

'Participants': individuals/relationships

'Ends': goals and outcomes - interactional/concrete

'Actions/activity sequences': content/design/structure

'Keys': tone/manner, footing

'Instrumentalities': channel, media, linguistic code/style 'Norms': interactional and interpretative 'rules'/practices 'Genres': types, sets, networks/systems

Referential: Information content, knowledge

Instrumental: Goals and outcomes

Affective: Perceived intent, attitudes

Relational: Power, solidarity, identity, face

\section{Discourse-internal}

Interactional incident or process at one or more levels (non-verbal, paralinguistic, pragmatics, turn design, sequential organisation, coherence, genre, linguistic code)

Contextual *2:18)
Mismatches or gaps in
background knowledge or
understandings
Aspect of setting or
situation (e.g. work
environment, systemic
issues, a practical or
interpersonal problem)

Localised: $a$ 'focused' one-off incident which originates, develops and is resolved within a single sequence or interaction

Global: an 'unfocused' problem, characteristic of $\quad * 2: 190$ a whole sequence, interaction, or a series of encounters Multiplex: one of a series of episodes in a longer sequence/process, or a surfacing or 'trace' of a past event

1: Flawed 'transfer' or negotiation of meaning $*^{3}$ » localised breakdowns » minor misunderstandings » misreading of intentions or attitude 'Not getting the message (across)'

2 Goal conflict/ failure to achieve goals $*^{3}$

» resistance » non-alignment »'strategic' miscommunication (misleading, withholding, masking) 'Talking at cross-purposes'

3 Differing group/sociocultural norms/practices $* 3$ »'crosstalk' (misalignment, misunderstandings, misattribution) » exclusion » discrimination/inequalities 'Talking past each other'

4 Competing/hegemonic discourses or ideologies $*^{3}$ » repressive discourse, masking » coercion » silencing » conflict » marginalisation/exclusion 'Conflict or disempowerment'
* A number of the analytic concepts and terms used in this framework have been adapted from previous models (as marked) ${ }^{* 1}$ Hymes (1974): 'SPEAKING' grid; ${ }^{2}$ Linell (1995:188-90); ${ }^{3}$ Coupland, Wiemann and Giles (1991): 'integrative model' 
The remainder of this section briefly defines and explains the scope of each of the main components of this analytic framework, which will be further exemplified as it is applied to the illustrative case studies that follow.

\section{'Candidate episodes'}

Defining precisely what 'counts' as miscommunication or problematic discourse and how the relevant phenomena should be categorised in relation to one another is no straightforward task. Miscommunication and problematic talk are complex, dynamic and multi-faceted processes, not usually static and clearly bounded events. Moreover, because miscommunication is in fact part of the normal give and take of human interaction, it can also be difficult to draw the line between miscommunication and the normal processes of negotiating meaning, especially if the latter involves an element of misalignment or some kind of threat to face such as a disagreement, complaint or directive, rather than an obvious linguistic or pragmatic ambiguity or misunderstanding as such.

In the workplace setting especially, people are constantly engaged in a delicate discursive balancing act as they attempt to get the job done, whilst at the same time managing their relationships with their co-workers, managers and others with whom they interact on a daily basis. It is the tensions between these sometimes conflicting roles and imperatives that often render workplace talk problematic, rather than features of the interactions themselves. Recognising and classifying these phenomena when they do occur, and determining precisely where they are located, are equally complex and problematic issues. As a result, it is not always easy to identify an instance of problematic talk or miscommunication as such, either for the analyst or for participants themselves.

The scope of the analytic framework presented here is therefore deliberately broad and inclusive in terms of the criteria for identifying 'candidate episodes' or cases of miscommunication and problematic talk. These may include actual breakdowns in the joint negotiation of meaning at some level, as well as cases where participants demonstrably orient to a current, past or potential occurrence at a transactional or interpersonal level. It may be clear from the discourse itself that participants themselves are orienting to some aspect of problematic communication, or there may be other evidence from related interactions or from ethnographic information which identifies a particular interaction or set of interactions as being problematic. 
Instances found in the baseline data set also ranged from superficially straightforward and localised misunderstandings to more complex and extended instances of problematic discourse relating to the ongoing negotiation of workplace tasks, processes and relationships.

\section{Omni-relevant dimensions of analysis}

There are several dimensions of analysis which are potentially relevant at different points in the analytic process and therefore need to be considered as potential global factors during any or all of the initial identification, definition/classification, and 'diagnosis' or evaluation of an episode of miscommunication or problematic talk. These omni-relevant analytic dimensions are discussed in three groups: 1. awareness and repairability; 2. intentionality, perspective and agency; and 3. multi-functionality and intertextuality. ${ }^{5}$

\section{Awareness and repairability}

Miscommunication often goes unrecognised by all or some parties to it, and some instances will remain invisible to both analysts and participants. As a result, seemingly mundane or unproblematic sequences of events or interactions can be "deceptively adequate" (Coupland, Wiemann and Giles 1991), with no overt sign of miscommunication because the participants are unaware they have misunderstood one another. Alternatively, many actual or potential misunderstandings occur or are resolved with little or no explicit attention paid to them, or the people involved may simply appear to 'agree to disagree'. Linell (1995:187) calls these "latent" and "covert miscommunication events" respectively. He suggests that participants may be aware of "covert" cases, as evidenced by post hoc interviews for example, but that these would mostly correspond to "uncomfortable moments" rather than being "thematised in the discourse itself". "Latent" cases might include inadvertent cross talk between people from different socio-cultural backgrounds where, although analysts may be able to discern a problem, participants themselves are unlikely to be aware of this, or alternatively, may (mis)attribute a perceived problem to some other factor such as personality, attitude or intention. These categories contrast with

\footnotetext{
${ }^{5}$ These global analytic dimensions were also discussed in chapter 3, and, as indicated there, some of these concepts have been borrowed from Coupland, Wiemann and Giles' (1991) integrative model.
} 
"overt" cases which are clearly reflected in the interactional data, and of which participants are likely to be aware themselves.

The significance of this is that miscommunication can be 'repaired' or resolved by participants only if it is 'noticed' at an interactional level or surfaces in some other way. This means it can also be difficult to know whether to 'count' something as an instance of miscommunication or not, even assuming it is possible to identify it as a candidate case in the first place. These issues contribute to the difficulty of systematic capture noted by previous researchers (e.g. Milroy 1984; Tzanne 2000), and highlight the importance of contextual and 'insider' knowledge in identifying and analysing instances of miscommunication.

\section{Intentionality, agency and perspective}

Even where something can be clearly identified as a case of miscommunication or problematic talk, and there is evidence that one or more participants are aware of this problematicity, it may not always be obvious whether it has occurred purely unintentionally or whether there is a degree of calculation involved. This is more a matter of degree than something that can be determined on a black and white basis, but has obvious implications when it comes to determining why a problem has occurred, who was responsible for it, and/or what strategies might be invoked to address it. It also strikes at the heart of how to define miscommunication in the first place.

As discussed in chapter 3, almost all communication could paradoxically be classed as 'miscommunicative', because effective communication at one level often relies on or risks miscommunication at another. Thus people may engage in "strategic ambiguity" or deliberate (apparent) misinterpretation as one element of face work (Tzanne 2000), rather than being maximally clear and explicit, or they may strategically attribute some other problem (e.g. non-cooperation, a mistake, an interpersonal issue) to miscommunication, as we saw in the opening example to this chapter.

Both these issues relate closely to the dimensions of agency and perspective. As in the example quoted in the title of this thesis "was that my misunderstanding?", it is often important for people to reach agreement on who is responsible and to understand how the different parties perceive it. This also raises some tricky 
definitional issues. For example, should we count something as a miscommunication if one person intentionally misleads another, thereby achieving a particular goal, while the other person remains unaware that this has occurred?

\section{Multi-functionality and intertextuality}

A closely related issue is the fact that utterances and discourse strategies are often multi-functional or open to multiple interpretations, and can index or cross-reference linked events and interactions in complex ways. As we saw very clearly in the example analysed in the introduction to this chapter, communication is much more than a simple, linear flow of information. This once again highlights the theme which recurs throughout this thesis, namely, that any analysis of problematic talk must take account of the wider context and the intertextual nature of workplace discourse and indeed relationships - it is seldom adequate to look at a single interaction in isolation.

\section{Analytic actions and corresponding analytic dimensions or levels}

Table 5.1 lists six analytic actions (scan, describe, define, locate, diagnose, evaluate) along with a corresponding set of analytic dimensions or levels to consider in the identification and deconstruction of a candidate episode of miscommunication or problematic talk. The latter are drawn (in adapted form) from various sources, as indicated in the table, and represent a synthesis of various descriptive analytic tools or heuristic frameworks from the existing literature that have proved useful in communication evaluation and development work with research participants and other workplace practitioners. As noted above, the order in which these are listed is not intended to suggest a predetermined analytic sequence.

\section{1. $\underline{\text { Scan }}$}

Scanning for candidate episodes of miscommunication or problematic talk may take place consciously or unconsciously 'on line' and in 'real time', or it may be a post hoc reflective or analytic activity. In either case, for an analyst or participant to identify that a problem has definitely occurred (or for them to suspect as Jan did that "there must have been some miscommunication somewhere"), there has to be some kind of interactional trace or other contextual indication of a 'problem'. This evidence might be interactional (something that is directly observable or oriented to interactionally); it could be intertextual (something which arises or is commented on in a subsequent interaction) thus providing a thread or link across contexts; or it may 
be gleaned from ethnographic information (observations, reports or interviews, historical or background information) which can alert the practitioner or analyst to examine any relevant interactions, if these have been recorded.

In this sense, analysts and participants face similar issues. Unless or until a communication problem actually surfaces, it is not possible to identify or analyse it, and even then it may only be identifiable by inference from some kind of negative outcome or inexplicit clue, interactional 'trouble' or discomfort. Conversely, the fact that a particular 'bit' of interaction is problematic and/or might lead to problems down the line cannot necessarily be predicted at the time.

\section{Describe}

Before undertaking a detailed interpretative or critical analysis of an identified instance of miscommunication or problematic talk, it is generally useful to undertake a systematic sociolinguistic and ethnographic description or neutral 'denotative analysis' (Baxter 2003) of the sequence or episode in question to establish its basic 'narrative' and contextual elements and structure (i.e. 'what is happening here?'). This can also be a useful component of 'unmotivated' systematic observation. ${ }^{6}$ Hymes' (1974) well-known "SPEAKING" grid has proved to be a useful and accessible framework to use for these purposes with workplace participants in action learning settings, but other similar frameworks would serve just as well.

\section{Define}

The action of defining as presented here operates at a more detailed level of analysis than the initial identification of a candidate episode, and seeks to delineate what 'matters' or issues can be inferred or observed to be at stake (i.e. what is the nub or focus of the problem?) - and the nature or type of miscommunication or problematic talk that is involved. Two different sets of analytic dimensions are applicable here. The first set is based on the standard sociolinguistic/pragmatic 'levels' of meaning, and classifies the focus of the problem according to whether its nature is primarily referential or propositional (accuracy or content/knowledge), instrumental (participants' goals or intended outcomes), affective (perceived intent or attitudes), or relational (power, solidarity, identity or face).

\footnotetext{
${ }^{6}$ This term has been borrowed from the slightly different conversation analytic construct of "unmotivated looking" (ten Have 2007) which refers to the CA practice of examining sequences of interaction in a collection of data without a pre-determined analytic agenda.
} 
The second set of dimensions relates to whether the problem is primarily (i) a communication issue; (ii) a problematic issue of some other kind (mediated through discourse); or (iii) a mixed case. Here I have borrowed some terminology from Linell (1995) who distinguishes between "miscommunication events" that are "discourseinternal" versus "contextual" in nature (188). Because my analytic framework is not limited to consideration of localised sequences as Linell's is, I have also added a third category, the "mixed case". For my purposes here, "discourse-internal" cases are those where a problematic interactional incident or process can be clearly identified at one or more communicative or linguistic levels (including non-verbal, paralinguistic, pragmatics, turn design, sequential organisation, coherence, genre, and linguistic variety or code). "Contextual" cases could include mismatches or gaps in background knowledge or shared understandings, or aspects of the setting or situation (including the physical work environment, systems issues, and practical or interpersonal problems). Mixed cases may involve elements of both, as for example in the opening excerpt where there is a current set of "discourse-internal" issues which are directly observable in the interaction, but which do however relate directly back to a "contextual" problem, namely the lapse on the part of Heke's team.

Essentially this latter categorisation amounts to a distinction between miscommunication/problematic talk as such, and talk that is about actual real world problems (or is 'difficult' essentially because of these). In practice, the definitional boundaries are not always easy to draw for either set of dimensions, whether analytically or for participants, as they are inclined to be rather fuzzy, and as mentioned above, many utterances and situations are open to multiple interpretations, often simultaneously.

\section{Locate}

Another important analytic action is to locate, as far as possible, where the observed episode fits into the overall context, in time and sequentially or intertextually, whether it has defined boundaries, and what its extent or scope is. The concepts and terms for the first two categories, "localised" and "global" events, are again borrowed from Linell (1995: 190), but adapted here once more to apply to series or networks of interactions as well as to single sequences at the local level of talk-ininteraction. "Localised" episodes are those where a "focused" one-off incident originates, develops and is resolved within a single activity sequence or interaction. 
"Global" cases involve an "unfocused" problem, which may be characteristic of a whole sequence, interaction or series of encounters. Multiplex cases are those based in one of an identifiable series of episodes in a longer sequence or process, or where there is a surfacing or trace of a past event in an interaction.

\section{Diagnose (causes) \\ 6. Evaluate (outcomes)}

The final two analytic actions, 'diagnose' and 'evaluate', are dealt with together here for convenience, as the corresponding analytic dimensions or levels for both consist of an adapted version of the Coupland, Wiemann and Giles (1991) integrative levels model; in practice however, it is often helpful to separate these out analytically (see below). The action 'diagnose' seeks to identify the underlying cause(s) or origin(s) of the problem or issue at stake. Even where it is not possible to identify a specific source or trigger, it is often possible to determine the likely generic level at which the problem lies (bearing in mind the discussion in chapter 3 where it was pointed out that more than one level may apply at a time). 'Evaluation' involves analysing what the actual or likely outcomes or consequences are or might be of a given episode of miscommunication or problematic talk, assessing how serious these might be in practical, relational or communicative terms, and identifying the strategies that have been/could be used (if any) to mitigate and/or resolve the matter.

There are four analytic dimensions or levels relevant to the diagnosis of underlying cause(s) and evaluation of outcome(s); as already noted, these represent a reworked version of the Coupland et al (1991) integrative model. The first category covers problems arising from 'hitches and glitches' in the actual process of communication. Localised outcomes could include infelicities or breakdowns in the transfer or negotiation of meaning, minor misunderstandings or misreading of intentions or attitudes, and/or practical consequences of various kinds. I have glossed this in lay terms as 'not getting the message (across)'. Possible repair or mitigation strategies might include such things as redundancy and repetition, clarification requests and interactional feedback, and the use of addressee and speaker-oriented pragmatic devices.

The second category is glossed as 'talking at cross-purposes', and comprises issues arising from conflicting goals or a failure to achieve a desired outcome at an interpersonal level. Interactionally, this might result in resistance and non-alignment, 
or various kinds of deliberate or strategic miscommunication. Interactional repair and mitigation are likely to be achieved by means of a range of pragmatic and linguistic politeness strategies.

In the third category, the causal factors relate to differing group or sociocultural norms and practices and are glossed as 'talking past each other'. Possible consequences include various kinds of 'crosstalk' such as misalignment, misunderstandings and misattribution, and also intentional or unintentional discrimination and inequalities. Interactional features or strategies are again likely to involve localised pragmatic and linguistic practices, as well as the development of intergroup and intercultural awareness at a more abstract level.

Problematic talk in the fourth and final category is rooted in conflicting societal discourses or ideologies. Issues at this level are likely to play themselves out in the form of repressive or oppressive discourse, masking, coercion, silencing, and exclusion, along with overt and covert resistance and can be expressed in lay terms as 'conflict or disempowerment'.

Returning to the point alluded to above, it is important for analytic purposes to separate out 'trigger' events (usually some kind of misunderstanding or disliked/unwanted (in)action, or overt conflict or tension), from any subsequent negative consequences which have surfaced, including further problematic communication. As already noted, the analysis of the opening excerpt provides a good example of the need to be clear about exactly what the analytic focus is at any given point. This example also highlighted another common scenario, in that by the time negative outcomes occur or traces of a previous miscommunication surface (if they do), it is often difficult or impossible to pinpoint the precise source of the problem. However, for participants, this is the point at which it will need to be dealt with, and it is here that we often see the kind of intricate verbal manoeuvring and 'damage control' that Jan and Heke engaged in.

This interface between cause and effect creates some of the most interesting and challenging problems for the analyst too, and, of all the analytic dimensions, reveals most clearly the dynamic and intertextual nature of miscommunication and problematic talk, and the reason why it can be so difficult to 'capture' instances for analysis. The interdependencies are complex and unpredictable. A trigger event may function something like a stone dropped into a pond, so a small misunderstanding or 
framing error can easily escalate or compound. Alternatively or as well, it may affect how subsequent utterances or behaviours are (mis)understood or (mis)interpreted, and/or these subsequent misunderstandings may also serve to reinforce the initial one in turn, thus creating a faulty logic chain. Such complex scenarios are very common. They are at the heart of many workplace disputes, disagreements and relationship difficulties. However, it can be very difficult indeed, if not impossible, to unravel them, particularly when it is unclear whether the episode that has come to our attention as analysts or as participants is like the stone at the centre, part of a wider ripple effect from something else, or a complicated interweaving of a number of causal factors.

In section 5.3, this analytic framework is applied to several cases of miscommunication and problematic talk drawn from the baseline data set described in the previous chapter. These illustrative analyses will identify the kinds of issues that typically arose in the workplaces studied, and, where applicable, describe the strategies used by participants to prevent or repair these instances of miscommunication and problematic talk.

\subsection{Illustrative data analysis}

Three cases have been selected for detailed analysis using a 'critical incident' or exemplar approach. This involves closely examining all the information 'to hand' about a particular episode to produce a thick description of what seems to be involved. The aim is to determine what insights might be gained into a particular situation and into miscommunication and problematic talk more generally, by applying different analytic 'frames' or 'lenses' based on the elements of the heuristic framework described above. Where possible, a particular issue or problem has been traced through a range of data sources, which might include recorded interactional data, ethnographic information, or comments from participants in feedback or action learning sessions. The analyses also build on other known information about the workplaces in question or the wider social context, and on related analyses of less problematic interactions from the baseline data set that offer some insight into the strategies used routinely in these workplaces to negotiate relationships and tasks. The 
aim is to test the model by taking the baseline data as far as the limitations of the sample and the methods of its collection will allow.

\subsubsection{Case 1: 'Was that my misunderstanding?'}

This first case takes us back to an earlier point in the meeting between Jan and Heke, a short excerpt from which was analysed in the introduction to this chapter. As already indicated, at the time of recording, Jan was a senior manager in a relatively small but growing government organisation where the staff were under a lot of pressure to perform in a context of multiple complex work streams and high staff turnover. At this time, there was a government-wide policy emphasis on issues such as biculturalism ${ }^{8}$, ethnic and gender equity and work-life balance, and public service organisations were expected to act as role models in these regards. The ethnographic data indicated that equity issues were also currently contentious in this maledominated organisation, where gender equity was perceived by some as cutting across traditional Māori cultural values. Jan was a Pakeha woman with a senior management role leading a section with a high proportion of young and relatively inexperienced Māori staff, and was therefore in a unique and at times somewhat challenging position. Heke was a slightly younger Māori man who had recently been promoted to a team leader position, a role in which he was still finding his feet. He and Jan had regular weekly meetings, one of which provides the focus interaction for this illustrative analysis.

The exchange in (1.1) takes place right at the start of the meeting. There is clear evidence in the data that Jan is orienting to this particular interaction throughout as problematic talk. This is explicitly indexed by her reference to a previous misunderstanding which has had negative consequences, thus triggering the current issue. Heke is still getting himself organised (line 2), but Jan is clearly agitated, as indicated by the abrupt way she begins the discussion, her high pitched delivery, and the way she then immediately issues a challenge what happened to Marama? (line

\footnotetext{
${ }^{7}$ Separate analyses of the two main sections of this interaction presented here have appeared in other publications (Stubbe and Holmes 2000, Holmes and Stubbe 2003a), but they have not previously been analysed as here in an integrated way.

${ }^{8}$ In the New Zealand context, biculturalism refers specifically to Māori-Pakeha relationships as enshrined in the Treaty of Waitangi (Pakeha being the Māori word for New Zealanders of European, especially British, origin). Māori comprise approximately $15 \%$ of the population, and Māori is an official language, but is spoken fluently by only a minority of (mainly Māori) New Zealanders.
} 
4). This occurs without any preamble, before she tables the apparently more conciliatory conclusion that there has been a misunderstanding, leaving open the (notional) possibility that Jan herself may have been to blame for this.

\section{(1.1) Was that my misunderstanding?}

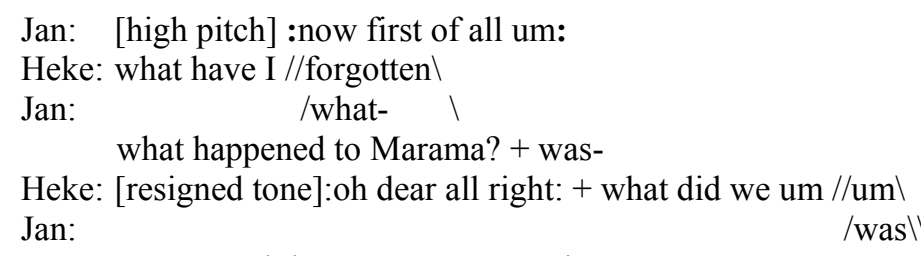

The actual misunderstanding Jan refers to in this example was in itself probably relatively minor, although it is impossible to know this for certain without any additional evidence. What we do know is that Marama had not turned up as expected 
to accompany Jan to a meeting with another organisation, and so Jan as the section manager had been left to contribute to a discussion where she did not have all the information she needed (lines 12-24). There are a number of possible explanations Marama may not have received the information about the meeting, she may not have understood that she was required to attend, she may have forgotten about it or she may simply have failed to ensure Jan was informed that she could not come. It is also theoretically possible, though probably unlikely, that she made a conscious decision not to attend, perhaps due to pressure of other work.

However, whatever the reason, and however minor or deliberate the original misunderstanding may have been, it had serious consequences, first for Jan, and now, by extension for Heke also. Marama's non-appearance at an important meeting with an external stakeholder resulted in embarrassment for Jan, a senior manager, and had the potential to derail some sensitive negotiations. Although Jan gives Marama the benefit of the doubt, was that MY misunderstanding (line 7), it is clear that she nevertheless feels obliged to follow the incident up with Heke who is Marama's line manager. We can infer that this was, in part, to make him aware that a problematic issue had arisen, presumably in the hope of preventing a recurrence, but also to get him to organise some 'damage control' in the form of another meeting and the preparation of a proposal (lines 29-30, 56-58). In doing so, she therefore enters into problematic discourse of a different kind, where the quality of the working relationship between Jan and Heke, and between Jan and other members of Heke's team, is potentially at risk.

On one possible reading, Jan's attribution of the problem to her own misunderstanding may be a genuine 'lay explanation' or hypothesis - an attempt to come to grips with the root causes of a problem. Alternatively, it can be interpreted as a face-saving device, a strategic presumption of innocence. If something is framed as an (unintentional) misunderstanding, this is less serious than admitting to or accusing someone else of making a mistake or, worse, intentionally doing something that has turned out badly. The latter reading seems to be supported by Jan's gently humorous reiteration of her complaint in lines 43-44, prosodically marked by ab audible 'smile voice' and preceded by a mildly self-deprecating disclaimer $I$ presume ... otherwise I've had a complete mental blank.. This is followed by a sympathetic but brief aligning response from Heke, $\mathrm{mm}$ dear me, before he quickly 
turns the subject back to the puzzle of the research topic that had been referred to in the meeting. It seems that Jan is not prepared to leave it at that however; after reaching a consensus on the proposed research project, she closes the topic with a graciously worded reminder in line 54-55, once again produced with an audible smiling prosody but um next time I would appreciate sort of knowing if someone's coming or not. Heke has some difficulty formulating a response, making no fewer than four false starts before he manages to articulate a question as to what is required of him now. This is followed by a more specific set of instructions from Jan in lines 58-61, beginning with can we actually provide them with a proper proposal. The earlier suggestion that the oversight may have been a misunderstanding on Jan's part has now been superseded by a heavily mitigated but nevertheless explicit request that she be informed next time, which strongly suggests the earlier attribution was merely strategic.

The rest of this somewhat lengthy meeting continues in a similar vein, dealing with a number of misunderstandings and differing work expectations in relation to Heke's team. In the final phase of the meeting the same issues are recapitulated during a discussion of the last of these matters. The first excerpt, as already discussed in the introduction, begins with an instruction from Jan aimed at resolving the problem, and once again, with a strategic framing of a possible performance problem as a miscommunication of some kind:

\section{$(1.2)$}

1 Jan: I think that's the best thing it hasn't been signed by Philip because he wanted that information included

2 Heke: all right okay [sighs]: oh look: I'm dreadfully sorry about that

3 Jan: oh well just check out to see what happened +'cause there was clearly some miscommunication somewhere

Excerpt (1.3) shows how Heke responds to this carefully framed directive from Jan.

1 Heke: [quietly]: oh I think they're just taking a holiday from the stress really:

2 Jan: yeah

Heke: but I'm keeping the pressure on [high-pitched laugh] + actually I- I wanted to- get your advice about that I want to do a bit of a wee sort of ra ra speech at the beginning of like of planning day tomorrow we ARE stretched people ARE starting to feel the pressure + but it's it's just the kind of thing you know it's- if if we want to be in the business you're gonna have to live with it you know that kind of thing but I want to say that in such a- I'm starting to really become quite the manager now [laughs] um um + and I don't- er I just I do want to say that- I want to say you know look um you know if we- if we're gonna be good policy advisers and we're wanting to be recognised alongside all the other central ones then 
In this excerpt, Heke seems to interpret Jan's instruction and face saving attribution to miscommunication as further indirect criticism, which he counters by offering at some length to put more pressure on his team to improve their performance, after first symbolically lowering himself in relation to Jan with the statement I wanted to get your advice about that (lines 3-4). In this way, Heke positions himself as a hardworking subordinate who is trying sincerely to meet Jan's needs and respects her greater experience. At the same time he claims solidarity as a capable new manager who is prepared to be tough with his own team when necessary: I'm starting to really become quite the manager now (line 9). He uses a large number of hedging devices in a short period of time, sometimes heavily clustered e.g.: a bit of a wee sort of ra ra speech (lines 4-5), thus signalling the tentative nature of his suggestions, while at the same time claiming common ground with Jan by means of addressee-oriented devices such as you know that kind of thing (line 8). (Hedging devices are in boldface). Heke's laughter, not just in this excerpt but throughout the whole interaction, is at times produced with a rather high pitched marked prosodic contour typical of Māori men in New Zealand English. In this context it may be a sign of embarrassment, as well as indexing Heke's own response to his own semifacetious humorous comments which seem to be a strategy for attempting to keep the interaction informal and relaxed.

It is noticeable that Jan does not interject at all throughout this extended turn, even in the form of minimal feedback or similar response tokens. This lack of alignment or uptake by Jan when Heke pauses in line 6 may be a factor in his renewed elaboration on the same theme, including an escalation of what he is going to demand, or it may simply be that she is allowing him the floor for an extended narrative on what he is proposing to say to his team.

Either way, in excerpt (1.4), Jan eventually breaks into Heke's monologue to suggest a less 'full-on' approach. She appears to be taking great care not to sound critical. She opens with a hedged disagreement marker although, expressed in mildly doubtful tone which suggests she is coaching or suggesting rather than arguing. Her rejoinder in lines 2-3 don't say that as something that should be the norm is very heavily mitigated, and in the remainder of this excerpt, her speech is 
also peppered with hedges like sort of, like, sort of stuff, and the addressee-oriented device you know, mirroring Heke's style in the previous turn. She adopts a conciliatory tone, which is reinforced by using a higher pitch, often associated with a 'feminine' ameliorative positive politeness style, and by lexical echoing in line 7 which signals her acceptance of Heke's proffered sentence completion from time to time in line 6.

1 Jan: [doubtful tone]:although: [syllable-timed delivery]: I mean I can appreciate

2 the- that sort of message but on the other hand um + don't sort of + sort of say

3 that as something that sh- that should be the norm //like \that's really you know:

4 Heke: $/ \mathrm{mm} \backslash$

5 Jan: [syllable-timed delivery]: when thi//ngs are really- : \

6 Heke: /from time to timell=

7 Jan: = [syllable-timed delivery]: from time to time that it's not a goo::d wa::y: of them

8 expecting to organise their work all the time:

9 Heke: ae ['yes'] yeah

$10 \mathrm{Jan}$ : that they need you know it's the old work smarter sort of stuff

11 Heke: yeah

12 Jan: and we need to- to sort of be aware of we being a (friend-) [smile voice]:family

13

friendly workplace .......

As Jan becomes more conciliatory, her speech rhythms become noticeably more syllable-timed. This may be an accommodation to what she thinks of as a Māori English speech style (which does tend to reflect the mora-timing speech rrhythms of the Māori language), and as such may function to further reduce the social distance between her and Heke, and to further ameliorate the tough managerial stance she has been conveying throughout the whole meeting. This interpretation is supported by Heke's use of the Māori ae (meaning yes) in response to Jan's carefully worded suggestion. Jan's reference to the need for the organisation to provide a family friendly workplace can also be read as an appeal to the Māori value system, which accords the extended family high priority, as well as reinforcing the importance of this issue more generally. Jan's response thus positions her as a considerate, reasonable and culturally sensitive manager who, while she wants to see good results, does not expect her staff to work too hard or to sacrifice their family lives: it's the old work smarter sort of stuff (line 10). She may also be attempting to preempt a situation whereby she is cast in a negative light by Heke at his planned team meeting by resisting his positioning of her as the kind of manager who would put unreasonable pressure on staff who are claimed to be under stress. 
In both the incidents analysed from this meeting, Jan as the manager was left in an embarrassing situation as a result of what she diplomatically chose to interpret (or perhaps chose to appear to interpret) as genuine misunderstandings. In her candidate explanations for what had happened in each of these instances, she inferred that certain crucial information had not been 'transmitted' or passed properly from one person to another, which was the most neutral possible attribution. The consequences however were, at least potentially, quite serious, in the first case possibly compromising an important collaboration between organisations, and in the second, incurring the displeasure of the CEO and causing a degree of professional and personal embarrassment to Jan herself, and as a result also to Heke. The need to address these immediate problems therefore could not be avoided, thus entailing a different kind of problematic talk. In both cases the issues at stake involved ongoing tasks and relationships, so it was important for Jan to exert her managerial authority in an attempt to prevent a possible recurrence. At the same time, she needed to be mindful of her working relationship with Heke and of his newness in the role of team leader, as well as of her own relationship with the staff and with her superiors.

This helps in part to explain the interactional delicacy with which Jan constructs her interaction with Heke throughout this meeting. In addition, at another level, she is also observably orienting to a number of interactional imperatives arising from the need to balance her at times competing identities and roles as a senior manager, a woman, and a Pakeha within a predominantly male Māori team environment. In this case, the analysis can go no further, as we do not know what transpired after this meeting, but it is quite clear even from the snapshots of data available, that this interaction and its problematic aspects were embedded in a complex fabric of other interactions and relationships, past and future, the negotiation of which took considerable skill and attention from both participants.

\subsubsection{Case 2: 'A subject dear to our hearts'}

The starting point for the second case is a single short interaction, just over six minutes long, between two senior analysts in a government department; the discussion also draws heavily on verbatim transcripts of several sessions run as part of another pilot CED programme. In the focus interaction, Katie and Jo, the two analysts are seated at a table in a shared workroom in the policy unit, and Katie is giving Jo some verbal feedback on the draft of a letter. Katie is providing comments 
and suggestions for improvement. In addition, the two briefly discuss some related issues that arise from the content of the letter. The overall structure and content of this interaction is summarised in Table 5.2 below.

\section{Table 5.2: Case 2 interaction structure}

1 i) Katie identifies first problem (now I just thought this here needed unpacking a bit)

ii) Elaborates and clarifies exact nature of problem with Jo (to start with I wondered whether...)

iii) Proposes solution (in that case put it in a separate sentence...) and provides some further justification to check that her meaning is clear (Katie: see what I mean? Jo: Yeah I do okay)

2 i) Katie identifies next problem ( and then I couldn't understand why you'd said this...); Jo explains (that was moving around the paragraph I think- I'll change it back...)

ii) Katie clarifies (oh I see it's just a bit of a non sequitur).

*DIGRESSION: Joint exploration of the tricky issue being referred to.

iii) Katie offers advice (well I think it would still help to say a little bit about the why)

3 i) Katie identifies next problem (and then the last that I had here...)

ii) Joint clarification of what Jo needs to write (i don't think that they're aiming at the wrong thing)

*DIGRESSION: joint exploration of more general (very complex) issues arising

Katie: $i$ think the effect of Ruth's comment here is that most agencies don't keep the in- (financial) recording information

Jo: $\quad$ no they don't

Katie: a subject dear [laughs]: to our hearts: [laughs] [Digression continues for approximately 1.5 minutes]

iii) They come to a consensus (yeah $i$ agree $i$ don't blame you)

4 i) Jo brings discussion back to what she ought to do, and both discuss how much more time she should spend on this task.

ii) Katie sums up (I think you've already done enough work on it) and Jo provides her intended course of action.

iii) Discussion closes with mutual thanks.

From an analyst's perspective, this interaction seems on the face of it to be completely unproblematic. Although not explicitly stated, the purpose of the interaction is clear, both from what is said and from the contextual information collected at the time which noted that the meeting was prearranged. Katie clearly enacts the role of peer reviewer in a collegial discussion without taking 'ownership' of the task away from Jo. Both state they do not want to spend too much time on it, but the letter raises some issues which are not totally straightforward. These seem to be important and relevant to them both, which appears to justify the more extended 
discussion which takes place at certain points (see $2 *$ and $3 *$ in Table 5.2 below). Despite these digressions, the interaction is clearly structured, all the talk is 'on-task', and a large amount of ground is covered in a relatively short space of time. The tone is friendly and constructive, the interactional style is collaborative, there is a great deal of laughter throughout, and there are no tell-tale signs of interactional discomfort. The interaction appears to result in a satisfactory outcome for both women and for Jo in particular, and this assessment was subsequently endorsed by the participants, who both recalled the interaction even prior to listening to the recording.

This interaction was originally analysed as part of a data sub-set of the LWP baseline corpus to explore the discourse structures and strategies characteristic of informal task-based or problem-solving discussions in backstage workplace settings (Stubbe 1997a, 1998d). In keeping with the principle of appreciative inquiry which looks to identify and build on things that are done well, this particular example was selected as a focus for reflection and discussion with the participants as a mooted example of routine but effective problem-solving talk. Once the participants had been reassured that the 'messy' appearance of the transcripts was actually quite normal for spoken discourse, both the analysts and participants readily concurred that in most ways this was indeed a completely unproblematic example of effective workplace discourse.

The participants initially did feel that the digressions were perhaps somewhat 'inefficient' because strictly speaking they were off-topic, but on reflection they realised that by canvassing issues relevant to other areas of their work, these were really a form of discursive 'multi-tasking' and distributed decision making.

(2.1) Katie reflects on the 'digressions':

... whether we needed to discuss them just to get the letter right or whether we discussed them because they raised items of interest to both of us I wouldn't be quite sure, because clearly the transcript shows that we weren't going to be able to tackle those more complex issues in the scope of the letter anyway, it was beyond what we had the time or the inclination to do ... they certainly have connections, the same kind of issue crops up in other areas ... so in that sense, it's not a digression; but it's probably a bit of a digression away from the very particulars of what was in the actual letter ...

Katie's comment that they raised items of interest in relation to the same kind of issue (that) crops up in other areas also showed a recognition of intertextuality between this particular part of their discussion and others that were ongoing within the team (although this was not the terminology she would have used). 
However, in discussion with the participants, it gradually emerged that while they agreed with the analysts that this interaction would not in itself be considered an example of problematic talk, the analysis did highlight certain typical discursive practices and patterns of communication within the team of which the participants had not been consciously aware previously. In particular, they remarked on the extent to which their talk was heavily embedded in a shared history and set of implicit mutual understandings, and, as exemplified in excerpt (2.2), they thought that this contributed to the effectiveness and efficiency of their communication:

(2.2) Jo on shared understandings in team communication:

\begin{abstract}
... I found reading the written transcript and listening to the tape a totally different experience and I realised that so much of what was going on was non-verbal but a mutual understanding and we were using huge amounts of sort of short hand which I think we do and I was actually sort of thinking in a place like this, where we do have quite a lot of understanding, it's much easier to have efficient conversations than in a place where that understanding isn't taking place; but ... when there are challenges ... you have to take much more time you know, that's a completely different kind of communication ...
\end{abstract}

However, Jo's last comment, when there are challenges ... you have to take much more time, also shows a recognition that implicit assumptions carry a risk of miscommunication. This prompted Jo and Katie to reflect on some recurrent episodes of problematic interaction with members of another unit in the same workplace, and to hypothesise that the patterns we had uncovered in their routine interactions might be implicated as causal factors. In particular, they agreed that discussing matters in a very informal 'by the way' basis, as in the focus interaction, was probably quite a typical pattern within their team, and that this might explain why a consensus often emerged very easily when a topic was discussed in a more structured setting such as a formal meeting. Conversely, as indicated in excerpts (2.3) and (2.4), they thought this could also easily lead to incorrect assumptions being made about the extent of shared knowledge between members of different units, leading to misunderstandings which might easily remain implicit and unresolved.

(2.3) Katie discusses possible sources of inter-unit miscommunication:

... we got some consultants to do a stakeholder survey for us ... ... and most people who wrote into the survey from inside staff moaned like mad about the communications in the place, particularly between units, and it's made me realise that because in the Policy unit, actually probably in all the units, we all rely a huge amount on implicit understanding of all the stuff, that we say things to a member of another unit and they don't necessarily get it, only we don't know they haven't because we've spoken to them as we would to another of our own unit, and they don't understand all the background and can't unpick all the implications without a 
bit of steering of where to go with it; and then we get irritated if they haven't got the point; we get the same kind of coded messages back and we can't work out what the heck they mean because we just don't understand what all the ramifications are; and maybe we all talk in code all the time and nobody can understand each other ...

(2.4) Jo reflects on implicit misunderstandings:

... you end up with that slightly uncomfortable feeling that something's happened and you're not quite sure what it is, and I find it often very difficult to analyse why that's happened, I know it's happened but I don't know why it's happened; it's not always the substance of the words either, sometimes it's just a feeling that the other person isn't on the same wavelength and is giving you messages to say 'I'm not quite sure about this', but using all the right words ...

The discussion then turned to the digression in the focus interaction. Jo commented that this might explain an apparent misunderstanding that had been puzzling her. A similar issue had been the subject of a meeting with another unit in the organisation recently, and some members of that group had complained that the members of Jo's unit had pre-empted the discussion because they had clearly already reached a shared position on it ahead of the inter-team meeting set down to discuss it formally.

Jo reported that she had felt very put out, because she knew her team had not had any such discussions - but she now realised they may actually had done so informally 'in passing' as part of any number of other exchanges where other (related) matters were being discussed, as in the interaction we had been analysing. It was therefore possible that her own team had reached a group consensus, without ever formally setting out to do so, and that this then became the basis of the misunderstanding with the other team. Now they were aware of this dynamic, she felt they would be more able to recognise it and take steps to address it in the future.

This exemplar case has provided a useful illustration of a several analytic issues that can arise in relation to identifying and analysing miscommunication and problematic talk. Firstly, we have seen how difficult it can be in some cases both for analysts and for participants themselves to decide (or agree) whether a particular interaction has problematic elements or not, and/or to get to the bottom of the nature of some kinds of problematic talk when they are noticed. Secondly, lay participants sometimes evaluate aspects of interaction (such as digressions in meetings or overlapping talk) as being problematic, when from the discourse analyst's perspective they are perfectly normal phenomena. Conversely, the outside 'expert' analyst may 'see' an apparent problem where none exists. They may also fail to recognise an instance of 
problematic discourse or a possible trigger for miscommunication because they lack the requisite 'insider' knowledge, or have access to only very partial data.

Determining in any systematic way which aspects of a particular interaction, or indeed the discursive practices of a group or team more generally, might contribute to problematic discourse in other interactions or settings is even more difficult, especially where only brief and mostly unrelated snapshots of data are available, as in the LWP baseline data set. However, this example does demonstrate the potential value to both workplace practitioners and analysts of structured tools for observation and reflection as a means of gaining valuable insights which are likely to be generalisable to other communicative settings. It has also highlighted once again the importance of thick description and of the need to triangulate data sources and interpretations when investigating a complex phenomenon like miscommunication.

\subsubsection{Case 3: 'Power plays'9}

The final exemplar case analysed in this chapter highlights the difficulty of identifying and analysing more global or 'unfocused' types and outcomes of problematic talk at the local level of a single interaction, especially where these have their origins in macro-level ideological or intergroup discourses of power and conflict. In such cases there may be no obvious discourse-internal evidence that there is any problematic element (or at least evidence that is 'objectively' interpretable by an outside analyst). Conversely, the analyst may at times see diagnostic patterns and traces in the discourse which remain invisible to participants themselves or which they may not easily be able to articulate. The discourse analyst must therefore rely on intertextual analysis, ethnographic fieldwork, and engaging research participants in a reflexive process to be able to usefully interpret such interactions from the perspective of the participants in a particular workplace context. By the same token, workplace practitioners cannot necessarily rely on 'outside experts' to 'diagnose' such issues or offer workable solutions without this kind of situated understanding.

This example also illustrates the complex interplay between different levels and dimensions of analysis. As in this instance, a given interaction might be analysed as being perfectly functional and effective at one level, such as conveying information

\footnotetext{
${ }^{9}$ Related analyses of this set of interactions have appeared in previous publications (Stubbe 1998b; Stubbe and Holmes 2000; Holmes and Stubbe 2003a).
} 
and/or achieving a set of task-related goals, while at the same time it may be defined as problematic at the relational and/or affective levels. To complicate matters, different participants may also have different perspectives and levels of awareness, and this will also affect the degree to which any such problems can be mitigated or repaired.

The interaction in question constitutes a one hour meeting between Aidan, who is Māori, and Hugh, a Pakeha colleague. The two men are evaluating vocational training programmes for Māori students. Aidan and Hugh are peers within the organisation, and as illustrated in example (3.1), a typical excerpt from this interaction, there is little overt evidence from the discourse itself that there is anything particularly problematic going on here.

(3.1)

Context: Advisors in a government organisation evaluating proposals.

Hugh: $\quad$ yeah um the trainees finding their own so there could ++ and that's a flaw with trainees finding their own is that they could end up sweeping the floor for two weeks

Aidan: yeah

Hugh: um + and by having the trainee find it the polytechnic gets no input into the the training that's covered

Aidan: yeah well the other issue about that thing is that the they're they're not they're not seeking culturally safe industries places to $/ /$ protect $\backslash$ students

Hugh: /yeah $\$

Aidan: and so all that sort of stuff

Hugh: yeah yep

Aidan: yep um

Hugh: well like there are other ways of making it culturally safe

Aidan: yeah

Hugh: I mean I I I I mean it's nice to have cultural safety but I think part of that is is the realisation that it's not a culturally safe environment + out in industry

Aidan: no and yet in the cultural component they're not teaching any of that stuff about how to deal with that

Hugh: right okay yep + yep

Aidan: and um yeah so the other thing is so how are they supporting students + into into industry you know those sorts of things

Hugh: yeah

Whilst this is a very task-focused interaction, at a superficial level at least, it is consistently polite and friendly. Overall, the two men appear to be engaged in a relatively smooth and uncontroversial discussion where they meet their stated goals in terms of completing a joint task. There is little or no direct manifestation of conflict, problematic talk or miscommunication in the discourse. Nevertheless, the ethnographic data, together with comparative data from other interactions involving 
Aidan in particular, provided evidence of undetected problems which formed an undercurrent to this interaction.

An interview with Aidan provided the first real indication that he had perceived this interaction with Hugh to have been problematic, and that this was part of an historical situation. (We do not have any comparable information on how Hugh perceived things, so this is necessarily a partial view.) Interestingly, it was not the practical outcome of the interaction that Aidan saw as the problem - he considered that they had completed the task expeditiously and without major disagreement. Rather, as indicated in excerpt (3.2) from the ethnographic interview, the issue was that Aidan felt uncomfortable with Hugh's style of interaction, and considered there to be unresolved conflict between them, which essentially boiled down to a kind of power struggle at both an interpersonal and intergroup level.

\title{
(3.2) Interview excerpt:
}

\begin{abstract}
now with Hugh, there's a history between me and Hugh, and he's a Pakeha male who brings all those power elements of Māori/Pakeha into what I believe is a Māori process ... and Hugh I believe was forever arguing. ... I think what I'm doing here is saying, to hell with all that bullshit, I just want to get the job done, and I'm not going to playing your game of arguing ... he's easy to get on with, this guy, he's not ugly or anything, I'm just really conscious that he power plays
\end{abstract}

Aidan reported that he resented and resisted these "power plays" by, maintaining a degree of distance between himself and Hugh, refusing for instance to be drawn into extended discussion of a topic, and instead just focusing on the task at hand.

\section{(3.3) Interview excerpt:}

I got to the point of saying to this man, you've got a lot to say but there's actually very little substance to what you're saying. I don't know if that's a Pakeha trick in terms of communication, but whenever things get tough it seems to be you need a thousand words to explain what you could explain in five and to me it seems to be a disempowering language technique that if you possibly come up against an articulate minority person, then the way to get around that is to bamboozle with words and jargon; and this guy was really good at that, and I got to the point of saying, I'm not dealing with any of that, we've got a job to do.

The covert problem is thus a problem involving both interpersonal and societal power issues, although it is never articulated overtly between the men. Rather it is played out in the way the discourse is distributed and instantiated between the participants. While the interaction between Aidan and Hugh we saw in (3.1) above is at the informal end of the scale, it is very on-task and focused, and there are few explicit signals of solidarity or high involvement evident. Instead, Aidan makes 
assertive use of "one-at-a-time" turn-taking strategies (Coates 2004), and consistently addresses direct questions and challenges to Hugh. In these ways, Aidan manages to present himself both as an able and confident professional in his field who knows what he is talking about, especially in terms of Māori issues, and to consciously place himself on an equal footing with Hugh. In the data available, Aidan also avoids as far as possible going on record with any disagreements, and, as we see in excerpt (3.4), when he does, he tends to do so fairly minimally and with a degree of mitigation. Thus in line 3 he softens his challenge by formulating it indirectly and using Hugh's name (that's words though Hugh), while in line 6, he uses a similar indirect disagreement, echoing the word though and again softening the threat to face by means of the addressee-oriented endtag eh: (not everybody does though eh).

\section{(3.4)}

1 Hugh: I guess to make me um perfectly happy with that sort of thing

2 I'd like to say the following w- we tutors use

3 Aidan: that's words though Hugh

4 Hugh: I I know and and perhaps concreting that + I mean so

5 I mean every nobody could rattle off that list the crucial

6 Aidan: not everybody does though eh

7 Hugh: yeah but and anyone could put it in there but do they do it +

8 and to make me perfectly happy

9 I'd like to see examples of um group learning

10 or a description of I'm nitpicking

11 Aidan: $\mathrm{mm}$

12 Hugh: but (I mean) in terms of an ideal + yeah

13 I'd like to see how that that integrates into the whole teaching package

14 think this isn't so bad but a lot of the proposals we're looking at

15 they- they kind of skimp over it

16 and they might give it a couple of mentions

17 like that list or something (though) you're not you haven't got a picture of

18 + you know what (it takes)

19 Aidan: is that a gap then

20 is that what something you want you'd want me to write down

This example illustrates Aidan's stated strategy for dealing with Hugh: namely, he tends not to elaborate or get drawn into an extended discussion. Rather he lets Hugh talk with only the most minimal of feedback, and then changes the subject: is that a gap then (line 19). This appears to be a polite strategy for avoiding conflict, but it is one which is so subtle that it only becomes apparent after being alerted to it via a 
follow-up interview (as noted in interview excerpts (3.2) and (3.3) above) and by comparing it with Aidan's usual style of interaction with other colleagues.

A comparison of this interaction with another parallel discussion between Aidan and Vince, a Māori colleague which relates to the same evaluation task provides some interesting insights into the discourse strategies Aidan used to avoid overt conflict and mask his feelings about Hugh's style of interaction, while at the same time indirectly resisting what he sees as Hugh's 'power games'. There is no overt status difference between any of the interactants, who are all of similar age and educational background, and work at the same level in the organisation. ${ }^{10}$ Nevertheless, despite the contextual similarities, there is a marked contrast between Aidan's conversational style when he interacts with Hugh and when he is talking with Vince. As we see in the next excerpt, Aidan and Vince, who know each other very well and interact regularly outside the work context, appear to place a high value on creating and maintaining solidarity through their interactional style.

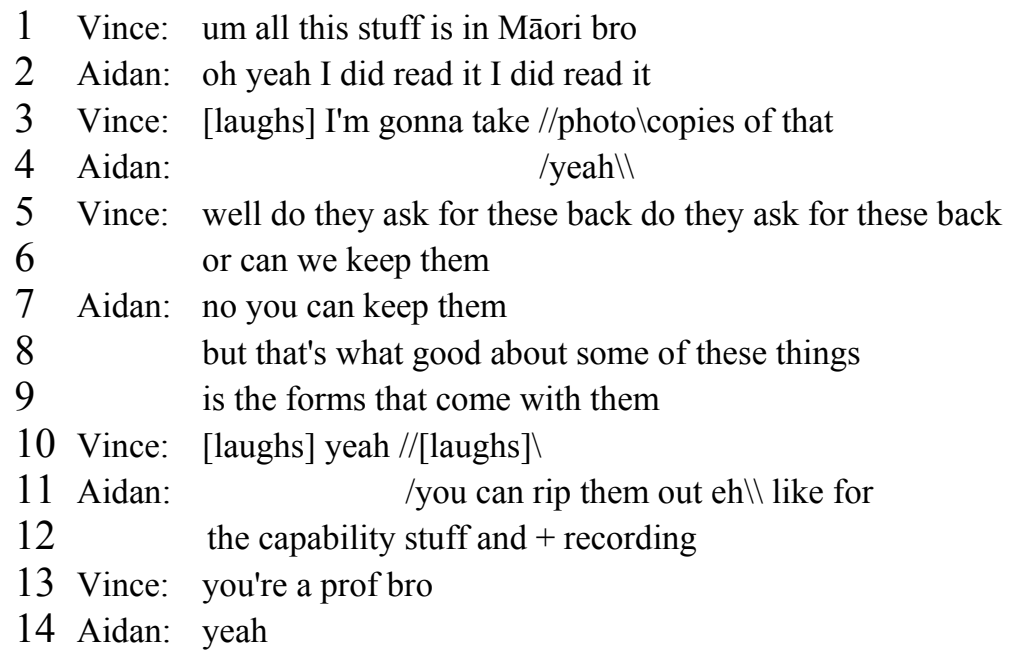

Although the two men are obviously involved in a serious task, they adopt a very informal style, and make active use of a wide range of positive politeness strategies designed to maximise the level of solidarity between them, and to reinforce the construction of this as an interaction between Māori men who are both friends and colleagues in a predominantly Pakeha organisation. For example, they frequently

\footnotetext{
${ }^{10}$ This does not mean there are no power differences. Aidan clearly has 'expert power' by contrast with Vince, who is relatively new to the job, and both interactions take place in a context where unequal power relations between Māori and Pakeha in New Zealand society are foregrounded.
} 
address one another as bro (lines 1,13), there is a lot of humour and laughter, and there are frequent brief digressions into relevant but strictly speaking "off-topic" talk. As reported in his interviews, Aidan is also motivated by a wish to help Vince, who is a friend as well as a Māori colleague, "get up to speed" with his new job. The discussion of each point therefore often takes longer than if they were focusing purely on the task at hand, as Aidan takes the time to explain things or provide advice. Aidan's wielding of "expert power" is humorously acknowledged by Vince in the excerpt above when he quips you're a prof bro (line 13), but in fact, Aidan is careful not to dominate the discussion. Rather, his disagreements are heavily mitigated, as we see in excerpt (3.6), where Aidan takes issue with Vince's criticism of a proposal for over-emphasising academic content (something which he does regularly and vigorously throughout this interaction).

\section{(3.6)}

1 Aidan: the only thing that I would probably contest you about

2 the um about the university approach

3 and that I think cos it's a bridging course to university

4 these guys hopefully gonna feed onto there

5 Vince: $\mathrm{mm}$

6 Aidan: so I think that they actually do need an introduction

7 to that type of um approach

8 but I think that the crux of it is is

9 that they're encased in [in māori] tikanga maaori

10 because they're outside the university

11 Vince: yeah well after writing that I reflected on that and thought

12 yeah no I'm just being I'm just reacting [tut] to um academic snobbery

13 Aidan: shall I take that off

14 Vince: yeah take it off

15 Aidan: apart from that I've just got what you've got just in a lot less words

$16 \quad / /[$ laughs $] \backslash$

17 Vince: /[laughs] you have to draw it up though $\backslash \backslash$

18 Aidan: [laughs] that's a different approach you summarise things

19 and put down your summary thoughts

20 whereas I look for evidence and and bang it out bit by bit +

21 I think that's what I'm doing anyway [tut]

22 where you're very good at summarising things and putting it down

Aidan's mitigation takes the form of strategies such as minimising e.g.the only thing and hedging e.g. I would probably contest you (line 1), I think (lines 3, 6, 8, 21), actually (line 6), just (line 15), elaboration and providing reasons (lines 1-7), and positive politeness strategies such as giving praise (lines 15-22). This is typical of Aidan's interactional style with Vince. 
In summary, in his interaction with Hugh, Aidan uses a combination of positive and negative politeness strategies to keep the interaction flowing as smoothly as required in order to complete the task, but without investing any great effort into the solidarity aspects of the longer term relationship as he seems to do with Vince. The effort that is required to maintain a degree of social distance and resist Hugh's enactment of power, while at the same time keeping up a show of friendliness and collaboration, is probably one source of Aidan's perception of this as an instance of problematic talk. In addition, the history of his relationship and previous interactions with Hugh undoubtedly also contribute to this perception. By comparing the strategies Aidan uses in these two comparable but differently constructed interactions, we gain some insight into how people can make use of quite subtle differences in discourse strategies and styles to constructively negotiate problematic issues such as power, conflict and disagreement in otherwise collegial workplace relationships.

\subsection{Refining the analytic approach}

The heuristic framework presented in section 5.2 above aims to provide an analytic toolkit for analysing real world cases of miscommunication and problematic talk that is sufficiently 'spacious' to permit the flexible and integrated application of different perspectives, analytic dimensions and methods of analysis to an inductive thick description of whatever data is available. In real life, unlike in academia, people do not often have the luxury of looking in depth at just one little 'slice' of a complex issue or episode or tracing it back in any detail. Instead, they want to be able to 'solve the problem' and work out 'what's happened/happening here?' in a holistic way, often 'on line' and in real time. This framework was therefore deliberately designed so that the analytic process would closely parallel the naturalistic action-reflection processes adopted in the communication evaluation development (CED) model used with the workplace research participants, and indeed grew out of that participatory process in many respects. The aim was to provide an empirically robust platform for an integrated analysis which would also have practical relevance from a workplace practitioner perspective.

As we have seen in the illustrative examples from the baseline data in this chapter, any attempt to analyse miscommunication and problematic talk systematically in 
natural workplace settings has to account for the complexity and interdiscursivity of the context as well as of the phenomena themselves. Spoken interactions take place within a rich discursive fabric where communication, relationships, and practical work-related consequences are already tightly interwoven. In the case of miscommunicative or problematic interactions, identifying the actual (often underlying) matter at stake often involves unravelling the threads in order to trace an issue back to precursors or triggering incidents, analysing other contributing factors and taking a range of perspectives and possibilities into account. For the participants themselves, it may also mean engaging in various forms of 'damage control' in order to contain, repair, mitigate or resolve the downstream effects or consequences of some piece of problematic talk, whether these be actual or potential.

As a working model to guide the analysis of the baseline examples, the integrated analytic framework presented here has demonstrated a number of strengths. It provides the analyst with a set of heuristics against which to test initial identifications, systematically create descriptions and develop alternative readings of the data. It also facilitates the teasing out of different layers and dimensions of observed or reported episodes of miscommunication and problematic talk, and of the strategies used by workplace participants to manage these. By allowing multiple 'entry points' into the analysis of a candidate episode, it has also been possible within this framework to accommodate, even take advantage of, the intertextuality and context-embeddedness of workplace communication which is the hallmark of any dynamic complex system, thus greatly enriching the analysis. Taking a flexible, pluralistic and multi-layered approach like this has also added value to existing unidimensional tools and approaches by making it possible to identify a number of complexities in the cases analysed which may otherwise have remained occluded.

Naturally, there are also limitations to be noted. Firstly, this working model does not attempt to provide an integrated theory or a unified methodological approach, although, as already discussed in chapter 3, it remains open to question whether this is an achievable goal, or even a desirable one. Rather I have sought to provide a logical framework to accommodate a range of existing perspectives and tools in a working model for analysing miscommunication and problematic talk so that these can be applied in a rigorous and useful way in workplace settings. 
Secondly, for this approach to work to best advantage, it requires a particular kind of research design and data collection methodology. There needs to be a mix of ethnographic and interactional data which allows for the tracking of intertextual and inter-contextual links, and ideally some kind of participatory or action research component to facilitate a close engagement between researchers and workplace participants. One important limitation of the baseline data set examined for the purposes of developing and evaluating the working model is that the original LWP methodology was not designed for the purposive collection of related interactions in quite this way. As we have seen in the exemplar cases in this chapter, it was insights gained from those sets of related interactions that were sometimes collected by chance, along with input from the pilot action research data, which showed the importance of examining intertextual links using an integrated approach. But because of the way in which it was collected, what the baseline data set has not allowed is a systematic combination of comprehensive ethnographic data with detailed discourse and thematic analysis of sets of related interactions. This limits the degree to which it is possible to analyse miscommunication and problematic talk as part of a complex workplace system, something which the analysis of the examples in this chapter has suggested as a necessary next step.

As a way of assessing the value and feasibility of taking such an approach, the next two chapters present a detailed longitudinal case study of the communication patterns and issues observed in data collected from a factory production team in a subsequent phase of the research, as described in chapter 4 . These chapters also examine the strategies employed by the team members and their team coordinator to 'manage' miscommunication and problematic talk in this challenging environment. Chapter 6 evaluates the team's communication problems and strategies within a community of practice framework as a way of enhancing the contextual dimension of the analytic framework already presented. Chapter 7 builds on this further by intensively tracking the interactions of the team coordinator and exploring the ways in which she manages the contradictions inherent in her role, thus adding a critical perspective to the analysis. 


\title{
6 (Not)' ${ }^{6}$ getting the message across'? Problematic talk on the factory floor
}

\author{
Manufacturers and packers talk over the intercom: \\ David: $b d$ three ninety nine my nigger \\ Peter: thank you brother you are the best bro ... \\ David: sweet as bro sweet as \\ Tony: copy peter is the [product] coming down \\ Peter: yeah bro one point three there \\ David: did you ring up Ginette and tell her
}

\subsection{Introduction}

This chapter presents the first section of an in-depth case study of the discursive practices used by members of a factory production team as they exchange information, solve problems and attempt to persuade others to do things during the course of routine shifts. The focus is on the communication difficulties faced by these workers in their interactions with one another on the factory floor, and on the strategies they can be observed to use to optimise their communication in this challenging environment. As previously outlined in chapter 4, the approach taken to this complex case study is an in-depth situated analysis or thick description of a multiplex set of interactional and ethnographic data gathered intensively over several multi-day shift periods. This further extends the conceptual analysis of miscommunication and problematic talk presented in chapter 5 by showing how a case study approach can account for the complexities of problematic workplace discourse more comprehensively than is possible via analysis of a largely decontextualised collection of single examples.

As illustrated in the opening excerpt and again below, it would be very difficult in a context-bound environment such as that of the factory studied here for an analyst to unpack the layers of meaning and interpret the significance of much of the talk without recourse to the kinds of detailed ethnographic techniques advocated by 
interactional sociolinguists (e.g. Gumperz 1999; Roberts, Davies and Jupp 1992; Eckert and McConnell-Ginet 1992, 2003). As discussed in chapter 4, the analytic tools used for this case study thus fit broadly within a critical interactional sociolinguistics. In addition, as will be demonstrated below, the unique workplace culture of the team studied here also needs to be taken into account quite explicitly, over and above the more straightforward contextual factors usually considered in sociolinguistic analyses such as the physical setting and the background knowledge and technical jargon which participants bring to each interaction. The general patterns of communication observed, and the particular ways in which selected instances of miscommunication and problematic talk unfold and are addressed within this team, are therefore framed here within a discursive community of practice model.

The case study methodology and reporting of the findings in this chapter are organised into two parts, roughly following the analytic sequence laid out in chapter 4 (see 4.2.2). The next section introduces the factory team which is the focus of the case study and provides an ethnographic overview of its working environment, including the communicative challenges faced by team members. This information is based on the ethnographic data obtained in the course of collecting the recorded data and the subsequent action research process, together with a thematic analysis of the full interactional corpus. Section 6.3 combines this ethnographic data with a close analysis of selected interactional sequences to describe the discursive patterns which characterise this team as a community of practice which, along with more specific strategies identified as contributing to the accurate and efficient exchange of vital information and troubleshooting of problems, function as important resources for preventing and/or repairing miscommunication.

\subsection{Ethnographic overview: the Power Rangers ${ }^{1}$}

\subsubsection{The team and its working environment}

As detailed in chapter 4, this case study was initiated as part of a collaborative action research project involving university researchers and production staff at a large plant

\footnotetext{
${ }^{1}$ The name of this team, the 'Power Rangers', is a pseudonym, as are all other names included in the data excerpts and associated discussion.
} 
which manufactures soap and personal hygiene products for the New Zealand market. At the time the data was collected, the Power Rangers team was one of four self-managing teams in the 'powders' department of this factory which were rostered to cover a 24 hour/seven days-a-week operation. ${ }^{2}$ In line with the management's philosophy of promoting self-managing teams, all production teams in the factory had selected a name by which they were known throughout the plant, usually something humourous or tongue-in-cheek inspired by the popular media (cartoons, films) or well-known sports teams.

The Power Rangers were identified by the factory management as their topperforming production team on the basis of an excellent record in meeting production and quality targets, their safety record (i.e. no 'lost days' due to accidents), and the fact that, rather unusually within such a large factory, this team had generated no industrial or personal grievances for some considerable period of time. The management were keen to understand the positive dynamics of this team and its communication processes, with a view to coaching other teams at the factory to emulate those aspects which led to their success, whilst the team itself and the team coordinator (TCO) were interested in identifying ways to further improve their own performance. At the same time, aspects of the Power Rangers' unique communicative culture were somewhat at odds with certain company policies and expectations of interpersonal conduct (see 6.3 below), something to which team members were clearly also very sensitive. ${ }^{3}$ Whilst the managers were prepared to exercise a degree of tolerance in the case of this team because it had clearly developed a 'winning formula' (and the team seldom went 'too far'), they did not wish to encourage other teams to emulate what they saw as less desirable behaviours. As described in chapter 4, at a practical level, the Power Rangers team worked in two separate, vertically adjoining areas. One was a manufacturing space upstairs, where operations were monitored both directly and from a computerised control room by technically skilled staff who co-ordinated the production of various soap powder

${ }^{2}$ Two day shift and two night shift teams covered this round the clock operation between them, each working four 12-hour days - four days on and four days off.

3 This was an important issue when it came to engaging the team in the study and establishing mutual trust. For instance, members wanted reassurance that they would be able to 'vet' any findings before they were reported back and that their recordings would not be made available to management, who they feared might disapprove of their selfacknowledged 'over the top' style of interaction. 
products. The manufacturing sub-team could also call, as required, on in-house engineers and chemists, who were a factory-wide 'shared resource', not members of this team, and certain members of the packing team were also occasionally seconded to this area. The second workspace was the packing line on the factory's ground floor immediately below, which was connected to the manufacturing area by a metal staircase and by a series of large chutes and hoppers. The packing line workers moved around a lot as they actively monitored a series of automated production lines which packed soap powder into boxes as it came down the chutes. This was a very noisy and bustling hard hat environment, with forklifts moving in and out of marked 'roadways' constantly to stack and move boxes and pallets. Regular liaison had to be maintained between the manufacturers and the packing line, mainly through the use of the factory-wide intercom radio system or via telephone calls, along with occasional physical visits back and forth. The two groups had separate daily briefing meetings, with the TCO acting as go-between. The packing team met together as a whole group once a day at the start of each 12-hour shift. The manufacturers held their own short briefing separately, but being a smaller group with their own base and tearoom, tended to have more informal contact throughout the day as a group. The packing staff shared a larger 'smoko room"4 with members of other downstairs teams and had strictly rostered break times as the lines had to remain staffed at all times.

At the time of data collection, this close-knit team had 22 core members, 18 of whom were male. Four different ethnic groups were represented; in order of respective size these were Samoan, Tongan, Māori, and Pakeha (i.e. European New Zealanders). Over half the team were of Pacific Island ethnicity, and the majority of these workers spoke English as a second language. Team members had a wide range of educational and vocational backgrounds, some with full trades qualifications (e.g. fitters and technicians) while a number had no formal qualifications at all. Many team members had worked together for a very long time and had developed a strong sense of group identity. They were highly motivated to out-perform other teams within the factory, and to meet or exceed production targets and quality and safety requirements. As a result, staff turnover in this particular team was low, and members of other teams at

\footnotetext{
4 'Smoko' is an idiomatic expression for a workplace tea or lunch break in New Zealand English.
} 
the factory regularly requested transfers to the Power Rangers which was seen as a desirable team in which to work.

The TCO was a Samoan woman in her mid 30s; she was equally fluent in both English and Samoan, and was extremely well-respected and highly thought of by her own team, other workers and TCO's in the factory, and factory management alike. The official ethos in the factory was that all teams were considered to be 'selfmanaging', with the team coordinator role seen, on paper at least, as 'first amongst equals'. In practice, however, the TCO in this team, as in others, functioned more as a middle manager. This particular team leader had been identified as a star performer by the factory management; she was therefore regularly seconded to special projects elsewhere in the factory, and also contributed to a comprehensive raft of in-house training, mentoring and quality improvement programmes. Several other team members had also been trained and mentored by her to allow them deputise as 'acting TCO' when required, and/or to take on leading hand roles in specified areas of work.

\subsubsection{Communication challenges}

The action research plan agreed with the Power Rangers team and the factory management specified that the research team would provide and receive feedback on analytic work in progress throughout the data collection phase, and would then report back the results of an initial analysis of the full data set within 6 months after completion of the data collection. ${ }^{5}$ The first stage of the data analysis therefore comprised a descriptive survey of the patterns observed in the team's everyday communication as a basis for evaluating the team's strengths and areas for development, within an appreciative inquiry framework (see chapter 4). This overview analysis was based on ethnographic data from participant observation, interviews with key informants, feedback sessions and document analysis, together with the results of a thematic content analysis of recurring themes in the full corpus of interactional data. The initial scanning of the data set highlighted some general communicative challenges faced by team members in this environment, as well as

\footnotetext{
${ }^{5}$ A multimedia training resource kit on effective communication (Talk that Works) was produced subsequently. This was based mainly on the initial ethnographic analysis in addition to material drawn from the micro-analysis of interactional data discussed later in this chapter and the next, in conjunction with video recorded interactions and interviews from another factory (Stubbe and Brown 2002).
} 
more specific issues arising from recurring themes within the set of interactions recorded during each shift. It also identified a number of the discourse practices and strategies that might be helping this team to manage such issues effectively, thus providing a starting point for subsequent more in-depth analysis and longitudinal tracking of the interactional data, as reported in section 6.3 below.

An outsider observing the Power Rangers at work on the factory floor could not fail to be struck by the range and complexity of the communicative demands placed on the workers in this environment. Taken collectively, these clearly had the potential to become real barriers to successful communication in some cases. Firstly, there were the obvious physical aspects of working in a factory environment, such as the constant noise and the separation of team members into different locations which reduced both the ease and frequency of face-to-face communication. Interactions on the factory floor were often rather sporadic and predominantly involved the routine imparting of specific information or instructions, along with a certain amount of social talk and banter, punctuated by episodes of troubleshooting or problem-solving talk. The factory staff's own perception was that team members, especially those working on the packing lines, did not really talk to one another very much. In fact, the recordings showed that, despite its intermittent nature, a surprising amount of face-to-face verbal interaction did take place in this context, supported by other modes of communication such as signs and gestures, and talking over the factory intercom system.

Secondly, there was tremendous variation in the complexity of the communicative tasks which may be required during the course of a shift. These demands varied from individual to individual according to their particular roles, but ranged between very brief and intermittent routine interactions breaking up long periods of solitary work, to quite involved troubleshooting and problem-solving discussions (often conducted under pressure), through to participating in on-site training or representing the team at factory-level meetings dealing with complex issues such as quality assurance. It was also clear that recent changes to production procedures and the recent move towards self-managing teams had placed greater communicative demands on both individuals and teams in terms of their English language proficiency and literacy, and also their interpersonal and intergroup communication skills. For example, expectations of cooperative teamwork were increasingly replacing the previous more 
hierarchical and directive management structures, and changes to quality assurance processes had also led to an increase in the amount and complexity of documentation. This latter point was particularly problematic for those workers who had been at the factory for a number of years, and who had not previously needed this level of communicative competency to undertake skilled and responsible tasks ${ }^{6}$.

Finally, the factory workforce as a whole was very diverse in terms of sociodemographic factors such as gender, age, cultural and linguistic background, as well as workers' technical and/or educational backgrounds, their practical experience, and their literacy and numeracy skills. This diversity placed great demands on team members' interpersonal skills in particular, as well as making communication at a purely transactional level more problematic than it would be in a less heterogeneous environment. Related literacy issues meant that spoken interaction was the primary channel of communication for many workers on the factory floor, although written and electronic documentation of various kinds was by this time becoming an increasingly essential component of the production process here as in other factories (Roberts 1999; Waldvogel 2005). In addition, although the official language of communication in the factory was English, the workforce was multicultural and included many people for whom English was a second language. In the Power Rangers team, a critical mass of Samoan and Tongan speakers meant it was not uncommon for code switching between English and these other languages to occur in both task-related and social contexts (see Holmes and Stubbe 2004), and, somewhat unusually for New Zealand factories at this time, this practice was not actively discouraged, although it did occasionally create discord between team members from different ethnic groups.

\subsubsection{Sources of miscommunication}

The Power Rangers team worked in a challenging physical, technical, and social environment where they were required to meet consistently high standards of safety, quality and productivity, while at the same time maintaining good working relationships with a diverse set of co-workers. Predictably, tensions regularly arose between competing demands in the course of their daily work, and there were

\footnotetext{
${ }^{6}$ This was pointed out to us by the TCO, and was also raised during some exploratory interviews conducted at the factory later in the same year (Roberts 1999) as part of a separate study of the needs of English as a Second Language (ESOL) workers in NZ workplaces.
} 
numerous practical constraints and predisposing factors for problems to occur which were often very complex to resolve. Completion of routine tasks and practical problem-solving often required a great deal of negotiation and coordination between multiple parties with different levels of expertise and skill sets, as well as a need to remember who needed to be informed (and when) of any problems arising, and of the outcomes or required follow-up actions.

The analysis of recurring themes in the interactional data set summarised in Table 6.1 below provides a clear demonstration of the extent to which miscommunication and problematic discourse were ongoing occupational hazards in this complex environment. The potential for miscommunication to occur at a number of levels was therefore great, and as detailed, such miscommunication could be highly consequential in any of a number of ways. Where the effectiveness and accuracy of information exchange and understanding were affected, the practical consequences were often obvious and immediate. At the level of interpersonal and intergroup relationships, miscommunicative discourse could result in longer-term, less visible damage to motivation, morale and team spirit, which in turn might affect productivity and safety.

However, the initial survey of the data and subsequent more in-depth analyses confirmed the 'bottom line' evaluation by the factory management that, on the whole, beneath its rough and ready surface manifestations, communication within the Power Rangers team worked very smoothly and effectively. As will be further elaborated in chapter 7, detailed tracking of the TCO's discursive practices through a series of interactions also revealed that she had a sophisticated understanding of this reality, and of the importance of managing these risks in a flexible and proactive manner. Despite the sometimes volatile mix of individuals involved, and the huge potential for communicative difficulties outlined above, there were thus surprisingly few clear examples of ineffective communication as defined in terms of negative outcomes at a practical or interpersonal level such as, for instance, significant production problems or tension between members of the team.

As in any workplace, it was, of course, possible to identify numerous examples of localised miscommunication or problematic talk which required a degree of effort on the part of the participants to work through or repair. However, from a purely practical perspective, within the Power Rangers team these instances of actual or 
potential miscommunication rarely seemed to lead to major problems and issues seldom remained unresolved for any length of time.

Table 6.1: Recurring issues

Issue
Technical problems
Examples: packaging and glue problems on the
packing line; issues with powder quality; minor
equipment malfunctions or misalignments.
Logistical problems
Examples: faulty estimates of size and/or timing
of powder production runs; under-ordering of
materials required (e.g. quantity of cartons,
glue); poor coordination of production and
routine activities (e.g. cleaning, machinery
maintenance).

\section{Major faults or breakdowns}

Examples: frequent jamming of the 'elevator', a machine which automates the stacking of cases onto pallets from the conveyor belts on the packing line; problems with the 'checkweigher' which ejected packets outside the permissible weight range from the conveyor belt.

\section{Failure to follow correct procedures}

Examples: non-implementation of required changes to documentation (e.g. entering packing codes, filling out log sheets); recording errors (e.g. incorrect weights on product cartons); incorrect stacking or wrapping of boxes; unsafe operation of equipment.

\section{Intergroup miscommunication or conflict}

Examples: inadequate liaison or lack of cooperation on tasks or problem solving between sub-groups within the team (such as the manufacturers, packers and fitters) or misunderstandings between team members and other factory staff or departments (such as stores, quality assurance, office staff).

\section{Staff management issues}

Examples: unexplained absences, lateness, noncompliance with rostered break times, shirking of chores such as sweeping up, working excessive overtime, rotation of duties, contested eligibility for training or increased responsibilities.

\section{Consequences}

Team members/production supervisors had to maintain constant vigilance; problems here resulted in rejection and requirement to 'rework' large quantities of product already packaged.

Lack of timely effective communication resulted in a miscalculation of how many tonnes of a particular powder were required to complete a production run, a costly mis-timing of the point at which hoppers and chutes need to be cleaned out in readiness for a new product, and shortfalls in materials at a crucial point in the process.

These problems affected the quality and/or efficiency of production, because they often resulted in stoppages and/or staff having to switch to manual operations such as hand stacking onto pallets. The resulting pressure to solve problems quickly often led to frustration, miscommunication and, occasionally, disputes.

These frequently recurring quality and safety issues affected process and quality control, and could result in censure by and/or poor relations with other departments, a need to redo work and consequent reductions in productivity, loss of face for the individual involved and/or for the team as a whole.

Such issues could be one-off or ongoing, and had both practical and interpersonal consequences which affected productivity and team morale. They often related to a lack of clarity (on either side) about expectations and procedures, or perceptions of tardy action which could rebound on the productivity and reputation of individuals, groups or the team.

As a self-managing team, any member could legitimately raise such issues with colleagues, offer advice or make a complaint, and this often led to interpersonal misunderstandings and conflict which could escalate if not promptly resolved. 
It was also clear from the analysis that team members had a variety of effective communicative processes and strategies at their disposal for dealing with issues that arose and/or for preventing problems from arising in the first place, which contributed in no small measure to their performance as a well-motivated and highperforming production team. These strategies operated at different linguistic and discursive levels, and could be seen to address a number of the instrumental, interpersonal and intergroup dimensions of communication identified as relevant in the analysis of miscommunication and problematic talk in the previous chapter.

However, what also became very apparent as a result of the next stage of analysis was that neither problematic sequences of talk nor the discourse strategies used to manage these instances can be properly understood in isolation. On the basis of the descriptive analysis above, the Power Rangers' working environment can reasonably be characterised as comprising a number of overlapping open-ended activity systems and genre networks, and it could therefore be argued to fit the definition of a complex adaptive system (as discussed in chapters 2 and 3). The next section provides an analysis of the strategies typically used by the members of Power Rangers team to optimise their communication within this complex system, and shows how framing this team as a distinctive community of practice helps account for the discursive patterns observed.

\subsection{The Power Rangers team: a discursive community of practice}

A community of practice approach to studying workplace communication and miscommunication recognises that workgroups evolve their own unique locally situated communicative practices, linguistic repertoires and shared understandings over time, through a mutual engagement in tasks and their co-membership of the group (Wenger 1998; Holmes and Meyerhoff 1999; Eckert and McConnell-Ginet 1992, 2003; see also chapter 2 - 2.3.3 and 2.3.6). In the words of Eckert and McConnell-Ginet, a community of practice is "an aggregate of people who come together around mutual engagement in an endeavour. Ways of doing things, ways of talking, beliefs, values, power relations - in short, practices - emerge in the course of this mutual endeavour." (1992:464). The community of practice approach focuses on 
what members do - the practices or activities which indicate that they belong to the group and the extent to which they are core or more marginal members.

Wenger (1998:73) identifies three criterial features of a community of practice: (i) mutual engagement; (ii) a joint negotiated enterprise; and (iii) a shared repertoire of negotiable resources accumulated over time. Shared discursive repertoires and repeated patterns of interaction can both be seen as "constitutive characteristics" of a community of practice (Wenger 1998:125-126), and hence as means by which a distinctive workplace culture can be constructed. At another level of analysis, patterns of participation in communities of practice also provide "an important link between each individual's experience and the larger social order" (Eckert and McConnell-Ginet 2003:57). In this way a community of practice framework can take account of the attitudes, beliefs, values and social relations which underlie situated, group-specific practices, and, as will be shown in chapter 7, can also accommodate the application of a critical as well as a descriptive lens.

On the basis of Wenger's three criterial features, the Power Rangers team constituted a very tight-knit and highly cohesive community of practice at the time of their participation in the study. With regard to the first criterion, talk was regarded as a means to a practical end, rather than as the main currency of work as in the government office workplaces described in chapter 5, and the apparent level of mutual engagement between team members on a day-to-day basis was not uniformly high. Nevertheless, this team was quite clearly a very cohesive group which enjoyed sustained and multiplex mutual relationships. As we saw earlier, they had daily briefing sessions, individuals regularly engaged with one another in the course of their 12-hour shifts on the shopfloor and in their breaks using a variety of communication channels, and many team members also had regular social contact outside work hours. The Power Rangers team also had a strong sense of group identity and a real sense of joint enterprise, focused on completing the immediate tasks during a shift, as well as meeting longer term goals such as continuing to outperform other production teams. Teamwork was highly and explicitly valued, an ethos which was further reinforced by the traditional privileging of the group over individuals in the Polynesian cultural background of a majority of the team.

One of the more noticeable ways in which the three criterial characteristics were reflected in the team's discourse was in a strong orientation to team morale, and a 
very distinctive sparky, contestive and humorous communicative style. The team used many markers of solidarity in their interactions, with a high proportion of jocular abuse and other forms of humour, in-group talk and gossip. The Power Rangers had a well-deserved reputation at the factory for uninhibited swearing, and constantly, as they put it themselves, 'joking around', 'playing silly buggers', and 'having each other on' which sat alongside their status as the top-performing team in the factory. At the time of the study, their particular blend of verbal humour, jocular abuse and practical jokes contributed to a team culture and ethos which was unique within the wider factory community, and which helped to create generally very positive relationships within the team along with a strong sense of team identity.

The interactions recorded by this team were also highly context-embedded, and the successful negotiation of meaning between team members depended on a great deal more than just verbal interaction by itself, thus satisfying Wenger's third criterion of a shared repertoire of knowledge and practices. These shared understandings included non-verbal signals, ${ }^{7}$ shared knowledge of work activities, localised terminology, physical objects and processes, knowledge about what had gone before and the role relationships involved, the kind of talk appropriate in each setting and so on.

(1) Shared background knowledge:

Context: Early morning team briefing meeting on factory floor. (Ginette is the TCO).

Ginette: they put [product] twelve point five on the running sheet we do two sizes at twelve point five boxes and bags when youse do these sheets do them properly [sighs]

Marcus: obviously those people who are no good at cheating [voc]

Ginette: fill them out properly I spent two hours yesterday with Isabelle going through the sheets over the last um month and a half and the ones that we did were bloody shocking all bullshit we managed to pack nearly six thousand cases on this line here in three and a half hours [laughter]

Sam: do the do the temps know how to fill them out

Ginette: I don't know no they don't

Sam: no ( )

Ginette: the temps weren't here at three o clock four o'clock

Sam: yeah I know I'm j- I'm just saying it could be

Ginette: that's our people

\footnotetext{
${ }^{7}$ Non-verbal elements of interaction are obviously also very important; the absence of video data (see chapter 4) was an unavoidable limitation on the analysis in this regard.
} 
Example (1) above illustrates how such shared background knowledge enabled team members to understand precisely what was going on in a particular interaction and assisted them in making effective and appropriate contributions in a way that would be quite impossible for an outsider. (The full interactional complexities of the issue referred to in this example will be further explored in chapter 7.)

Although it is possible to infer from this segment of the interaction alone that the TCO is attempting to rectify an apparently longstanding misunderstanding on the part of all or some team members, to really understand the basis of the problem and the way in which this interaction unfolds requires, at a minimum, the following insider knowledge: (i) who Isabelle is (a member of the quality assurance team), (ii) what the running sheets are that Ginette refers to (production logs), (iii) what should be recorded on these (the codes for the correct size, type and number of products packed), and (iv) the expected number of cases to be packed in a given period. In addition, without knowing that line refers to the packing line on which the team worked, and that temps refers to extra people brought in to assist in the physical handling of the cases, it would be unclear whom Ginette is holding responsible. Other background information requiring a degree of in-group knowledge includes the significance of the difference between boxes, bags and crates and the different sizes referred to, as well as the history of this issue and the relative status of different contributors (Ginette was the TCO, Marcus was a stores worker, and Sam was a junior team member who had a track record of making errors like this and being reluctant to acknowledge his mistakes).

This example provides a very clear illustration of the context-embedded nature of (problematic) talk in this team, and provides further evidence that this team fits well within the definition of a community of practice provided by Wenger, Eckert and McConnell-Ginet and others. It also demonstrates the degree to which rich ethnographic description is an essential analytic tool for understanding the significance of a given instance of workplace talk, in particular where the aim is, as here, to identify cases of miscommunication or problematic aspects of workplace talk, and to draw conclusions about their possible sources, trajectory and outcomes.

In the illustrative data analyses which follow, particular attention is paid to three of Wenger's defining characteristics of a community of practice, namely shared ways of (i) engaging in tasks, (ii) sustaining relationships, and (iii) displaying group 
membership. In the case of the Power Rangers team, these were instantiated in a number of specific strategies for the accurate and efficient exchange of vital information and troubleshooting of problems, combined with close attention to relational practice (Fletcher 1999), and a set of characteristic discursive boundarymarking practices such as the distinctive use of humour and group-specific registers already mentioned. The remainder of this chapter sets out to illustrate how, taken together, these shared practices and understandings functioned as important resources for preventing and/or repairing miscommunication and problematic talk, both directly and by building resilience into the team's communicative system.

\subsubsection{Engaging in tasks}

The team members themselves defined communication as being effective or ineffective principally in terms of the accuracy of transmission of information or instructions, or, in their own words, '(not) getting the message across' to coworkers. It is easy to understand why miscommunication at this level would be seen by factory personnel as being of such prime importance: even a relatively minor problem at this level could have negative (and highly visible) practical consequences (cf. Coupland, Wiemann and Giles 1991), incurring real costs in terms of lost production, as well as a loss of 'mana' ${ }^{8}$ in the eyes of co-workers and management.

One example of this which occurred during the pilot study involved a breakdown in communication between the packers and manufacturers about how many tonnes of a particular type of soap powder were to be produced before a scheduled cleanout of the hoppers. Initially a larger run of powder was to be produced and dropped into the packing line hopper than was in fact produced. Nobody had transmitted this information to David in manufacturing who was awaiting advice to change the machinery over for the new product, and had therefore not cleaned the belts to rid the system of old product. When the new run of powder was dropped into the hopper, it was therefore mixed with the previous product, meaning the packing line staff had to re-clean the machinery before the new product could be put through the packing line. Based on the accounts of those involved, this was most probably the result of a simple misunderstanding or mishearing (which was not itself captured on disc), but it resulted in an expensive production 'outage' of several hours.

\footnotetext{
${ }^{8}$ Mana is a Māori word which glosses roughly as "authority, control, influence, prestige, and power or psychic force" (Williams 1971).
} 
One set of interactional and systemic strategies which appeared to be designed to prevent such errors involved making consistent use of routine processes or 'scripts' for conveying or clarifying information and instructions. Embedded in these were other team practices of playful talk and jocular abuse. For instance, the morning briefing meetings always followed a consistent format and provided a regular opportunity for the TCO or others to pass on any important information, to raise questions, issues or problems, and to clarify or reinforce any written instructions or notices. There was also an expectation that people would speak out and air any problems or concerns at these meetings, and this happened often. Secondly, the team also had a clearly understood set of procedures to follow if they were in doubt about what to do, and any member could suggest that a special meeting be called to sort out an ongoing issue or grievance within the team. Thirdly, the TCO or designated leading hands made a practice of going on regular 'rounds' during the course of every shift in order to pick up potential problems and to provide an opportunity for one-to-one clarification or coaching as required. Finally, team members also made use of many different channels of communication, and often backed up important messages by conveying them in two or three different ways. These channels included face-to-face interactions (not always in English), such as team briefing sessions and one-to-one discussions on the factory floor or in the control room, use of the telephone and factory intercom, non-verbal gestures and 'sign language' on the noisy packing floor, and a plethora of written information, including email, notices, posters, instruction manuals, production documentation, computer programmes and so on.

Team members also used some quite specific interactional strategies, with a distinctive flavour, to ensure they conveyed information accurately, or to direct others to do things unambiguously. These included the following simple devices, used singly or in combination: repeating key information, seeking and/or providing feedback, and checking or seeking clarification or confirmation. The three brief examples which follow were all taken from interactions over the intercom linking the manufacturing area where Robert works and the downstairs packing area; their purpose was to coordinate the delivery of the correct amount of powder to the appropriate packing line. 
(2) Repeating key information:

$\begin{array}{ll}\text { Robert } & \text { seven oh one } \\ & \text { seven oh one bro } \\ & \text { seven oh one }\end{array}$

(3) Seeking and/or providing feedback:

$\begin{array}{ll}\text { Robert } & \begin{array}{l}\text { coming up } \\ \text { seven four five } \\ \text { seven four five } \\ \text { copy kiwi? } \\ \text { copy bro }\end{array}\end{array}$

(4) Checking or seeking clarification or confirmation:

$\begin{array}{ll}\text { Robert } & \text { copy Lesia } \\ \text { Lesia } & \text { cool } \\ \text { Robert } & \text { bin } 29 \text { should be your last bin on line one } \\ \text { Richard } & \text { bin } 29 \text { did you say? }\end{array}$

In example (5), where Ginette the team leader was talking to Robert in manufacturing via the intercom, we see the 'safety netting' strategies of repetition, provision of feedback and seeking of clarification in the first three examples combined into a flexible but predictable verbal routine. Note the use of reciprocal 'call signs' (e.g. copy kiwi) and discourse markers (e.g. stand by, yep go), and the routine repetition of numbers, locations and product descriptors (e.g. for the line one orange wave). This also provides a typical illustration of how certain playful aspects of the team culture were embedded into otherwise routine task-oriented interactions, thus giving them a dual function as a way of checking the clarity and accuracy of transmitted information, while at the same time attending to an aspect of team culture and interpersonal relationships.

(5) Use of verbal routines:

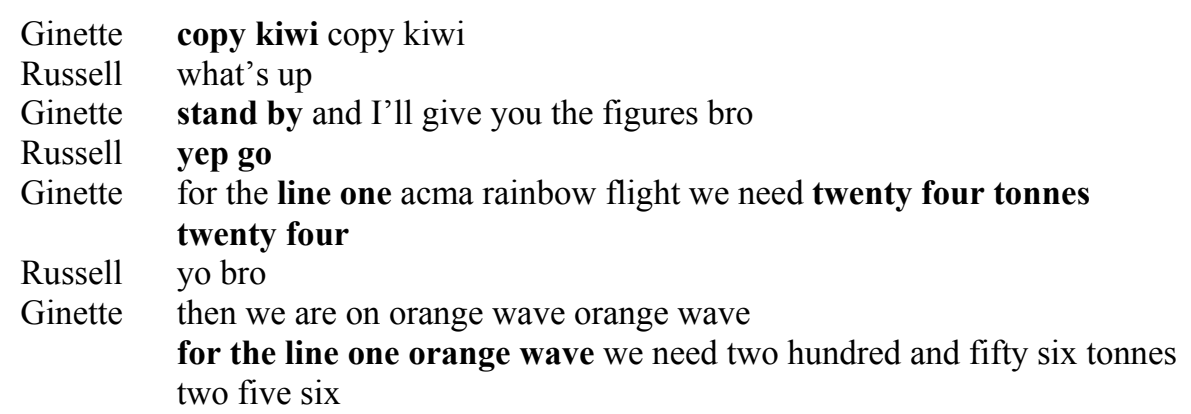

This approximate and slightly tongue in cheek adaptation of stereotypical conventions from the genre of citizens band radio was used by all team members when using the intercom, and had evolved specifically as a humorous technique to prompt the accurate 
relaying of simple information and instructions in this relatively decontextualised and 'noisy' mode of communication. Ginette's use of the nickname kiwi and the familiar and friendly term of address bro when addressing Russell, and his use of bro in return were also characteristic of the way this team interacted. ${ }^{9}$

The next excerpt comes from a very different setting, albeit with a similar basic purpose of 'getting across' information and instructions clearly and efficiently. This was a morning briefing on the packing floor, with Ginette, the TCO, instructing the team in unambiguous terms to correct the procedural error some individuals had been making as already seen in example (1) above. The strategy Ginette used here was to be extremely direct and explicit about the precise nature and implications of the error, whilst at the same time refraining from directly criticising any individual team member, and, as indicated by the team's laughter, successfully sweetening the pill via an exaggeratedly humorous delivery.

(6) Being very direct and explicit:

Ginette you must fill them out properly the purpose of these sheets is to give information for people up there on how these- the efficiencies of these lines when we fill out a sheet that says we nearly packed six thousand cases in threethree and a half hours that's a load of shit that's running the machine at five hundred packets a minute ... fill them out properly [general laughter]

\subsubsection{Maintaining relationships}

The data provided further evidence of different kinds that team members would pursue their practical or transactional goals in a very focused way (e.g. conveying information, giving instructions, complaining, problem-solving etc), while at the same time taking great care to avoid creating misunderstandings or bad feeling at an interpersonal level, or conversely designing their interactions to proactively build solidarity with their workmates. This was achieved by using strategies which functioned to maintain positive relationships, motivate others and reinforce a positive team ethos. In excerpt (6) above, for instance, as well as conveying information and instructions, the TCO was simultaneously attending to important task-related and social/relational objectives. Although the details of content and setting could not be

\footnotetext{
${ }^{9}$ Although bro is technically an abbreviation of brother, and therefore more commonly used as a solidarity marker between males in New Zealand English, it was not unusual for bro and brother to be used by both males and females in the Power Rangers team to address the four women team members, as is increasingly common in other Māori and Polynesian contexts (e.g. King 1999).
} 
more different, the deft way Ginette dealt here with the potentially problematic threat to face implicit in her strongly worded assessment of the team's performance strongly echoes the strategies we saw being used by Jan in her complaint to Heke about his staff member's under-performance in chapter 5.

In excerpt (7) below, we see how Lesia, who had recently been nominated by Ginette to take on the role of leading hand in her absence, made subtle use of various discourse strategies to attempt to stay 'on side' with an equally experienced coworker in the course of pursuing his assigned practical objective of monitoring the quality of her work on the packing line, thus proactively preventing any possible misunderstanding of his attitude towards her. (Contextual information is in italics)

(7) Softening authority:

$\begin{array}{ll}\text { Lesia } & \text { what's the speed [referring to the conveyor belt on the packing line] } \\ \text { Sue } & \text { speed slow one twenty [number refers to a dial reading] } \\ \text { Lesia } & \text { thanks a lot [4 second pause] } \\ \text { Sue } & \text { hurry up [addressed to a third party] } \\ \text { Lesia } & \text { got no idea eh brother [5 second pause] } \\ & \text { keep 'em eyes on the rejects [sub-standard packaging] } \\ & \text { keep 'em eyes on the rejects er sue please and also on your weight } \\ & \text { [refers to a running joke amongst the team about diets/losing weight] }\end{array}$

Lesia opened the exchange by asking Sue a neutral question about the conveyor speed before tactfully reminding her to watch out for 'rejects' or substandard packaging. Lesia used explicitly polite language (thanks a lot and please), aligned with Sue's indirect complaint of hurry up (addressed to another worker) with a supportive statement coupled with a familiar term of address (got no idea eh brother) and gently teased Sue (who was actually quite slender) with his reference to a current running in-joke about diets. In this brief exchange then, Lesia used no fewer than five separate discourse strategies to neutralise the status difference implied by his giving instructions to her (which is the problematic aspect here), and his friendly, unhurried tone of voice in this interaction added to this overall effect.

The next excerpt involved a greater degree of joint negotiation, and the strategic use of indirect linguistic forms and strategies. Helena had asked Chester if there was any more glue, as they had run out on her packing line. He offered her some glue, but she was not sure if it was the right formula. 
(8) Indirect request

Chester well we may not have any glue then coming in

Helena that's why I need to know if all those buckets are ours no one else uses those eh on site?

Chester well if they're there you need glue use it they're not reserved for anybody

Helena yeah but it might be the wrong glue

Clearly this was not a case where either participant was simply conveying information; rather they were jointly engaged in solving Helena's problem, which meant Chester having to take time out from his own work. Example 8 shows how Helena made careful use of justification (that's why I need to know, no one else uses those) and conditional, attenuated formulations (if all those buckets are ours, no one else uses those eh on site?, it might be the wrong glue) to question Chester, ask for the buckets of glue, and challenge the answer he gave her, instead of using more direct strategies which might have made him disinclined to cooperate. Note also the use here of the addressee-oriented endtag $e h$ which, like bro in the earlier examples, functions as a solidarity marker in New Zealand English.

The finely-tuned attention in these examples to the interpersonal aspects of their interactions, closely and skilfully interwoven with the transactional business at hand, was typical of the patterns of communication in this team. This set of practices assisted team members in dealing with potentially problematic aspects of the current talk-in-interaction (e.g. threats to face). They also clearly played a vital part in building and maintaining good relationships, thus providing a baseline of goodwill which provided a degree of insurance against interpersonal misunderstandings in other situations where a degree of unvarnished straight talking might be required, or where conflict of some kind arose.

\subsubsection{Displaying group membership}

The attention paid to the interpersonal aspects of their interaction clearly had a very positive impact on the Power Rangers' motivation and team spirit. In fact, the most noticeable characteristic of this team was an exceptionally strong orientation to group morale and to their collective identity within the factory. One of the most remarked upon aspects of this team's culture was their unique and light hearted, but rather politically incorrect and irreverent style of interaction. Their particular blend of ingroup verbal humour and practical jokes, combined with a steady diet of sarcastic banter, teasing, jocular abuse and 'blue' language, was arguably the team's defining 
characteristic as a community of practice, and a prime marker of their group identity. This final section provides some examples of how this humorously contestive interactional style related to the occurrence and management of miscommunication and problematic talk within the Power Rangers team.

\section{Humour and jocular abuse as a marker of team solidarity}

The overarching function of both the collaborative and contestive forms of humour (cf. Holmes and Marra 2002) observed in the interactional data appeared to be as a means of constructing group solidarity and marking the boundary between the Power Rangers and other teams and groups in the factory. This was reinforced by comments made to the researchers by many team members themselves, and also by other factory staff who remarked on how their characteristic use of humour and banter set the Power Rangers apart as a 'tight team'. It is not a coincidence that whilst their antics were a constant source of amusement to others in the factory, the in-group nature of this team's interactions not infrequently also made others feel excluded or uncomfortable. The team's use of humour was one aspect of a shared repertoire of resources which enabled members to communicate in a kind of verbal shorthand which could be difficult for outsiders to interpret or break into. This is also a clear example of the point made in chapter 3 (and again in chapter 5), that apparently miscommunicative talk can in fact promote desirable outcomes, and that something that is assessed as problematic in one context may function as an example of effective communication in another, depending on the perspective from which it is viewed.

Excerpt (9) provides a typical example. (See also excerpts (10) and (11) below.) Here David and Peter, two of the manufacturers, and Tony, a packer, were having a threeway exchange over the intercom to coordinate the delivery of a batch of powder to the packing line conveyer belt. The usual tongue in cheek allusion to $\mathrm{CB}$ radio conventions was present here (copy peter, one point three there), but the task and medium also provided an excuse for a bit of light-hearted verbal play. David was a Pakeha New Zealander and Peter was Tongan, but the use of my nigger (in defiance of official factory policy on racist language and verbal harassment) was perceived as a friendly address term in regular use amongst core members of the team, as we can clearly see from Peter's exaggeratedly warm response. This brief exchange was also literally packed with positive politeness devices, mostly in the first three lines before 
the men got down to the task at hand, something which contributed to the overall humorous effect. There were no fewer than five uses of high-solidarity address terms (bro, brother, my nigger) in addition to the use of Peter's first name, along with the repeated positive evaluation sweet as, and Peter's explicit thanks and compliment (you are the best) to David.

(9) Jocular abuse and exaggerated solidarity

Context: Manufacturers and packers talk over the intercom:

David: $\quad b d$ three ninety nine my nigger

Peter: thank you brother you are the best bro ...

David: $\quad$ sweet as bro sweet as

Tony: copy peter is the [product]coming down

Peter: yeah bro one point three there

David: did you ring up Ginette and tell her

There was a clear in-group/out group boundary apparent between the Power Rangers team and other teams and work groups within the factory. Language and modes of interaction which were considered acceptable within the team would usually not be tolerable outside of it, and in the 'wrong' context, would most probably be seen as highly problematic and miscommunicative, possibly attracting intervention from management at an official level if they were seen to be in serious breach of extant codes of conduct within the factory. The fact that the targets and initiators of the most extreme forms of humour and jocular abuse tended also to be the core, most well-integrated members of the team, is diagnostic of in-group solidarity being the prime function of this set of interactional practices. In the words of the TCO, "our team created a culture that we were all comfortable with ... when someone new joined us, we obviously took the path of easing them into our culture", and the use of expletives and jocular abuse by team members "was a 'we know each other well' thing ... no one really took offence". ${ }^{10}$

The observational and interactional case data revealed this pattern quite unequivocally: the off colour jokes and banter incorporating sexist, racist or personally offensive remarks, and engagement in overtly contestive interaction, unexceptional 'in-house' amongst members of the Power Rangers team, were not in general carried into work conversations with non-team members. Team members

\footnotetext{
${ }^{10}$ A fuller discussion of this aspect of the team's interactional style, in particular the use of expletives as solidarity signals in face-threatening acts such as complaints and refusals, can be found in Daly et al (2003).
} 
were also consciously aware that they used what they termed 'different vocabulary' depending on whether their interlocutor was a team member or not. This point was repeatedly commented on in interviews with team members, and is also nicely captured in the two contrasting data excerpts below. In example (10) the TCO, Ginette, assertively refused a request by Russell, one of the packers (and a fellow Samoan), to go and fetch a piece of equipment, to which he responded in typical no holds barred Power Rangers style.

(10) Refusing a request (a)

$\begin{array}{ll}\text { Russell } & \text { can you get me one please [in Samoan] :fa'amolemole: [please] } \\ \text { Ginette } & \text { you get one } \\ \text { Russell } & \text { ah you're not doing anything } \\ \text { Ginette } & \text { you go and get one } \\ \text { Russell } & \text { fuck it }+++ \text { fuck you go get your fucking legs out here (fatters) } \\ \text { Ginette } & \text { why didn't you get one before I talked to you about that yesterday } \\ \text { Russell } & \text { because we're busy + I got to get all that out of the way }\end{array}$

On superficial analysis Russell's initial request can you get me one please fa'amolemole was expressed politely with the please repeated in Samoan. The confrontational and apparently disrespectful way he then reacted to Ginette's repeated assertive refusals (you get one, you go and get one) is not at all what would be conventionally expected in a workplace, let alone in an interaction between a worker and a manager, and on the face of it should be analysed as a clear example of problematic talk at an affective and/or relational level, as Russell appeared to be making a personally offensive remark, as well as overtly contesting Ginette's status as team leader. However, this exchange was entirely consistent with the interactional style of the Power Rangers team, as described above, and as such was in fact reflective of the close relationship that we know from the ethnographic data existed between these team members. If Russell's opening request were interpreted as being tongue in cheek, rather than as a literal polite request then this sequence would take on the character of co-workers acting out a playful script, although it may well have had a contestive, serious edge to it as well, as seen in Ginette's complaint why didn't you get one before I talked to you about that yesterday.

This indexes a problematic matter at the task level as well as in the negotiation of relative status. This interpretation is supported by the way in which Ginette did not react to the increasing escalation of Russell's apparent insubordination and jocular abuse. By keeping her contributions neutral, she was arguably maintaining a degree 
of distance and asserting her status and ability to deny the request. However, by the same token, she obviously did not take exception to Russell's expletive-laden demand in and of itself. Note also how the running joke about people's weight problems, as seen previously in example (7), also surfaced in this interaction in Russell's use of fatters to address Ginette, which is once again an indication of the playful nature of this seemingly conflictual sequence. It also worth noting that the very upfront 'ribbing' we see in this and other examples is also typical of certain styles of repartee in informal contexts within traditional Samoan culture (Alfred Hunkin, personal communication).

The tone of the interaction in example (11) was completely different. Ginette and Francie, a rework coordinator from the quality assurance department, were sorting out a problem with some boxes of product that were not up to standard.

(11) Refusing a request (b)

\begin{tabular}{|c|c|}
\hline Francie & do you have an $\mathrm{NCR} 7^{11}$ for that (boxes) over there \\
\hline Ginette & yeah I’ve I'm waiting for a number ++ \\
\hline & I need to see Vicky about the NCR thing \\
\hline & I haven't got a number for it yet \\
\hline Francie & oh how would you get it \\
\hline Ginette & when I get to see Vicky +++ \\
\hline Francie & $\begin{array}{l}\text { oh hows about you just give it to me now }+ \\
\text { take a copy of that }+ \text { so I can compare it } \\
\text { and I'll take the number then }+++\end{array}$ \\
\hline Ginette & (where are they) + do you want it right now \\
\hline Francie & if it's possible [laughs] \\
\hline Ginette & $\begin{array}{l}\text { it's just I've left a- + I've got- um Jennifer's working + } \\
\text { going through it as well }\end{array}$ \\
\hline Francie & oh okay is it possible tomorrow then \\
\hline Ginette & I'll get it to you tomorrow morning yeah \\
\hline
\end{tabular}

By contrast with her bald response to Russell in example (9), here, after an initial brief acknowledgement (yeah), Ginette immediately justified her refusal of Francie's initial request for a copy of the form by means of a rather full explanation I've I'm waiting for a number I need to see Vicky about the NCR thing I haven't got a number for it yet. Francie did not accept this initial refusal to comply with her request, as seen in her three further hedged attempts to elicit a positive response; Ginette was also obviously reluctant to comply, as shown by her continued resistance to Francie's persistence with her request. However, both women maintained a tone of respectful negotiation, avoiding direct disagreement, before eventually negotiating a

\footnotetext{
${ }^{11}$ A NCR7 is a Non Conformance Report, or a sheet filled out when a product is not up to standard.
} 
compromise solution. These two women had worked together for ten years, and clearly had a friendly relationship, but even so, it was quite evident from the style of their interaction that Francie was not a member of the Power Rangers team, and that the style of interaction typical of that team was not just a reflection of a wider factory culture. (See chapter 7 for examples where Ginette does use the typical Power Rangers style when talking with female team members).

To relate the analysis of these examples back to the main topic at hand, it is likely that this interaction would have been considered by Ginette to be more problematic than her exchange with Russell in the previous example. On the basis of a close analysis of the recordings alone, it is clear that this interaction required a lot more interactional work on Ginette's part to get an outcome that was acceptable to her without risk of damage to her relationship with Francie (and vice versa), and there is also clear interactional evidence of goal conflict between the two women, with Francie attempting to gain Ginette's immediate compliance with a request, and Ginette resisting this. However, it is probable that any analyst presented with these two excerpts side by side, with no supporting ethnographic data, would far more readily classify the exchange with Russell as an example of problematic talk on the basis of its apparently confrontational tone and content alone, let alone with the added knowledge of the status difference between Ginette and Russell. However, having done a thorough analysis of how the Power Rangers team functions as a community of practice, we can instead entertain the opposite, counter-intuitive possibility that this is in fact an example of effective dialogue. These two examples thus demonstrate very clearly the crucial importance of interpreting data snapshots such as these in the context of the interactional practices and team culture within which they are framed. They also reinforce the points made in previous chapters that apparently miscommunicative talk can have a positive function, and apparently unproblematic talk may be "deceptively adequate" (Coupland, Wiemann and Giles 1991).

\section{Humour as a resource for managing problematic talk}

In addition to its importance in constructing and maintaining a strong sense of group identity, as already noted, the Power Rangers' culture of humorous interaction also generated a baseline resource of underlying goodwill and ease in the team's interpersonal relationships which meant that actually problematic interactions might 
be less likely to escalate into a full-scale episode of misunderstanding or conflict, and/or would potentially be easier to resolve. As will be elaborated further in the next chapter, the TCO actively fostered and participated in this aspect of team culture, and viewed it as a very important vehicle for creating positive relationships and effective communication within the team, and for helping maintain morale by countering the inevitable tedium and physical discomfort of factory work. Ginette did her best to ensure that the Power Rangers team provided a happy and positive social environment for its members, although this was never allowed to be at the expense of the work itself. Her stated intention was to create a climate that encouraged humorous interaction as a seamless part of the working day, as suggested in these excerpts from an interview with Ginette and another staff member:

\section{(12) Interview with Ginette:}

There's all sorts of different humour ... types where you can have a joke amongst yourselves, You have to be cautious ... of course some people haven't got a sense of humour at all so you've got to be cautious with what you tell, work jokes or what ever, or it could be ... something silly that you could do to another person, or also taking the mickey out of management which ... always draws a good laugh at the end of the day. But it's all good stuff ... it could be anybody walking past, it could be something that we heard on the radio, it could have been something somebody did on their days off that we ... find really funny, or a mistake, that always draws a lot of good humour, when somebody makes a big blunder.

(13) Interview with John:

There's no doubt that having humour in the workplace, people are much more relaxed (get) much more enjoyment out of what they're doing and it takes the mind off uh the boredom and monotony of just sitting there and looking at each other all day. So yeah, I think Ginette's right ... it's an excellent work safety record we have here. The thing is that you can have people joking around here, and if anybody sees anything that's not right that'll stop straight away. And the interesting thing is, once everything's tidied up, if there was a spillage or anything like that, then they're back to having the joke where they left off last time. So very conscious about their work environment, but very conscious of having a good time with it as well. So it's good.

As we have also seen to an extent already in earlier examples, humour was used extensively in this team as a localised discourse strategy for downplaying or challenging authority, defusing tension, or softening negatively affective speech acts such as criticism, complaints or refusals. As such, it often had a dual function, working as a softener or mitigator at the local level of the interaction, as well as more globally as a means of indexing the collective team ethos and identity. This distinctive mix of humour and bald on record face threatening acts (Brown and Levinson 1978, 1987) represented a very distinctive interactional style which depended for its acceptability and success on high-trust relationships between all team members. The next two examples from the team's early morning briefing 
sessions provide further brief illustrations of this highly typical discursive practice. In each case we see Ginette's strategy for dealing with the fact that everyone is not yet present, an intractable problem which had been an ongoing irritant (see Table 6.1 above). In (14), the meeting was already well under way when Sue arrived late, interrupting Helena's briefing of the team.

(14) Late for work (a)

$\begin{array}{ll}\text { Helena } & \text { there's run upon run upon run so that's I'd do at least five or six } \\ \text { Ginette } & \text { good afternoon Sue } \\ \text { Lesia } & \text { good afternoon Sue } \\ \text { Sue: } & \text { hi everybody I'm here }\end{array}$

Ginette's sarcastic greeting, delivered with a smile, was immediately echoed by Lesia, a leading hand in the team who had recently been appointed to deputise for Ginette at times when she was absent. Sue's cheerful riposte shows she took the implicit reprimand in good part. However, a public criticism of an individual team member in relation to a work matter, however light handed, was unusual, so Ginette could be reasonably sure that her intended 'message' had been received, not only by Sue, but also by other members of the team who had been becoming increasingly 'slack' about getting to work on time. The next example is from yet another early morning briefing, where this time only five team members were actually present as Ginette called the meeting to order.

(15) Late for work (b)

Ginette good morning everybody it's just lovely to see you all this morning + just can't imagine my life coming into work not seeing you every day + nice to see you all well

All [general laughter and a range of good-humoured responses]

Ginette one one three +++ nice to see everybody's here on time +++

As people gradually drifted in, Ginette continued to comment on their tardiness in a similarly ironic vein to example (14) above. She used humour very effectively to maintain attention, creating a sparky, engaged interactive style, while simultaneously reinforcing the expectation that everyone should arrive on time for morning briefings. She then ended the meeting with a humorous threat that if the team did not complete the scheduled production run by the end of the working day, they would have to stay later to finish it. Manny offered an ironic riposte, which was quickly turned by Lesia into a gentle tease of Russell, his fellow shift planner, whose reputation as 'a bit of a dreamer' at times raised a laugh, thus relieving any tension that may have built up. 
(16) Late for work (c)

Ginette if you don't finish it by six o clock you're staying here until you do finish it ++ that a good deal

Manny that's good news give me the bad news now ...

Lesia the bad news is that Russell is a liability for all of us [all laugh]

\section{Social humour}

More extended humour sequences such as telling jokes or teasing also occurred in their own right, as in the following excerpt (17) from a lengthy interlude during which Peter's co-workers are teasing him during a period of 'downtime'. Despite (or perhaps because of) their 'in your face' and often contestive nature, such interactions clearly also provided an additional opportunity for maintaining positive interpersonal relationships. These humour sequences again often involved jocular abuse or teasing focusing on personal characteristics. This next example once again picks up the running joke about people's weight which repeatedly surfaced in interactions throughout the team at this time, here in conjunction with a discussion about a news item about a war to which New Zealand was reported to be sending troops.

(17) Social humour (a)

\begin{tabular}{|c|c|}
\hline \multirow[t]{2}{*}{ Peter } & oh man I'm starving I am starving \\
\hline & I might go and join the war remind me of the old days the army and the front row \\
\hline David & you'd be the first one to get shot \\
\hline Peter & why //what makes $\backslash$ you say that \\
\hline David & $\begin{array}{l}\text { /you're sol\ } \\
\text { you're so big }\end{array}$ \\
\hline Peter & brother [warningly] \\
\hline David & $\begin{array}{l}\text { it's very rare that a bullet will miss you } \\
\text { [laughter] }\end{array}$ \\
\hline Peter & yes //that's not on $\backslash$ \\
\hline David & / look at the $\mid I$ size of your stomach \\
\hline Peter & that's NOT on $(3)$ \\
\hline David & $\begin{array}{l}\text { actually they'll close their eyes and sh- fire a shot } \\
\text { [laughter] }\end{array}$ \\
\hline Peter & [drawls]: oh: I see \\
\hline David & they got no problem missing that \\
\hline
\end{tabular}

This type of humour functioned as a type of social talk, akin to gossip and discussion of sports matches. It tended to occur in the gaps between tasks and therefore did not interfere with the demands of the job. However it definitely helped fight boredom, thus maintaining morale and keeping people 'on their toes' as Ginette put it. 
In the next and final excerpt, we see how the shared history of the dirty hopper incident described earlier and the verbal routines associated with the intercom system were invoked as a resource for a co-constructed humour sequence following a minor instance of misaligned communication between Dennis and Russell.

(18) Social humour (b)

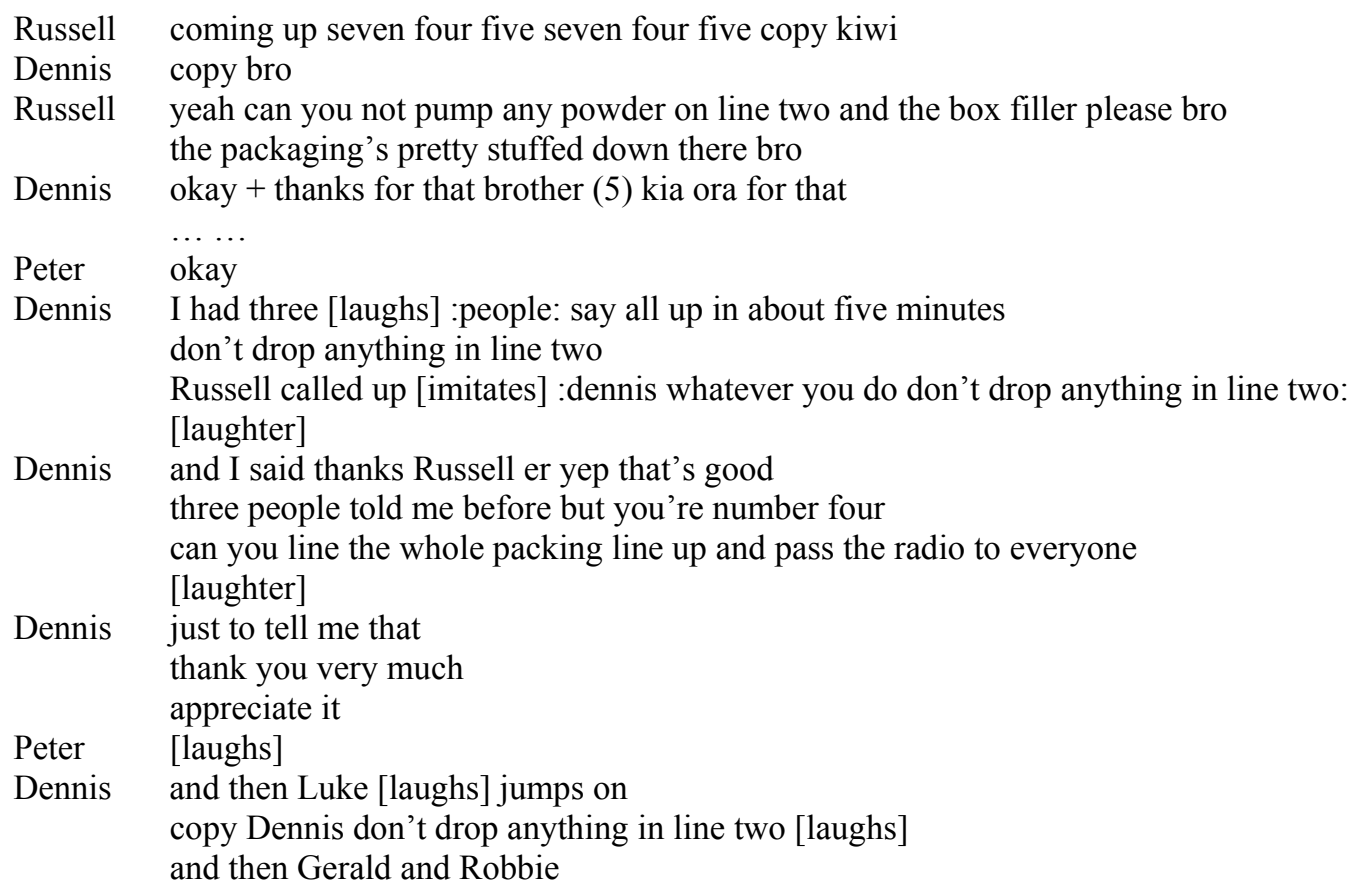

It was also quite common, when things were running smoothly and there was a bit of 'downtime' for team members to engage in some light hearted skylarking and practical jokes. Again, this was primarily social humour, but it also reflected a degree of largely good-natured rivalry between the packers and the manufacturers which could on occasion flare up into a genuine dispute or practical misunderstanding. For instance, in their own words, the packers might 'get on the radio and rark up' the NSD staff (i.e. the manufacturers), something which they described as just joking and playing silly buggers'. At such times, the radio interaction would 'get silly'. For instance, the workers might copy exchanges they had heard on television (roger and out, copy charlie one), or send spurious messages such as something isn't right with mana, might be the clutch, might be the hydro-electrics (mana being a machine which has no clutch or hydro-electrics). Generally this did not cause any actual miscommunication, as the faux routines were so obviously spoofs, but sometimes people were taken in, and hurt feelings, irritation or, very rarely, a negative impact on the workflow could result. This social humour therefore also had its dark side. 


\subsection{Conclusion}

This chapter has presented the first part of a complex case study of a factory team, the Power Rangers, utilising an interactional sociolinguistics analytic approach to explore in detail the intertextual patterns of communication in this team within a discursive community of practice framework. In this chapter I have described and analysed the general communication patterns in this team, identified the communication challenges they faced and have begun to examine the discursive strategies members of the team had at their disposal to manage the occurrences of miscommunication and problematic talk that inevitably arose in their daily working lives. We have seen how the team's shared orientation to the tasks at hand was mediated through a unique set of discursive practices which provided a rich resource for creating a positive team environment and constructively negotiating the many practical and interpersonal issues that could arise in the course of a typical working day.

So far, this case study has demonstrated that the methodological framework used is a robust one. It offers greater explanatory power than an analysis of isolated interactions or analyses restricted to very specific aspects of miscommunication or problematic talk, because it opens more of the true complexity of (mis)communication in workplace settings to analytic view. The analytic approach has combined selective micro-level interactional analysis with an in-depth ethnographic survey across the data set to produce a thick description based on the situated practices of this team and on information about the complex adaptive system within which it operates.

One clear advantage of this multi-pronged approach is that instances of miscommunication and problematic talk that would undoubtedly have been missed or mis-classified using a more uni-dimensional approach were able to be identified in this data set, and could be interpreted in context. It should also be clear from the findings presented in this chapter that being able to combine rich ethnographic data of various kinds with the ability to track issues and people through related longitudinal sets of recorded interactions has produced some unique insights into the nature and processes of miscommunication and problematic talk. This analytic theme 
is further developed in Chapter 7, which turns to a consideration of the way the team coordinator uses her own discursive repertoire within the framework of the team's unique culture and practice to 'manage the contradictions' of communication that are inherent in her role. 


\title{
7 Managing the contradictions The discursive strategies of the team leader
}

\author{
The TCO instructing packers on how to stack boxes correctly: \\ Ginette: when yous take them off stack them properly \\ David: $\quad$ oh //so who was | taking them off from here and loading it \\ Russell: $\quad$ lit was [name]\|I I always stack back on \\ Sam: [indignant tone] :you're assuming eh assuming bullshit eh: \\ Ginette: $\quad$ no I said what did I say + I said when you take them off stack \\ Sam: \\ them properly that wasn't an a-assumption // that was an I \\ somebody was not //stacking it properly 1
Ginette: $\quad$ /that was- that was anll instruction
}

\subsection{Introduction}

The previous chapter provided an introduction to the unique dynamics of the Power Rangers team as they worked their shifts over two periods spanning several months, and described the challenging communication and systemic issues that were occupational hazards of their daily working lives. Examples of the kinds of miscommunication and problematic talk that typically arose in team members' interactions, and the discursive strategies they used to manage these issues, were then analysed at a number of different levels within a discursive community of practice framework. The multi-pronged 'thick description' that came out of this combined ethnographic and interactional analysis is the basis for the second half of the case study presented in this chapter. Here we move away from a consideration of the team as a whole, to a focus on the discursive strategies and interactional devices used by Ginette, the team coordinator (TCO), to balance different and sometimes competing communicative demands as she and others dealt with the many problematic issues that typically arose in the course of a shift.

This part of the case study continues to build on the theoretical and analytic framework introduced in the last chapter, but enriches the analysis by extending it in 
fresh directions and drawing in an integrated way on the relevant theoretical constructs, analytic tools and dimensions from all sections of the working model presented in chapter 5. Firstly, a different perspective on the data is achieved by tracking a series of related interactions to explore the ways in which selected instances of miscommunication and problematic talk unfolded and were managed intertextually by a varying cast of participants. Secondly, an explicitly critical dimension is added to the analysis by exploring the interfaces between miscommunication, power and social identity in the TCO's discourse. This demonstrates the general utility of adding another set of analytic resources and perspectives to the 'toolkit' approach, as proposed in chapter 5, and also provides important insights into how the TCO's wide discursive and linguistic repertoire helped her to balance (and sometimes exploit) the inherent contradictions in her role as she attempted to optimise communication within the Power Rangers team.

The next section (7.2) draws from the ethnographic case data to profile the team coordinator, and outlines the particular challenges she faced in her role, together with the broad management principles or strategies to which she subscribed and which influenced her own communicative practices. Section 7.3 uses a discursive form of critical incident analysis to trace a particular misunderstanding that occurred through a series of thematically-linked problematic interactions. This analysis serves to highlight the extent to which Ginette's personal ethos and practices as TCO influenced the specific discursive patterns and strategies observed within the team, and further illustrates how the communicative culture of this community of practice played itself out in interactional terms across different genres and settings. The results of a different kind of data tracking are reported in section 7.4. As well as allowing recurring topics and themes to be identified and analysed across the data set, the research design also involved 'wiring up' certain individuals in order to record their interactions as they moved through their working day. Different team members were tracked for varying periods of time, but Ginette was followed and the majority of her interactions were recorded in this way throughout the data collection period due to her key position in the team (see chapter 4). A critical discourse analysis of these interactions between Ginette and her co-workers allows us to gain insight into the way Ginette routinely constructed and maintained different aspects of 
her personal and social identity, and shows how these discursive practices in their turn related to her management of miscommunication and problematic talk.

\subsection{Ethnographic overview: the TCO}

\subsubsection{Personal profile and role}

The coordinator of the Power Rangers team at the time of this case study was Ginette Tolai ${ }^{1}$, a feisty and outspoken Samoan-born woman in her mid-30s who had migrated to New Zealand in her late teens. She was fully bilingual in Samoan and English, had a smattering of other Polynesian languages including Tongan and Maori, and, although English was not her mother tongue, her competence in spoken and written English were indistinguishable from that of any New Zealand-born native speaker. Ginette had been a member of the Power Rangers team for a number of years after transferring from one of the night shift teams. She was the only Samoan speaker in the Power Rangers for some time, but over a period of time the five other Samoans in the team had also transferred to join her there.

Ginette had a reputation in the factory as an exceptionally capable and effective team leader, a determined 'straight talker' who was respected by workers and management alike as someone who 'pulled no punches' when it came to meeting production and quality targets. Her personal ethos was reflected in her reputation for integrity and hard work, and in her orientation to community and family values. She was known to be very committed both to the company and to her own team, and was also proactive in her support (both personal and professional) of the Samoan and other Pacific Island workers at the factory, especially those with limited English proficiency or educational qualifications. She was acknowledged as being a team player who supported her colleagues, regularly socialised with them, and who was always prepared to 'have a laugh', often instigating the fun herself, as we saw in the interview excerpt in the previous chapter.

As already outlined in chapter 6, all the teams at this factory were characterised as 'self-managing'. Each team had a coordinator selected from within its own ranks, and there was a comprehensive training matrix in place to encourage staff in

\footnotetext{
${ }^{1}$ As in previous chapters, all names for people, groups and places used here are pseudonyms.
} 
upskilling and taking on various leadership roles. The management structure was therefore reasonably flat. Ginette herself reported to the powders department manager, but her role was otherwise relatively autonomous. Her responsibilities included overseeing and coordinating the team's production and quality processes, managing staffing issues (including training and development needs) and liaising with other factory staff and management as required. Because she was seen as very capable and was prepared to speak her mind, she was often asked for her opinion on important issues (e.g. the new training matrix). She was also a regular speaker at the new staff induction sessions on teamwork and the organisation of factory staff.

Not surprisingly, Ginette's job was a very busy one and her days were extremely long. The regular shift times were $6 \mathrm{am}$ to $6 \mathrm{pm}$, starting with a briefing meeting of all the packing line staff to cover what was to happen during the shift, and to discuss the current production run or any interpersonal or team management issues. Ginette routinely came in up to half an hour early, especially on the first day of the four day shift, in order to check her email and liaise with the night shift staff, to ascertain what the Power Rangers team had to do for the upcoming shift, and whether there were any problems or issues to be dealt with at the team briefing. A significant proportion of her time was spent on communication and liaison of various kinds both within the team, and between the team and the rest of the factory. This occurred via face to face meetings and informal interactions, as well extensive use of telephones, radio and intercom systems. Email was seldom used within the team, but was an important medium of communication amongst team leaders and also between team leaders and managers at department and factory level.

\subsubsection{Management style and strategies}

Ginette had a well-developed personal management style and philosophy, and clearly articulated goals and aspirations for the team. Although she had no formal management training as such, she clearly understood and was able to apply the organisational principles of teamwork and continuous quality improvement that were part of the overall ethos in the factory. Her drive and pride in her work were reflected in a great attention to detail and a relentless concern with the efficiency and quality of the production process. She was an acute observer of people and processes, and made a practice of critical reflection and evaluation which fed back into ongoing improvements and refinements of systems and processes within the team. 
An important aspect of Ginette's management style was her encouragement of team members to take responsibility for and ownership of their work and to participate in decision making. This was obvious from the way team members felt free to speak out at briefing meetings, to challenge one another's actions and opinions (including Ginette's) on the factory floor, and to take the initiative in solving the various problems they encountered. Ginette kept a close overview and ran a 'tight ship', and, as we will see below, could be quite explicitly directive at times, but her prevailing objective was one of fostering collaboration and autonomy within the team. She thus delegated many day-to-day management tasks, and actively mentored and trained team members to take on additional responsibilities or to learn new tasks if they showed interest or aptitude. However, she tended to retain control over personnel and training matters, and any 'tricky issues' (relational or work-related), as she viewed these as being her prime responsibility as team leader. Thus, for example, quality assurance was part of Helena's role, and shift planning on the packing line was delegated to Lesia and Russell, but with the proviso that if there were major problems, Ginette would be involved in their resolution.

Optimising communication (both task-based and relational) was seen by Ginette as absolutely fundamental to the success of the team and its individual members. A range of strategies was employed to minimise the likelihood of miscommunication. For example, as we saw in chapter 6 , a number of routine practices and procedures were well-established within the team, including regular briefing meetings and protocols for communicating via telephone and radio. At a systems level, there was a lot of redundancy built into the communications network, with multiple telephones, radios, and forms of documentation supplementing face to face interaction, and Ginette exploited this range of options to good effect. For example, she routinely conveyed the same information via a number of different channels and settings, and produced diagrammatic and plain English versions of technical manuals and safety instructions to assist staff who had limited literacy or language proficiency. She also made regular 'rounds' of the packing line, liaised closely with the manufacturers upstairs and maintained a regular presence in social contexts such as the smoko room as another way of checking information and instructions had been received and understood, and generally 'taking the pulse' of the team dynamics. 
Although English was the main language of communication, the fact that nearly a third of the team spoke or understood Samoan provided Ginette with an additional resource to facilitate social aspects of interaction or to 'scaffold' the learning or understanding of technical English in this setting (Holmes and Stubbe 2004). For instance, she reported that she might introduce an important new concept or technical term in Samoan first, then move to a mix of Samoan and English until she was certain mutual understanding had been achieved, a strategy that was also directly observable in several instances. She was however also conscious of the risk that this kind of bilingual interaction had the potential to be divisive of the team if not sensitively handled.

Finally, Ginette was also very proactive in nurturing a positive team culture. As we saw in the previous chapter, the team's humorous banter and social talk was an important component of this, and Ginette not only participated in this, but actively promoted it. She was also sensitive to any undercurrents of interpersonal conflict or discomfort, acting promptly to deal with any such problems and encouraging team members to speak out if they had a grievance. A diagram on her office wall, 'Ginette's Triangle: Team-Individual-Task', nicely summed up her philosophy that people would be motivated to perform better, individually and as a team, if they felt they were in a safe and supportive environment. This would be one where they could 'have some fun or a bit of a chinwag', where plain speaking was well tolerated, and any seeds of discontent would therefore fall on less fertile ground. The next section analyses how some of these management principles and strategies played out in an actual case of miscommunication embedded in a linked series of problematic interactions.

\subsection{Discursive management of a problematic incident}

In the previous chapter, we saw several examples of team members working through an interaction that was problematic in some respect. The wider data set also included many instances where the process of negotiation and clarification was much more complex than in these cases, often extending well beyond the boundaries of a single interaction as a particular issue or task was followed through a series of related interactions by staff members during the course of a shift, or even across successive shifts. In this section we follow an issue across the boundaries of different contexts 
and interactions to illustrate how complex even the simplest misunderstanding can be in interactional terms when it is looked at intertextually and in the context of the discursive and other practices within which it is embedded. We will also see how Ginette's discursive and management practices as described above influenced the ways in which the problematic talk unfolded and was dealt with. Finally, the analysis highlights the tension that can exist between relational goals (e.g. motivation, morale, team spirit, saving face) and transactional goals (e.g. giving instructions, criticising, coaching). Brief excerpts from this incident were included as part of the community of practice analysis in chapter 6 , but are analysed here from a different perspective.

This incident related to an ongoing problem: some of the packers had been making mistakes with entering the packing codes onto the running sheet. This had created some serious delays for two other shifts of workers over the past week, as well as creating confusion in the stores and quality departments. Ginette had expressed her concern to one of the researchers that she was 'not getting the message across' to one or two individuals in the team in particular. The first excerpt comes from an early morning briefing meeting shortly after this, where Ginette was telling the team in no uncertain terms that there was a serious problem that needed to be addressed.

(1) Problem with the packing codes (a)

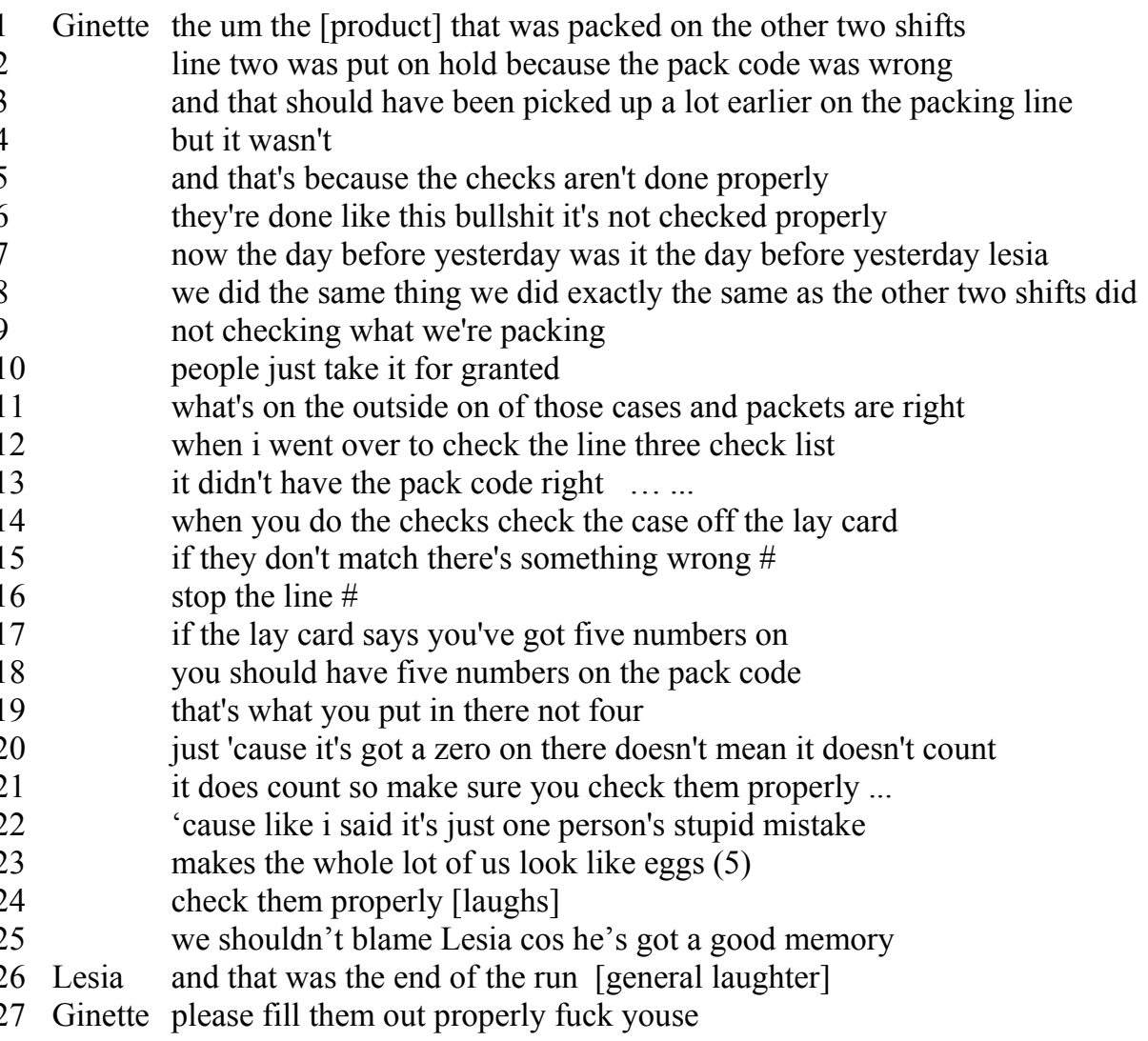


What exactly was the message here? In terms of content, it can be analysed into four main topical themes: (i) there was a recurring problem with the packing codes; (ii) this needed the team's serious attention because it was causing production delays; (iii) it was a tricky issue, but careful checking was the key; (iv) detailed instructions about exactly what was required.

Ginette used a varied array of discourse strategies here to 'get the message across' in all its complexity. She was both direct and explicitly directive in communicating this information, pulling no punches, and dominated the floor for most of this sequence, making heavy use of repetition, lengthy explanations and explicit directives to convey her four main content points. She began by identifying the problem and the reason for it very explicitly: behaviour which causes production delays: line two was put on hold because the pack code was wrong (line 2) and that's because the checks aren't done properly (line 5). Next she gave very clear and detailed instructions about what the packers should do if they see a discrepancy (lines 14-16), using a deliberate, emphatic intonation pattern, when delivering assessments and imperative statements (eg if they don't match there's something wrong; stop the line\# - line 16). She then went on to analyse the problem further and identifying what she surmised to be the source of the problem - a misunderstanding of the significance of a zero in the packing code just cos it's got a zero on there doesn't mean it doesn't count (line 20). Ginette thus moved from an unforgiving analysis of the errors that had been made to a very clear and explicit account of precisely what needed to be done to rectify them, and why.

At the same time she used a number of complementary strategies to convey various layers of affective meaning. Firstly, she made it very clear that she was very annoyed about the situation and critical of the team for their poor performance. Her tone of voice clearly signalled her irritation, as did the degree of repetition and remarks such as that should have been picked up a lot earlier (line 3). She also more subtly reinforced her status as 'the boss' in this instance by indexing her supervisory role and responsibilities in line 11 (when $i$ went over to check the line three check list). There was also a clear inference that the team should be pulling together better - they were all in this together.

Her explicit appeal to the importance of team spirit (one person's stupid mistake makes the whole lot of us look like eggs - line 22-3) along with her use of humour, 
and the tongue in cheek juxtaposition of the very polite please fill them out properly with a strong expletive fuck youse (line 27) all reflected her strong orientation to the team culture and to maintaining team morale. Ginette's use of impersonal constructions and the pronoun we in lines 8 and 10 (we did the same thing; people take it for granted) also indicated she was laying the blame squarely at the door of her team; by insisting that they accept collective responsibility for the mistake, she avoided embarrassing the individuals who were actually at fault (although she did know who they were). However, although she avoided pointing the finger at any individual, in line 25 she quite specifically, though jokingly, let her 'second-incommand' off the hook with we shouldn't blame Lesia cos he's got a good memory (line 23). She concludes with the kind of team-building jocular abuse (line 24) which, as illustrated in chapter 6 , forms the basic currency of the interactions of this team.

This first excerpt in the sequence illustrates how the various transactional and social dimensions are interwoven in Ginette's handling of a serious misunderstanding, and how she was able to move seamlessly between different subject positions. In the space of just a few minutes, she adopted direct and explicit strategies which communicated the problem clearly, and which also instantiated her authority as team leader. At the same time, however, she paid attention to the face needs of team members by avoiding laying blame on any individual, appealing rather to their team loyalty and solidarity. She also made sure she defused the tension at the end of her 'lecture' by raising a laugh and showing that as well as tough talking, she was still the same Ginette who could have a laugh by engaging in the kind of swearing and jocular abuse which were key features of the team's communicative and social culture. These strategies all reflected her strong orientation to maintaining team morale, and her ability to balance her management responsibilities with her continued acceptance as one of the team.

After this briefing, Ginette reported that she was still not confident that the team were really taking the problem seriously, because the individuals she was most concerned about had not appeared to be paying attention during the briefing. However, this perception was not entirely borne out by an analysis of several interactions which took place on the packing line later the same morning. Helena, Lesia and Sam were working near one another, and they had a series of discussions 
as they worked to try and sort out exactly what they needed to do about the packing codes, which were turning out to be a far from straightforward issue. In excerpt (2) we see Lesia trying to show Sam what to do, and using the strategy of invoking Ginette's authority when he resisted Lesia's attempt to correct his mistake.

(2) Problem with the packing codes (b)
Lesia but now they try to take out the zero no more zeros
Sam no 'cause the zero doesn't mean anything the zero is a nothing that there is the main one four five six seven
but the zero zero is only just something in front of it ...
Lesia but why do you think you would say that
when Ginette was explaining that this morning
Sam
oh i wasn't over here
i only just just realised this morning when you come over you see

Lesia began by framing the problem as Ginette had earlier, namely, that people had mistakenly been deleting the zeros in the packing codes (line 1). Sam challenged this, and provided an explicit example of the error that Ginette had earlier identified, by arguing that the zeros had no meaning (lines 2-4). At this point Lesia appealed to Ginette's authority and cited her early morning instructions on the topic, at which point Sam admitted not paying attention during the meeting, and backed down (lines 7-8). This example indicates how easily a problem can be compounded because of a misunderstanding by a single person. If Sam had been more pig-headed or assertive, or if he had been in a position of greater authority than Lesia, it is possible that his mistaken version of events would have won the day. Here the misunderstanding was rectified, but it took more than a simple assertion to convince Sam. A reminder of the morning's briefing which served as an appeal to Ginette's authority was what finally convinced him to (apparently) comply with Lesia's suggestion, and to offer an apologetic justification for his earlier failure to do so.

Excerpt (3) shows that Sam's alignment with Lesia's attempt to correct his error was more apparent than real. In a brief exchange shortly afterwards involving Sam and Helena, a different team member on the packing line, Helena pointed out that Sam was still not copying down the codes that actually appeared on the boxes of product coming off the line as Ginette had instructed the team to do that morning. Sam initially acknowledged this comment with okay (line 4). However after a short while he gave up copying the codes once more, with a complaining shit (line 5). After showing her disapproval with a warning hey hey (line 6) Helena then invoked the 
spectre of being shown up in front of the rest of the team at the next briefing to gain Sam's compliance in an amicable way.

(3) Problem with the packing codes (c)

\begin{tabular}{|c|c|}
\hline 1 Helena & so you can't copy that you gotta copy what you got in the box \\
\hline 2 & 'cause you get your box off the line right so everything in the box \\
\hline 3 & is what you're going to write down there \\
\hline $4 \mathrm{Sam}$ & okay (11) - n \\
\hline 5 & shit \\
\hline 6 Helena & hey hey next week next week at er the team brief \\
\hline 7 & me and you are gonna get singled out \\
\hline 8 & next time you're told to do something do it \\
\hline 9 Sam & good on ya good on ya Helena \\
\hline
\end{tabular}

This time the strategy used was an explicit appeal to the team ethic (lines 6-7). This indirectly referenced Ginette's comment at the end of the briefing that if individuals did not make an effort to sort this problem out, then they would be letting the whole team down. Helena elaborated this with the threat of me and you being singled out at the next team brief, which warranted the very explicit directive which followed: next time you're told to do something do it (line 8). At this point, Sam's resistance finally appeared to crumble, with his resigned sounding closing comment good on ya Helena.

These excerpts have provided a taste of how the messages that Ginette was trying to convey during the briefing continued to be negotiated by others in the team throughout the morning. Lesia and Helena at least had understood that there was a problem that they needed to take seriously; even though Sam had clearly not understood all the details of what was needed, his co-workers were actively working on resolving the issue in a collaborative fashion during their successive interactions with him. The affective messages that we saw in Ginette's monologue were also taken up, and were being renegotiated in various ways, especially the notion that if individuals did not make an effort to sort this problem out, then they were letting the whole team down. Ginette's mana (standing) as team leader along with the strong team ethic were powerful strategies for gaining compliance, which Ginette had made especially salient by the way she had delivered her 'message' earlier in the shift.

Finally, in excerpt 4, we see Ginette in action again, this time talking one-to-one with Sam during her 'round' of the packing line towards the end of the morning. Sam was clearly still a little confused, but now in a much more cooperative frame of mind. 
Again, what is noteworthy is Ginette's skill in combining clarity of explanation with attention to the affective dimensions of the interaction.

(4) Problem with the packing codes (d)

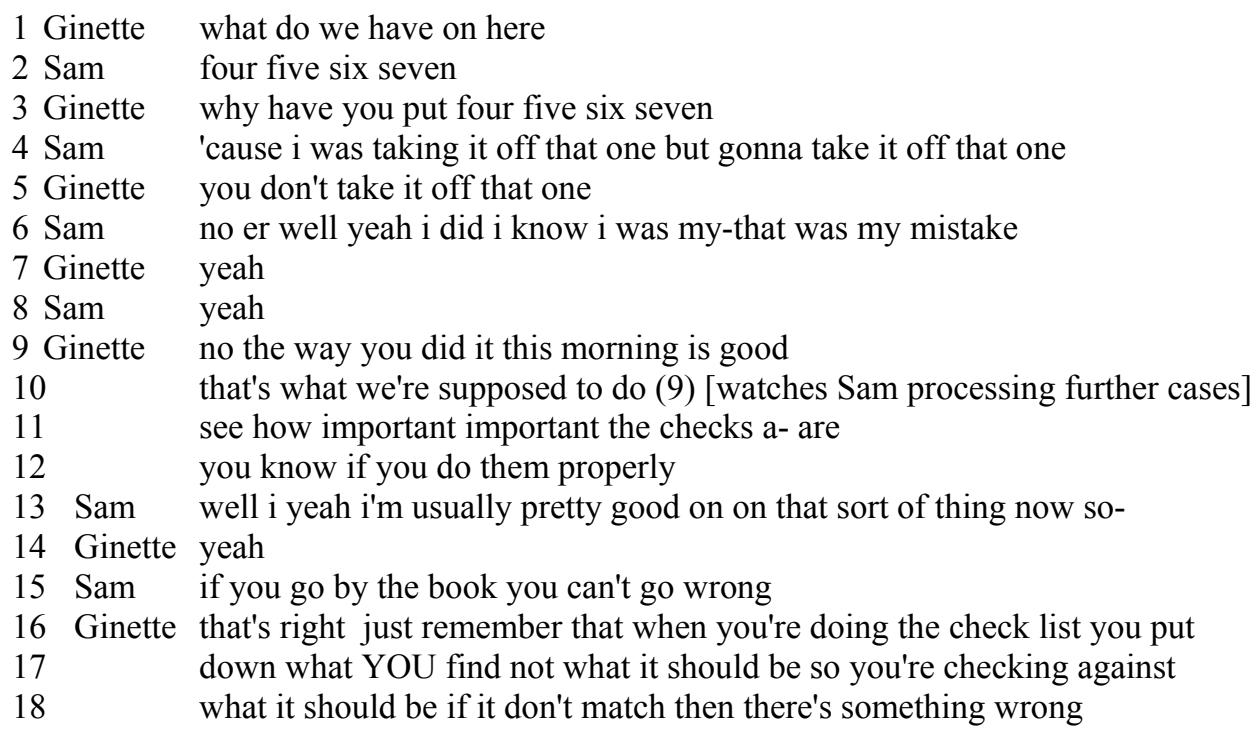

What is interesting here is the way in which Ginette adopted quite different strategies to those she used when she was talking to the whole team. In this setting she used a range of facilitative, 'coaching' strategies to help Sam see for himself what he had been doing wrong (line 6) and what was right (line 9), and by the end of the interaction she had got him to the point where he acknowledged how important it is to pay attention to detail and do the checks (line 15). She asked questions (lines 1 and 3), corrected a misunderstanding - you don't take it off that one (line 5), provided supportive feedback yeah (lines 7 and 14) and positive reinforcement of what Sam was doing well (line 9-12), echoed and expanded on Sam's contributions, and then finally summed up what they had agreed (lines 16-18).

It took all morning, but at least in relation to this worker, the problem had finally been resolved. A key analytic point here is that a final resolution of the misunderstanding was not achieved in a single interaction. It took a series of related interactions in different settings with different interlocutors, skilful balancing of a range of direct and indirect strategies, and invoking of affective as well as referential meaning, for the message to finally "get across", as Ginette put it. Few misunderstandings are so clearly identified and explicated in any case, but it would have been impossible to uncover these complexities without tracing the negotiation of the problem through a 
series of interactions between different team members throughout the day following the morning briefing session.

The ethnographic field data also provided important contextual information about the TCO's analysis of the practical significance of the problem, the 'back story' of how it had arisen, and which team members were most likely to be generating it. This not only flagged the issue as one worthy of analysis, but also made it possible to record key interactions and thus track and analyse in some detail how this issue unfolded from the time Ginette first articulated it publicly, and to follow the progress of her and other team members' attempts to rectify it. As a result, it was possible to directly observe and analyse the varied strategies that were used to resolve the problem, ranging from very explicit and direct denunciations of incorrect behaviour, and appeals to authority to explicit coaching and invoking of team solidarity to ensure the problem did not recur.

The analysis of this 'critical incident' was further enriched by the insights already gained into the team's communicative culture and the TCO's observed practices and ethos, by having previously undertaken a 'thick description' within a community of practice framework. Next we turn to a more explicit consideration of a different kind of problematic talk, namely the interactional dilemmas that arose in relation to 'doing' power and solidarity as Ginette managed the contradictions between the different roles and identities she enacted within the team.

\subsection{Applying a critical lens}

\subsubsection{Power, identity and miscommunication}

From a critical perspective, power is always relevant to any analysis of the way people interact and discursively construct their social identities and relationships. ${ }^{2}$ The dimensions of power, solidarity and identity are especially pertinent to the analysis in this chapter of the strategies used by Ginette to manage miscommunication and problematic talk, because of the tensions and contradictions inherent in her dual roles as TCO and team member. Her cross-cutting membership

\footnotetext{
${ }^{2}$ See also the more detailed discussion of this and related points in chapters 2 and 3.
} 
of a range of other social and occupational groups within the factory adds a further layer of complexity to the analysis.

As we have already seen, from Ginette's perspective, facilitating effective communication and maintaining good relationships (within the team, between herself and the team, and with the rest of the organisation) were essential to achieving her goals as a team leader. She herself was a highly skilled communicator with a wide discursive and linguistic repertoire at her disposal for dealing with problematic issues as they arose, and for helping to prevent problems from arising in the first place. Ginette was popular and well-respected by her co-workers, and her communicative competency and flexibility contributed in no small measure to the Power Rangers' performance as an effective and highly-motivated production team with a strong ethos of camaraderie and collective responsibility.

However, a critical analysis requires us deconstruct this assessment further to ask whose interests were being served in this case, and to what extent those interests were in conflict or alignment with one another. In terms of the core business of the organisation, the most important consideration was Ginette's ability to influence the behaviour of the team in order to achieve the managerial goals of maximising productivity and safety, and therefore company profits. Clearly, the management had a vested interest in the team performing as well as possible for the benefit of the factory shareholders, and anyone in a middle management role had a clear responsibility for delivering on this as a bottom line. Inevitably, positive outcomes would also add to Ginette's personal mana and identity as a capable (female, Polynesian) manager, and to her own career progression prospects. ${ }^{3}$

From both of these perspectives, Ginette's skilful facilitation of and participation in the positive team culture of the Power Rangers could be analysed as an example of what Fairclough (1992b) terms "repressive discourse". This refers to the use of discourse strategies by those in positions of authority which covertly or indirectly help gain the willing cooperation of those in relatively powerless positions, rather than via the direct wielding of power. This may well be at least partly the case here. However, even if so, this does not rule out the possibility that Ginette is acting simultaneously, or instead, in an empowering way. That is, in the (perceived) best

\footnotetext{
${ }^{3}$ Two years after the completion of the study, Ginette was in fact 'headhunted' for an operational management position in a larger plant belonging to the same corporation.
} 
interests of the people in her team within the microcosm of the factory, even though the underlying wider social and economic inequalities remain firmly in place.

That is certainly how Ginette herself reconciled the tension between these two positions, describing her approach as delivering a 'win-win result' which was of mutual benefit to all parties. Workers need their jobs too, and their excellent performance meant that this team and its individual members benefited materially (e.g. bonuses, training and overtime opportunities, possible career progression), as well as in terms of intangibles such as job satisfaction and self-esteem, especially by comparison with other less cohesive and successful teams. There were also additional benefits like a sense of belonging, positive relationships, camaraderie, and a more interesting and stimulating work environment (by factory production line standards anyway). An alternative way of characterising the power and solidarity dimensions of Ginette's role then might therefore be as a mediator or power broker.

Although such issues may seem at first sight to be of mainly theoretical interest, there are in fact several ways in which the enactment of power may cause or interact with miscommunication to create a risk of more immediate practical consequences. Firstly, overt or direct attempts to enact or contest power at a localised level of interaction often run the risk of leading to miscommunication, especially at a relational level, because of the potentially face-threatening nature of such formulations (though as we saw in chapter 6, there is not always a straightforward correlation). Conversely, using more indirect discourse strategies to 'save face' (or to be linguistically polite) rather than enacting power explicitly, can also lead to cases of localised misunderstanding or more extended miscommunication of either a practical or relational nature.

The relationship can flow in the other direction too. Thus, as discussed in previous chapters, actual miscommunication or non-communication (or attributing a problem to these), may be used as a strategic resource, either for 'doing' or resisting power at the local level of the interaction, or for 'playing power games' that are embedded in wider institutional or even societal discourses. Finally, at a more global level, if miscommunication and problematic talk become too frequent or are not wellmanaged within a team, this can potentially escalate to create ongoing power struggles or conflicts. Conversely, if power relations become overly coercive or contestive, this may in turn create negative dynamics which make localised 
miscommunication more likely to occur, and less easy to repair. Either situation would constrain the TCO's ability to effectively exert relational power and thus influence behaviour to achieve her managerial goals.

From a practical perspective, therefore, miscommunication and conflict are key risks the TCO has to manage, and managing the team effectively by definition requires proactively managing communication, miscommunication and power relations.

Table 7.1: Types of power relationship

\begin{tabular}{|c|c|c|}
\hline \multicolumn{2}{|c|}{ Type of Power } & Power based on: \\
\hline \multirow{6}{*}{$\begin{array}{l}\text { Control } \\
\text { "POWER } \\
\text { OVER" }\end{array}$} & Legitimate & $\begin{array}{l}\text { Formal status (a person's role or position within an } \\
\text { organisation) and associated rights } \\
\rightarrow \text { Exert authority }\end{array}$ \\
\hline & Coercive & $\begin{array}{l}\text { Use of force ( emotional or physical) in the face of } \\
\text { resistance } \\
\rightarrow \text { Gain compliance }\end{array}$ \\
\hline & Expertise & $\begin{array}{l}\text { Specific skills, abilities or knowledge held by an } \\
\text { individual or group } \\
\rightarrow \text { Provide assistance }\end{array}$ \\
\hline & Reward & $\begin{array}{l}\text { Control over desirable resources or 'goods' } \\
\text { (including relational 'gifts' and 'permissions') } \\
\rightarrow \text { Grant access }\end{array}$ \\
\hline & Personal & $\begin{array}{l}\text { Individual mana, personality or charisma (role } \\
\text { models) } \\
\rightarrow \text { Exert influence }\end{array}$ \\
\hline & Social & $\begin{array}{l}\text { Social status and associated rights (derived from a } \\
\text { person's role or position within a group or society) } \\
\rightarrow \text { Exert influence and/or authority }\end{array}$ \\
\hline \multirow{2}{*}{$\begin{array}{l}\text { Consent } \\
\text { "POWER } \\
\text { TO" }\end{array}$} & Collaboration & $\begin{array}{l}\text { Joint influence, negotiation or consensual decision } \\
\text { making or action } \\
\rightarrow \text { Offer mutual engagement }\end{array}$ \\
\hline & Empowerment & $\begin{array}{l}\text { Facilitation or genuine 'giving up' of power to } \\
\text { others who are relatively power-less } \\
\rightarrow \text { Delegate or share power }\end{array}$ \\
\hline
\end{tabular}


For the analysis of Ginette's discursive repertoire to follow, it will be useful to deconstruct the concept of power itself a little further. The theoretical justification for classifying power into different types was explained in chapter 2 (see 2.3.5). The taxonomy set out above in Table 7.1 is my own synthesis of the models of power proposed by Pateman (1980), Fairclough (1989; 1992b); Dwyer (1990) and Ng and Bradac (1993), adapted and somewhat simplified to suit the more limited purposes of the analysis here. These categories are not mutually exclusive, and are not proposed as invariant characteristics of any particular person or role; rather, they are intended to provide a broad conceptual framework for analysing how power relationships are dynamically co-constructed in a given interaction.

These different types of power relationship may simultaneously arise from, and function as a resource for, the co-construction of interpersonal and/or intergroup identities in interaction. Critical discourse analysts would argue that power is an omnipresent dimension of any interaction. However, the current analysis has been limited to examples where there is evidence in the discourse that the negotiation of power and/or identity was oriented to by the participants as being in some way problematic, or where these constructs were themselves used as an interactional resource to manage some other problematic aspect of the interaction.

\subsubsection{Managing the contradictions}

Within a critical social constructionist framework, discourse is seen as a dynamic set of strategies whereby people are continually (re)constructing various overlapping personal, ethnic, gender and professional identities (amongst many others). As discussed in chapter 2, identity is not regarded as a single static characteristic in this paradigm, but instead is viewed as an ongoing process of social construction which takes place in and through discourse. Within a community of practice, the shared knowledge and practices of the group are available as resources for this process of identity construction, along with the personal discursive repertoire of the individual, and the identities produced in this way themselves become available for use as interactional resources in their turn.

In her role as TCO, Ginette faced particular challenges in finding a balance between frequently contradictory sets of identities and goals. In order to retain her mana as a leader she had to meet her responsibilities to the factory management, making sure 
the work got done on time and to the right standard, while at the same time having a substantial personal investment in looking after the particular interests of her own team. She had assessed the development of team morale and unity as being a key function of her role as TCO, and, as we have seen, within the culture of this particular team, that required being accepted as 'one of the crew' and standing as 'first amongst equals', something she also identified with and aspired to at a personal level.

Like the other team members, Ginette actively participated in the prevailing culture of joking and teasing, and in fact often exploited her dual identities as team leader and team member by deliberately initiating such activities as a way of countering boredom and maintaining morale. However, given the nature of the working environment and the diversity of skills and backgrounds within the team, it was often also necessary for her to explicitly take charge and 'lead from the front', as shown in the packing codes incident in the previous section.

Further complicating Ginette's situation was the fact that she was a relatively young New Zealand Samoan woman with a leadership role in a cultural and work context where women and members of minority ethnic groups have not traditionally been placed in formal positions of authority. As a result, there were numerous expectations of her (often conflicting, some of them self-imposed) - as a leader, as a representative of or advocate for different groups, or just as a capable individual.

The factory management encouraged her to act as a role model for other team leaders and staff in the factory, and made extensive use of her skills and leadership abilities over and above her principal role with the Power Rangers. Women, in her own team especially, looked to her for 'moral support' in the male-dominated environment of the factory. The Polynesian workers, in particular the Samoans, also gravitated to her as an important source of support and mentoring. In addition, Ginette took it upon herself to similarly nurture the individual and collective development of her own team, regardless of gender or ethnicity.

The interactional examples below demonstrate that not only did Ginette 'manage the contradictions' between her different roles and identities (or aspects thereof) extremely skilfully, but she also turned them to strategic advantage with respect to achieving her interactional and managerial goals. Her ability to switch between 
and/or index a number of different identities or inter-subjective positions in her interactions is a key to understanding how she was able to manage potential miscommunication and conflict so expertly. In this final part of the case study, we turn to a critical analysis of how Ginette made strategic use of an impressively wide discursive repertoire to skilfully manage the contradictions between different roles or identities that were seen to be typically enacted in her interactions with her teammates. These can be grouped into six broad identity positions which are primarily associated with certain types of power, as summarised in Table 7.1.

Table 7.2: Identity positions constructed by the TCO

\begin{tabular}{|c|c|c|}
\hline Identity positions: & \multicolumn{2}{|c|}{ Primarily associated with: } \\
\hline $\begin{array}{l}\text { 1. The boss: } \\
\text { 'in charge and on-task' }\end{array}$ & $\begin{array}{l}\text { Legitimate power } \\
\text { Coercive power } \\
\text { Reward power }\end{array}$ & $\begin{array}{l}\text { Exerting authority } \\
\text { Gaining compliance } \\
\text { Granting access }\end{array}$ \\
\hline $\begin{array}{l}\text { 2. The coach: } \\
\text { 'guiding and mentoring' }\end{array}$ & $\begin{array}{l}\text { Expertise power } \\
\text { Empowerment } \\
\text { Legitimate power }\end{array}$ & $\begin{array}{l}\text { Providing assistance } \\
\text { Facilitating } \\
\text { Exerting authority }\end{array}$ \\
\hline $\begin{array}{l}\text { 3. The advocate or mediator: } \\
\text { 'go-between' }\end{array}$ & $\begin{array}{c}\text { Personal power } \\
\text { Collaboration } \\
\text { Legitimate power }\end{array}$ & $\begin{array}{l}\text { Joint influence } \\
\text { Negotiation } \\
\text { Indexing authority }\end{array}$ \\
\hline $\begin{array}{l}\text { 4. The matriarch: } \\
\text { 'camp mother' }\end{array}$ & $\begin{array}{l}\text { Social power } \\
\text { Personal power } \\
\text { Reward power }\end{array}$ & $\begin{array}{l}\text { Exerting authority } \\
\text { Exerting influence } \\
\text { Granting access }\end{array}$ \\
\hline $\begin{array}{l}\text { 5. The team member: } \\
\text { 'one of the crew' }\end{array}$ & $\begin{array}{c}\text { Collaboration } \\
\text { Personal power } \\
\text { Expertise power }\end{array}$ & $\begin{array}{l}\text { Mutual engagement } \\
\text { Exerting influence } \\
\text { Providing assistance }\end{array}$ \\
\hline $\begin{array}{l}\text { 6. The informal leader: } \\
\text { 'first amongst equals' }\end{array}$ & $\begin{array}{l}\text { Empowerment } \\
\text { Collaboration } \\
\text { Personal power }\end{array}$ & $\begin{array}{l}\text { Delegating, sharing power } \\
\text { Mutual engagement } \\
\text { Exerting influence }\end{array}$ \\
\hline
\end{tabular}

The examples that follow are loosely grouped according to these identity position(s) as observably oriented to by the participants in each interaction.

The boss: 'in charge and on-task'

An analysis of the recorded data set as a whole confirmed the ethnographic observation that when giving instructions or meting out criticism, Ginette often adopted a plain speaking, very direct, sometimes almost authoritarian style, particularly when dealing with the team as a group. This style seemed to be 
associated most often with the first identity position listed in the table above, namely that of 'the boss' who is assertively 'in charge' and 'on task', an identity position that was warranted in the first instance by the legitimate power arising from her role as team coordinator.

Example (5) is a straightforward example of this with Ginette conveying routine information and instructions to the team at one of the morning briefings. There is no evidence here of the humour and banter typical of the Power Rangers style of interaction in other contexts. This set of instructions was delivered in a terse public announcement style marked by strategic repetition of key information (marked in bold). Note the way the end of each instruction is signalled by a pause of up to a second, and the way in which Ginette neatly accepts and incorporates the correction to her terminology offered by one of the men (calcite for her incorrect fulcite) without breaking the stride of her delivery.

(5) Bagging off

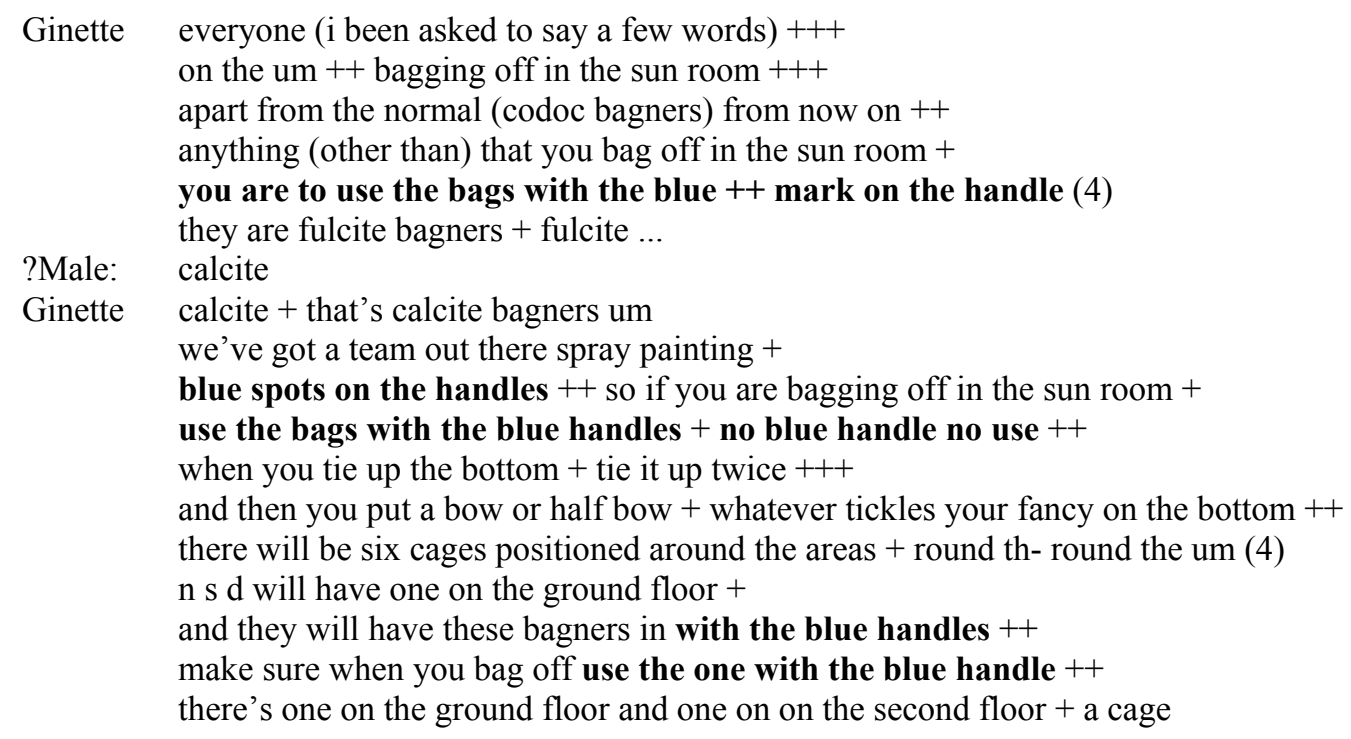

Example (6) is another example of a superficially very matter of fact delivery of information as part of Ginette's legitimate exercising of her power as team coordinator to enforce certain administrative requirements. There was arguably an indirect element of coercive power here too, as seen in the reminder that management (currently represented by Ginette) would withhold sick pay if the relevant leave forms continued not to be submitted. At the same time, the way the instruction is formulated (by way of a neutral observation I noticed ... and a factual description in the passive voice before you get paid...) distances Ginette from the administrative process. 
Although the 'enforcement' message is clear, there is also an element of coaching, a more empowering identity position associated with facilitation and assistance based on superior knowledge or expertise power rather than the simple exerting of authority based on legitimate power.

(6) Sick leave

G: $\quad$ I noticed that people haven't been putting in sick leave forms +

M: what's that

G: before you get paid ++ you still have to fill out a sick leave form

M's clarification request what's that draws attention to the functional ambiguity of Ginette's remark, requiring her to make explicit the connection between submitting the forms and getting paid. However, there is no 'right' interpretation here, and that is exactly the point; rather, Ginette was using very subtle devices to simultaneously index two or more possible identity positions, leaving the team in no doubt of the need to obey the rules, but nevertheless framing this in a non-threatening facilitative way.

As we see in the next excerpt, the persona of 'the boss' was not infrequently instantiated in a stereotypically very 'unfeminine' way of talking, reflected in the liberal use in such contexts of unmitigated negative or overtly face-threatening speech acts such as explicit directives, criticism, telling off, complaining or correction, along with the free use of expletives on many occasions that we have seen in previous examples.

(7) Stacking boxes

Ginette when yous take them off stack them properly

David oh //so who was $\backslash$ taking them off from here and loading it

Russell /it was [name]\\} I always stack back on

Sam you're assuming eh assuming bullshit eh

Ginette no I said what did I say + I said when you take them off stack them properly that wasn't an a- assumption // that was a- I

Sam: //stacking it properly /you assumed that $\backslash \backslash$ somebody was not

Ginette / that was an $\backslash \backslash$ instruction

Sam: you were assuming that someone has not taken it off but not ( )

Ginette so if I come and they're in a big heap

Sam: what

Ginette they're stacked properly?

Sam if you what

Ginette if I come in and they're all on the wrong way on the pallet does that mean they're stacked properly

Sam beautiful

Ginette bloody [drawls] :beautiful: 
Here Ginette was giving Russell, Sam and David instruction on how to stack boxes correctly. Sam was being argumentative, and Ginette's restatement no ... what did I say... and her assertion that wasn't an assumption ... that was an instruction both served to index her legitimate power to tell team members what to do. An annoyed tone of voice towards the end of the exchange also indicated that Ginette interpreted Sam's retorts and interruptions as contesting her authority rather than as anstance of jovial banter on this occasion.

There was possibly also a flavour of coercive power being invoked here, implicit in Ginette's persistence in her attempt to gain compliance in the face of Sam's continued resistance, but her ironic echoing of Sam's concession (beautiful) that she was in fact right, softened the effect of her previous insistence and allowed the interaction to end on a positive note. Excerpt 2(a) above, where Ginette was taking the team to task at the morning briefing over their errors with the packing codes, was another typical example of this pattern. As we also saw in that excerpt, Ginette's 'straight shooting' style was more often than not leavened with humour.

She thus typically tempered and blended this direct 'do what I say, or else' style with a range of both collaborative and contestive discourse strategies which served overall to mitigate the directness of her delivery, ending with a humorous retort to defuse tension. This balancing act allowed her to continue to build team solidarity and minimise the effect of the difference in status between team and manager whilst at the same time getting the transactional 'message' across unambiguously.

Ginette's direct style in these interactions worked well, partly because it accommodated to the stereotypically masculine discourse norms of the factory, but more importantly because she had developed a strong, positive relationship with her team. They trusted her and were confident that she would look after their interests when dealing with higher management, for instance. They also knew very well that later in the day she would be just as ready to join in with a joke or a tease as anyone else in the team and that she did not abuse her position of authority. This shared understanding and experience thus constituted another interactional resource that could be drawn on in the co-construction and interpretation of such sequences, as will be further illustrated via the next example. 


\section{The coach: 'guiding and mentoring'}

Dealing with team members who needed help on a one-to-one basis or in training sessions, Ginette's style was often quite different. She was sensitive to their particular problems, and took care to preserve their dignity. In such situations, whilst still very clearly 'in charge and on task', Ginette acted more as a coach or mentor, following up on what the team member was doing, leading them through the solution to a problem and patiently waiting and encouraging them to work out things for themselves rather than simply demonstrating or instructing. Although predicated on a base of legitimate authority, when constructing this identity position Ginette appeared to draw primarily on expertise power and empowerment, where the aim was to pass on knowledge and skills to allow the worker concerned to operate as autonomously as possible.

In the packing codes incident, we saw an example of this in the way Ginette took Sam through the process one more time, gently affirming his newly achieved understanding and more positive attitude towards the task, in an approach which contrasted starkly with her rather abrasive briefing on the matter earlier in the day. In example (8), Ginette was training one of the packers, David, who had literacy issues, on the use of ticket numbers on the packaging.

(8) Ticket numbers

\begin{tabular}{|c|c|}
\hline David & $\begin{array}{l}\text { o- one thing i'm not }+ \text { totally clear on } \mathrm{i} \text { can see my own ones here so i'll talk about } \\
\text { them }+ \text { up in up in the top box it's got the pack code stroke material number }\end{array}$ \\
\hline Ginette & $\mathrm{mhm}$ \\
\hline David & $\begin{array}{l}\text { i've }+ \text { not always but i fairly often put the ticket numbers in there }+ \text { now what's the } \\
\text { story about that because i've //got } \mid\end{array}$ \\
\hline Ginette & $/$ the $\backslash \backslash$ pack code there's two these sheets here are used \\
\hline & for $/ /$ two reasons $\backslash$ \\
\hline David & /two purposes $\backslash \backslash$ yeah (it's got) \\
\hline Ginette & $\begin{array}{l}\text { (yeah) it's pack stock down here now we're using them for (product) and also raw } \\
\text { materials }\end{array}$ \\
\hline David & yep \\
\hline Ginette & so when you're dealing with the the stock down here it's your pack code \\
\hline David & yep \\
\hline Ginette & and the material number is for the raw materials \\
\hline David & yep okay \\
\hline Ginette & $\begin{array}{l}\text { yeah }+ \text { but you wouldn't necessarily need that for }+ \text { um bagging off and all that kind } \\
\text { of stuff unless it's s- stock that you're cutting open into a bag }\end{array}$ \\
\hline David & yep \\
\hline Ginette & yeah? does that answer your question David \\
\hline David & $\begin{array}{l}\text { yep because sometimes it's c- can build up to like eight or nine hundred kilograms } \\
\text { on the one piece of paper so i've used that .... }+ \text { and then i've thought well shit }+ \\
\text { now it you know obviously they know that's it's not gonna be nine hundred } \mathrm{k} \text { in a } \\
\text { bag so i'd better put down the ticket numbers }\end{array}$ \\
\hline
\end{tabular}


Notable here was the sensitive manner in which Ginette responded to David's question, which actually related to a very basic routine matter. This was reflected in the time she took to hear David out with his questions and interpretations, her careful and patient explanation of the things he was struggling with, and her explicit checking that he now understood (yeah? does that answer your question David).

Ginette's ability to switch in this way to a quite different register was an important component of building and maintaining trust. On the basis of his past experience of Ginette's management style, David clearly felt it was safe to ask any question, no matter how trivial, and admit to his 'boss' that he had handled the matter incorrectly in the past (I fairly often put the ticket numbers in there + now what's the story about that) without fear of being met with either criticism or impatience. The 'coach' identity therefore represented an important discursive resource for building resilience and safety netting into the team's communication practices.

\section{The advocate or mediator: 'go-between'}

This next example shows Ginette wearing the hat of a mediator by acting as gobetween to help resolve a particular incident. The identity positions of 'matriarch' and 'informal leader' also comes into play here. This interaction deals with one part of an ongoing problem relating to the working relationship of one of the fitters, Rick, with a number of other workers on the packing line. The fitters' job was to maintain the machinery, and troubleshoot any mechanical or technical problems which occurred. Rick was a Pakeha in his mid-twenties who had begun with the company as a young apprentice. The incident referred to in this example involved Helena, a Maori woman in her mid-thirties, and a very experienced team member. Helena had in fact shared the TCO position with Ginette until a year previously, when it was decided by the team that it would be more efficient to have just one person in this role, so the two had a close working relationship. The excerpt below comes from a conversation between Ginette and Helena which was part of a quite complex sequence of events, so in order to understand what is going on in this particular interaction, it will be necessary to first provide some background detail.

From the ethnographic data we know that Ginette felt Rick's attitude had been an underlying problem for some time. As reflected in the excerpt, she was concerned that he didn't always focus on the work at hand, and didn't recognise the need for 
urgency in responding to calls - his priorities often did not seem to be aligned with those of the rest of the team. Other team members had also been complaining to her, and she had heard them 'whingeing' to one another about his attitude and unreliability, which made their work go less smoothly. She had also noticed that Rick did not seem to get on very well with Simon, the other fitter, whose work habits were more compatible with the collaborative way the rest of the team liked to work and interact. Simon had come to the job from a different trades background, so Rick considered him to be less competent, and there was often minor conflict between them.

At the time of the conversation between Ginette and Helena below, there had been a rash of recent problems with the conveyor belts on the packing lines. Cartons were being ejected even when they were not faulty, there had been a recurring problem with a sensor not working properly on the checkweighing machine, and the 'Windsor', a machine which stacked the cases of soap powder cartons at the end of the conveyor belt, kept jamming halfway through a run, which meant that packers had to stack the cases by hand, and sometimes had to stay late to complete a run. Helena had spoken to Ginette earlier in the day about these problems and her annoyance with Rick's attitude. She was generally quite assertive about dealing with interpersonal issues herself, but felt she was not succeeding in this case, as the problems with Rick kept recurring, and were starting to have serious consequences.

The particular incident which triggered her complaint had occurred that morning. She had called Rick over to have a look at the problems with the checkweigher and the Windsor, but he responded that the machine operators should be able to fix it themselves, and had refused to come when asked. In fact, it did prove to be a problem that was a fitter's responsibility, and because Rick did not attend to it when Helena first asked him to, there was a 40 minute stoppage on the affected packing line, which meant the team fell seriously behind with their production targets.

Later that morning, after Helena's complaint, Ginette spoke to Rick. In the interaction below, Ginette had come to report back to Helena while she was working on the packing line. She told Helena that she had spoken with Rick about his lack of urgency in responding to the packing staff's calls and doing the necessary repairs (lines 1-5). She reported what had happened when she talked to him (lines 9-18), and Helena in turn recounted how a similar thing had in fact occurred again that morning 
just before Ginette talked to Rick (lines 19-26). The interaction between Ginette and Helena is shown below.

(9) Fitters dispute

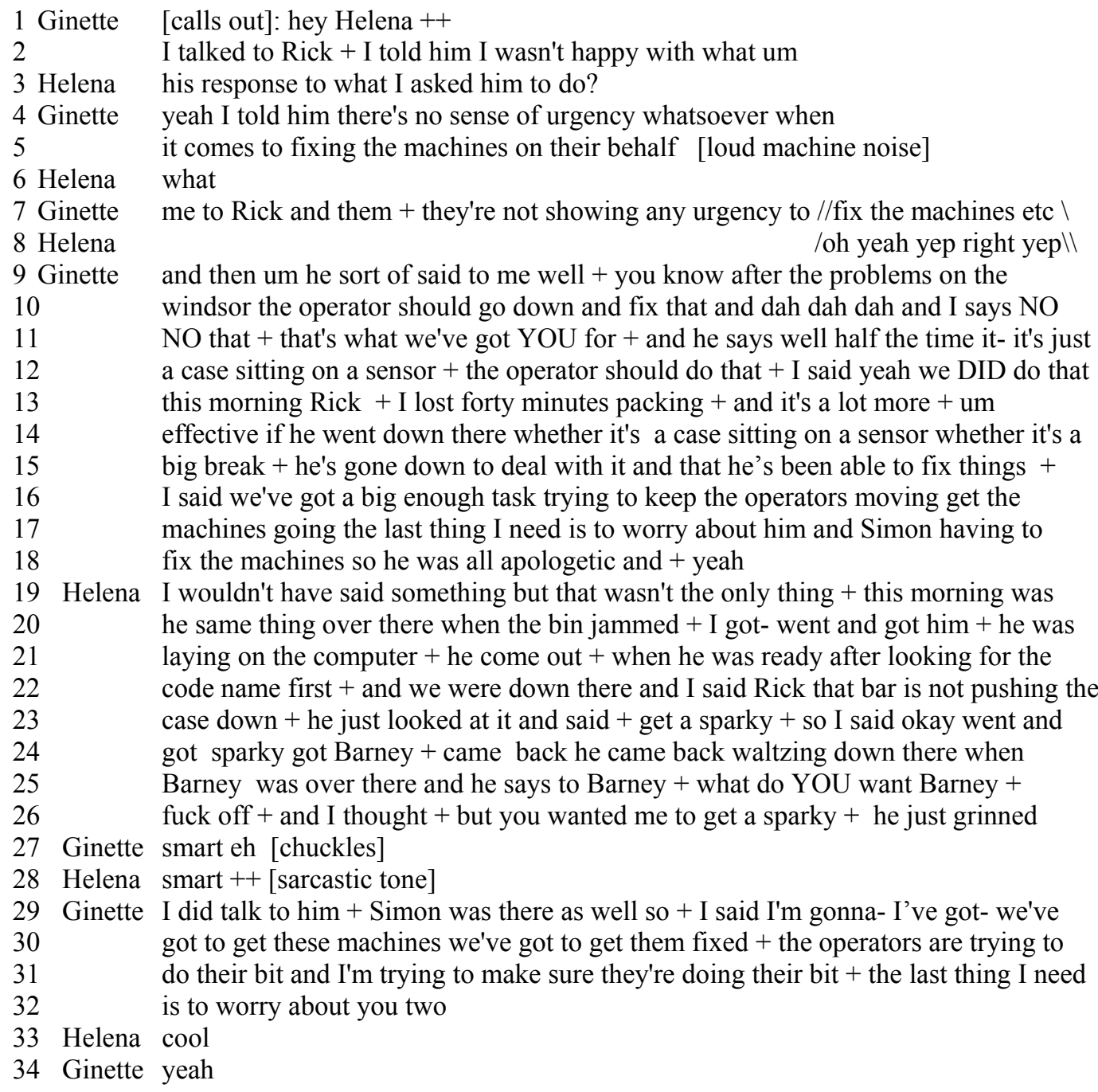

The tone of this interaction is very collegial, and in fact has some structural similarities to a typical conversational 'troubles telling' (Jefferson 1988) with Helena's 'second story' (lines 19-26) following on from Ginette's narrative about her own encounter with Rick. Helena's response I wouldn't have said something but that wasn't the only thing (line 19) served to justify the seriousness of her complaint in the face of Rick's reported apology, and perhaps to cast some doubt on its sincerity. Ginette displayed her solidarity with a sardonic response smart eh, possibly also an attempt to defuse Helena's annoyance. This was echoed by Helena, with Ginette then providing final reassurance that she had indeed given Rick a good 'talking to' and had made her expectations quite clear to both him and Simon, the other fitter. 
This was a very tricky interaction for Ginette to manage at both an interpersonal and a discursive level, and there was some quick footwork involved in moving between and indexing different identity positions and power relationships here. The role of mediator or go-between is itself somewhat ambiguous in terms of power. It may involve advocacy (exerting influence) on someone else's behalf, which is usually based on personal power or mana (and in some cases also on expertise), and/or legitimate power. At other times, it may simply involve the neutral conveying of a message, or facilitating a more collaborative negotiation between the parties. In this case, the lines were not at all clear and crossed over the boundaries of this single interaction.

At one level, Ginette was collaboratively engaging with Helena as a fellow team member in troubleshooting a problematic incident, and this dynamic was very evident in the way their interaction unfolded, as already discussed. It could also be argued that these two women were co-constructing elements of a 'matriarchal' identity as well. The clearest evidence of this in the discourse itself was the shared negative evaluation in lines 27-28 by Ginette (smart eh) and Helena (smart) of Rick's disrespectful treatment of Helena earlier, which placed them both on the same footing. This interpretation is supported by the ethnographic data which tells us that Ginette and Helena used to be co-leaders of the team, both having worked there for a number of years. They shared a strong belief in the importance of collective responsibility within the team, and in their personal lives they were also both mothers of a similar age. Indeed, elsewhere in the data, it is possible to see Ginette explicitly promoting the model of the team as a family that sticks together. This view appeared to be shared by the rest of the team, as shown by the affectionate nicknames 'Camp Leader' and 'Camp Mother' which were used to refer to Ginette and Helena respectively when they were co-team leaders, and still occasionally cropped up in the data collected for this study. ${ }^{4}$

However, at the same time, Helena was also calling on Ginette to mobilise her personal influence and her authority as team coordinator to back her up because she herself had not been able to resolve the issue, and these aspects of Ginette's identity were clearly reflected in the content of her (self-reported) exchange with Rick. This

\footnotetext{
${ }^{4}$ These nicknames are drawn from an act in the repertoire of the popular New Zealand comedy duo the Topp Twins, who portray two bossy female youth camp leaders.
} 
created something of an interactional dilemma for Ginette. Namely, in order to reassure Helena that she had indeed done her best to resolve the issue, and that she was taking it very seriously, she needed to recount in some detail exactly how assertively she had intervened on Helena's behalf, which also meant foregrounding the current difference in formal status between them (e.g. lines 10-11, I says NO NO ... that's what we've got YOU for). Moreover, by demonstrating her own apparent success where Helena had failed (line 18, so he was all apologetic), she was inevitably also demonstrating that her personal influence and ability to influence Rick were greater than Helena's. These were both potential threats to Helena's face and to the solidarity between them, so it required a delicate interactional balancing act to maintain the overall frame of collaborative power and solidarity.

The almost verbatim narrative reporting style of this conversation may have been one strategy on Ginette's part for resolving this interactional dilemma, as it serves to distance her from any direct evaluation of her own success in achieving the desired outcome. The style of the exchange with Rick as narrated by Ginette also plays down her direct exerting of authority as she enacts the role of mediator between the packers and fitters. Her reported criticisms of Rick are formulated as personalised appeals rather than direct instructions (e.g. line 2, I talked to Rick + I told him I wasn't happy; line 13 I lost forty minutes packing; line 31 I'm trying to make sure (the operators) are doing their bit). Ginette also shifts between different identity positions which help reinforce her alignment with Helena. In lines 12-13 she positions herself as 'one of the crew' (I said yeah we DID do that this morning Rick), here taking the side of the packers', and also Helena's by implication. Elsewhere, Ginette's account hints at the exasperated 'matriarch' dealing with unnecessary discord within the family, whilst at the same time indexing her identity as the 'informal team leader' simply trying to get the job done (e.g. line 16-17 I said we've got a big enough task trying to keep the operators moving get the machines going ... the last thing I need is to worry about him and Simon; line 29-32 we've got to get these machines ... fixed + the operators are trying to do their bit and ... the last thing I need is to worry about you two).

The team member: 'one of the crew'

There were many instances where, in interactional terms at least, Ginette appeared to more or less completely cast aside her status as team leader, focusing instead on her 
role and identity as an ordinary team member, who was just 'one of the crew'. This temporarily freed her from the kinds of interactional dilemmas we saw operating in the problem-solving discussion with Helena above, and allowed her to participate in an unconstrained manner in the banter, practical jokes, gossip and whinges which were so important in constructing and maintaining a sense of solidarity and engagement within the team. This willingness to 'have a laugh' also added hugely to Ginette's personal popularity and reputation as a 'good sport', which seemed to provide a kind of global mitigation or 'social insurance' for the times when she had to step in and be very forceful and direct in her management role in order to rectify a problem or to get something done quickly.

Example (10) provides a cameo example of Ginette taking an opportunity to engage in some light relief with Helena by 'sending up' two male colleagues who had made silly mistakes. This kind of playful humour was something she also mentioned in the interview excerpt on humour within the team discussed in chapter 6 .

(10) Stupid eh?

Ginette yesterday + afternoon Christian and I were standing at the end by the elevator over there talking and David was coming round with the vacuum by the two kilo elevator + and just along the wall there on the ( ) there's a trail of powder just went right along + we were standing away talking and David had the hose and had that long thing connected t- hosing um vacuuming by the two $\mathrm{k} g$ elevator + and then he went over to clean that trail of powder + along side the wall + what he did h- he disconnected the hose off + off the end piece and then he walked over and he swept [voc] + the trail [laughs]:of powder up with that:

Helena how stupid

Ginette [laughs]: with that metal bit:

Helena yeah

Ginette when he finished that he connected the hose back on and then he vacuumed it up + the pile of powder that he'd swept up with just (the end) me and Christian were just cracking up laughing and (he turns to me) ( ) said + this is very [laughs]: embarrassing:

Helena [laughs]

Ginette I thought what a dick + you know all he had to do was go along with this thing and suck it all up

Helena and suck it up + it's actually easier $+(\quad)$ for that one $+\ldots \ldots$

Ginette dumb eh oh and it wa- I think it was yesterday or the day before + he had Sam up there there must have been a blockage in the hopper and Lesia and I were standing (and he) was banging away + I said to Lesia why the fuck is he banging the dust extraction pipe know that big thick pipe

Helena yeah

Ginette instead of banging the hopper// he was $=$ banging the pipe $/ /[$ laughs $] \backslash$

Helena /[laughs]\\/[laughs]\

Ginette [laughs]: and I said to him: what's the matter Sam [mimics Sam]: oh hopper's blocked powder's not coming through to the head: so why are you banging the dust extraction pipe [mimics Sam]: oh:

Helena [laughs] 
This is a good example of how Ginette would often enact a status-neutral 'team member' identity. The implicit invoking of gender stereotypes in this story (the domestically 'useless' male) also serves to position her as 'one of the girls' in a rare display of female solidarity. There are many other examples similar to this one in the data set, including several where the butt of the humour or gossip is someone outside the team, thus serving to reinforce the group boundary between the Power Rangers and other staff in the factory.

At the same time, participating in some no holds barred 'us versus them' gossip or humour also firmly positioned Ginette as 'one of us' rather than 'one of them'. This was significant given the amount of time Ginette spent away from her home base working with other factory staff, either on Power Rangers business, or on assignment to a range of special projects. In excerpt (11) Ginette was whingeing to Sue, another team member, that an administrative assistant (not a team member) had failed to do some laminating of training materials that she had requested.

(11) That dumb mole

Ginette $\quad$ that dumb mole that did my laminating ++ dumb bitch ++ Rylie + all the stuff I
want laminated she hasn't done it yet but she's done two copies of the ones down
there + I saw a whole heap and I thought oh yeah she's done it all and I've just been
through (it and) [high voice]: where the fuck's all that stuff: it was just double copies
of all the stuff that first stuff that I had got her to do + she did them again and didn't
do the stuff that I wanted ++ fuck man + said to Warren and Tim you can BLOODY
have her back [laughs]: I don't want her anymore: she's useless +
has Millie got any computer skills +
[drawls]: er: not really she's only basic like me + you can ask her but +
(she- ) bloody Rylie's useless \# she's got no computer skills + and Warren and Tim
Ginette $\quad$ were carrying on like she was an expert they just wanted to get rid of her
Sue
Ginette:

Excerpt (12) also shows collaborative power at work, but here it was enacted using a very different discursive strategy. This example illustrates how Ginette would sometimes code switch into Samoan ${ }^{5}$ to achieve or reinstate a collaborative footing, perhaps, as here, after earlier invoking her supervisory status as TCO, or simply as an expression of friendly solidarity, as we will see later in example (15).

Ginette's switch to Samoan (lines 8-11) helped to mitigate her potentially face threatening criticism of Lesia for not noticing the powder level was getting too low

\footnotetext{
${ }^{5}$ See Holmes and Stubbe 2004 for some further examples of how code switching is used in this workplace.
} 
(lines 1-4), while perhaps also softening her reminder to check the scoops (lines 910), by indexing it as an offer of assistance. This interpretation of how the switch into Samoan was functioning here is supported by Ginette's use of mate and that might be (the scoop) (line 9).

\section{(12) Not watching}

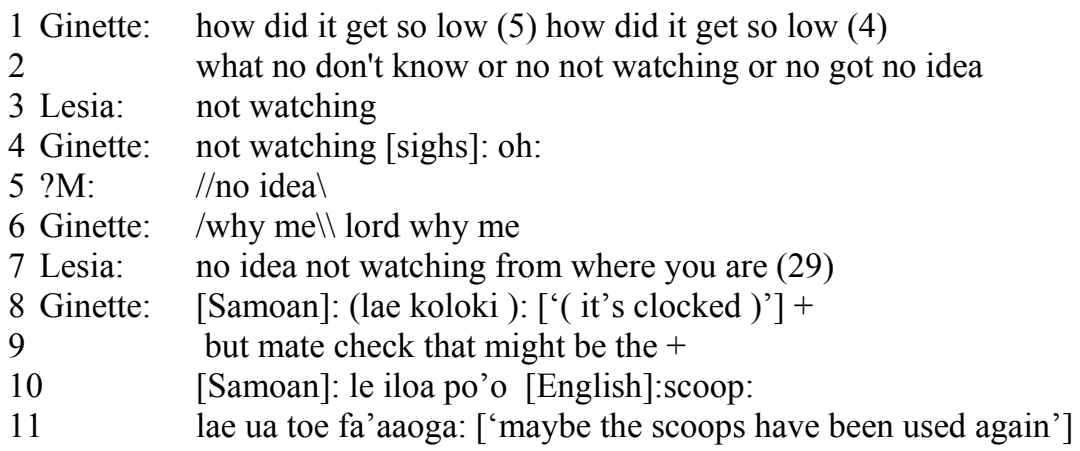

It is also interesting and significant that the earlier direct criticism and exasperated comment why me lord why me (line 6) were in English at a point in the exchange where Ginette was emphasising her status over Lesia; her choice of English can thus be seen as a distancing strategy. The later switch into Samoan may therefore also have functioned to reduce any distancing caused by the earlier interchange. At an instrumental level, the use of Samoan in this exchange functioned as a means of streamlining the solving of a practical problem on the packing line. Inevitably, however, use of a shared minority language in the predominantly English-speaking environment of the factory also functioned as a solidarity device, thus helping Ginette at the same time to position herself discreetly as 'one of the Samaons', and in this case to also mitigate a negatively affective speech act.

\section{The informal leader: 'first amongst equals'}

We have seen in a number of examples now how seamlessly Ginette shifted her footing, her interactional style, even the language she used, between an indexing of her formal role and her identity as an ordinary team member across different settings, or even within the same interactional sequence. In addition, Ginette also sometimes constructed a kind of middle position which allowed her to subtly exploit and reinforce aspects of both these facets of her identity. In such cases she still seemed to be enacting her leadership, but she did so in a more informal style which drew on her personal influence, and on the consensual types of power (empowerment and collaboration) to position herself as 'first amongst equals'. One example of this was 
the way she regularly deferred to and supported other team members with delegated responsibilities in briefing sessions and on the factory floor. Another was her encouragement of mutual engagement and a positive team spirit, in particular through the use of humour. This included using the team newsletter to recount funny stories or start up a running joke (such as the one about weight watching we have seen traces of in earlier examples), setting the team 'brainteaser' puzzles to solve in the course of a day or shift, or instigating a practical joke of some kind.

Although also offering another opportunity for her to relax and have fun with her coworkers, she reported that her involvement in these latter types of activities were consciously designed with the specific purpose of building motivation and camaraderie within the team, and generally relieving the monotony of the daily work. As such, it fell within the remit of her role as TCO. Therefore, as well as involving elements of collaborative power, her facilitation of these kinds of social interaction could also be interpreted as the enacting of reward power, in the sense that she was 'giving permission' for social talk and activities to carry on in work time. (This was always on the clear understanding that the work came first, as stated in John's interview in chapter 6.) One classic case occurred on April Fools' Day when she tricked several team members into ringing the local zoo to ask for "Mr Lion", much to the mirth of their colleagues.

Sometimes, in addition to licensing off-task talk, more tangible 'rewards' were also offered as a way of building team cohesion, as in the next two brief examples. In excerpt (13), Ginette was joking to some of the men in the manufacturers' control room about the reactions of some of the packers when she handed out Easter eggs.

(13) Easter eggs

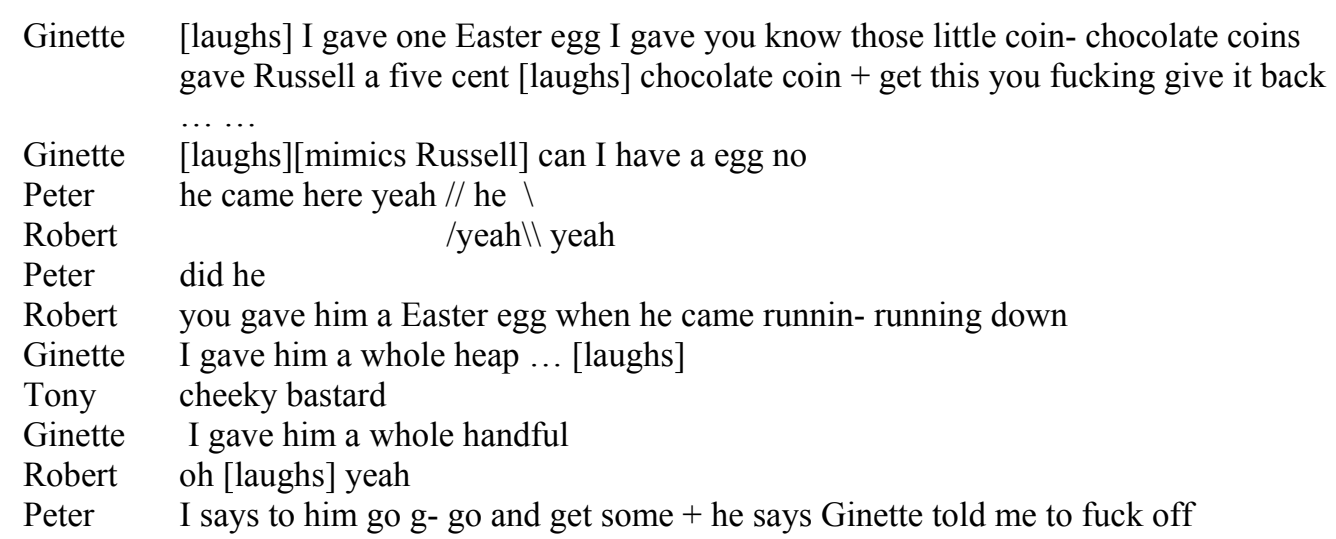


This was clearly off-topic talk, initiated by Ginette, and it continued for some time. The Easter break was approaching and there was a bit of downtime in the production cycle so social talk was tolerated and even encouraged. In excerpt (14) Ginette appears to be giving her standard kind of briefing, with a string of injunctions delivered straight-faced to the team. However, on closer listening, it becomes apparent that she was offering them each a semi-official 'memento' of the last ever cases of a discontinued product line. The instructions were genuine, but because this was not strictly a work activity as such, Ginette's style of delivery indexes this in another example of hybrid identity work.

\section{(14) Mementos}

Ginette the very last twenty five cases that you take off that line I want them put aside
the very last twenty five cases put them on a pallet
get them stretch wrapped
theyre going to be a memento for everybody
so make sure you er remember that ...
so just remember the last the very last twenty five cases put them on a pallet
get them stretch wrapped
put them aside for er [name] ...
send them through with no glue

\section{Power and solidarity: exploiting multiple meanings}

The final example ${ }^{6}$ provides a cameo view of the way Ginette skilfully enacts both power and solidarity in a longer interactional sequence as she juggles her dual workplace 'hats' as manager and member of this close-knit, multicultural factory team, while at the same time constructing her personal identity as a New Zealand Samoan woman. Here she was doing her 'rounds', and had stopped to talk to Lesia, a leading hand and one of the workers on the packing line. Lesia kept on working throughout the interaction, moving back and forth between a stack of empty boxes and the bench where he was packing.

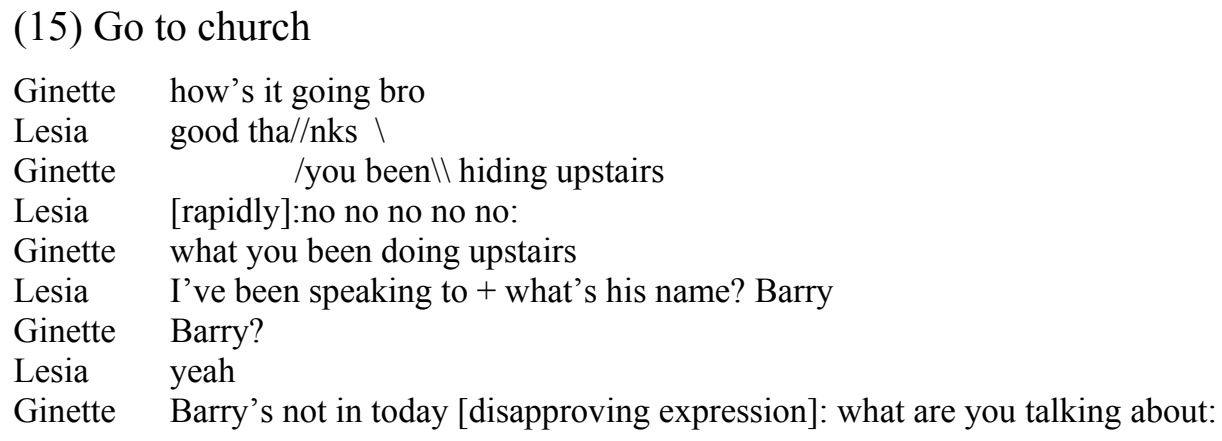

\footnotetext{
${ }^{6}$ This interaction was captured on video as part of the development of the video resource mentioned earlier (Stubbe and Brown 2002).
} 


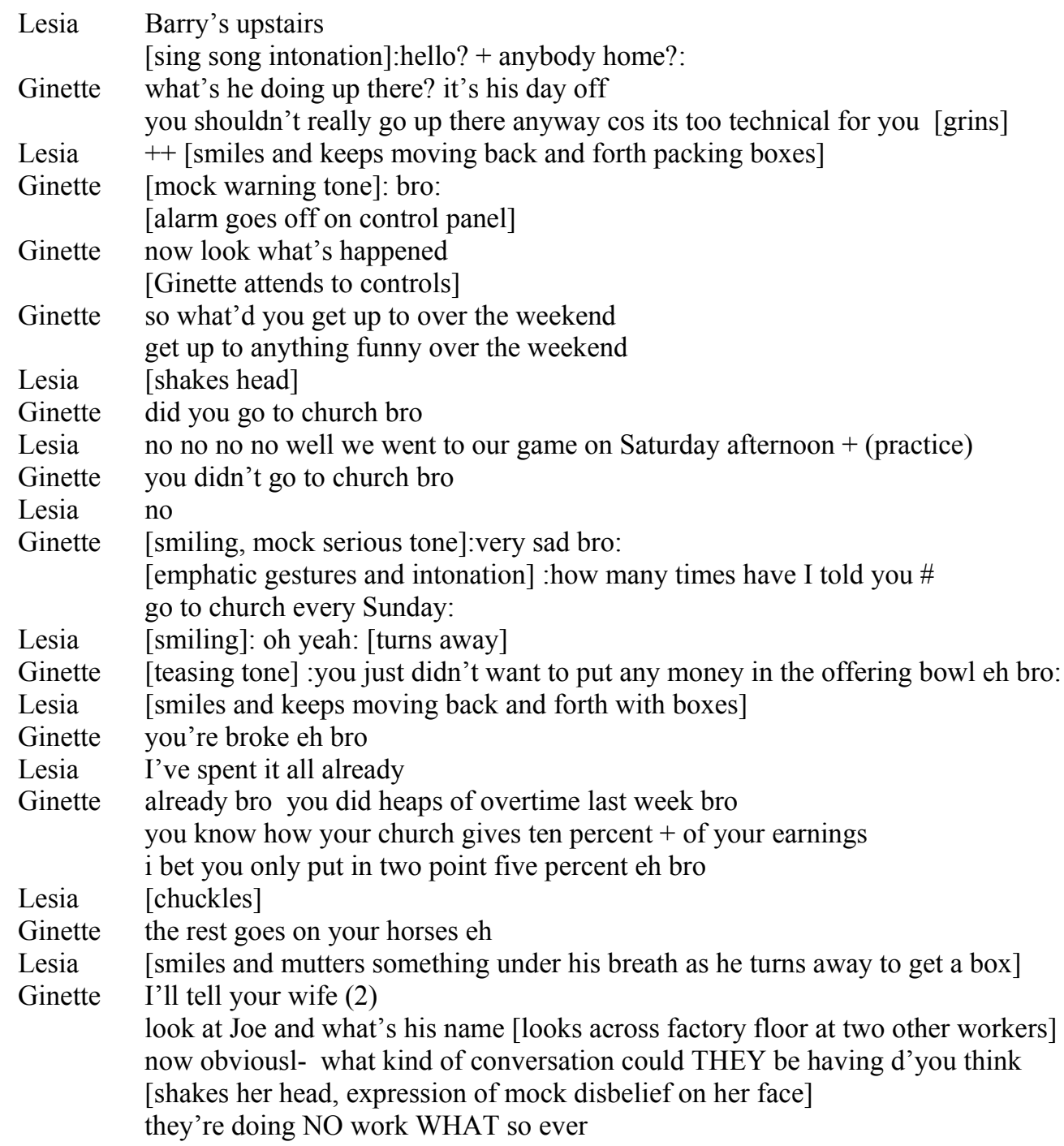

While Ginette often adopted a very direct, almost authoritarian style, humorously alluded to here with her mock serious berating of Lesia (how many times have I told you \# go to church every Sunday), we see here how this was tempered and blended with a range of both collaborative and contestive discourse strategies as she teased Lesia about the reasons for his supposed lack of generosity. Here she also indexed their shared Samoan ethnicity and cultural identity, which is traditionally closely bound with regular churchgoing and matriarchal influence over the extended family's behaviour and moral code. In these ways, as we have seen her doing in other examples, Ginette worked to build solidarity and to minimise the difference in status between her and Lesia at one level, while at the same time subtly indexing her authority via the identity position of 'the matriarch' or 'Camp Mother'. She then neatly switched the conversation back to a business footing with her comment about the two workers who, unlike Lesia, were off-task while chatting (what kind of conversation ...WHAT so ever). This comment both underlined her status as team 
manager and indirectly reinforced her expectations of how team members should conduct themselves.

This excerpt nicely encapsulates many of the ways in which we have seen Ginette talking to other members of her team in the course of this chapter - by turns bossy, giving direct instructions, joking and being supportive and nurturing - as well as the way she very consciously looks after their practical and emotional needs. The discourse strategies she uses are all mutually reinforcing, but they can often be interpreted in a number of different ways in terms of the roles, identities and types of power associated with them. Instead of being problematic, these inherent ambiguities provide Ginette with a rich resource to help her manage the contradictions between different aspects of her identity as TCO of the Power Rangers team.

\subsection{Conclusion}

Effective communication and positive relationships are widely acknowledged to be the foundation for good teamwork and sound leadership, a truism that this case study has so far reaffirmed. As we have seen, in a high-stakes challenging production line environment such as the one the Power Rangers team worked in, miscommunication and conflict were key risks. Consequently, managing miscommunication and other kinds of problematic discourse on a day to day basis, as well as optimising team relationships and interactions, was of crucial importance to the TCO in her role of ensuring the smooth operation of the team's core business.

The particular ways in which Ginette achieved this outcome would not generally be considered to be a 'textbook model' of effective workplace communication. Nevertheless, as the various analyses above have illustrated, Ginette's skilful management of a complex discursive repertoire allowed her to assert control where required, while also paying explicit attention to the face needs of her interlocutors and the social environment of the team as a whole. Through the skilful use and interplay of a range of authoritative, contestive and collaborative strategies, she simultaneously aligned with and further reinforced the high solidarity culture characteristic of this particular community of practice. At the same time, the different identity positions she adopted provided her with a further resource to mitigate overt displays of power where these were called for, and reinforce values of team cohesion 
and social connection. She played down her individual status as team leader, choosing instead to influence and carry the team along with her in other more inclusive ways, something which is a typical feature of Polynesian culture as well as a commonly cited aspect of the feminine end of the communicative style continuum. The multiple meanings inherent in this team leader's discourse thus provided her with a powerful strategic resource in trying to balance the often contradictory demands of her particular workplace situation. 


\title{
8 Problem or paradox? The elephant in the room
}

\begin{abstract}
A senior manager explains her criteria for selecting job applicants in her section
... we've chosen people who are really good open confident communicators at a business level and at an interpersonal level ... so hopefully we've moved the culture ahead a step further by the selection of those four people ... obviously we met all the technical competencies we needed but largely we made the choice amongst a HUGE pool of really good applicants on interpersonal skills ... and communication being a big part of that - their way of working.
\end{abstract}

\subsection{Introduction}

Managing miscommunication and problematic talk at work (or in any other social context for that matter), means coming to grips with a fundamental paradox: namely, rather than being somehow the opposite of good or effective communication, miscommunication is in fact an inevitable consequence and, arguably, an essential component of the richness and complexity of human interaction. There are many fascinating theoretical and philosophical issues that arise out of this observation, and the review of the literature and analysis of workplace data in this thesis has shown clearly that there is a great deal of scope yet for these kinds of explorations to continue well into the future. There can be no doubt, then, that miscommunication and problematic talk are very complex and multi-faceted phenomena indeed, no less so than the overall system of communication of which they are an integral part.

However, it is also true that these problematic aspects of human communication represent a significant occupational hazard of working life in the here and now, with the potential to lead to many different kinds of negative outcomes ranging from trivial and insignificant irritations to more serious interpersonal, economic or even life-threatening consequences. Moreover, as illustrated in the quotation that opens this chapter, there is an increasing demand by employers that workers at all levels and in all occupational groups should have well-developed communication and 
teamwork skills which are seen as providing an essential foundation for both corporate productivity and individual success, and are often considered to be equal in importance to technical or professional competencies.

As a researcher who chooses to bridge the divide between theory and practice, the central question for me when I first conceived of this project was how we might theorise miscommunication and problematic talk in a way that can be translated usefully (and more immediately) into a set of practical tools. In other words, was it possible to construct a theoretically robust 'working model' that people in workplaces, and the researchers or advisors working alongside them, could learn to use as a framework for analysing and hence better 'managing' real world cases of problematic discourse?

To meet this ultimate objective, it was necessary first to gain an understanding of what models and tools were already available across a range of disciplines, theories and methodologies, and to determine whether it would be possible or useful to try to construct some kind of integrated theoretical framework. It seemed sensible to articulate this theoretical exploration with some descriptive fieldwork and analysis to start building up a picture of the range of communication problems that typically occurred (or were perceived to occur) in the New Zealand workplaces that were already participating in a wider study of workplace language, and to look more particularly at the strategies the people in those workplaces used to prevent or repair miscommunication and problematic talk.

As explained in chapter 1, it quickly became clear that taking a narrow approach to defining or analysing instances of miscommunication would not be particularly helpful either for the purposes of a theoretical descriptive analysis or to resolve the kinds of practical issues our research partners were interested in finding solutions for. As discussed in chapters 4 and 5, a more inductive and context-sensitive approach was therefore adopted to meet their needs for practical applications of the research process as well as to meet my own research objectives. As also discussed in the analysis of exemplar cases in chapter 5, another unexpected finding was that just as many useful and interesting things could be found out about miscommunication and problematic talk by looking at instances of routine or clearly effective interaction, as by trying to purposively identify and capture problematic instances. Although my process was taking place at a different level of detail, this finding interestingly 
reflects and affirms the value of the ethnomethodological/conversation analysis practices of 'unmotivated looking' and analysing 'mundane' events.

These interests crystallised into the three overall research objectives for this study, as discussed in the introductory chapter, and into the claim that has been addressed in this thesis. In short, I have proposed that in order to comprehensively address 'real world' cases of workplace miscommunication and problematic talk in all their complexity, it is necessary to take a pluralistic multi-layered intertextual approach which incorporates multiple viewpoints, analytic methods and data sources. I have explored this proposition by means of an inductive analysis of a large corpus of workplace interaction data, represented here by the development of a working analytic framework as explicated in chapter 5, and by the in depth analysis of three exemplar cases drawn from that baseline data set using that framework.

This was followed by in an intensive longitudinal case study of the strategies used to manage communication, miscommunication and problematic talk in a factory production team as reported in chapters 6 and 7 . This added three further elements to the analytic model, made possible by the intensive case study research design. The first of these added elements was to analyse miscommunication and problematic talk in the context of a discursive community of practice framework in order to strengthen the sensitivity of the analysis to contextual and situational factors. The second was to introduce a multiplex longitudinal tracking design which greatly enriched the interpretation of the data according to the different dimensions and levels of analysis identified in the analytic framework. The final added element was made possible by the first two, which was to undertake a comprehensive analysis from a critical perspective of the use of identity resources in the negotiation of problematic talk. 


\subsection{Summary of key findings}

As reported at the end of chapter 3, the analysis of these data sets was guided by the following specific research questions (reproduced here for convenience):

1. What kinds of workplace communication problems can be observed to occur in the New Zealand case study data? And what discursive strategies do these workplace participants use to prevent, manage and repair miscommunication and problematic talk in their everyday interactions?

2. What issues arise when we attempt to identify, classify and analyse examples of miscommunication and problematic talk in a corpus of naturalistic workplace talk?

3. Does a pluralistic multi-layered case study approach 'add value' to the analysis?

Firstly, both the international literature and the New Zealand data analysed for this study revealed a very wide range of communicative actions or features that can qualify as problematic in one way or another. This can include relatively straightforward examples where it appears that the content or message has not been accurately conveyed, although such explicit examples were in fact very rare in the corpus of workplace talk studied here. (As discussed previously, this may reflect a capture problem as well as, or instead of, indicating the actual frequency of such types of miscommunication.) Cases of problematic talk involving relational and power issues were also common in the data, as reflected in a number of the examples analysed in chapters 5 to 7 in this thesis. The analytic framework presented in chapter 5 provides a comprehensive summary of the different types of miscommunication and problematic talk and related factors and dimensions that $\mathrm{I}$ identified from my review of the research literature and cross-sectional inductive analysis of the baseline data set.

Secondly, as already discussed above, another clear conclusion derives from the fact that miscommunication and problematic talk can be demonstrated to be ubiquitous aspects of the communication process. Because of the complexity and 'fuzziness' of language and interaction, the potential for problems to occur is always there, and people are therefore always 'managing' this risk at one level or another. As we saw in chapter 3 and again in chapters 5, 6 and 7, it then often becomes a matter of where one draws the line definitionally as to whether a particular (possibly successful) instance of an interactional strategy being used should 'count' as an example of 
either 'problematic' or 'effective' talk. In some cases this is quite straightforward to decide, but in practice, this kind of categorisation is often unhelpful. It makes more sense from the perspective of the end-user to see different kinds of phenomena on a continuum of more or less effective communication (and how one defines 'effective' of course also very much depends on the context and the perspective of the participants).

Conversely, the literature review and data analyses have also demonstrated that the kinds of strategies adopted to manage different kinds of problematic talk can vary a great deal and can take place at any of a number of linguistic or discursive levels. As demonstrated in a number of the examples analysed here, at the level of the single interaction these might, for example, include pragmatic devices such as hedges, attenuators, boosters and intensifiers of various kinds which along with other discourse strategies such as avoidance or supportive feedback or humour can serve to mitigate potential threats to face. Speakers also make use of more macro-level discursive resources such as avoidance of face-threatening acts, different kinds of turn or sequence design, appeals to institutional procedures and processes and strategic management of different roles and identities. And as we saw in chapter 5, even the possible existence of miscommunication can be used as a strategic resource in the management of problematic talk.

Another important point arising from the analysis of the data is that the strategies used to avoid conflict and maintain good workplace relations are just as evident in individuals' management of many other kinds of problematic talk. It is therefore seldom a simple matter to locate the precise point at which miscommunication or problematic talk can be said to have occurred. The analysis, whether from the perspective of participant or analyst, always relies heavily on contextual information and ethnographic knowledge at a number of different levels, and must always be seen in the light of wider societal discourses. Miscommunication and other forms of problematic discourse thus have to be seen and analysed in the wider context of the 'discursive fabric' in which they are enmeshed - even the way quite localised misunderstandings play out is influenced by this wider context, which includes the shared practices and history of the individuals involved.

The most effective workplace communicators seem to be those like Ginette, the team coordinator at the soap factory, or Jan the policy manager, who understand the 
complex balancing acts that are involved at some level in every communicative sequence and context, who are constantly alert to the potential for things to go wrong, and who are therefore proactive in monitoring and managing the possible risks. These effective communicators seem to operate according to an intuitive understanding that workplace communication operates within a complex network of interrelated dynamic systems and practices, which are mutually influencing and interdependent. Effective 'managers' of miscommunication and problematic talk therefore also tend to be highly effective communicators more generally, and effective in their interpersonal dealings as well.

As we have seen, there is much to learn by systematically observing how key players like these optimise the communicative environments and processes in which instances of problematic talk are embedded. We also need to understand what is 'functional' about apparently dysfunctional communication practices and processes or patterns of interaction (or sometimes vice versa), and what the costs or benefits might be of 'fixing' these without regard to what motivates them, or to the structural and systemic influences and constraints of a particular context (cf. Silverman 2001).

Paradoxically once again though, it is often easiest to identify effective communication strategies at times when something problematic is going on, so close analysis of clear cases is also important. However, as has also been seen repeatedly throughout this thesis, it is not always straightforward to identify whether a given incident has problematic elements or not, and any analysis is hugely complicated by the fact that there are many overlapping, sometimes cross-cutting, dimensions and factors to consider in any given case. If we stopped to think about it, rather than being surprised at how often something goes wrong with a communication process, we should be surprised at how often they go right, or potential problems are retrieved, repaired or deflected.

Given the degree of complexity involved it seems fair to conclude that not only does a pluralistic multi-layered case study approach 'add value' to the analysis in terms of theoretical/ methodological rigour and practical applicability, but such an approach is in fact absolutely essential for analysing and dealing effectively with the multidimensional reality of 'whole cases' of miscommunication and problematic talk at work. Some of the theoretical and practical implications of these findings are discussed next. 


\subsection{Implications and applications}

As already alluded to, the main theoretical implication of this study is that the "holy grail' of a single integrated conceptual model of miscommunication and problematic talk is neither achievable, nor likely to be especially useful; given the sheer complexity of these phenomena, any such attempt is likely to result in a reductionist model which would not meet the practical objectives of workplace practitioners nearly as well as a more pluralistic model. As discussed in chapters 2 and 3, the modelling of language and communication as a type of complex adaptive system is just beginning to gain some currency. Its applicability to the study of miscommunication and problematic talk in workplaces is an avenue that remains to be explored in greater depth, but even so, it is clear that certain concepts from complexity theory do seem applicable as a way of framing the multi-dimensional concepts we have been grappling with here.

In particular the notion of incompressibility seems to have some bearing in this case. As Cilliers defines it: “(w)e have seen that there is no accurate (or rather perfect) representation of the system which is simpler than the system itself" (2005:13). Essentially, this suggests that "the best representation of a complex system is the system itself, and any representation of the system will be incomplete and, therefore, can lead to incomplete understanding" (Richardson 2008:16). Richardson goes on to suggest that although a degree of fragmentation is inevitable and can be useful, a pluralist approach in which "many theories coexist each with their own strengths and weaknesses" is preferable to insisting on a unifying framework which simply papers over the cracks (Richardson 2008:16).

As we saw in the conclusion to chapter 3, this view is not limited to complexity theorists, with a number of applied linguists in particular taking the stance that no single approach on its own provides a fully adequate account of various communicative phenomena. Some management theorists such as Donald Schon also argue that professional situations are unique and often complex, and that standardised applications of generalised theories are therefore not effective. Multiparadigmatic models based on reflection-in-action such as the communication evaluation and development (CED) model discussed in chapters 4 and 5, provide an alternative by allowing a contextual reading of a problem which puts the experiences and tacit knowledge of the practitioner at the centre (Jones and Stubbe 2004). The 
pluralist approach as adopted in this thesis has certainly provided the kind of flexible and expansive explanatory framework for analysing actual workplace interactions that has been called for by these researchers. In fact, a multi-paradigm approach was not only helpful, but almost impossible to avoid in trying to account for the diversity found in the data. I would therefore reiterate that although this has inevitably increased the complexity of the analysis, comparing and combining different approaches in this manner has yielded a richer and more comprehensive analysis in the case of the present study.

In terms of methodology, there is no doubt that for the purposes of providing an intertextual, situated thick description, a comprehensive longitudinal case study design is the gold standard, especially in workplace settings. The workplace has particular features which may add further layers of complexity, in particular localised sets of practices, activity types and institutionally ordered relationships which may be both interactional and 'practical' (with the latter to a very large extent instantiated through the medium of talk). This is not to say that useful insights cannot be gained from more randomly configured data sets; clearly, the baseline data analysis which relied on far more partial data nevertheless provided a great deal of grist to the analytic mill. But as we saw with each of the exemplar cases, the fragmented nature of the data did impose limitations on what could be analysed and to what level of detail. The lack of data triangulation also made it more difficult to be confident of which of a number of possible interpretations to favour on a number of occasions.

The extended case study of the Power Rangers team reported in chapters 6 and 7 will repay further discussion at this point to tease out some of the practical implications of the research reported in this thesis. This case study was motivated by a 'real world' problem or question posed by the factory management - 'How does this team's communication style relate to their success, and what can we learn in order to help other less functional and productive teams improve their performance?' This question had an added degree of piquancy because, as we saw, the communicative and social culture of this team was contrary in some respects to 'received wisdom' and accepted norms of behaviour. The analysis demonstrated that members of the Power Rangers team faced many interrelated communicative challenges and demands, but at the same time had a number of effective communicative processes 
and strategies in place which contributed in no small measure to their performance as a well-motivated and high-performing production team.

A second objective of the original action research was to try and tease out which of the communication features and strategies observed were unique to this particular team, and therefore not readily transferable to other groups, and which ones could possibly be identified and emulated successfully by other teams in similar contexts. This is a rather more complex issue, one that applies equally to the features and strategies relating to miscommunication, and requires more detailed analysis than is possible here. However, it is possible to draw some initial conclusions based on the analyses presented in chapters 6 and 7 .

It is obvious from the case study that the 'Power Rangers' had developed a particular style of communication that suited the individuals in the team and which had evolved out of a unique interaction of personal and group characteristics. For instance, the TCO played a crucial role in uniting the team around a common purpose, as well as fostering and modelling the use of a wide range of communication strategies, including the use of humour. It is also likely that the cultural composition of the team, with its high proportion of Polynesian workers, was a major influence on the development of the team's 'high solidarity' style of interaction, and the sense of collective responsibility that was evident. For these reasons, amongst others, it is unlikely that another team could simply have copied the Power Rangers' way of doing things, even if this were seen as desirable.

As we saw in chapters 6 and 7, a more useful way of looking at these issues is to see the team as a discursive community of practice, with its own unique 'culture' or ethos and a shared repertoire of communicative strategies. This framework implies that there is no one 'right' way to do things, and that taking a prescriptive, skillsbased approach will not get us very far. Rather, each team will need to develop its own way of communicating and managing communication problems and challenges, and its own set of shared practices which reflect the needs, skills and personal characteristics of its members. 


\subsection{Concluding remarks}

This study represents a novel approach to workplace communication research both in terms of the methodology used, and in the way in which the multi-dimensional analytic framework was developed in partnership with workplace participants who were engaged in a communication evaluation and development programme. This programme was based on the principles of participatory action research and reflective practice which also inspired and partly dictated the inductive iterative analytic process that was followed. The data collection and analytic methodologies were originally devised as part of the wider Language in the Workplace Project which recorded a limited number of 'one-off' interactions from workers in a range of professional workplaces. Although this earlier research was not designed for the purposive collection of related interactions, the few that were sometimes collected by chance, and the action research process itself, showed the potential value of examining intertextual links and taking an intensive longitudinal case study approach to the proposed study of miscommunication and problematic talk.

The intensive and holistic approach to data collection and analysis developed as part of this thesis project has made it possible to develop a 'thick description' based on interactional sociolinguistic principles, and thus to relate what was happening during particular workplace interactions to wider issues such as team or organisational culture, group membership and the wider socio-cultural context.

The deliberate comparison of insights from micro- and macro-level analyses focusing on different aspects of the same data provided an opportunity to identify generalisable and more widely applicable themes and patterns within a community of practice framework, as well as offering an opportunity for rich and multiplex analysis of individual interactions or sets of interactions. This allowed a more nuanced analysis than would be possible by using a single approach or data type. It also made possible an integrated grounded approach to the analysis of individual cases and a systematic description of team-wide practices, which made it much easier to identify and reliably interpret the cases of miscommunication and problematic talk which emerged from the data. The communication evaluation and development model which developed progressively out of and alongside this analytic framework has also proved to be a useful and practical means of helping workplace participants develop a greater awareness of the range of discourse strategies and processes they might use 
to assist them in selecting appropriately from the linguistic and other resources they have available to them.

This brings us back to the proposition put forward in the introduction to this thesis; namely, that in order to take adequate account of the dynamic multi-faceted nature of miscommunication and problematic talk, and of the many levels and contexts in which these can occur, the most constructive approach is to adopt the kind of analytic framework and methodology proposed here, which draws on the insights and approaches of a number of different theoretical perspectives in an explicitly multiparadigm 'toolkit' approach. The overall conclusion is that given such a diversity of perspectives and tricky analytic issues to grapple with, it is neither especially helpful nor realistic to attempt to articulate the many possible theoretical conceptualisations of miscommunication and problematic talk into a single and coherent 'integrated model' - at this stage at least, 'the elephant in the room' may simply be too big to fit into a single frame of this kind. 


\section{Appendix}

\section{Transcription conventions}

\begin{tabular}{|c|c|}
\hline YES & Capitals indicate emphatic stress \\
\hline [laughs]: : & Paralinguistic features (colons indicate start/finish) \\
\hline+ & Pause of up to one second \\
\hline (3) & Pause of specified number of seconds \\
\hline$\ldots / \ldots \ldots 1 \ldots$ & Simultaneous speech \\
\hline$\ldots / \ldots \ldots . . \ldots$ & \\
\hline (hello) & Transcriber's best guess at an unclear utterance \\
\hline$:::$ & Lengthened sound (number of colons indicates extent) \\
\hline$?$ & Rising or question intonation \\
\hline- & Incomplete or cut-off utterance \\
\hline , & continuing intonation \\
\hline$\#$ & Emphatic sentence-final intonation \\
\hline$\ldots$ & Section of transcript omitted \\
\hline
\end{tabular}

All names used in examples are pseudonyms. 


\title{
2 Research documentation
}

\author{
(a) Information sheet and consent form
}

\section{Language in the Workplace Project \\ Information for participants}

\section{About the project}

Researchers from the Language in the Workplace Project have been studying workplace communication since 1996. So far, we have collected hundreds of recordings from office workers in government departments and companies in Wellington like [name] and [name], from factory workers in Auckland, and from various small businesses in Hawkes Bay. Some aspects of workplace talk we have looked at include how people use talk to get things done at work, how people prevent or fix up misunderstandings, and how they use humour and small talk to get on better with their workmates.

\section{What will the researchers do?}

At [name of organisation], we want to find out what strategies are used by groups of workers that communicate well, and then use this information to help teams find ways to improve the way they work together. To do this we need to find out how people actually talk to each other as they go about their work. One of our researchers will observe and talk to the members of your team and make notes, and some of your colleagues will record some of their day-to-day interactions onto (audio/video tape/disc). We will then take these recordings away, transcribe them, and analyse the communication patterns. (We will replace real names with pseudonyms to protect your identity). When we have finished, we will give you a summary of the results, and check whether you would like any other sort of feedback, such as a workshop.

What will the data be used for?

The recordings and other information we collect from you will be used only for these purposes:

(i) linguistic research, including doctoral research; (ii) publications, presentations and resource materials based on this research; and (iii) evaluation and development of team communication at [organisation].

\section{Who will have access to the data?}

All tapes and other information collected as part of this project will be stored securely at Victoria University. Only authorised members of the research team will have access to this material. From time to time, we may play short excerpts from the tapes in professional contexts such as seminars, but only if we are sure that no one will recognise you. We will not play any recordings to other staff or managers at [organisation], or put them to any commercial use without your express permission.

I give permission for audio/video recordings of my talk at work and other related information collected by the Language in the Workplace Project at [organisation] from [dates] to be used for the research purposes described above. I understand that only authorised members of the research team will have access to the tapes and any personal information collected as part of this project, and that my identity will not be disclosed without my permission in any circumstances.

Signed: Full Name: Date: 


\section{Language in the Workplace Project \\ Background Information}

1. What is your current job?

2. How long have you been with [organisation]?

3. Gender:

$\square$ Female $\square$ Male

4. Circle your age group:

$$
\begin{array}{llll}
16-19 & 20-24 & 25-29 & 30-34 \\
35-39 & 40-44 & 45-49 & 50-54 \\
55-59 & 60-64 & 65-69 &
\end{array}
$$

5. How old were you when you left school?

6. What is your highest educational qualification

7. Which ethnic group do you identify with?

8. Were you born in New Zealand?

$\square$ Yes $\square$ No

If yes, please specify town or region

If $\underline{\text { no}}$,(a) where were you born?

(b) at what age did you come to New Zealand?

9. Have you been overseas in the last 3 years?

$\square$ Yes $\square$ No

If yes, for how long were you overseas in the last 3 years?

years months weeks

How long in total have you spent overseas during your life? years months weeks

Provide approximate dates.

10. Do you speak any languages other than English?

$\square$ Yes $\square$ No If yes,

(a) Which language did you speak first in your home as a child?

(b) At what age did you first learn English?

(c) Which language(s) do you speak at home now?

(d) Which language(s) do you speak at work? 


\section{(c) Ethical Approval}

Ethical approval for the original Language in the Workplace Project and for the subsequent projects carried out under its umbrella, including the factory study reported on here, was granted by the Victoria University Human Ethics Committee prior to the commencement of each project or sub-project. The research agreements with participant groups at each workplace were based on the common information sheet and consent form in (a) and (b) above. Additional undertakings were made separately at each workplace as applicable in relation to specific caveats on the use of data, the agreed nature and timing of feedback on research results and participation in the piloting of communication evaluation and development materials. 


\section{References}

Adler, R., Rosenfeld, L. B., and Towne, N. (1996). Understanding Human Communication. Fort Worth: Harcourt Brace College Publishers.

Alvesson, M., and Karreman, D. (2000). Taking the linguistic turn in organizational research: Challenges, responses, consequences. Journal of Applied Behavioural Science, 36, 136-158.

Angouri, J., and Harwood, N. (2008). This is too formal for us. A case study of variation in the written products of a multinational consortium. Journal of Business and Technical Communication, 22(1), 38-64.

Anolli, L., Ciceri, R., and Riva, G. (Eds.). (2002). Say Not to Say. New Perspectives on Miscommunication. Amsterdam: Ios Press.

Antonacopoulou, E., and FitzGerald, L. (1996). Reframing competency in management development. Human Resource Management Journal, 6(1), 27-50.

Arford, P. H. (2005). Nurse-physician communication: an organizational accountability. Nursing Economics, 23(2), 72-77.

Argyris, C., and Schon, D. A. (1995). Organizational Learning II: Theory, Method and Practice: Prentice Hall.

Aronsson, K. (1991). Social interaction and the recycling of legal evidence. In N. Coupland, H. Giles and J. M. Wiemann (Eds.), "Miscommunication" and Problematic Talk (pp. 215-243). London: Sage Publications.

Auburn, T. (2005). Narrative reflexivity as a repair device for discounting "cognitive distortions" in sex offender treatment. Discourse and Society, 16(5), 697-718.

Bakhtin, M. (1981). The Dialogic Imagination: Four Essays. (Translated by C. Emerson and M. Holquist). In M. Holquist (Ed.). Austin: University of Texas Press.

Bakhtin, M. (1986). Speech Genres and Other Late Essays. (Translated by V. McGee). In C. Emerson and M. Holquist (Eds.). Austin: University of Texas Press.

Bargiela-Chiappini, F., and Harris, S. J. (Eds.). (1997a). Managing Language: The Discourse of Corporate Meetings. Amsterdam: John Benjamins.

Bargiela-Chiappini, F., and Harris, S. J. (1997b). Meetings as genre. In F. Bargiela-Chiappini and S. J. Harris (Eds.), Managing Language: The Discourse of Corporate Meetings (pp. 205-225). Amsterdam/Philadelphia: John Benjamins. 
Bargiela-Chiappini, F., and Harris, S. J. (1998). The Languages of Business: An International Perspective. Edinburgh: Edinburgh University Press.

Bateson, G. (1972). Steps to an Ecology of Mind. New York: Ballentine.

Bavelas, J. B. (1985). A situational theory of disqualification: Using language to "leave the field". In J. Forgas (Ed.), Language and Social Situations (pp. 189211). New York: Springer.

Baxter, J. (2003). Positioning Gender in Discourse: A Feminist Methodology. Basingstoke: Palgrave Macmillan.

Baxter, J. (2008). Is it all tough talking at the top? A feminist post-structuralist analysis of the construction of gendered speaking identities of British business leaders within interview narratives. Gender and Language, 2(2), 193-218.

Bazerman, C. (1994). Systems of genres and the enactment of social intentions. In A. Freedman and P. Medway (Eds.), Genre and the New Rhetoric (pp. 79-101). London: Taylor and Francis.

Bazerman, C., and Russell, D. R. (Eds.). (2003). Writing Selves/Writing Societies: Research from Activity Perspectives. Colorado State University, Fort Collins, Colorado: The WAC Clearinghouse, http://wac.colostate.edu.

Bazzanella, C., and Damiano, R. (1999). The interactional handling of misunderstanding in everyday conversations. Journal of Pragmatics, 31(6), 817836.

Belfiore, M. E., Defoe, T. A., Folinsbee, S., Hunter, J., Jackson, N. S., and Hunter, J. M. (Eds.). (2004). Reading Work: Literacies in the New Workplace. Mahwah, NJ: Lawrence Erlbaum.

Bell, A. (1991). Hot air: media, miscommunication and the climate change issue. In N. Coupland, H. Giles and J. M. Wiemann (Eds.), Miscommunication and Problematic Talk (pp. 259-282). London: Sage.

Berger, P. L., and Luckman, T. (1966). The Social Construction of Reality: A Treatise in the Sociology of Knowledge. New York: Doubleday.

Bergvall, V. L., Bing, J. M., and Freed, A. F. (1996). Rethinking Language and Gender Research: Theory And Practice. London and New York: Longman.

Bhatia, V. K. (2002). Applied genre analysis: a multi-perspective model. IBÉRICA, 4, 3-19.

Bilmes, J. (1992). Mishearings. In G. Watson and R. M. Seiler (Eds.), Text in Context: Contributions to Ethnomethodology (pp. 79-98). London: Sage.

Bing, J. M., and Lombardo, L. X. (1997). Talking past each other about sexual harassment: an exploration of frames for understanding. Discourse and Society, $8(3), 293-311$.

Bleakley, A. (2006). A common body of care: The ethics and politics of teamwork in the operating theater are inseparable. Journal of Medical Philosophy, 31(3), 305-322.

Boden, D. (1994). The Business of Talk: Organizations in Action. Cambridge: Polity Press. 
Bou-Franch, P. (2002). Misunderstandings and unofficial knowledge in institutional discourse. In D. Walton and D. Scheu (Eds.), Culture and Power (pp. 323-341). Bern: Peter Lang.

Britten, N. (2004). Patients' expectations of consultations. British Medical Journal, 328(7437), 416-417.

Britten, N., Stevenson, F., Barry, C., Barber, N., and Bradley, C. (2000). Misunderstandings in prescribing decisions in general practice: a qualitative study. British Medical Journal, 320(7233), 484-488.

Brown, P. (1998). Directives in an Auckland factory. Te Reo: Special Issue Proceedings of the Sixth Language and Society Conference June 1998, 41, 199202.

Brown, P. (2000). "Might be worth getting it done then": Directives in a New Zealand Factory. Unpublished MA (Applied) Thesis, Victoria University of Wellington, Wellington.

Brown, P., and Levinson, S. (1978). Universals in language usage: politeness phenomena. In E. N. Goody (Ed.), Questions and Politeness. Cambridge: Cambridge University Press.

Brown, P., and Levinson, S. (1987). Politeness: Some Universals in Language Usage. Cambridge: Cambridge University Press.

Brown, T. P., and Lewis, M. (2003). Workplace language: Talking with the pay clerk. English for Specific Purposes 22(1), 93-98.

Butler, J. (1990). Gender Trouble: Feminism and the Subversion of Identity. New York: Routledge.

Cameron, D. (1995). Rethinking language and gender studies: some issues for the 1990's. In S. Mills (Ed.), Language and Gender. Interdisciplinary Perspectives. (pp. 31-34). London: Longman.

Cameron, D., Frazer, E., Harvey, P., Rampton, B., and Richardson, K. (1992). Researching Language. Issues of Power and Method. London: Routledge.

Cameron, L., and Larsen-Freeman, D. (2007). Complex systems and applied linguistics. International Journal of Applied Linguistics, 17(2), 226-240.

Candlin, C. N. (1987). Explaining moments of conflict in discourse. In R. Steele and T. Threadgold (Eds.), Language Topics. Essays in Honour of Michael Halliday. (pp. 413-430). Amsterdam/Philadelphia: John Benjamins.

Carney, T. (1993). "Miscommunication" and Problematic Talk. [Review]. Canadian Journal of Communication [Online], 18(1), Retrieved 15 Jan 2010: URL: http://www.cjc-online.ca/index.php/journal/article/view/2722/2628.

Carroll, K., Iedema, R., and Kerridge, R. (2008). Reshaping ICU Ward Round Practices Using Video-Reflexive Ethnography. Qualitative Health Research, 18, 380-390.

Chan, A. (2007). Same context, different strategies: a company director's discourse in business meetings Journal of Asian Pacific Communication, 17(1), 61-81. 
Chiles, T. (2006). Constructing Professional Identity: Discourse and Mentoring in the Workplace. Unpublished $\mathrm{PhD}$ Thesis, Victoria University: Wellington.

Chiles, T. (2007). The construction of an identity as 'mentor' in white collar and academic workplaces. Journal of Pragmatics, 39(4), 730-741.

Christie, F., and Martin, J. R. (Eds.). (2000). Genre and Institutions: Social Processes in the Workplace and School. London, New York: Continuum International.

Cicourel, A. V. (1992). The interpenetration of communicative contexts: examples from medical encounters. In A. Duranti and C. Goodwin (Eds.), Rethinking Context: Language as an Interactive Phenomenon (pp. 291-310). Cambridge: Cambridge University Press.

Cicourel, A. V. (2003). On contextualizing applied linguistic research in the workplace. Applied Linguistics, 24(3), 360-373.

Cicourel, A. V. (2004). Cognitive overload and communication in two healthcare settings. Communication and Medicine, 1(1), 35-43.

Cilliers, P. (2005a). Knowing complex systems. In K. Richardson (Ed.), Managing Organizational Complexity: Philosophy, Theory, and Application (pp. 7-19). Greenwich, Connecticut: Information Age Publishers.

Clyne, M. (1994). Inter-Cultural Communication at Work. Cultural Values in Discourse. Cambridge: Cambridge University Press.

Coates, J. (1987). Epistemic modality and spoken discourse. Transactions of the Philological Society, 110-131.

Coates, J. (1993). Women, Men and Language. London: Longman.

Coates, J. (Ed.). (2004). Women, Men and Language. A Sociolinguistic Account of Gender Differences in Language (3 ed.). UK: Pearson Education.

Cole, M., and Engeström, Y. (1993). A cultural-historical approach to distributed cognition. In G. Salomon (Ed.), Distributed Cognitions: Psychological and Educational Considerations (pp. 1-46). Cambridge, UK: Cambridge University Press.

Cooke, D., Brown, P., and Zhu, Y. (2007). Beyond language: workplace communication and the L2 worker. Journal of Asian Pacific Communication, 17(1), 83-103.

Cook-Gumperz, J. (2001). Cooperation, collaboration and pleasure in work. In A. di Luzio, S. Gunthner and F. Orletti (Eds.), Culture in Communication: Analyses of Intercultural Situations (pp. 117-139). Amsterdam: John Benjamins.

Cosby, K. S., and Croskerry, P. (2004). Profiles in patient safety: authority gradients in medical error. Academic Emergency Medicine, 11(12), 1341-1345.

Coupland, N. (2001). Introduction: Sociolinguistic theory and social theory. In N. Coupland, S. Sarangi and C. N. Candlin (Eds.), Sociolinguistics and Social Theory (pp. 1-26). Harlow, England: Pearson Education.

Coupland, N., Giles, H., and Wiemann, J. M. (Eds.). (1991). "Miscommunication" and Problematic Talk. London: Sage Publications. 
Coupland, N., Sarangi, S., and Candlin, C. N. (Eds.). (2001). Sociolinguistics and Social Theory. Harlow, England: Pearson Education.

Coupland, N., Wiemann, J., and Giles, H. (1991). Talk as "problem" and communication as "miscommunication": an integrative analysis. In N. Coupland, H. Giles and J. M. Wiemann (Eds.), "Miscommunication" and Problematic Talk (pp. 1-17). London: Sage Publications.

Crawford, M. (1995). Talking Difference: On Gender and Language. London and Thousand Oaks: Sage Press.

Crotty, M. (1998). The Foundations of Social Research: Meaning and Perspective in the Research Process: Sage Publications.

Cushing, S. (1994). Fatal Words: Communication Clashes and Aircraft Crashes. Chicago: University of Chicago Press.

Daly, N., Holmes, J., Newton, J., and Stubbe, M. (2004). Expletives as solidarity signals in FTAs on the factory floor. Journal of Pragmatics, 36(5), 945-964.

Dascal, M. (1999). Introduction: Some questions about misunderstanding. Journal of Pragmatics, 31, 753-762.

De Bres, J. (2009). 'Language in the Workplace Project' and 'Workplace Communication for Skilled Migrants' course at Victoria University of Wellington, New Zealand. Language Teaching, 42(04), 519-524.

Deetz, S. (1982). Critical interpretive research in organizational communication. The Western Journal of Speech Communication, 46, 131-149.

Dew, K., Dowell, A., Stubbe, M., Plumridge, E., and Macdonald, L. (2008). 'Treating' patients differently: a qualitative study of how clinical and social factors shape interactions between doctors and patients. New Zealand Family Physician, 35(6), 382-386.

Dew, K., Plumridge, E., Stubbe, M., Dowell, T., Macdonald, L., and Major, G. (2008). 'You just got to eat healthy': The topic of CAM in the general practice consultation. Health Sociology Review, 17(4), 396-409.

Dew, K., Stubbe, M., Macdonald, L., Dowell, A., and Plumridge, E. (2010). The (non)use of prioritisation protocols by surgeons. Sociology of Health and Illness, 32(4), 1-18.

Dew, K., Stubbe, M., Macdonald, L., Plumridge, E., and Dowell, A. (2007). Patient-initiated side effects talk: interactional dilemmas in the general practice consultation. Paper presented at the COMET 2007 Fifth Interdisciplinary Conference, Communication, Medicine and Ethics.

DiMatteo, R. M., Robinson, J. D., Heritage, J., Tabbarah, M., and Fox, S. A. (2003). Correspondence among patients' self-reports, chart records, and audio/videotapes of medical visits. Health Communication, 15(4), 393-413.

Dowell, A., Macdonald, L., Stubbe, M., Plumridge, E., and Dew, K. (2007). Clinicians at work: What can we learn from interactions in the consultation? New Zealand Family Physician, 34(5), 345- 350.

Drew, P. (1992). Contested evidence in courtroom cross-examination: the case of a trial for rape. In P. Drew and J. Heritage (Eds.), Talk at Work. Interaction in Institutional Settings (pp. 470-520). Cambridge: Cambridge University Press. 
Drew, P., Chatwin, J., and Collins, S. (2001). Conversation analysis: a method for research into interactions between patients and health-care professionals. Health Expectations, 4, 58-70.

Drew, P., and Heritage, J. (Eds.). (1992a). Talk at Work. Interaction in Institutional Settings. Cambridge: Cambridge University Press.

Drew, P., and Heritage, J. (1992b). Analyzing talk at work: an introduction. In P. Drew and J. Heritage (Eds.), Talk at Work. Interaction in Institutional Settings. Cambridge: Cambridge University Press.

Dunsford, J. (2009). Structured communication: improving patient safety with SBAR. Nursing for Women's Health, 13(5), 384-390.

Dwyer, J. (1990). The Business Communication Handbook (2ed ed.). Newcastle, NSW: MBC Managing Business Communication.

Dwyer, J. (1993). The Business Communication Handbook (3ed ed.). Sydney: Prentice Hall.

Eckert, P., and McConnell-Ginet, S. (1992). Think practically and look locally: language and gender as community-based practice. Annual Review of Anthropology, 21, 461-490.

Eckert, P., and McConnell-Ginet, S. (2003). Language and Gender. Cambridge: Cambridge University Press.

Edwards, D. (2004). Discursive psychology. In K. L. Fitch and R. E. Sanders (Eds.), Handbook of Language and Social Interaction (pp. 257-273). Mahwah, NJ: Lawrence Erlbaum.

Edwards, D., and Potter, J. (2005). Discursive psychology, mental states and descriptions. In H. te Molder and J. Potter (Eds.), Conversation and Cognition (pp. 241-259). Cambridge: Cambridge University Press.

Eelen, G. (2001). A Critique of Politeness Theories. Manchester: St. Jerome.

Eisenberg, E. M., and Phillips, S. R. (1991). Miscommunication in organizations. In N. Coupland, H. Giles and J. M. Wiemann (Eds.), "Miscommunication" and Problematic Talk (pp. 244-258). London: Sage.

Engeström, Y. (1992). Interactive Expertise: Studies in Distributed Working Intelligence. Helsinki: Department of Education, Helsinki University.

Engeström, Y. (1993). Developmental studies of work as a testbench of activity theory: The case of primary care medical practice. In S. Chaiklin and L. Lave (Eds.), Understanding Practice: Perspectives on Activity and Context (pp. 63103). Cambridge: Cambridge University Press.

Engeström, Y., Engeström, R., and Kerosuo, H. (2003). The discursive construction of collaborative care. Applied Linguistics, 24(3), 286-315.

Erickson, F. (1999). Appropriation of voice and presentation of self as a fellow physician: Aspects of a discourse of apprenticeship in medicine. In S. Sarangi and C. Roberts (Eds.), Talk, Work, and Institutional Order: Discourse in Medical, Mediation, and Management Settings (pp. 109-144). Berlin: Mouton de Gruyter. 
Erickson, F. (2001). Co-membership and wiggle room: some implications of the study of talk for the development of social theory. In N. Coupland, S. Sarangi and C. N. Candlin (Eds.), Sociolinguistics and Social Theory (pp. 152-181). Harlow: Longman.

Evans, S. (2007). Silence kills. Challenging unsafe practice. Kai Tiaki Nursing New Zealand, 13(3), 16-19.

Fairclough, N. (1989). Language and Power. London: Longman.

Fairclough, N. (1992a). Discourses and Social Change. Cambridge: Polity Press.

Fairclough, N. (1992b). Critical Language Awareness. London: Longman.

Fairclough, N. (1993). Critical discourse analysis and the marketization of public discourse: the universities. Discourse and Society, 4(2), 133-168.

Fairclough, N. (1995). Critical Discourse Analysis: Papers in the Critical Study of Language. London: Longman.

Fairclough, N., and Wodak, R. (1996). Critical discourse analysis. An overview. In T. A. van Dijk (Ed.), Discourse Analysis. London: Sage.

Fillary, R. (1998). Language in the workplace for students with intellectual disabilities: Research methodology issues. Te Reo: Special Issue: Proceedings of the Sixth Language and Society Conference June 1998, 41, 203-207.

Fillieatz, L. (2009). Researching workplace learning from a linguistic perspective: Power and miscommunication in the Swiss VET system. Unpublished seminar presentation, 16 November 2009. Victoria University of Wellington.

Firth, A. (Ed.). (1995). The Discourse of Negotiation: Studies of Language in the Workplace. Oxford: Pergamon Press.

Fletcher, J. M. (1999). Disappearing Acts: Gender, Power and Relational Practice at Work. Cambridge, Massachusetts: MIT Press.

Fletcher, J. R. (2003). The role of email 'conversations' in organizational knowledge creation. Paper presented at the ANZCA03 Conference, July 2003.

Foucault, M. (1982). Power/Knowledge: Selected Interviews and Other Writings by Michel Foucault, 1972-1977. New York: Pantheon.

Fraser, B. (1990). Perspectives on politeness. Journal of Pragmatics, 14(2), 219236.

Fraser, B. (1993). No conversation without misrepresentation. In H. Parret (Ed.), Pretending to Communicate (pp. 143-153). Berlin: de Gruyter.

Freed, A. F. (1992). We understand perfectly: A critique of Tannen's view of cross-sex communication. In K. Hall, M. Bucholtz and B. Moonwomon (Eds.), Locating Power: Proceedings of the Second Berkeley Women and Language Conference (Vol. 1, pp. 144-152). Berkeley: Berkeley Women and Language Group.

Freedman, A., and Medway, P. (Eds.). (1994). Genre and the New Rhetoric. London: Taylor and Francis. 
Gallois, C., and Giles, H. (1998). Accommodating mutual influence in intergroup encounters. In M. Palmer (Ed.), Progress in Communication Sciences 14: Mutual Influence (pp. 135-162). Stamford, CT: Ablex.

Gardner, J. M., and Jones, E. (1999). Problematic communication in the workplace: beliefs of superiors and subordinates. International Journal of Applied Linguistics, 9(2), 185-203.

Garfinkel, H. (1967). Studies in Ethnomethodology. Engleood Cliffs, NJ: Prentice Hall.

Gatenby, B., and Jones, D. (Eds.). (1995). Case Studies In Communication. Auckland: Longman Paul.

Geertz, C. (1973). The Interpretation of Cultures. New York: Basic Books.

Geertz, C. (1983). Local Knowledge. New York: Basic Books.

Giles, H., Coupland, J., and Coupland, N. (1991). Accommodation theory: Communication, context, and consequence. In Contexts of Accommodation: Developments in Applied Sociolinguistics (pp. 1-68). USA: Press Syndicate of University of Cambridge.

Giles, H., and Wiemann, J. M. (1987). Language, social comparison and power. In C. Berger and S. Chaffee (Eds.), The Handbook of Communication Science (pp. 350-384). Newbury Park, CA: Sage.

Gill, V., and Maynard, D. (2006). Explaining illness: patients' proposals and physicians' responses. In J. Heritage, P. Drew and D. Maynard (Eds.), Communication in Medical Care: Interactions Between Primary Care Physicians and Patients (pp. 115-150). Cambridge: Cambridge University Press.

Gilstrap, D. L. (2005). Strange attractors and human interaction: Leading complex organizations through the use of metaphors. Complicity: An International Journal of Complexity and Education, 2(1), 55-69.

Goffman, E. (1974). Frame Analysis: An Essay On The Organization Of Experience. New York: Harper and Row.

Gordon, R., D, and Grant, D. (2000). Change and the dynamics of power: a critical discursive analysis. In C. Combes, D. Grant, T. Keenoy and C. Oswick (Eds.), Organizational Discourse: Word-views, Work-views and World-views (pp. 7879). London: King's College, University of London.

Grant, C. B. (2003). Destabilizing Social Communication Theory. Theory Culture Society, 20(6), 95-119.

Grice, H. P. (1975). Logic and conversation. In P. Cole and J. Morgan (Eds.), Speech Acts. (Syntax and Semantics, Volume 3) (pp. 41-58). New York: Academic Press.

Grice, H. P. (1981). Further notes on logic and conversation. In P. Cole (Ed.), Radical Semantics. (Syntax and Semantics, Volume 9) (pp. 113-128). New York: Academic Press.

Grimshaw, A. D. (1980). Mishearing, misunderstandings, and other non-successes in talk: A plea for redress of speaker-orientated bias. Social Inquiry, 50, 31-74. 
Grimshaw, A. D. (1981). Language as Social Resource. Stanford, CA: Stanford University Press.

Grimshaw, A. D. (Ed.). (1990). Conflict Talk. Sociolinguistic Investigations of Arguments in Conversations. Cambridge: Cambridge University Press.

Gudykunst, W. B., Matsumoto, Y., Ting-Toomey, S., Nishida, T., Kim, K., and Heyman, S. (1996). The influence of cultural individualism-collectivism, self construals, and individual values on communication styles across cultures. Human Communication Research, 22, 510-543.

Gumperz, J. (1982a). Discourse Strategies. Cambridge: Cambridge University Press.

Gumperz, J. (1982b). Language and Social Identity. Cambridge: Cambridge University Press.

Gumperz, J. (1992). Interviewing in intercultural situations. In P. Drew and J. Heritage (Eds.), Talk at Work: Interaction in Institutional Settings (pp. 302-327). Cambridge: Cambridge University Press.

Gumperz, J. (1999). On Interactional sociolinguistic method. In S. Sarangi and C. Roberts (Eds.), Talk, Work, and Institutional Order: Discourse in Medical, Mediation, and Management Settings (pp. 453-471). Berlin/New York: Mouton de Gruyter.

Gumperz, J., and Hymes, D. (Eds.). (1972). Directions in Sociolinguistics: The Ethnography of Communication. New York: Holt, Rinehart and Winston.

Gumperz, J., Jupp, T. C., and Roberts, C. (1979). Cross-Talk: A Study of CrossCultural Communication. Southall, Middx: National Centre for Industrial Language Training.

Gumperz, J., and Roberts, C. (1991). Understanding in intercultural encounters. In J. Blommaert and J. Verschueren (Eds.), The Pragmatics of Intercultural and International Communication.

Hak, T. (1999). "Text" and "Con-text": Talk bias in studies of health care work. In S. Sarangi and C. Roberts (Eds.), Talk, Work and Institutional Order. Discourse in Medical, Mediation and Management Settings (pp. 427-452). Berlin, New York: Mouton de Gruyter.

Hallwright, S. (1992). Management in the mental health services: the manager psychiatrist dichotomy. Paper presented at the Progress in Forensic Psychiatry Conference, Auckland Feb 25-28.

Hamilton, W., and Britten, N. (2006). Patient agendas in primary care. British Medical Journal, 332(7552), 1225-1226.

Hammond, S. A. (1996). The Thin Book of Appreciative Inquiry. Plano, TX: Thin Book Publishing Company.

Harding, S. (1986). The Science Question in Feminism. Ithaca, NY: Cornell University Press.

Harris, S. (2003). Politeness and power: Making and responding to 'requests' in institutional settings. Text. Interdisciplinary Journal for the Study of Discourse, 23(1), 27-52. 
Haugh, M. (2007). The discursive challenge to politeness research: An interactional alternative Journal of Politeness Research, 3, 295-317.

Hawkins, P. (2004). A centennial tribute to Gregory Bateson 1904-1980 and his influence on the fields of organizational development and action research. Action Research, 2(4), 409-423.

Heath, C., and Hindmarsh, J. (2002). Analysing interaction: video, ethnography and situated conduct. In T. May (Ed.), Qualitative Research in Practice (pp. 99121). London: Sage.

Heath, C., and Luff, P. (2000). Technology in Action. Cambridge: Cambridge University Press.

Henley, N., and Kramarae, C. (1991). Gender, power and miscommunication. In N. Coupland, H. Giles and J. M. J.M. Wiemann (Eds.), "Miscommunication" and Problematic Talk. (pp. 18-43). London: Sage.

Hepburn, A., and Wiggins, S. (2005a). Developments in discursive psychology. Discourse and Society, 16(5), 595-601.

Hepburn, A., and Wiggins, S. (2005b). Size matters: Constructing accountable bodies in NSPCC helpline interaction. Discourse and Society, 16(5), 625-645.

Heritage, J. (1984). Garfinkel and Ethnomethodology. Cambridge: Polity Press.

Heritage, J. (2008). Conversation analysis as social theory. In B. Turner (Ed.), The New Blackwell Companion to Social Theory (pp. 300-320). Oxford: Blackwell.

Heritage, J., and Maynard, D. (Eds.). (2006). Communication in Medical Care: Interactions Between Primary Care Physicians and Patients. Cambridge: Cambridge University Press.

Heritage, J., Robinson, J., Elliott, M., Beckett, M., and Wilkes, M. (2007). Reducing patients' unmet concerns in primary care: the difference one word can make. Journal of General Internal Medicine, 22(10), 1429-1433.

Heritage, J., and Sefi, S. (1992). Dilemmas of advice: aspects of the delivery and reception of advice in interactions between health visitors and first-time mothers. In P. Drew and J. Heritage (Eds.), Talk at Work. Interaction in Institutional Settings (pp. 359-417). Cambridge: Cambridge University Press.

Heylighen, F. (2008). Complexity and self-organization. In M. Bates and M. Maack (Eds.), Encyclopedia of Library and Information Sciences. Brussels: Taylor and Francis.

Holmes, J. (2006). Gendered Talk at Work. Constructing Gender Identity through Workplace Discourse. Malden, MA: Blackwell Publishing.

Holmes, J. (2007). Monitoring organisational boundaries: diverse discourse strategies used in gatekeeping. Journal of Pragmatics, 39(11), 1993-2016.

Holmes, J., Fillary, R., and McLeod, M. (2000). Up Close and Interpersonal: Successful Social Interaction in the Workplace. Paper presented at the Conference of the Association for Supported Employment in New Zealand (ASENZ), Christchurch, 9-10 March 2000. 
Holmes, J., Fillary, R., McLeod, M., and Stubbe, M. (2000). Developing skills for successful social interaction in the workplace. New Zealand Journal of Disabilities Studies, 7, 70-86.

Holmes, J., and Marra, M. (2002). Over the edge?: Subversive humour between colleagues and friends. Humor, 15(1), 65-87.

Holmes, J., and Marra, M. (2004). Leadership and managing conflict in meetings. Pragmatics 14(4), 439-462.

Holmes, J., and Meyerhoff, M. (1999). The community of practice: Theories and methodologies in language and gender research. Language in Society. Special Issue, 28(2), 173-183.

Holmes, J., and Meyerhoff, M. (Eds.). (2003). The Handbook of Language and Gender. Oxford: Blackwell.

Holmes, J., and Stubbe, M. (2003a). Power and Politeness in the Workplace: a Sociolinguistic Analysis of Talk at Work. London: Longman/Pearson Education.

Holmes, J., and Stubbe, M. (2003b). Doing disagreement at work: A sociolinguistic approach. Australian Journal of Communication, 30(1), 53-77.

Holmes, J., and Stubbe, M. (2003c). Discourse in gendered workplaces: how do women manage it? In J. Holmes and M. Meyerhoff (Eds.), Handbook of Language and Gender (pp. 573-600). Oxford: Blackwell.

Holmes, J., and Stubbe, M. (2004). Strategic code-switching in New Zealand workplaces: scaffolding, solidarity and identity construction. In J. House and J. Rehbein (Eds.), Multilingual Communication. Amsterdam: John Benjamins.

Holmes, J., Stubbe, M., and Vine, B. (1999). Constructing professional identity: "doing power" in policy units. In S. Sarangi and C. Roberts (Eds.), Talk, Work and Institutional Order. Discourse in Medical, Mediation and Management Settings (pp. 351-385). Berlin and New York: Mouton de Gruyter.

House, J. (2000). Understanding misunderstanding: a pragmatic-discourse approach to analysing mismanaged rapport in talk across cultures. In H. SpencerOatey (Ed.), Culturally Speaking: Managing Rapport through Talk across Cultures (pp. 145-164). London: Continuum.

House, J., Kasper, G., and Ross, S. (Eds.). (2003). Misunderstanding in Social Life: Discourse Approaches to Problematic Talk. London: Longman.

House, J., and Rehbein, J. (Eds.). (2004). Multilingual Communication. Amsterdam: John Benjamins.

Howard, J. W. (2003). "Tower Am I Cleared to Land?": Pilot-ATC (Mis)Communication. Unpublished PhD, UMI, Ann Arbor, MI.

Howard, J. W. (2008). Tower, am I cleared to land?: Problematic communication in aviation discourse. Human Communication Research, 34(3), 370-391.

Hughes, M. (2005). The Voice of Prophets (Vol. 2). Morrrisville, North Carolina: Lulu.com.

Hunston, S. (Ed.). (1998). Language at Work. Selected Papers from the Annual Meeting of the British Association of Applied Linguistics. Clevedon: British Association of Applied Linguistics in association with Multilingual Matters. 
Hutchby, I., and Wooffitt, R. (1998). Conversation Analysis: Principles, Practices and Applications. Oxford, UK: Polity Press.

Hymes, D. (1974). Foundations in Sociolinguistics: An Ethnographic Approach. Philadelphia: University of Pennsylvania Press.

Iedema, R., and Scheeres, H. (2003). From doing work to talking work: Renegotiating knowing, doing and identity. Applied Linguistics, 24(3), 316-337.

Innes, A. D., Campion, P. D., and Griffiths, F. E. (2005). Complex consultations and the 'edge of chaos'. British Journal of General Practice, 55(510), 47-52.

Jacobs, S. (2002). Maintaining neutrality in dispute mediation: managing disagreement while managing not to disagree. Journal of Pragmatics, 34, 14031426.

Jaworski, A. (Ed.). (1997). Silence. Interdisciplinary Perspectives. Berlin: Mouton de Gruyter.

Jefferson, G. (1984). On the organization of laughter in talk about troubles. In J. M. Atkinson and J. Heritage (Eds.), Structures of Social Action: Studies in Conversation Analysis (pp. 346-369). Cambridge: Cambridge University Press.

Jefferson, G. (1988). On the sequential organization of troubles-talk in ordinary conversation. Social Problems, 35(4), 418-441.

Jefferson, G. (1992). The rejection of advice: managing the problematic convergence of a "troubles-telling" and a "service encounter". In P. Drew and J. Heritage (Eds.), Talk at Work: Interaction in Institutional Settings (pp. 521-548). Cambridge: Cambridge University Press.

Jones, A., and Bugge, C. (2006). Improving understanding and rigour through triangulation: an exemplar based on patient participation in interaction. Journal of Advanced Nursing, 55(5), 612-621.

Jones, D. (1998). Language in the workplace: Towards a model for evaluation and development. Te Reo: Special Issue: Proceedings of the Sixth Language and Society Conference June 1998, 41, 193-195.

Jones, D. (2001). Re-Generating the Communication Audit. Paper presented at the Australia and New Zealand Communication Association (ANZCA) Conference, Perth: Australia.

Jones, D. (2002). The interpretive auditor: Reframing the communication audit. Management Communication Quarterly, 15(3), 466-471.

Jones, D., and Stubbe, M. (2004). Communication and the reflective practitioner: a shared perspective from sociolinguistics and organisational communication International Journal of Applied Linguistics, 14(2), 185-211.

Kasper, G., and Ross, S. J. (2007). Multiple questions in oral proficiency interviews. Journal of Pragmatics, 39(11), 2045-2070.

Kell, S., Marra, M., Holmes, J., and Vine, B. (2007). Ethnic differences in the dynamics of women's work meetings. Mulitilingua, 26, 309-331.

Kendall, S., and Tannen, D. (1997). Gender and language in the workplace. In R. Wodak (Ed.), Gender and Discourse (pp. 81-105). London: Sage. 
Kerekes, J. (2007a). Introduction to the special issue "High stakes gatekeeping encounters and their consequences: Discourses in intercultural institutional settings". Journal of Pragmatics, 39(11), 1891-1894.

Kerekes, J. (2007b). The co-construction of a gatekeeping encounter: An inventory of verbal actions. Journal of Pragmatics, 39(11), 1942-1973.

King, J. (1999). Talking bro: Maori English in the university setting. Te Reo, 42, 20-38.

Kitzinger, C. (2005a). Sexual harassment: a discursive approach. In S. Wilkinson and C. Kitzinger (Eds.), Feminism and Discourse: Psychological Perspectives (pp. 32-48). London: Sage.

Kitzinger, C. (2005b). Heteronormativity in action: reproducing normative heterosexuality in 'after hours' calls to the doctor. Social Problems, 52, 477-498.

Kreitner, R., and Kinicki, A. (1992). Organizational Behaviour (2 ed.). Boston: Irwin.

Kress, G. (1990). Critical discourse analysis. Annual Review of Applied Linguistics, 11, 84-99.

Kress, G., and Hodge, R. (1979). Language as Ideology. London: Routledge and Kegan Paul.

Ladegaard, H., J. (2009). Pragmatic cooperation revisited: Resistance and noncooperation as a discursive strategy in asymmetrical discourses. Journal of Pragmatics, 41(4), 649-666.

Lakoff, G., and Johnson, M. (1980). Metaphors We Live By. Chicago: University of Chicago Press.

Lane, C., and Hilder, J. (2003). Multiple discourse analyses of a workplace interaction [Conversation Analysis Section]. Discourse Studies, 5(3), 351-388.

Larsen-Freeman, D., and Cameron, L. (2008). Complex Systems and Applied Linguistics. Oxford: Oxford University Press.

Lave, J., and Wenger, E. (1991). Situated Learning: Legitimate Peripheral Participation. Cambridge, UK: Cambridge University Press.

Lee, D. (1992). Competing Discourses: Perspective and Ideology in Language. London, New York: Longman.

Leech, G. N. (1983). Principles of Pragmatics. London: Longman.

Levinson, S. (1983). Pragmatics. Cambridge: Cambridge University Press.

Levinson, S. (2005). Living with Manny's dangerous idea. Discourse Studies, 7(4), 431-453.

Linell, P. (1995). Troubles with mutualities: a dialogical theory of misunderstanding and miscommunication. In I. Markovà, C. F. Graumann and K. Foppa (Eds.), Mutualities in Dialogue (pp. 176-213). Cambridge: Cambridge University Press. 
Lingard, L., Espin, S., Rubin, B., Whyte, S., Colmenares, M., Baker, G. R., Doran, D., Grober, E., Orser, B., Bohnen, J., and Reznick, R. (2005). Getting teams to talk: development and pilot implementation of a checklist to promote interprofessional communication in the OR. Quality and Safety in Health Care, 14(5), 340-346.

Lingard, L., Espin, S., Whyte, S., Regehr, G., Baker, G. R., Reznick, R., Bohnen, J., Orser, B., Doran, D., and Grober, E. (2004). Communication failures in the operating room: an observational classification of recurrent types and effects. Quality and Safety in Health Care, 13(5), 330-334.

Lingard, L., Schryer, C. F., Spafford, M. M., and Garwood, K. (2003). Talking the talk: School and workplace genre tension in clerkship case presentations. Medical Education, 37, 612-620.

Littlejohn, S. W. (2001). Theories of Communication (7th ed.). Belmont, CA: Wadsworth.

Locher, M. A., and Watts, R. J. (2005). Politeness theory and relational work. Journal of Politeness Research, 1(1), 9-34.

LWP. (2003). Language in the Workplace Project: Homepage. School of Linguistics and Applied Language Studies, Victoria University of Wellington. URL: http://www.vuw.ac.nz/lals/lwp.

Macdonald, L. (2002). Nurse Talk. Unpublished MA (Applied) Thesis, Victoria University of Wellington, Wellington.

Mackiewicz, J., and Riley, K. (2002). Balancing clarity and politeness in editing sessions with non-native speakers. In Proceedings of IPCC 2002: Reflections on Communication. Paper presented at the IEEE International Professional Communication Conference (IPCC 2002) SEP 17-20, 2002, Portland, Oregon.

Major, G., and Holmes, J. (2008). How do nurses describe health care procedures? Analysing nurse-patient interaction in a hospital ward. Australian Journal of Advanced Nursing Studies, 25(4), 58-70.

Maltz, D. N., and Borker, R. A. (1982). A cultural approach to male-female miscommunication. In J. J. Gumperz (Ed.), Language and Social Identity (pp. 196-216). Cambridge: Cambridge University Press.

Marra, M. (2003). Decisions in New Zealand business meetings: A sociolinguistic analysis of power at work. Unpublished $\mathrm{PhD}$ thesis,, Victoria University of Wellington, Wellington, New Zealand.

Marshall, S., Harrison, J., and Flanagan, B. (2008). The evaluation of structured communication tools in healthcare. Human Factors and Ergonomics Society Annual Meeting Proceedings, 52(12), 860-864.

Maxfield, D., Grenny, J., McMillan, R., Patterson, K., and Switzler, A. (2005). Silence Kills. The Seven Crucial Conversations for Healthcare. American Association of Critical Care Nurses. Retrieved 5/02/2010.

URL: www.aacn.org/aacn/pubpolicy.nsf/Files/SilenceKills

McKeon, L. M., Oswaks, J. D., and Cunningham, P. D. (2006). Safeguarding patients: complexity science, high reliability organizations, and implications for team training in healthcare. Clinical Nurse Specialist, 20(6), 298-304. 
McTear, M. F., and King, F. (1991). Miscommunication in clinical contexts: the speech therapy interview. In N. Coupland, H. Giles and J. M. Wiemann (Eds.), "Miscommunication" and problematic talk (pp. 195-214). London: Sage Publications.

Metge, J., and Kinloch, P. (1978). Talking Past Each Other: Problems of CrossCultural Communication. Wellington: Victoria University of Wellington / Price Milburn.

Mills, S. (2002). Rethinking politeness, impoliteness and gender identity. In L. Liteselliti and J. Sunderland (Eds.), Discourse Analysis and Gender Identity (pp. 69-90). Amsterdam: John Benjamins.

Milroy, L. (1984). Comprehension and context: Successful communication and communicative breakdown. In P. Trudgill (Ed.), Applied Sociolinguistics (pp. 731). London: Academic Press.

Moerman, M. (1988). Talking Culture, Ethnography and Conversation Analysis. Philadelphia: University of Pennsylvania Press.

Morand, D. A. (1996a). Dominance, deference, and egalitarianism in organisational interaction: A sociolinguistic analysis of power and politeness. Organizational Science, 7(5), 544-556.

Morand, D. A. (1996b). Politeness as a universal variable in cross-cultural managerial communication. International Journal of Organizational Analysis, 4(1), 52-74.

Morgan, S. (2008). Miscommunication in General Practice Consultations: a Microanalysis of Communication Mismatches in General Medical Consultations. Unpublished MHSc Thesis, Otago University, Wellington.

Moss, B., and Roberts, C. (2005). Explanations, explanations, explanations: how do patients with limited English construct narrative accounts in multi-lingual, multi-ethnic settings, and how can GPs interpret them? Family Practice, 22(4), 412-418.

Mullany, L. (2006). "Girls on tour": Politeness, small talk, and gender in managerial business meetings. Journal of Politeness Research, 2(1), 55-77.

Mumby, D. K., and Clair, R. P. (1997). Organizational discourse. In T. A. Van Dijk (Ed.), Discourse Studies: A Multidisciplinary Introduction (Vol. 2, pp. 181205). London: Sage.

Murphy, V. (2009). Review of 'Complex Systems and Applied Linguistics', Diane Larsen-Freeman, Lynne Cameron, Oxford University Press, Oxford (2008). System, 37, 343-344.

Musson, G., and Cohen, L. (1999). Understanding language processes: A neglected skill in the management curriculum. Management Learning, 30(1), 2742.

Neill, D. (1996). Cross-Cultural Communication: Collaboration in Intercultural Discourse: Examples from a Multicultural Workplace. Frankfurt am Main: Peter Language.

Nevile, M. (2004). Beyond the Black Box: Talk-in-interaction in the Airline Cockpit. Aldershot: Ashgate. 
Ng, S. H., and Bradac, J. J. (1993). Power in Language. Verbal Communication and Social Influence (Vol. 3). London: Sage.

OED-Online. (2010). Oxford English Dictionary. Retrieved 22/3/2010, URL: http://dictionary.oed.com

Olsina, E. C. (2002). "Managing understanding in intercultural talk: an empirical approach to miscommunication.". Atlantis, revista de la Asociación Española de Estudios Anglo-Norteamericanos 24(1).

Orlikowski, W., and Yates, J. (1994). Genre repertoire: The structuring of communication practices in organizations. Administrative Science Quarterly, 39(4), 541-574.

Pateman, T. (1980). Language, Truth and Politics: Towards a Radical Theory for Communication. Sussex: Jean Stroud.

Pearce, W. B. (1994). Interpersonal Communication: Making Social Worlds. New York: Harper Collins College Publishers.

Perkins, M. R. (1998). Is pragmatics epiphenomenal? Evidence from communication disorders. Journal of Pragmatics, 29(3), 291-311.

Pilnick, A. (2002). 'There are no rights and wrongs in these situations': identifying interactional difficulties in genetic counselling. Sociology of Health and Illness, 24(1), 66-88.

Pilnick, A., Hindmarsh, J., and Gill, V. T. (Eds.). (2010). Communication in Health Care Settings. Policy, Participation and New Technologies. UK: WileyBlackwell.

Potter, J. (2005). Making psychology relevant. Discourse and Society, 16(5), 739747.

Potter, J., and Hepburn, A. (2003). "I'm a bit concerned" - call openings on a child protection helpline. Research on Language and Social Interaction 36, 197-240.

Putnam, L. L. (2000). Word-views and work-views: Building theory about discourse and organizations. In C. Combes, D. Grant, T. Keenoy and C. Oswick (Eds.), Proceedings of Conference on Organizational Discourse: Word-views, Work-views and World-views. King's College, University of London, 26-28 July 2000 (pp. 225). London: KMPC.

Putnam, L. L., and Fairhurst, G. K. (2001). Discourse analysis in organizations. Issues and concerns. In F. M. Jablin and L. L. Putnam (Eds.), The New Handbook of Organizational Communication. Advances in Theory, Research and Methods (2 ed., pp. 78-136). Thousand Oaks, California/London: Sage Publications.

Raymond, G., and Heritage , J. (2006). The epistemics of social relations: Owning grandchildren. Language in Society, 35, 677-705.

Reason, J. (2000). Human error: models and management. British Medical Journal, 320, 768-770.

Reason, J. (2004). Beyond the organisational accident: the need for "error wisdom" on the frontline. Quality and Safety in Health Care, 13(Suppl II), ii28-ii33.

Redding, W. C. (1972). Communication within the Organization. New York: Industrial Communication Council. 
Richardson, K. (2008). Managing complex organizations: complexity thinking and the science and art of management. E:CO Emergence: Complexity and Organization 10(2), 13-26.

Richardson, K. (Ed.). (2005). Managing Organizational Complexity: Philosophy, Theory, and Application. Greenwich, Connecticut: Information Age Publishers.

Richardson, K., van Uden, J., and Cilliers, P. (2000). Complexity science as epistemology. In C. Combes, D. Grant, T. Keenoy and C. Oswick (Eds.), Proceedings of Conference on Organizational Discourse: Word-views, Workviews and World-views. King's College, University of London, 26-28 July 2000 (pp. 232-234). London: KMPC.

Riley, S. C. E. (2002). Constructions of equality and discrimination in professional men's talk. British Journal of Social Psychology, 41, 443-461.

Roberts, C. (1997). "There's nothing so practical as some good theories". International Journal of Applied Linguistics, 7(1), 66-78.

Roberts, C. (2001). 'Critical' social theory: Good to think with or something more? In N. Coupland, S. Sarangi and C. N. Candlin (Eds.), Sociolinguistics and Social Theory (pp. 1-26). Harlow, England: Pearson Education.

Roberts, C. (2009). 'Mince' or 'mice'? Clinical miscommunication and patient safety in a linguistically diverse community. In B. Hurwitz and S. Aziz (Eds.), Health Care Errors and Patient Safety (pp. 112-128). Oxford: Blackwell Publishing Ltd.

Roberts, C., Davies, E., and Jupp, T. (1992). Language and Discrimination: A Study of Communication in Inter-Ethnic Workplaces. London: Longman.

Roberts, C., Moss, B., Wass, V., Sarangi, S., and Jones, R. (2005). Misunderstandings: a qualitative study of primary care consultations in multilingual settings, and educational implications. Medical Education, 39, 465475.

Roberts, C., and Sarangi, S. (1999). Hybridity in gatekeeping discourse: Issues of practical relevance for the researcher. In S. Sarangi and C. Roberts (Eds.), Talk, Work and Institutional Order Discourse in Medical, Mediation and Management Settings (pp. 473-503). Berlin and New York: Mouton de Gruyter.

Roberts, C., and Sarangi, S. (2003). Uptake of discourse research in interprofessional settings: reporting from medical consultancy. Applied Linguistics, 24(3), 338-359.

Roberts, C., and Sarangi, S. (2005). Theme-oriented discourse analysis of medical encounters. Medical Education, 39(6), 632-640.

Roberts, C., Sarangi, S., and Moss, B. (2004). Presentation of self and symptoms in primary care consultations involving patients from non-English speaking backgrounds. Communication and Medicine, 1(2), 159-169.

Roberts, C., Sarangi, S., Southgate, L., Wakeford, R., Wass, V., Esmail, A., and May, C. (2000). Oral examinations: equal opportunities, ethnicity, and fairness in the MRCGP. British Medical Journal, 320(7231), 370-375. 
Roberts, C., and Sayers, P. (1987). Keeping the gate: How judgments are made in interethnic interviews. In K. Knapp, W. Enniger and A. Knapp-Potthoff (Eds.), Analysing Intercultural Communication (pp. 111-136). Berlin: Mouton de Gruyter.

Roberts, M. (1999). Lever Rexona: Report on ESOL Review 1999. Wellington: LWP, Victoria University of Wellington.

Rouveyrol, L., Maury-Rouan, C., Vion, R., and Marie-Christine, N.-J. (2005). A linguistic toolbox for discourse analysis: towards a multidimensional handling of verbal interactions Discourse Studies, 7(3), 289-313.

Sacks, H. (1967). The search for help: no one to turn to. In E. S. Shneidman (Ed.), Essays in Selfdestruction (pp. 203-223). New York: Science House.

Sacks, H. (1972a). An initial investigation of the usability of conversational data for doing sociology. In D. Sudnow (Ed.), Studies in Social Interaction (pp. 3174). New York: Free Press.

Sacks, H. (1972b). On the analyzability of stories by children. In J. J. Gumperz and D. Hymes (Eds.), Directions in Sociolinguistics: the Ethnography of Communication (pp. 325-345). New York: Rhinehart and Winston.

Sacks, H. (1984). Notes on methodology. In J. M. Atkinson and J. Heritage (Eds.), Structures of Social Action: Studies in Conversation Analysis (pp. 21-27). Cambridge: Cambridge University Press.

Sacks, H., Schegloff, E. A., and Jefferson, G. (1974). A simplest systematics for the organization of turn taking for conversation. Language, 50, 696-735.

Salas, E., Wilson, K. A., Murphy, C. E., King, H., and Salisbury, M. (2008). Communicating, coordinating, and cooperating when lives depend on it: tips for teamwork. Joint Commission Journal on Quality and Patient Safety, 34(6), 333341.

Sarangi, S. (2002). Discourse practitioners as a community of interprofessional practice: some insights from health communication research. In C. Candlin (Ed.), Research and Practice in Professional Discourse (pp. 95-135). Hong Kong: City University of Hong Kong Press.

Sarangi, S. (2004). Editorial: Towards a communicative mentality in medical and healthcare practice. Communication and Medicine, 1(1), 1-11.

Sarangi, S. (2005). Interactional expertise in healthcare encounters. Communication and Medicine, 2(2), 103-104.

Sarangi, S., and Roberts, C. (1999a). The dynamics of interactional and institutional orders. In S. Sarangi and C. Roberts (Eds.), Talk, Work, and Institutional Order: Discourse in Medical, Mediation, and Management Settings (pp. 1-57). Berlin: Mouton de Gruyter.

Sarangi, S., and Roberts, C. (Eds.). (1999b). Talk, Work, and Institutional Order: Discourse in Medical, Mediation, and Management Settings. Berlin: Mouton de Gruyter. 
Sarangi, S., and Roberts, C. (2002). Discoursal (mis)alignments in professional gatekeeping settings. In C. J. Kramsch (Ed.), Language Acquisition and Language Socialization. Ecological Perspectives (pp. 197-227). London, New York: Continuum.

Sarangi, S., and Slembrouk, S. (1996). Language, Bureaucracy and Social Control. London: Longman.

Saville-Troike, M. (2003). The Ethnography of Communication. An Introduction (3 ed.). Oxford: Blackwell Publishing.

Schegloff, E. A. (1968). Sequencing in conversational openings. American Anthropologist, 70, 1075-1095.

Schegloff, E. A. (1972). Notes on a conversational practice: formulating place. In D. Sudnow (Ed.), Studies in Social Interaction (pp. 75-119). New York: Free Press.

Schegloff, E. A. (1984). On some questions and ambiguities in conversation. In J. M. Atkinson and J. Heritage (Eds.), Structures of Social Action (pp. 29-52). Cambridge: Cambridge University Press.

Schegloff, E. A. (1987). Some sources of misunderstanding in talk-in-interaction. Linguistics, 25, 201-218.

Schegloff, E. A. (1997). Whose text? Whose context? Discourse and Society, 8(2), 1651-1687.

Schick Case, S. (1995). Gender, language and the professions: recognition of wide-verbal-repertoire speech. Studies in the Linguistic Sciences, 252(Fall 1995), 150-192.

Schiffrin, D. (1994). Approaches to Discourse. Cambridge: Blackwell Publishers Ltd.

Schnurr, S. (2009). Constructing leader identities through teasing at work. Journal of Pragmatics, 41, 1125-1138.

Schnurr, S., and Chan, A. (2009). Leadership discourse and politeness at work. A cross-cultural case study of New Zealand and Hong Kong. Journal of Politeness Research, 5(2), 131-157.

Schnurr, S., Marra, M., and Holmes, J. (2007). Being (im)polite in New Zealand workplaces: Maori and Pakeha leaders. Journal of Pragmatics, 39(4), 712-729.

Schryer, C. F., Gladkova, O., Spafford, M. M., and Lingard, L. (2007). Comanagement in healthcare: negotiating professional boundaries. Discourse and Communication, 1(4), 452-479.

Schryer, C. F., Lingard, L., Spafford, M., and Garwood, K. (2003). Structure and agency in medical case presentations. In C. Bazerman and D. Russell (Eds.), Writing Selves, Writing Societies. Research from Activity Perspectives (pp. 6296). Colorado State University, Fort Collins The WAC Clearinghouse http://wac.colostate.edu.

Scollon, R., and Wong-Scollon, S. (2001). Intercultural Communication: A Discourse Approach (2 ed.). Oxford: Blackwell. 
Silverman, D. (1999). Warriors or collaborators: Reworking methodological controversies in the study of institutional interaction. In S. Sarangi and C. Roberts (Eds.), Talk, Work and Institutional Order. Discourse in Medical, Mediation and Management Settings (pp. 401-452). Berlin, New York: Mouton de Gruyter.

Silverman, D. (2001). Interpreting Qualitative Data: Methods for Analyzing Talk, Text and Interaction (2 ed.). London: Sage publications.

Sligo, F. X., and Bathurst, R. J. (2005). Communication in the New Zealand Workplace: Theory and Practice. NZ: Software Technology NZ, Limited.

Smart, G. (2003). A central bank's “communications strategy": The interplay of activity, discourse genres, and technology in a time of organizational change. In C. Bazerman and D. Russell (Eds.), Writing Selves, Writing Societies. Research from Activity Perspectives (pp. 9-61). Colorado State University, Fort Collins: The WAC Clearinghouse http://wac.colostate.edu.

Sollitt-Morris, L. (1996). Language, Gender and Power Relationships: the Enactment of Repressive Discourse in Staff Meetings of Two Subject Departments in a New Zealand Secondary School. Unpublished Ph.D. Thesis, Victoria University of Wellington, Wellington.

Spencer-Oatey, H., and Jiang, W. (2002). Explaining cross-cultural pragmatic findings: moving from politeness maxims to sociopragmatic interactional principles (SIPs). Journal of Pragmatics, 35, 1633-1650.

Sperber, D., and Wilson, D. (1985). Relevance: Communication and Cognition. Oxford: Blackwell.

Stahl, K., Palileo, A., Schulman, C. I., Wilson, K., Augenstein, J., Kiffin, C., and McKenney, M. (2009). Enhancing patient safety in the trauma/surgical intensive care unit. Journal of Trauma-Injury Infection and Critical Care, 67(3), 430-435.

Statistics-NZ. (2010). QuickStats. About Culture and Identity. 2006 Census. Retrieved 21/1/2010. URL: http://www.stats.govt.nz/Census/2006CensusHomePage.aspx

Stein, N. L., and Bernas, R. S. (1997). Conflict talk: Understanding and resolving arguments. In T. Givon (Ed.), Conversation: Cognitive, Communicative and Social Perspectives (pp. 233-268). Amsterdam: John Benjamins.

Stevenson, F., Cox, K., Britten, N., and Dundar, Y. (2004). A systematic review of the research on communication between patients and health care professionals about medicines: the consequences for concordance. Health Expectations, 7, 235245.

Stewart, J., D'Angelo, G., and Logan, C. (1997). Together. Communicating Interpersonally (5th Revised ed.). USA: McGraw-Hill.

Stivers, T. (2005). Parent resistance to physicians' treatment recommendations: one resource for initiating a negotiation of the treatment decision. Health Communication, 18(1), 41-74.

Stivers, T., and Majid, A. (2007). Questioning children: interactional evidence on implicit bias in medical interviews. Social Psychology Quarterly, 70(4), 424-441. 
Stubbe, M. (1997a). Collaborating through talk in professional workplaces: patterns of discourse organisation in problem-solving discussions. Paper presented at the Twelfth New Zealand Linguistic Society Conference: Dunedin, November 1997.

Stubbe, M. (1997b). Intergroup Communication in Professional Contexts: a Literature Review. Unpublished report prepared for CRESA (Centre for Research, Evaluation and Social Assessment): Wellington.

Stubbe, M. (1998a). Researching language in the workplace: a participatory model. Paper presented at the Australian Linguistics Society Conference, July 1998, Brisbane University of Queensland July 1998. http://english.uq.edu.au/linguistics/als/als98/.

Stubbe, M. (1998b). Striking a balance: Language, gender and professional identity. In S. Wertheim, A. C. Bailey and M. Corston-Oliver (Eds.), Proceedings of the Fifth Berkeley Women and Language Conference (pp. 545-556). Berkeley, California: Berkeley Women and Language Group.

Stubbe, M. (1998c). Are you listening? Cultural influences on the use of supportive verbal feedback in conversation. Journal of Pragmatics, 29, 257-289.

Stubbe, M. (1998d). Effective communication in the workplace: patterns of discourse organisation in task-oriented discussions. Paper presented at the Sixth CLESOL Conference, Palmerston North 1998.

Stubbe, M. (1999). Just joking and playing silly buggers: Humour and teambuilding on a factory production line. Paper presented at the NZ Linguistics Society Conference, Massey University, 24-26 November 1999.

Stubbe, M. (2000a). "Just do it...!": Discourse strategies for 'getting the message across' in a factory production team. In J. Henderson (Ed.), Proceedings of the 1999 Conference of the Australian Linguistic Society, University of Western Australia, 28 September - 2 October, 1999. Perth: Retrieved October 2000. URL: http://www.arts.uwa.edu.au/LingWWW/als99/proceedings

Stubbe, M. (2000b). Talk that works: evaluating communication in a factory production team. New Zealand English Journal, 14.

Stubbe, M. (2000c). What is miscommunication anyway? Analysing problematic discourse in workplace interactions. In C. Combes, D. Grant, T. Keenoy and C. Oswick (Eds.), Proceedings of Conference on Organizational Discourse: Wordviews, Work-views and World-views. King's College, University of London, 2628 July 2000 (pp. 265-267). London: KMPC.

Stubbe, M. (2001). From office to production line: collecting data for the Wellington Language in the Workplace Project, Language in the Workplace Occasional Papers (Vol. 2). Wellington: Victoria University of Wellington, URL: http://www.victoria.ac.nz/lals/lwp/resources/occasional-papers.aspx.

Stubbe, M. (2002). Effective workplace talk: Developing effective inter-personal communication in the workplace (Workshop). Paper presented at the International Conference on Language and Social Psychology (ICLASP) 8. Hong Kong July 14-19, 2002. 
Stubbe, M. (2004). A fly on the wall or a fly in the ointment? Analysing interaction in general practice consultations. Paper presented at the 9th NZ Language and Society Conference, Massey University, Palmerston North, August 2004.

Stubbe, M. (2009). Review of the book 'Variational Pragmatics: A Focus on Regional Varieties in Pluricentric Languages'. Journal of Politeness Research, 5(2009), 317-323.

Stubbe, M., and Brown, P. (2002). Talk that works. Communication in successful factory teams: A training resource kit.[Video and handbook]. Wellington: School of Linguistics and Applied Language Studies, Victoria University of Wellington.

Stubbe, M., and Dew, K. (2009). Shall I say about 60?" The (re)construction and reification of diagnostic information in health encounters. Paper presented at the International Pragmatics Association Conference (IPRA), 13- 17 July 2009, Melbourne, Australia.

Stubbe, M., Dew, K., Dowell, A., Macdonald, L., and Plumridge, E. (2006). "You'll be persuaded?" The discursive construction of referral decisions in general practice consultations. Paper presented at the 10th New Zealand Language and Society Conference, August 2006, Christchurch, New Zealand.

Stubbe, M., Dew, K., Dowell, A., Vernall, S., Moriarty, H., and Morgan, S. (forthcoming). From talk to text: encoding medical notes in the general practice consultation.

Stubbe, M., Dew, K., Macdonald, L., and Dowell, A. (2009). Tracking patientprofessional interactions through an episode of care: What are the implications for practice? . Paper presented at the Myths and Realities of Primary Care: RNZCGP Annual Scientific Conference, Wellington: 9-12 September 2009. Retrieved November 2009

URL:http://www.conference.co.nz/files/RNZCGP\%202009\%20_Stubbe-ARCH\%20FINAL.pdf

Stubbe, M., Dowell, A., Plumridge, E., Macdonald, L., and Dew, K. (2008). Antibiotics prescribing dilemmas: Do our GPs say one thing while doing another? . New Zealand Pharmacy Journal, May 2008, Page 2028ff. Retrieved January 2009. URL: http://www.pharmacyjournal.co.nz/show_article.php?id=30362.

Stubbe, M., and Holmes, J. (1995). You know, eh and other "exasperating expressions": An analysis of social and stylistic variation in the use of pragmatic devices in a sample of New Zealand English. Language and Communication, 15(1), 63-88.

Stubbe, M., and Holmes, J. (2000). Talking Maori or Pakeha in English: Signalling identity in discourse. In A. Bell and K. Kuiper (Eds.), New Zealand English (pp. 249-278). Amsterdam/Wellington: John Benjamins/Victoria University Press.

Stubbe, M., Holmes, J., Vine, B., and Marra, M. (2001). Forget Mars and Venus, let's get back to earth: Challenging gender stereotypes in the workplace. In J. Holmes (Ed.), Gendered Speech in Social Context: Perspectives from Gown and Town. Wellington: Victoria University Press.

Stubbe, M., and Ingle, M. (1999). Collecting natural interaction data in a factory: Some methodological challenges. Paper presented at the Murdoch Symposium on Talk-in-Interaction: Perth, September 1999. Retrieved August 2008.

URL: http://www.vuw.ac.nz/lals/lwp/resources/stubbe_and_ingle_1999.htm. 
Stubbe, M., Lane, C., Hilder, J., Vine, E., Vine, B., Marra, M., Holmes, J., and Weatherall, A. (2003). Multiple discourse analyses of a workplace interaction. Discourse Studies, 5(3), 351-388.

Stubbe, M., and Marra, M. (1999). Developing Organisational Communication: Report on Pilot of Communication Evaluation and Development Process. Unpublished report prepared for Mobil Oil NZ Ltd.

Sturmberg, J. P., and Cilliers, P. (2009). Time and the consultation - an argument for a 'certain slowness'. Journal of Evaluation in Clinical Practice, 15, 881-885.

Sunaoshi, Y. (1999). Language Use in Japanese Manufacturing Plants in the United States. Unpublished $\mathrm{PhD}$ Thesis, University of Texas at Austin.

Sunaoshi, Y. (2005). Historical context and intercultural communication: Interactions between Japanese and American factory workers in the American South. Language in Society, 34(2), 185-217.

Swales, J. (2004). Research Genres: Exploration and Applications. Cambridge: Cambridge University Press

Tajfel, H. (Ed.). (1982). Social Identity and Intergroup Relations. Cambridge: Cambridge University Press.

Tajfel, H., and Turner, J. C. (1979). An integrative theory of intergroup conflict. In W. G. Austin and S. Worchel (Eds.), The Social Psychology of Intergroup Relations (pp. 33-47). Belmont: Wadsworth.

Tannen, D. (1981). The machine-gun question: an example of conversational style. Journal of Pragmatics, 5, 383-397.

Tannen, D. (1990). You Just Don't Understand. Women and Men in Conversation. Australia: Random House.

Tannen, D. (Ed.). (1993a). Framing in Discourse. New York, Oxford: Oxford University Press.

Tannen, D. (Ed.). (1993b). Gender and Conversational Interaction. Oxford and New York: Oxford University Press.

Tannen, D. (1994). Talking from 9 to 5: Women and Men in the Workplace: Language, Sex and Power. London: Virago Press.

Tannen, D. (1999). The display of (gendered) identities in talk at work. In M. Bucholtz, A. C. Liang and L. A. Sutton (Eds.), Reinventing Identities: The Gendered Self in Discourse (pp. 221-240). New York, Oxford: Oxford University Press.

Taylor, J., Flanagin, A., Cheney, G., and Siebold, D. (2001). Organizational communication research: key moments, central concerns and future challenges. Communication Yearbook, 24, 99-137.

Taylor, J. R., and Lerner, L. (1996). Making sense of sensemaking: How managers construct their organization through their talk. Studies in Cultures, Organizations and Societies, 2, 257-286.

Taylor, M. E. (1987). Functions of in-house language: Observations on data collected from some British financial institutions. Language in Society, 16(1), 1-5. 
Ten Have, P. (2007). Doing Conversation Analysis. A Practical Guide (2 ed.). London: Sage Publications.

Thomas, J. (1983). Cross-cultural pragmatic failure. Applied Linguistics, 4(2), 91112.

Thomas, J. (1995). Meaning in Interaction: An Introduction to Pragmatics. Harlow, Essex: Longman.

Thornborrow, J. (2002). Power Talk: Language and Interaction in Institutional Discourse. Harlow: Longman.

Tietze, S., Cohen, L., and Musson, G. (2003). Understanding Organizations Through Language. London: Sage Publications.

Timmermans, S., and Berg, E. (2003). The practice of medical technology. Sociology of Health and Illness, 25, 97-114.

Tourish, D., and O'Hargie, O. (2000). Auditing communication to maximise performance. In D. Tourish and O. O'Hargie (Eds.), Handbook of Communication Audits for Organisations (pp. 22-41). London: Routledge.

Tracy, K. (1997). Interactional trouble in emergency service requests: a problem of frames. Research on Language and Social Interaction, 30(4), 315-343.

Tracy, K., and Coupland, N. (Ed.). (1990). Multiple Goals in Discourse. Clevedon, UK: Multilingual Matters.

Trenholm, S. (1999). Thinking Through Communication: An Introduction to the Study of Communication (2 ed.). Boston: Allyn and Bacon.

Trenholm, S. (2010). Thinking Through Communication: An Introduction to the Study of Human Communication (6th International ed.). USA: Pearson Education Limited.

Tulin, M. F. (1997). Talking organization: Possibilities for conversation analysis in organizational behaviour research. Journal of Management Inquiry, 6(2), 101119.

Tzanne, A. (2000). Talking at Cross-purposes: the Dynamics of Miscommunication. Germany: John Benjamins Publishing Company.

Ulijn, J., O’Hare, D., Weggeman, M., Ledlow, G., and Hall, H. (2000). Innovation, corporate strategy and cultural context: What is the mission for international business communication? Journal of Business Communication, 37(3), 202-208.

Unger, R. K. (Ed.). (1989). Representations: Social Constructions of Gender. Amityville, NY: Baywood.

Urry, J. (2005). The Complexity Turn. Theory Culture Society, 22(5), 1-14.

Van Dijk, T. (1987). Handbook of Discourse Analysis (Vols 1-4). London: Academic Press.

Van Dijk, T. (1990). Social cognition and discourse. In H. H Giles and W. P. Robinson (Eds.), Handbook of Social Psychology (pp. 163-186). New York: John Wiley and Sons. 
Van Dijk, T. A. (1998). Principles of critical discourse analysis. In J. C. a. P. Trudgill (Ed.), The Sociolinguistics Reader. Volume 2: Gender and Discourse (pp. 367-393). London: Arnold.

Verderber, K. S., Verderber, R. F., and Berryman-Fink, C. (2009). Inter-Act: Interpersonal Communication Concepts, Skills, and Contexts (12 ed.). USA: Oxford University Press.

Vine, B. (2004). Getting Things Done at Work: The Discourse of Power in Workplace Interaction. Amsterdam/Philadelphia: John Benjamins.

Vine, B. (2009). Directives at work: Exploring the contextual complexity of workplace directives Journal of Pragmatics, 36(5), 945-964.

Vine, B., Holmes, J., Marra, M., Pfeifer, D., and Jackson, B. (2008). Exploring coleadership talk through interactional sociolinguistics. Leadership, 4(3), 339-360.

Vine, B., Kell, S., Marra, M., and Holmes, J. (2009). Boundary-marking humour. Institutional, gender and ethnic demarcation in the workplace In N. R. Norrick and D. Chiaro (Eds.), Humour in Interaction (pp. 125-141). Amsterdam/Philadelphia: John Benjamins.

Wadsworth, Y. (1998). What is Participatory Action Research? . Retrieved 10/1/2010. URL: http://www.scu.edu.au/schools/gcm/ar/ari/p-ywadsworth98.html

Wagner, J. (1995). Negotiating activity in technical problem solving. In A. Firth (Ed.), The Discourse of Negotiation: Studies of Language in the Workplace (pp. 113-135). Oxford: Pergamon.

Waldvogel, J. (2005). The Role, Status and Style of Workplace Email: A Study of Two New Zealand Workplaces. Unpublished $\mathrm{PhD}$, Victoria University, Wellington.

Watson, B. M., and Gallois, C. (1999). Communication accommodation between patients and health professionals: themes and strategies in satisfying and unsatisfying encounters. International Journal of Applied Linguistics, 9(2), 167180.

Weatherall, A., and Stubbe, M. (2009). Affiliation During Complaint Calls in Institutional Talk. Paper presented at the Panel on 'Affectivity in institutional talk', (Convenor Elisabeth Couper-Kuhlen). International Pragmatics Association Conference (IPRA) 13- 17 July 2009, Melbourne, Australia.

Weatherall, A., Stubbe, M., Sunderland, J., and Baxter, J. (In press). Conversation analysis and critical discourse analysis in language and gender research: approaches in dialogue. In J. Holmes and M. Marra (Eds.), Femininity, Feminism and Gendered Discourse. Newcastle-upon-Tyne: Cambridge Scholars Publishing.

Weedon, C. (1987). Feminist Practice and Poststructuralist Theory. Oxford: Basil Blackwell.

Weeks, M. (2005). Nurse-physician communication in the perioperative environment: discourse and actions to transform health care. Canadian Operating Room Journal, 23(1), 51-54.

Weigand, E. (1999). Misunderstanding: The standard case. Journal of Pragmatics, 31, 763-785. 
Weinger, M. B., Pantiskas, C., Wiklund, M. E., and Carstensen, P. (1998). Incorporating human factors into the design of medical devices. JAMA, 280(17), 1484.

Weiss, G., and Wodak, R. (Eds.). (2003). Critical Discourse Analysis: Theory and Interdisciplinarity. Basingstoke: Palgrave Macmillan.

Wenger, E. (1998). Communities of Practice: Learning, Meaning and Identity. Cambridge: Cambridge University Press.

Wenger, E. C., and Snyder, W. M. (2000). Communities of practice: The organizational frontier. Harvard Business Review, Jan-Feb 2000, 139-145.

West, C. (1984). Routine Complications: Troubles with Talk between Doctors and Patients. Bloomington: Indiana University Press.

West, C., and Frankel, R. (1991). Miscommunication in medicine. In N. Coupland, H. Giles and J. M. Wiemann (Eds.), "Miscommunication" and problematic talk (pp. 166-194). US: Sage Publications, Inc Thousand Oaks.

Wetherell, M. (1998). Positioning and interpretive repertoires: conversation analysis and post-structuralism in dialogue. Discourse and Society, 9(3), 387412.

Wetherell, M., and Potter, J. (1992). Mapping the Language of Racism: Discourse and the Legitimation of Exploitation. Brighton: Harvester/Wheatsheaf.

Wetherell, M., Stiven, H., and Potter, J. (1987). Unequal egalitarianism: A preliminary study of discourses concerning gender and employment opportunities. British Journal of Social Psychology, 26(1), 59-71.

Wheelan, S. A., and Williams, T. (2003). Mapping dynamic interaction patterns in work groups. Small Group Research, 34(4), 443-467.

Wilkinson, S., and Kitzinger, C. (2006). Surprise as an interactional achievement: reaction tokens in conversation. Social Psychology Quarterly, 69(2), 150-182.

Williams, A. (1999). Communication Accommodation Theory and miscommunication: issues of awareness and communication dilemmas. International Journal of Applied Linguistics, 9(2), 151-165.

Williams, H. (Ed.) (1971). Wellington: Legislation Direct.

Willing, K. (1992). Talking it Through. Clarification and Problem-solving in Professional Work. Sydney: Macquarie University.

Willing, K. (1997). Modality in task-oriented discourse: The role of subjectivity in 'getting the job done'. Prospect, 12(2), 33-42.

Wilson, E. (2000). Inclusion, exclusion and ambiguity: the role of organisational culture. Personnel Review, 29(3), 274-303.

Wodak, R. (1996). The Disorders of Discourse. London: Longman.

Woods, D. D., Patterson, E. S., and Cook, R. I. (2007). Behind human error: Taming complexity to improve patient safety. In P. Carayon (Ed.), Handbook of Human Factors and Ergonomics in Health Care and Patient Safety (pp. 459476). Mahwah, New Jersey: Lawrence Erlbaum Associates.

Yeatman, A. (1994). Feminism and power. Women's Studies Journal, 10, 79-100. 
